\title{
Strategy-proof voting schemes on Euclidean spaces
}

Citation for published version (APA):

van der Stel, J. (1993). Strategy-proof voting schemes on Euclidean spaces. [Doctoral Thesis, Maastricht University]. Datawyse / Universitaire Pers Maastricht. https://doi.org/10.26481/dis.19930610js

Document status and date:

Published: 01/01/1993

DOI:

10.26481/dis.19930610js

Document Version:

Publisher's PDF, also known as Version of record

\section{Please check the document version of this publication:}

- A submitted manuscript is the version of the article upon submission and before peer-review. There can be important differences between the submitted version and the official published version of record.

People interested in the research are advised to contact the author for the final version of the publication, or visit the DOI to the publisher's website.

- The final author version and the galley proof are versions of the publication after peer review.

- The final published version features the final layout of the paper including the volume, issue and page numbers.

Link to publication

\footnotetext{
General rights rights.

- You may freely distribute the URL identifying the publication in the public portal. please follow below link for the End User Agreement:

www.umlib.nl/taverne-license

Take down policy

If you believe that this document breaches copyright please contact us at:

repository@maastrichtuniversity.nl

providing details and we will investigate your claim.
}

Copyright and moral rights for the publications made accessible in the public portal are retained by the authors and/or other copyright owners and it is a condition of accessing publications that users recognise and abide by the legal requirements associated with these

- Users may download and print one copy of any publication from the public portal for the purpose of private study or research.

- You may not further distribute the material or use it for any profit-making activity or commercial gain

If the publication is distributed under the terms of Article $25 \mathrm{fa}$ of the Dutch Copyright Act, indicated by the "Taverne" license above, 
Strategy-Proof

Voting Schemes on

Euclidean Spaces 


\section{Strategy-Proof}

\section{Voting Schemes on}

\section{Euclidean Spaces}

\section{PROEFSCHRIFT}

ter verkrijging van de graad van doctor aan de Rijksuniversiteit Limburg te Maastricht, op gezag van de Rector Magnificus, Prof.Mr. M.J. Cohen, volgens het besluit van het College van Dekanen, in het openbaar te verdedigen op donderdag 10 juni 1993 om 16.00 uur

door

Johannes van der Stel

geboren te Goes in 1960

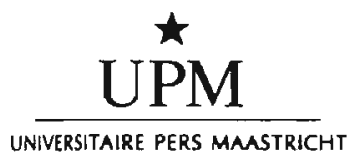


Promotores : Prof.dr.ir.drs. O.J. Vrieze

: Prof.dr. H.J.M. Peters

Co-promotor: Dr. A.J.A. Storcken

Leden van de beoordelingscommissie:

Prof.dr. S.H. Tijs (voorzitter)

Prof.dr.ir. A.W.J. Kolen

Prof.dr. H.Th. Jongen (RWTH Aachen)

Prof.dr. P.H.M. Ruys (KU Brabant)

Datawyse | Universitaire Pers Maastricht

ISBN 90-5278-083-8 


\section{Acknowledgements}

Most of the research described in this dissertation is based on joint work with Hans Peters and Ton Storcken. Without their efforts it would have been impossible for me to write this thesis. Their expertise on the fields of social choice theory and mathematical economics was of great value to me.

I am grateful to Koos Vrieze. It was very pleasant to work for him. His guidance led to an ideal research environment.

I wish to thank all my colleagues in this environment, and also the people of the department of Computer Science. In particular I mention Jeroen Kuipers, Frits Spieksma, Maarten Oosten, Jacques Lenting and Rossina Slavova.

Further I want to express my gratitude to Martina Roelse. The telephone conversations we had were a source of inspiration to me, not only from a mathematical point of view.

I thank all people who have been of great mental support to me. I mention my parents, my grandparents, my aunt, Cor and Suus.

I am very grateful to Marlies Haenen for typing out this manuscript. Her support was a decisive factor in the accomplishment of this monograph. I wish to thank Marijke Verheij, who also played an important role in this process. Finally, I express my gratitude to René Druif, who designed the front page. 


\section{Contents}

1 Introduction and Preliminaries 1

1.1 Introduction . . . . . . . . . . . . . . . . . 1

1.1.1 Subject of the thesis . . . . . . . . . . . 1

1.1 .2 History and related literature . . . . . . . . . 2

1.1 .3 Outline . . . . . . . . . . . . . . . 5

1.2 Model, definitions, preliminaries . . . . . . . . . . . 7

1.3 Voting schemes for location problems . . . . . . . . 16

1.3.1 The mean prescheme . . . . . . . . . . . . 17

1.3.2 The median prescheme . . . . . . . . . . . . 17

1.3.3 Coordinatewise median schemes . . . . . . . . 17

1.3.4 Coordinatewise vetopreschemes . . . . . . . . . 20

2 Pareto Optimality and Strictly Convex Norms 23

2.1 Introduction . . . . . . . . . . . . . . . 23

2.2 Norms, strict convexity and orthogonality . . . . . . . 25

2.3 Pareto optimality and strategy-proofness . . . . . . . . . 41

2.4 Two person location problems in normed spaces .... 58

2.5 Three person location problems in $\mathbf{R}^{m} \ldots \ldots \ldots \ldots$

2.6 Three person location problems in $\mathbf{R}^{2} \ldots \ldots \ldots 69$

$2.7 n$-person location problems in $\mathbf{R}^{2} \ldots \ldots \ldots \ldots$

2.8 Coalitional strategy-proofness $\ldots \ldots \ldots \ldots$

2.9 Conclusions and cren problems . . . . . . . . . . . . . 88

3 Pareto Optimality and Inner Product Metrics 91

3.1 Introduction . . . . . . . . . . . . . . . . . . . 91

3.2 Location problems and inner products . . . . . . . . . . 92

3.3 Pareto optimality and strategy-proofness in $\mathbf{R}^{m} \ldots \ldots$. . . 97

3.4 Further main results . . . . . . . . . . . . . . . . . 102

3.5 Conclusion . . . . . . . . . . . . . . 106 
4 Range Convexity and Euclidean Norms 107

4.1 Introduction . . . . . . . . . . . . . . . . . . . 107

4.2 One person Euclidean location problems on $\mathbb{R}^{m} \ldots \ldots \ldots 109$

4.3 Two person Euclidean location problems . . . . . . . . . 111

4.4 Two dimensional Euclidean location problems . . . . . . 116

4.5 Conclusion . . . . . . . . . . . . . . . . . . 120

5 Uncompromisingness 121

5.1 Introduction . . . . . . . . . . . . . . 121

5.2 The class of preferences $P^{*}$ and uncompromisingness . . . 123

5.3 Coordinatewise veto schemes . . . . . . . . . . 124

5.4 Characterizing coordinatewise veto schemes . . . . . . . 131

5.5 Special cases . . . . . . . . . . . . . 135

5.5.1 The subclasses $p^{1}$ and $p^{\infty} \ldots \ldots \ldots \ldots 135$

5.5 .2 Separable and quadratic preferences . . . . . . 141

5.6 Conclusion . . . . . . . . . . . . . . . 145

6 Independence of Irrelevant Alternatives 147

6.1 Introduction . . . . . . . . . . . . . . 147

6.2 Preliminaries . . . . . . . . . . . . . . . . . 148

6.3 IIA and strategy-proofness . . . . . . . . . . . . . 149

6.4 IIA and Euclidean preferences . . . . . . . . . . 151

6.5 Conclusion . . . . . . . . . . . . . . . . 154

$\begin{array}{ll}\text { Appendix A } & 155\end{array}$

$\begin{array}{ll}\text { References } & 159\end{array}$

List of Symbols $\quad 165$

$\begin{array}{ll}\text { Subject Index } & 167\end{array}$

Author Index 171

$\begin{array}{ll}\text { Samenvatting } & 173\end{array}$ 


\section{Chapter 1}

\section{Introduction and Preliminaries}

\subsection{Introduction}

\subsubsection{Subject of the thesis}

In many problems of collective choice, a group of individuals has to reach an agreement on the choice of one alternative from a given set of alternatives. A simple way to reach such an agreement is presented by a voting procedure of the following kind. Each individual mentions one alternative. Then a rule is invoked which selects a unique compromise alternative. It is important that this rule is fixed apriori, i.e., before the actual voting. Such a voting procedure will be called a voting scheme (or shortly: a scheme).

In their voting behaviour, the individuals are guided by their preference over the set of alternatives. Throughout we assume that these preferences have best points. Concerning the alternatives we assume that these can be represented by points in $m$-dimensional Euclidean space. The set of alternatives we consider will usually be a continuum. The resulting decision problem is called an $m$-dimensional $n$ person location problem.

What is termed here a location problem, can be a location problem in the literal sense of the word, e.g., concerning the location of a gas station along a road $(m=1)$, or the location of a public facility - a library, a swimming pool - in the plane ( $m=2)$, or a facility in a high building ( $m=3)$. But numerous other interpretations are possible:

- voting, with candidates on a scale from left to right $(m=1)$. 
- investment problems: a board of directors must assign money to $m$ different projects.

- multi criteria decision making: a group of policy makers has to attach weights to $m$ criteria.

- prediction problems: $n$ economists attach probabilities to $m$ possible states of the economy, and a compromise prediction must be reached.

- more abstract problems in which a group of $n$ people has to assign values to a set of $m$ decision variables.

We consider the individual preferences as private information. Given a voting scheme, this implies that individuals may have an incentive to misrepresent their preferences strategically, in order to obtain a better social outcome. In this monograph, we concentrate on strategy-proof voting schemes; if such a voting scheme is used no individual can gain by misrepresenting his preference. Strategy-proofness is a very desirable property for voting schemes because it stimulates individuals to mention their best points. This has some important advantages:

- In order to obtain a 'good' outcome, it seems to be inevitable to obtain reliable information concerning the individual preferences.

- No individual needs to be occupied with strategic behaviour in the voting process. It suffices to mention a best alternative. He cannot gain by deviating from it .

- Individuals who mention a best point are not put at a disadvantage with respect to individuals who deviate (if they were, that would be a reason for deviating themselves).

We will be mainly concerned with the search for strategy-proof voting schemes. Some other properties which one might consider reasonable for voting schemes are described in section 1.2 .

Our approach is axiomatic: given certain properties, we try to characterize the class of all voting schemes which have these properties. These characterizations can be considered as the main results of the monograph.

\subsubsection{History and related literature}

After Arrow's (1963) impossibility theorem, Gibbard (1973) and Satterthwaite (1975) established the following negative result: If there are at least 
three alternatives, then the only strategy-proof and surjective social choice rules are the dictatorial rules. Satterthwaite (1975) also showed that under Pareto optimality and in the classical discrete framework of social choice theory with all linear or weak orders allowed, the strategy-proofness condition is logically equivalent to Arrow's (1963) independence of irrelevant alternatives condition, and therefore impossibility results were to be expected.

The issue of strategy-proofness was brought to new life by the work of Laffond (1980) and Moulin (1980). The assumptions these authors made were strongly related to the single-peakedness condition, introduced earlier by Black (1948, see also Black, 1958). They consider continuum domains of alternatives - for instance, the real line in Moulin (1980) - and restricted domains of preferences. Furthermore, they studied voting schemes as described in subsection 1.1.1. In these works, as well as in many subsequent papers, nonconstant nondictatorial strategy-proof voting schemes were characterized in various contexts. Also, this approach made the theory of social choice more readily applicable to standard micro-economic or political models.

In Moulin (1980) the set of alternatives is the whole real line. Each individual reports a point; only single-peaked preferences are allowed, i.e., preferences with a unique best element and decreasing when moving away from this element in either of the two directions. Moulin considers three conditions on voting schemes: strategy-proofness, anonymity, and continuity. He shows that any scheme satisfying the first two conditions must be of the following form. Choose $n+1$ points $a_{1}, a_{2}, \ldots, a_{n+1}$ in $\mathbb{R} \cup\{+\infty,-\infty\}$. If the $n$ individuals report the points $x_{1}, x_{2}, \ldots, x_{n}$, then the scheme assigns the median point of the $2 n+1$ points $a_{1}, \ldots, a_{n+1}, x_{1}, \ldots, x_{n}$ - that is, the middle point after all points arranged in nondecreasing order. From this, it follows that if Pareto optimality is added, the class of schemes satisfying the three properties is the subclass of schemes determined by $n-1$ points instead of $n+1$. Proposition 4 in Border and Jordan (1983) implies that both results still hold if only Euclidean preferences are considered. In a Euclidean preference, one point is preferred to another if it is closer to the best point, measured according to Euclidean distance. So a Euclidean preference is completely characterized by its best point. Kim and Roush (1981) provide an alternative axiomatic characterization of the same class of schemes.

Laffond (1980) gives a detailed analysis of the case of two agents with Euclidean or quadratic preferences on higher dimensional Euclidean space. He characterizes the class of anonymous, strategy-proof, surjective schemes by means of so-called option sets (Barberà and Peleg, 1990), which describe 
the points attainable by one agent if the preference(s) of the other agent(s) are being held fixed, for a given voting scheme.

Kim and Roush (1984) also extend Moulin's results to higher dimensions. Kim and Roush characterize all componentwise continuous, anonymous, and strategy-proof schemes with the Euclidean plane as set of alternatives and only Euclidean preferences allowed. These schemes must be coordinatewise median schemes with respect to an orthogonal basis in the Euclidean plane with $n+1$ constant points. This result is used in chapters 3 and 4 , where we show that componentwise continuity can be replaced by other properties. By adding Pareto optimality to the other three conditions, Kim and Roush show that only coordinate median schemes without constant points suffice. Because of the definition of a median, this implies that the number of agents must be odd. In chapter 3 the same class of schemes is characterized without the continuity condition.

Another closely related paper is Border and Jordan (1983), already mentioned above. In their main result, these authors characterize the class of all voting schemes that are unanimous and strategy-proof with respect to the class of separable quadratic preferences. Therefore, their strategy-proofness condition (which they call "straightforwardness") is stronger than the one imposed by Kim and Roush (1984), or the one used in chapters 2, 3 and 4 in this monograph. In particular, the coordinate system with respect to which their preferences are defined determines the coordinate system with respect to which the characterized voting schemes are defined whereas, in the other mentioned research, every voting scheme determines its own orthogonal basis by which it can be described. Chichilnisky and Heal (1981) adopt the same framework as Border and Jordan (1983), but replace the condition of unanimity of a scheme by the requirement that its range be rectangular. In chapter 5 of this thesis the work of Border and Jordan is generalized to other domains of alternatives and other domains of preferences.

Still other work in the same area was done by Bordes et al. (1989), who, in the larger part of their paper, concentrate on the (stronger) coalitional strategy-proofness condition, and (not surprisingly) on impossibility results. Further, we mention Le Breton and Sen (1991), Barberà et al. (1990), Barberà et al. (1991), and Barberà et al. (1992). In a wider context, we mention the work of Barberà and Peleg (1990), Moreno and Walker (1989), Moreno (1991), and Zhou (1991).

Since, in the classical context, strategy-proofness is closely related to Arrow's independence of irrelevant alternatives (IIA) condition, the question arises how these conditions are related in models with a continuum of alter- 
natives. It turns out that in this context IIA - which concerns the orderings of all alternatives - is much stronger than strategy-proofness - which can be formulated as was done above with respect to the best elements of the individual preferences only. For details we refer to Bordes and Le Breton (1989) and chapter 6. Moulin (1984) considers the combination of Nash's (1950) and Arrow's IIA for one-dimensional voting schemes.

Another strand of literature, not concerned with strategy-proofness but with notions of fairness, studies location problems by cooperative gametheoretical methods. (In contrast, strategy-proofness is typically a noncooperative game-theoretical concept.) In Rosenmüller (1982), location problems are related to so-called nonsidepayment games, which, however, must be interpreted in a different way. Coalitions are not in a position to enforce their worths but, rather, these worths represent what would be ideal given that outside players were not present. It is assumed that information is not private; players cannot lie. Fair schemes can be obtained by cooperative gametheoretical methods. A similar line of thought is pursued in Ostmann (1982), Ostmann and Straub (1979), Richter (1979), and Rosenmüller (1980).

\subsubsection{Outline}

Section 1.2 gives a formal description of the model. Definitions and notations which will be used throughout the monograph are introduced here. In section 1.3 we discuss some examples of voting schemes. In chapter 2 to 4 we focus on classes of preferences which are defined by a metric on the set of alternatives, in particular metrics which are induced by a strictly convex norm. Before we can devote ourselves to the study of resulting location problems, we need to review and develop some theory on norms. This is done in section 2.2 . We study strict convexity with respect to norms, and some orthogonality concepts which are adequate for all arbitrary norms. In section 2.3 some scheme properties are reformulated with respect to norms. Next we study implications of Pareto optimality and strategy-proofness with respect to strictly convex norms. One of the most important results of this section is proposition 2.1 , which states that with respect to a strictly convex norm, several Pareto optimality concepts (as defined in section 1.2) are equivalent. This leads to a geometrical characterization of Pareto optimality with respect to such a norm for the two-dimensional case (see theorem 2.1). Section 2.4 deals with two person location problems. One of the conclusions is, that all schemes which satisfy Pareto optimality and strategy-proofness with respect to a strictly convex norm must be dictatorial (given that the set of 
alternatives is open). Using these results, we obtain our first main result: If the number of agents is even, then there are no voting schemes which are anonymous, and Pareto optimal and strategy-proof with respect to strictly convex norms.

Sections 2.5 and 2.6 deal with the three person location problem, where the set of alternatives coincides with the whole $m$-dimensional Euclidean space. In section 2.5 we study $m$-dimensional location problems with $m>$ 1. We show that strategy-proofness and Pareto optimality with respect to a strictly convex norm imply componentwise continuity. This enables us to obtain our second main result: A characterization of all schemes which satisfy anonymity, and Pareto optimality and strategy-proofness with respect to a strictly convex norm.

The last mentioned result is generalized in section 2.7 for an arbitrary odd number of agents by theorem 2.5: this class of schemes coincides with the class of coordinate median schemes with respect to an orthogonal (in the sense of the chosen norm) basis without constant points.

In section 2.8 it is demonstrated that Pareto optimality and coalitional strategy-proofness (a stronger version of strategy-proofness) leads to an impossibility result, i.e., a dictatorial scheme.

In chapter 3 we focus on norms which are induced by an inner product. All these norms are strictly convex, and closely related to the Euclidean metric. The set of alternatives coincides with $m$-dimensional Euclidean space, with $m \geq 2$. Using a result of Kim and Roush (see subsection 1.1.2) we can find a shortcut for the proof of theorem 2.4 for this kind of metric.

Furthermore, we obtain the additional result that in the $m$-dimensional case with $m \geq 3$, no voting schemes exist which satisfy anonymity, and strategy-proofness and Pareto optimality with respect to the Euclidean metric. This result is not valid for arbitrary strictly convex norms.

In chapter 4 the set of alternatives is again $m$-dimensional Euclidean space, with $m \geq 2$. We study schemes which satisfy strategy-proofness with respect to the Euclidean metric, and range convexity. Section 4.1 deals with the one person case, where the equivalence of range convexity and continuity is demonstrated under the assumption of strategy-proofness. The two person case is studied in section 4.3. The results of this section are used in section 4.4, where we handle the two-dimensional $n$ person location problem. The first main result in this chapter is that in the result of Kim and Roush (1984) continuity can be replaced by range convexity.

Since surjectivity (unanimity) implies range convexity, it is now straightforward to characterize all schemes which satisfy anonymity, strategy-proof- 
ness and surjectivity (unanimity): this result is our second main result in chapter 4 .

In chapter 5 we consider a much broader class of preferences, denoted by $p^{*}$. Under the assumption that the set of alternatives is an open subset of $m$ dimensional Euclidean space with $m \geq 2$, we prove that the class of strategyproof (with respect to $P^{*}$ ) schemes coincides with the class of coordinatewise veto schemes with respect to the standard basis. In subsection 5.5 .2 it is proved that strategy-proofness with respect to a subclass of $P^{*}$ (the class of separable quadratic preferences, also studied by Border and Jordan, 1983) suffices to characterize this class of voting schemes. In subsection 5.5.1 it is shown that the same result remains valid for another restricted class of preferences and other domains of alternatives (for instance a grid).

In chapter 6 we study implications of Arrow's independence of irrelevant alternatives conditions in location problems. It is shown that this condition is much more restrictive than strategy-proofness, and usually leads to an impossibility result, i.e., constant or dictatorial scheme, even if we restrict attention to Euclidean preferences.

For further discussion on related literature, we refer to the final sections of the chapters.

\subsection{Model, definitions, preliminaries}

Let $m \in \mathbb{N}^{1}$, and denote by $M:=\{1,2, \ldots, m\}$ the set of coordinates. A nonempty subset $A$ of $\mathbb{R}^{m}$ is called a set of alternatives. $N:=\{1,2, \ldots, n\}$, where $n \in \mathbb{N}$, is the set of individuals or agents (or voters).

A voting scheme (or shortly: a scheme) is a map $\varphi: A^{N} \rightarrow A$. The interpretation is that each individual $i$ reports a point $p(i) \in A$. A scheme $\varphi$ assigns to each profile $p=(p(1), \ldots, p(n))$ a point $\varphi(p)$ in $A . \varphi(p)$ is also called the compromise point. A map $\varphi: A^{N} \rightarrow \mathbb{R}^{m}$ is called a prescheme.

In reporting a point from $A$, an individual will be guided by his preference over $A$. In this thesis a preference on $A$ is a binary relation $R$ on $A$ such that:

(i) For all $a, b \in A:(a, b) \in R$ or $(b, a) \in R$ (completeness),

(ii) There is a $b \in A$ with $(b, a) \in R$ for all $a \in R$.

Such a preference can be interpreted as follows. Let $a, b \in A$. Then $(a, b) \in R$ if and only if $a$ is weakly preferred to $b$ according to $R$. So we have:

$\mathbb{N}:=\{1,2,3, \ldots\}$. 


$$
\begin{aligned}
& \text { if }(a, b) \in R \text { and }(b, a) \in R \text { then } a \text { and } b \text { are valued equally, } \\
& \text { if }(a, b) \in R \text { and }(b, a) \notin R \text { then } a \text { is considered to be better than } b \text {. }
\end{aligned}
$$

The conditions (i)-(ii) mean the following: (i) expresses that every pair of alternatives can be compared, and (ii) states that there is at least one best alternative. The set of best elements of $R$ is defined by

$$
\operatorname{best}(R):=\{x \in A: \forall y \in A[(x, y) \in R]\},
$$

the subset of elements of $A$ that are (weakly) preferred to all other elements of $A$. An element of best $(R)$ is called a best point, bliss point or ideal point. As each profile $p$ consists of a sequence of vectors, we will sometimes denote $p$ in terms of these vectors. Let $S_{1}, S_{2}, \ldots S_{k} \subset N$ be pairwise disjoint with $\cup_{j=1}^{k} S_{j}=N$. Let $x(1), x(2), \ldots, x(k) \in A$. Then $\left\langle x(1)^{S_{1}}, \ldots, x(k)^{S_{k}}\right\rangle$ denotes the profile $p$ such that $p(j)=x(i)$ for all $j \in S_{i}$. So for instance, $\left\langle x^{N}\right\rangle$ denotes the profile $p$ with $p(i)=x$ for all $i \in N$ and $x \in A$. For all $m \times m$ matrices $V$ and profiles $p, q \in\left(\mathbb{R}^{m}\right)^{N}, q=V p$ means that $q(j)=V p(j)$ for $j=1, \ldots, n$. For $p \in\left(\mathbb{R}^{m}\right)^{N}$ and $S \subset N,\left.p\right|_{S}$ denotes the restriction of $p$ to $S$. So for instance, if $\left.p\right|_{S}=\left.q\right|_{s}$, then $p(j)=q(j)$ for all $j \in S$. Furthermore, for all $p, q \in A^{N}, S \subset N$ the notation $p=s q$ will mean that $\left.p\right|_{S}=\left.q\right|_{S}$.

The main subject of this thesis is the search for schemes which satisfy certain properties. Before we discuss these properties, we define two special voting schemes:

(i) The constant scheme. This voting scheme is defined by $\varphi(p)=c$ for all $p \in A^{N}$, where $c \in A$ is given. So this scheme chooses always the same alternative, no matter what the reported opinions might be.

(ii) The dictatorial scheme. For this voting scheme there is an $i \in N$ given such that $\varphi(p)=p(i)$ for all $p \in A^{N}$. Agent $i$, called the dictator, determines where the compromise point will be. The opinions of the other agents are not taken into account.

Both voting schemes are regarded to be very unattractive. However, in social choice theory, there are many cases where only these voting schemes satisfy a certain combination of properties. If this is the case, one considers this as an impossibility result.

We now describe some properties one might impose upon a scheme, which all have to do with the positioning of the compromise point in the alternative set, in relationship to the positioning of the individually reported points. Let $P$ be a class of preferences on $A$. Let $\varphi$ be a (pre)scheme. Then: 
(i) PO: $\varphi$ is called Pareto optimal with respect to $P$, if there are no $x \in A$, $R(i) \in P(i \in N)$, and $p \in A^{N}$ such that:
(a) $p(i) \in \operatorname{best}(R(i))$ for all $i \in N$,
(b) $(x, \varphi(p)) \in R(i)$ for all $i \in N$,
(c) $(\varphi(p), x) \notin R(i)$ for some $i \in N$.

(ii) SPO: $\varphi$ is called strongly Pareto optimal with respect to $P$, if there are no $x \in A, R(i) \in P(i \in N)$, and $p \in A^{N}$ such that:
(a) $p(i) \in \operatorname{best}(R(i))$ for all $i \in N$,
(b) $(x, \varphi(p)) \in R(i)$ for all $i \in N$,
(c) $x \neq \varphi(p)$.

(iii) WPO: $\varphi$ is called weakly Pareto optimal with respect to $P$, if there are no $x \in A, R(i) \in P$, and $p \in A^{N}$ such that for all $i \in N$ :
(a) $p(i) \in \operatorname{best}(R(i))$,
(b) $(\varphi(p), x) \notin R(i)$.

(iv) UN: $\varphi$ is called unanimous if for all $x \in A: \varphi\left(\left\langle x^{N}\right\rangle\right)=x$.

(v) SURJ: $\varphi$ is called surjective if $\varphi\left(A^{N}\right)^{2}=A$.

(vi) $\mathrm{RC}: \varphi$ is called range convex, if $\varphi\left(A^{N}\right)$ is a convex set.

Pareto optimality means that no agent can gain without some other agent losing, in terms of the preferences of which the reported points are ideal points. Strong Pareto optimality means that there is no other alternative which is for every agent at least as good as the compromise point. Weak Pareto optimality means that there is no alternative which is better than the compromise point for every agent.

It is clear that SPO implies PO, and that PO implies WPO. SPO seems to be an unreasonably strong requirement. However, it will turn out in chapter 2, that with respect to some classes of preferences, PO, SPO and WPO are equivalent (cf. proposition 2.1).

There is some ambiguity in literature about the different Pareto optimality concepts. Muller and Satterthwaite (1985) for instance speak of the strong Pareto criterion, whenever they mean the property we called PO.

${ }^{2}$ If $Y$ and $Z$ are sets $f: Y \rightarrow Z$ and $X \subset Y$, then $f(X):=\{f(x): x \in X\}$. 
Another term which is used sometimes instead of Pareto optimality, is 'effciency' (cf. Kim and Roush, 1984).

Unanimity, surjectivity and range convexity are defined without reference to some class of preferences. Unanimity means that if all agents report the same point, the compromise point will be this point. Surjectivity means that all alternatives may be chosen, i.e., for each alternative there exists a profile such that this alternative is the compromise point for this profile. Surjectivity is often named 'citizen's sovereignty' in the social choice literature. There is also another way to look at surjectivity: if a voting scheme is surjective, the set of alternatives is apparantly such that no agent has the opportunity to choose an alternative which can never become the compromise point. It is obvious that unanimity implies surjectivity. There are many classes of preferences, however, with respect to which strategy-proofness implies the equivalence of these two properties (see corollary 1.1). Under mild conditions unanimity in turn is implied by weak Pareto optimality (see lemma 1.3).

Range convexity means that the range of a voting scheme is convex. So, if $A$ is convex, surjectivity implies range convexity.

Summarizing, under mild conditions we have: $\mathrm{SPO} \Rightarrow \mathrm{PO} \Rightarrow \mathrm{WPO} \Rightarrow$ $\mathrm{UN} \Rightarrow \mathrm{SURJ} \Rightarrow \mathrm{RC}$. The constant scheme satisfies RC. If there are at least two alternatives, it does not satisfy SURJ nor UN. With respect to most of the preference classes we will study in this thesis, it does not satisfy the Pareto optimality conditions. By definition, the dictatorial scheme satisfies WPO, UN, and SURJ. In many cases it also satisfies RC, SPO and PO.

We now discuss some different versions of nonmanipulability. Let $P$ be a class of preferences on $A$. Let $\varphi$ be a (pre)scheme. Then:

(i) SP: $\varphi$ is called strategy-proof with respect to $P$, if for every preference $R \in P$, every $i \in N$ and all $p, q \in A^{N}$ with $p(i) \in \operatorname{best}(R)$ and $p=N \backslash\{i\} q$, we have: $(\varphi(p), \varphi(q)) \in R$.

(ii) ISP: $\varphi$ is called intermediate strategy-proof with respect to $P$, if for every preference $R \in \mathcal{P}$, every $S \subset N$ and all $p, q \in A^{N}$ with $p(S) \subset$ best $(R)$ and $p={ }_{N \backslash S} q$, we have: $(\varphi(p), \varphi(q)) \in R$.

(iii) CSP: $\varphi$ is called coalitionally strategy-proof with respect to $P$, if for all $S \subset N, R(i) \in P(i \in S), p, q \in A^{N}$ with

(a) $p(i) \in \operatorname{best}(R(i))$ for all $i \in S$,

(b) $p={ }_{N \backslash S} q$, 
(c) $(\varphi(q), \varphi(p)) \in R(i)$ for all $i \in S$,

we have that $(\varphi(p), \varphi(q)) \in R(i)$ for some $i \in S$.

(iv) SSP: $\varphi$ is called strictly strategy-proof with respect to $P$, if for every preference $R \in P$, every $i \in N$ and all $p, q \in A^{N}$ with $p(i) \in \operatorname{best}(R)$ and $p={ }_{N \backslash\{i\}} q$, we have either $(\varphi(q), \varphi(p)) \notin R$ or $\varphi(p)=\varphi(q)$.

A scheme is strategy-proof with respect to $P$, if for each agent it is always (i.e. independent of the other agents) optimal to report a best element of his true preference. Intermediate strategy-proofness with respect to $P$ states that if all agents belonging to any subset $S \subset N$ have the same preference, they do not gain by simultaneously reporting different points. Coalitional strategy-proofness with respect to $\mathcal{P}$ requires that there is no nonempty subset $S \subset N$ such that all agents in this subset can gain by simultaneously reporting different points.

Note that CSP implies ISP, and ISP implies SP. Observe further that these properties only make sense if there are $R \in P$ such that best $(R)$ is nonempty, otherwise every scheme satisfies these properties. The same is true for PO.

Strict strategy-proofness implies that if an individual $i$ deviates from a best element of his true preference, the old and new compromise point should be equal if he is indifferent about them. In other words: if an agent succeeds in changing the compromise by misrepresenting his true preference, he will be punished for this. Also SSP implies SP. Since no one can influence the compromise point when a constant scheme is used, this scheme satisfies SP, ISP, CSP and SSP.

When a dictatorial scheme is used, only the dictator can influence the compromise point. Since this compromise point always coincides with the point he reports, he would only punish himself by not choosing one of his bliss points. So also the dictatorial scheme always satisfies SP, ISP and CSP. When each preference has a unique bliss point, the dictatorial scheme also satisfies SSP.

The next two properties has to do with the distribution of power over the agents. Let $\varphi$ be a (pre)scheme. Then:

(i) AN: $\varphi$ is called anonymous if $\varphi(p \circ \sigma)=\varphi(p)$ for every $p \in A^{N}$ and every permutation $\sigma: N \rightarrow N$.

(ii) ND: $\varphi$ is called nondictatorial if $\varphi$ is not a dictatorial scheme. 
Anonymity states that interchanging agents does not influence the compromise point. In general $\mathrm{AN}$ is far more restrictive than ND. Note further that both properties are defined without reference to some class of preferences.

The constant scheme always satisfies AN and ND (except for the case in which there is only one alternative). Except for trivial cases, the dictatorial scheme is not anonymous. By definition, it does not satisfy ND.

In the following example we describe another voting scheme, known as majority rule.

Example 1.1 A church council has 11 members. It has to decide on the cutting of an elm on the churchyard. There are two alternatives:

0 : the elm will be cut,

1: the elm will not be cut.

The voting scheme they use is majority rule. In the case of two alternatives this scheme is such that the alternative which is chosen by a majority will be elected. Formally, we can describe this situation as follows. $n=11, m=1$, $A=\{0,1\}$. The voting scheme $\varphi: A^{N} \rightarrow A$ is such that we have for all $p \in A^{N}: 3$

$$
\begin{aligned}
\varphi(p) & =0 \text { if }|\{i \in N: p(i)=0\}| \geq 6 \\
& =1 \text { otherwise. }
\end{aligned}
$$

$\varphi$ satisfies WPO. PO and SPO are satisfied if no member of the council is indifferent between 0 and 1 . The unanimity and surjectivity of $\varphi$ are obvious. By reporting his bliss point, an agent enlarges the chance that this alternative will be chosen. So no one has an incentive to deviate from his bliss point. Therefore, $\varphi$ satisfies SP, CSP and ISP. As long as no agent is indifferent about the alternatives, $\varphi$ also satisfies SSP. Since the majority rule is impartial ('one man, one vote'), $\varphi$ is anonymous. The nondictatoriness of $\varphi$ is obvious.

This example shows that majority rule is very attractive if there are only two alternatives. If there are more alternatives, majority rule turns out to be manipulable (for a clear explanation on this subject, see Moulin, 1988. See also Coughlin, 1990, and Demange, 1983). In the framework of this thesis, where the number of alternatives is usually infinite, this voting scheme is of no use.

\footnotetext{
${ }^{3}$ For a set $X,|X|$ denotes the cardinality of $X$.
} 
We call a relation $R$ on $A$ transitive if, for all $x, y, z \in A,(x, z) \in R$ whenever $(x, y) \in R$ and $(y, z) \in R$. If $P$ is a class of transitive preferences, strategy-proofness and intermediate strategy-proofness with respect to $P$ are equivalent, as is shown by the following lemma.

Lemma 1.1 Let $P$ be a class of transitive preferences on $A$. Then $\varphi: A^{N} \rightarrow$ $A$ satisfies $S P$ with respect to $P$ if and only if $\varphi$ satisfies ISP with respect to P.

Proof It suffices to show that SP with respect to $P$ implies ISP with respect to $P$. Let $\varphi$ be strategy-proof with respect to $P, R \in P, S \subset N$, and $p, q \in A^{N}$ such that $p(S) \subset \operatorname{best}(R)$ and $p={ }_{N \backslash S} q$. We will prove that $(\varphi(p), \varphi(q)) \in R$. Without loss of generality suppose $S=\{1,2, \ldots, l\}$. Take $q^{0}$ up to $q^{l}$ defined for all $i \in\{0, \ldots, l\}$ and $j \in N$ as follows:

$$
q^{i}(j)=\left\{\begin{array}{lll}
p(j) & \text { if } & j>i \\
q(j) & \text { if } & j \leq i
\end{array}\right.
$$

Observe that $q^{0}=p$ and $q^{l}=q$. Then it follows from strategy-proofness of $\varphi$ that for all $i \in S$ :

$\left(\varphi\left(q^{i-1}\right), \varphi\left(q^{i}\right)\right) \in R$. Hence, by the transitivity of $R,(\varphi(p), \varphi(q)) \in R$.

In lemma 1.1 the transitivity of $P$ is essential, as is shown by the following example.

Example 1.2 Let $A=\{a, b, c, d\}$. Let $P$ be a preference class on $A$ which contains only element, namely $R$. This preference is defined by

$$
R=\{(a, a),(b, b),(c, c),(d, d),(a, b),(a, c),(a, d),(b, c),(c, d),(d, b)\}
$$

So best $(R)=\{a\}, b$ is preferred to $c, c$ to $d$ and $d$ to $b$. Note the $R$ is not transitive: $(b, c) \in R$ and $(c, d) \in R$, but not $(b, d) \in R$.

Let $\varphi: A^{2} \rightarrow A$ be defined as follows. For all $x, y \in A$ :

$$
\begin{aligned}
\varphi(x, y) & =b \text { if } x, y \in\{a, b\} \\
& =d \text { if } x=y=d \\
& =c \text { otherwise. }
\end{aligned}
$$

If both agents report their bliss point, namely $a$, then $b$ will be the compromise point. If one of the agents manages to change the compromise point by not reporting $a$, the compromise point will be $c$. This alternative is worse 
than $b$, because $(c, b) \notin R$. So $\varphi$ satisfies SP, and even SSP with respect to $P$.

On the other hand, $\varphi(d, d)=d$. Since $(b, d) \notin R, d$ is better than $b$. So by deviating simultaneously from the ideal point they have in common, the agents can enforce a better compromise point. Consequently, $\varphi$ does not satisfy ISP.

Given an $n$-person voting scheme with $n \geq 2$, we can construct a voting scheme for less persons, by considering some subsets of $N$ as one agent. Many properties of the original scheme are inherited by the constructed scheme. So we have for instance:

Lemma 1.2 Let $P$ be a class of transitive preferences on $A$. Let $\varphi: A^{N} \rightarrow$ $A$ satisfy $S P$ with respect to $P$. Let $S, T, U$ be pairwise disjoint subsets of $N$. Let $p \in A^{N \backslash(S \cup T \cup U)}$. Let $\psi: A^{3} \rightarrow A$ be defined by $\psi(q)=$ $\varphi\left(\left\langle q(1)^{S}, q(2)^{T}, q(3)^{U}, p\right\rangle\right)$ for all $q \in A^{3}$. Then $\psi$ satisfies $S P$ with respect to $P$.

Proof Lemma 1.1 implies that $\varphi$ satisfies ISP with respect to $P$. Suppose $R \in P, i \in\{1,2,3\}, \tilde{q}, \hat{q} \in A^{3}$ are such that $\tilde{q}(i) \in$ best $(R)$ and $\tilde{q}(j)=\hat{q}(j)$ for $j \in\{1,2,3\} \backslash\{i\}$. Then by ISP, $\left(\varphi\left(\left\langle\tilde{q}(1)^{S}, \tilde{q}(2)^{T}, \tilde{q}(3)^{U}, p\right\rangle\right)\right.$ $\left.\varphi\left(\left\langle\hat{q}(1)^{S}, \hat{q}(2)^{T}, \hat{q}(3)^{U}, p\right\rangle\right)\right) \in R$. So, $(\psi(\tilde{q}), \psi(\hat{q})) \in R$. Consequently, $\psi$ satisfies $\mathrm{SP}$ with respect to $P$.

Special cases of this lemma are obtained by taking for instance $S=T=\emptyset$. Lemma 1.2 can be used to relate location problems with different numbers of agents. Many times we will obtain conclusions about some location problem by first studying a simpler problem.

We call a class of preferences $P$ standard, if

(i) all $R \in P$ are transitive,

(ii) $|\operatorname{best}(R)|=1$ for all $R \in P$,

(iii) $\cup_{R \in P} \operatorname{best}(R)=A$.

So if $P$ is standard, lemmas $\mathbf{1 . 1}$ and 1.2 can be applied. The preference classes we study in chapters 2,3 and 4 are standard. In chapter 5 and 6 we will study some nonstandard preference classes. For standard preference classes $P$, weak Pareto optimality implies unanimity: 
Lemma 1.3 Let $P$ be a standard class of preferences on $A$. Let $\varphi: A^{N} \rightarrow A$ satisfy WPO with respect to $P$. Then $\varphi$ satisfies $U N$.

Proof Let $x \in A$. Let $p=\left\langle x^{N}\right\rangle$. By assumption we can take $R \in P$ with $\operatorname{best}(R)=\{x\}$, so $(x, \varphi(p)) \in R$. Hence WPO implies that $(\varphi(p), x) \in R$. Since $x \in \operatorname{best}(R),(x, y) \in R$ for all $y \in A$. Hence by the transitivity of $R,(\varphi(p), y) \in R$ for all $y \in A$. So $\varphi(p) \in \operatorname{best}(R)$. Therefore, $\varphi(p)=x$. Consequently, $\varphi$ is unanimous.

Similarly, for standard classes of preferences, strategy-proofness implies a property named confirmatoriness. We call a scheme $\varphi: A^{N} \rightarrow A$ confirmatory if for all $p, q \in A^{N}$ with $q(i) \in\{p(i), \varphi(p)\}$ for all $i \in N$ we have that $\varphi(q)=\varphi(p)$.

Lemma 1.4 Let $P$ be a standard class of preferences on $A$. Let $\varphi: A^{N} \rightarrow A$ satisfy $S P$ with respect to $P$. Then $\varphi$ is confirmatory.

Proof Let $p, q \in A^{N}$ with $q(i) \in\{p(i), \varphi(p)\}$ for all $i \in N$. By assumption we can take $R \in P$ such that best $(R)=\{\varphi(p)\}$. Since $P$ is a class of transitive preferences, lemma 1.1 implies that $\varphi$ satisfies ISP with respect to $P$. Let $S=\{i \in N: q(i)=\varphi(p)\}$. Then $q=N \backslash S p$. So by ISP, $(\varphi(q), \varphi(p)) \in R$. Since $\varphi(p) \in \operatorname{best}(R),(\varphi(p), x) \in R$ for all $x \in A$. Hence, by transitivity, $(\varphi(q), x) \in R$ for all $x \in A$. So, $\varphi(q) \in \operatorname{best}(R)$. Therefore, $\varphi(q)=\varphi(p)$. Consequently, $\varphi$ is confirmatory.

An implication of this lemma is the equivalence of unanimity and surjectivity if strategy-proofness with respect to a standard preference class is assumed:

Corollary 1.1 Let $P$ be a standard class of preferences on $A$. Let $\varphi: A^{N} \rightarrow$ $A$ satisfy $S P$ with respect to $P$. Then $\varphi$ satisfies $U N$ if and only if $\varphi$ satisfies SURJ.

Proof It is obvious that UN implies SURJ. Now assume $\varphi$ satisfies SURJ. Let $a \in A$. It suffices to prove that $\varphi\left(\left\langle a^{N}\right\rangle\right)=a$. By surjectivity, we can take $p \in A^{N}$ with $\varphi(p)=a$. Lemma 1.4 implies that $\varphi$ is confirmatory. Consequently, $\varphi\left(\left\langle a^{N}\right\rangle\right)=a$.

We conclude this section by introducing some geometrical notations. For any subset $S \subset \mathbb{R}^{m}$ the convex hull of $S$, i.e. the smallest convex set containing $S$, will be denoted by conv $S$. Then conv $S$ is the set of all convex combinations 
of elements of $S$ (cf. Rockafellar, 1970, p. 12), i.e.,

$$
\begin{aligned}
\operatorname{conv} S= & \left\{x \in \mathbf{R}^{m}: \exists k \in \mathbf{N} ; \exists \lambda_{i} \in[0,1], s^{i} \in S \text { for } i=1, \ldots, k:\right. \\
& \left.x=\sum_{i=1}^{k} \lambda_{i} s^{i} \text { and } \sum_{i=1}^{k} \lambda_{i}=1\right\} .
\end{aligned}
$$

For any subset $S \subset \mathbb{R}^{m}$ the affine hull of $S$ will be denoted as aff $S$, i.e. (cf. Rockafellar, 1970, p. 7)

$$
\begin{aligned}
\text { aff } S= & \left\{x \in \mathbb{R}^{m}: \exists k \in \mathbf{N} ; \exists \lambda_{i} \in \mathbf{R}, s^{i} \in S \text { for } i=1, \ldots, k:\right. \\
& \left.\sum_{i=1}^{k} \lambda_{i}=1 \text { and } x=\sum_{i=1}^{k} \lambda_{i} s^{i}\right\} .
\end{aligned}
$$

For any subset $S \subset \mathbf{R}^{m}$ the linear hull of $S$ will be denoted as $\operatorname{span} S$, i.e. (cf. Rockafellar, 1970, pp. 5-7), $\operatorname{span} S=\operatorname{aff}(S \cup\{0\}$ ).

We will use the following notations for line segments. For $x, y \in \mathbf{R}^{m}$ we define $^{4}$ :

$$
\begin{aligned}
{[x, y] } & :=\operatorname{conv}\{x, y\} \text { (closed line segment) } \\
{[x, y) } & :=[x, y] \backslash\{y\} \text { (half open line segment) } \\
(x, y) & :=[x, y] \backslash\{x, y\} \text { (open line segment) } \\
{[x, y, \rightarrow) } & :=\left\{u \in \mathbf{R}^{m} \mid \exists \lambda \in[0, \infty): u=x+\lambda(y-x)\right\} \text { (closed halfine) } \\
(x, y, \rightarrow) & :=[x, y, \rightarrow) \backslash\{x\} \text { (open halfine) } \\
(\leftarrow, x, y, \rightarrow) & :=[x, y, \rightarrow) \cup[y, x, \rightarrow) \text { (line). }
\end{aligned}
$$

For $x, y, z \in \mathbb{R}^{m}$ we define:

$$
\Delta(x, y, z):=\operatorname{conv}\{x, y, z\}
$$

$\angle(x, y, z)$ denotes the (measure of the) angle between $z-y$ and $x-y$ for $x, y, z \in \mathbb{R}^{m}$ such that $x \neq y$ and $z \neq y$.

Furthermore, $(x, y) / /(z, w)$ means that the line segments $(x, y)$ and $(z, w)$ are parallel, i.e. $0, y-x$ and $w-z$ are collinear.

Sometimes we will allow parameters of schemes to be infinite. In view of this we define $\overline{\mathbf{R}}:=\mathbf{R} \cup\{-\infty, \infty\}$.

\footnotetext{
'In connection with preferences $(x, y)$ denotes an ordered pair. The notation should be clear from the context.
} 


\subsection{Voting schemes for location problems}

In section 1.2 we described the constant scheme and the dictatorial scheme. Both voting schemes are regarded to be undesirable. In this section we discuss some other voting schemes. Most of these will play important roles in this monograph.

\subsubsection{The mean prescheme}

This prescheme is defined by $\varphi(p)=\frac{1}{n} \sum_{i=1}^{n} p(i)$. By definition,

$$
\varphi(p) \in \operatorname{conv}\{p(i): i \in N\} \text { for all } p \in A^{N} \text {. }
$$

So if $A$ is convex, $\varphi$ is a scheme.

If $A$ is not convex, $\varphi$ can still be a scheme (for instance if $A=\mathbb{Q}^{m}$ ). Under some circumstances, which we will discuss later, (1.1) implies that $\varphi$ is Pareto optimal. In general however, $\varphi$ need not satisfy PO. Since $\varphi\left(\left\langle x^{N}\right\rangle\right)=x$ for all $x \in A, \varphi$ satisfies UN and SURJ. If $A$ is convex, $\varphi$ satisfies RC. If $A=\mathbf{R}^{m}$ and $P$ is an standard class of preferences, $\varphi$ is not strategy-proof with respect to $P$ (if agent $i$ reports $\sum_{j=1}^{n}(p(i)-p(j)$ ) instead of his true preference point, the scheme point will be at $p(i))$. Since $\varphi$ is not strategy-proof, it certainly does not satisfy ISP, CSP and SSP. Since summation is a commutative operator, $\varphi$ satisfies $\mathrm{AN}$. Note that $\varphi$ does not satisfy confirmatoriness.

\subsubsection{The median prescheme}

Let $k \in\{0,1,2, \ldots\}$ and let $x_{1}, x_{2}, \ldots, x_{2 k+1}$ be real numbers. The median of these numbers, denoted $\operatorname{med}\left(x_{1}, \ldots, x_{2 k+1}\right)$ is the real number $x$ with $\left|\left\{i: x_{i} \leq x\right\}\right| \geq k+1$ and $\left|\left\{i: x_{i} \geq x\right\}\right| \geq k+1$. For $m=1, n$ odd the median prescheme is defined by $\varphi(p)=\operatorname{med}(p(1), \ldots, p(n))$. By definition,

$$
\varphi(p) \in\{p(i): i \in N\} \text { for all } p \in A^{N} \text {. }
$$

Hence, $\varphi$ is always a scheme. Also by (1.2) $\varphi$ is Pareto optimal with respect to standard classes of preferences. It is easy to verify that $\varphi$ also satisfies $\mathrm{UN}$ and SURJ. If $A$ is convex, $\varphi$ also satisfies RC. Under conditions we will discuss later, $\varphi$ satisfies SP, ISP, CSP and SSP. The anonymity of $\varphi$ is obvious.

Consequently, the median scheme is attractive, but for $n$ even or $m>1$ it is not defined. 


\subsubsection{Coordinatewise median schemes}

These schemes are generalizations of the median scheme, and are defined for all $m, n \in \mathbf{N}$. They were previously introduced by Moulin (1980) for the case $m=1$, and by Border and Jordan (1983). Border and Jordan used the expression 'generalized median'. As there are many ways to generalize the median for the multidimensional case (see the references at the end of this subsection), we prefer the term 'coordinatewise median'.

Let $u^{1}, \ldots, u^{m} \in \mathbf{R}^{m}$ be linearly independent. Let $U$ be the $m \times m$ matrix with $u^{1}, \ldots, u^{m}$ as column vectors. Let $e^{j}$ be the $j$-th unit vector in $\mathbf{R}^{m}$. Then it is easy to verify that $x=\sum_{j=1}^{m}\left(e^{j^{T}} U^{-1} x\right) u^{j}$ for all $x \in \mathbb{R}^{m}$. So the $e^{j^{T}} U^{-1} x$ are the coordinates of $x$ with respect to the basis $\left\{u^{1}, \ldots, u^{m}\right\}$ of $\mathbb{R}^{m}$. Usually we will omit the transpose index ${ }^{T}$. Instead of $e^{j} U^{-1} x$ we will sometimes use the notation $\lambda_{j}(x)$.

Let $k \in \mathbb{N}$ with $k+n$ odd. Let $c^{i} \in \overline{\mathbf{R}}^{m}$ for $i=1, \ldots, k$. We define the coordinatewise median prescheme $\varphi$ with respect to $\left\{u^{1}, \ldots, u^{m}\right\}$ with $k$ constant points $\left\{c^{1}, \ldots, c^{k}\right\}$ as follows:

$$
\varphi(p)=\sum_{j=1}^{m} \operatorname{med}\left(e^{j} U^{-1} p(1), \ldots, e^{j} U^{-1} p(n), c_{j}^{1}, \ldots, c_{j}^{k}\right) u^{j} .
$$

The constant points $c^{i}$ are sometimes called phantom voters, (cf. Border and Jordan, 1983) because they seem to correspond to ideal points of imaginary agents (cf. example 1.3 below). However, they are just parameters of $\varphi$. For all $k \in \mathbb{N}$ with $k+n$ odd, each coordinatewise median prescheme coincides with a coordinate median prescheme with $n+1$ constant points. For instance, if $c^{1}, \ldots, c^{n+1} \in \overline{\mathbf{R}}^{m}$ are such that

$$
\left|\left\{i \in\{1, \ldots, n+1\}: c_{j}^{i}=-\infty\right\}\right|=\left|\left\{i \in\{1, \ldots, n+1\}: c_{j}^{i}=\infty\right\}\right|=\frac{n+1}{2}
$$
for $j=1, \ldots, m$,

$\varphi$ coincides with the coordinatewise median prescheme with respect to $\left\{u^{1}\right.$, $\left.\ldots, u^{m}\right\}$ without constant points, i.e.

$$
\varphi(p)=\sum_{j=1}^{m} \operatorname{med}\left(e^{j} U^{-1} p(1), \ldots ; e^{j} U^{-1} p(n)\right) u^{j}
$$

Note that this prescheme is only defined for $n$ odd.

For $m=1, n$ odd, this prescheme coincides with the median prescheme. So the coordinatewise median prescheme without constant points is a gener- 
alization of the median scheme, whereas this prescheme can be generalized to the coordinatewise median prescheme with $n+1$ constant points.

If all $c^{i}$ are equal, $\varphi\left(p ; c^{1}, \ldots, c^{n+1}\right)=c^{1}$, so $\varphi$ coincides with the constant scheme.

It is easy to verify that the coordinatewise median prescheme without phantom voters satisfies UN, AN and SURJ. RC is satisfied if $A$ is convex. Under certain conditions $\varphi$ satisfies SP and PO. We postpone further discussion on these matters until chapter 2 , where coordinatewise median schemes play an important role.

Every coordinatewise median scheme with respect to one basis can easily be transformed to a coordinatewise median scheme with respect to another basis. We have the following lemmas:

Lemma 1.5 Let $\varphi:\left(\mathbb{R}^{m}\right)^{N} \rightarrow \mathbb{R}^{m}$. Let $V$ be a nonsingular $m \times m$ matrix. Then $\varphi$ is the coordinatewise median scheme with respect to the basis $\left\{u^{j}\right.$ : $j, \ldots, m\}$ and constant points $\left\{c^{i}: i=1, \ldots, k\right\}$ if and only if $V \circ \varphi \circ V^{-1}$ is the coordinatewise median scheme with respect to the basis $\left\{V u^{j}: j=\right.$ $1, \ldots, m\}$ and constant points $\left\{c^{i}: i=1, \ldots, k\right\}$.

Proof Let

$$
\psi=V \circ \varphi \circ V^{-1} .
$$

Let $\varphi$ be the coordinatewise median scheme with respect to the basis $\left\{u^{j}\right.$ : $j=1, \ldots, m\}$ and constant points $\left\{c^{i}: i=1, \ldots, k\right\}$. It suffices to prove that $\psi$ is also such a scheme. Let $U$ be the $m \times m$ matrix with $u^{1}, \ldots, u^{m}$ as column vectors. Then $\varphi(p)=\sum_{j=1}^{m} \operatorname{med}\left(e^{j} U^{-1} p(1), \ldots, e^{j} U^{-1} p(n)\right.$, $\left.c_{j}^{1}, \ldots, c_{j}^{k}\right) u^{j}$ for all $p \in\left(\mathbb{R}^{m}\right)^{N}$. Hence,

$$
\begin{aligned}
\psi(p)= & \sum_{j=1}^{m} \operatorname{med}\left(e^{j} U^{-1} V^{-1} p(1), \ldots, e^{j} U^{-1} V^{-1} p(n),\right. \\
& \left.c_{j}^{1}, \ldots, c_{j}^{k}\right) V u^{j} \text { for all } p \in\left(\mathbf{R}^{m}\right)^{N} .
\end{aligned}
$$

Let $w^{j}=V u^{j}$ and $W$ be the $m \times m$ matrix with $w^{1}, \ldots, w^{m}$ as column vectors. Then $W=V U$. So, $W^{-1}=U^{-1} V^{-1}$, and by (1.4):

$$
\begin{aligned}
\psi(p)= & \sum_{j=1}^{m} \operatorname{med}\left(e^{j} W^{-1} p(1), \ldots, e^{j} W^{-1} p(n), c_{j}^{1}, \ldots, c_{j}^{k}\right) w^{j} \\
& \text { for all } p \in\left(\mathbf{R}^{m}\right)^{N} .
\end{aligned}
$$

Consequently, $\psi$ is the coordinatewise median scheme with respect to $\left\{V u^{j}\right.$ : $j=1, \ldots, m\}$ and constant points $\left\{c^{i}: i=1, \ldots, k\right\}$. 
Lemma 1.6 Let $\varphi:\left(\mathbf{R}^{m}\right)^{N} \rightarrow \mathbb{R}^{m}$ be the coordinatewise median scheme with respect to the basis $\left\{u^{j}: j=1, \ldots, m\right\}$ and constant points $\left\{c^{i}: i=\right.$ $1, \ldots, k\}$. Let $\psi:\left(\mathbf{R}^{m}\right)^{N} \rightarrow \mathbf{R}$ be the coordinatewise median scheme with respect to the basis $\left\{w^{j}: j=1, \ldots, m\right\}$ and constant points $\left\{c^{i}: i=1, \ldots, k\right\}$. Then there is a nonsingular $m \times m$ matrix $V$ such that $\psi=V^{-1} \circ \varphi \circ V$.

Proof Let $U$ be the $m \times m$ matrix with $u^{1}, \ldots, u^{m}$ as column vectors. Since the $u^{j}$ are linearly independent, $U$ is invertible. Let $W$ be the $m \times m$ matrix with $w^{1}, \ldots, w^{m}$ as columns vectors. Similarly, $W$ is invertible. Let $V=W U^{-1}$. Then $V^{-1}=U W^{-1}$. Furthermore, $V u^{j}=W U^{-1} u^{j}=w^{j}$ for $j=1, \ldots, m$. Hence, by lemma $1.5, V^{-1} \circ \varphi \circ V$ is the coordinatewise median scheme with respect to the basis $\left\{V u^{j}: j=1, \ldots, m\right\}$ and constant points $\left\{c^{i}: i=1, \ldots, k\right\}$. Consequently, $\psi=V^{-1} \circ \varphi \circ V$.

The following example may explain why some authors speak of 'phantom voters', where we speak of constant points.

Example 1.3 A house of parliament has 150 members. To accept a new law, they need a two third majority. This can be modelled as follows:

Let $n=150, m=1, A=\{0,1\}$. Let $\psi: U^{N} \rightarrow \mathbf{R}$ be the coordinatewise median prescheme with respect to $\{1\}$ (note we do not have much freedom in choosing a basis in $\mathbf{R}^{1}$ ) with 49 constant points $c^{i}$ such that $c^{i}=0$ for $i=1, \ldots, 49$. In this context, 0 means 'no' and 1 means 'yes' in relation to acceptance of the new law. Since for all profiles $p \in U^{N}, \varphi(p) \in A, \varphi$ is a scheme. Furthermore, $\varphi(p)=\operatorname{med}\left(p(1), \ldots, p(150), c^{1}, \ldots, c^{49}\right)$. Hence,

$$
\begin{aligned}
\varphi(p) & =0 \text { if }|\{i \in N: p(i)=1\}| \leq 99 \\
& -1 \text { if }|\{i \in N: p(i)=1\}| \geq 100 .
\end{aligned}
$$

So indeed a two third majority is needed to accept the law. It looks as if the usual majority rule is used, with 49 extra voters present who all vote against acceptance. Since these voters are not 'real', they are called 'phantom voters'.

There are other ways to generalize the concept of the median into higher dimensional settings. Small (1990) gives a survey on statistical applications of these generalized medians. Peters and Van der Stel (1990) discuss the application of a generalized median on multiperson multicriteria decision making. 


\subsubsection{Coordinatewise vetopreschemes}

Let $\left\{u^{j}: j=1, \ldots, m\right\}$ be a basis of $\mathbf{R}^{m}$. Let $e^{j}$ be the $j$-th unit vector in $\mathbf{R}^{m}, j=1, \ldots, m$. Let $U$ be the $m \times m$ matrix with $u^{1}, \ldots, u^{m}$ as column vectors.

A map $\gamma: 2^{N} \rightarrow \overline{\mathbf{R}}^{m}$ is an assignment of disagreements if, for all $t \in M$ and $S \subset T \subset N, \gamma(\emptyset)_{t}>-\infty, \gamma(N)_{t}<\infty$, and $\gamma(S)_{t} \geq \gamma(T)_{t}$. To such an assignment of disagreements $\gamma$ a prescheme $\varphi_{\gamma}$ with respect to $\left\{u^{j}: j=\right.$ $1, \ldots, m\}$ is associated as follows:

$$
\varphi_{\gamma}(p)=\sum_{j=1}^{m} \min _{S \subset N}\left\{\max _{i \in S}\left\{e^{j} U^{-1} p(i) \vee \gamma_{j}(S)\right\}\right\} u^{j}
$$

for all $p \in A^{N}$. $\varphi_{y}$ is called a coordinatewise vetoprescheme with respect to $\left\{u^{j}: j=1, \ldots, m\right\}$.

Whether $\varphi_{\gamma}$ is a scheme, depends on $A$ as well as on $\gamma$ and the $u^{j}$.

If $\varphi_{\gamma}$ is a scheme, it is called a coordinatewise veto scheme. If $\gamma(S)$ depends only on $|S|$ for all $S \subset N, \varphi_{\gamma}$ coincides with a coordinatewise median prescheme with respect to $\left\{u^{1}, \ldots, u^{m}\right\}$ and constant points $c^{1}, \ldots, c^{n+1}$ such that $c_{j}^{i}=\gamma(\{1, \ldots, i-1\})_{j}$ for $i=1, \ldots, n+1$ and $j=1, \ldots, m$ (cf. lemma 5.5). It is easy to verify that every coordinatewise median (pre)scheme can be written as a coordinate veto(pre)scheme.

If there is an $i \in N$ such that for all $S \subset N: \gamma(S)_{j}=-\infty$ if $i \in S$ and $\gamma(S)_{j}=\infty$ if $i \notin S$ for all $j \in\{1, \ldots, m\}, \varphi_{\gamma}$ coincides with the dictatorial scheme with dictator $i$. So, except for the mean scheme, all the scheme concepts we have discussed are special cases of coordinatewise pre schemes. A standard coordinatewise veto(pre)scheme is a coordinatewise veto(pre)scheme with respect to the standard basis $\left\{e^{j}: j=1, \ldots, m\right\}$. These schemes will play an important role in chapter 5 . Coordinatewise veto(pre)schemes were studied before by Moulin (1980) and Border and Jor$\operatorname{dan}(1983)$ 



\section{Chapter 2}

\section{Pareto Optimality and Strictly Convex Norms}

This chapter is based on Peters, Van der Stel and Storcken (1993a).

\subsection{Introduction}

In a location problem, $n$ agents each report a point in a subset $A$ of an $m$ dimensional Euclidean space. A scheme assigns to each $n$-tuple of reported points a compromise point in $A$. This compromise point is evaluated by each agent individually in terms of his preferences over the points of $A$.

In this chapter it is assumed that each agent's preference for a compromise point is determined by the distance of the compromise point to the agent's most preferred point (ideal point, bliss point). The same metric is assumed for all agents. Consequently, for each agent the preferences for a compromise point are determined by his ideal point. Instead of formulating axioms like Pareto optimality and strategy-proofness with respect to classes of preferences, we now can formulate these axioms with respect to the chosen metric (or the norm by which this metric is induced). This will be done in section 2.3 .

The topics of section 2.2 are purely geometrical. First we define norms on $\mathbf{R}^{m}$, and the subclass of strictly convex norms. Lemma 2.1 gives some properties of norms which are characteristic for strictly convex norms.

In an inner product space two vectors are called orthogonal if their inner product is zero. A set is called orthogonal if its elements are pairwise orthogonal. In the remainder of section 2.2 we discuss three ways of defining 
orthogonality of sets in terms of norms, namely $\|\cdot\|$-orthogonality, weak $\|\cdot\|$-orthogonality and $\|\cdot\|$-orthogonality in the sense of Birkhoff (1935). The advantage of these $\|\cdot\|$-orthogonality concepts is that they are also suitable for norms which are not induced by an inner product. In lemma 2.2 we show that these three $\|\cdot\|$-orthogonality concepts are equivalent to the usual orthogonality concept in the case that $\|\cdot\|$ is induced by an inner product.

In general, we have that $\|\cdot\|$-orthogonality implies weak $\|\cdot\|$-orthogonality, whereas weak $\|\cdot\|$-orthogonality implies $\|\cdot\|$-orthogonality in the sense of Birkhoff. We give some examples of norms which show that these two implications are strict. We investigate the existence of a (weakly) $\|\cdot\|$-orthogonal basis (in the sense of Birkhoff) of $\mathbb{R}^{m}$, given a certain norm $\|\cdot\|$. Lemma 2.5 shows that a weakly $\|\cdot\|$-orthogonal basis of $\mathbb{R}^{m}$ always exists. The existence of a $\|\cdot\|$-orthogonal basis can only be guaranteed for $m \in\{1,2\}$ (see corollary 2.3 and example 2.5). Later on in this chapter (theorem 2.6) we will show that this result is sufficient to guarantee the existence of a voting scheme on $\mathbf{R}^{2}$ which is anonymous, and Pareto optimal and strategy-proof with respect to $\|\cdot\|$, if $\|\cdot\|$ is an arbitrary strictly convex norm on $\mathbb{R}^{2}$.

In section 2.4 the two person location problem is handled. One of the conclusions (cf. theorem 2.2) is that only dictatorial schemes satisfy SP and SPO with respect to a norm, under the assumption that $A$ is open. Furthermore, an impossibility result is obtained for the case where the number of players is even. Theorem 2.3 states that in this case there are no schemes satisfying anonymity, strong Pareto optimality and strategy-proofness. For strictly convex norms, SPO can be relaxed to PO or WPO.

In sections $2.5,2.6$ and 2.7 we assume that the set of alternatives $A$ coincides with $\mathbf{R}^{m}$. In section 2.5 we study three person location problems in $\mathbf{R}^{m}$. On the schemes two axioms are imposed, namely strategy-proofness and Pareto optimality with respect to some strictly convex norm. An important consequence of these axioms will be the coordinatewise continuity of the schemes. The obtained results will be used in section 2.6, where we focus on three person location problems in $\mathbf{R}^{2}$. Theorem 2.4 gives a complete characterization of all schemes satisfying anonymity, strategy-proofness and Pareto optimality. This result is generalized in section 2.7 for an arbitrary odd number of agents. Theorem 2.5 states that such a scheme must coincide with a constant point free coordinatewise median scheme with respect to a || $\|$-orthogonal basis. On the other hand, every coordinatewise median scheme is anonymous, and Pareto optimal and strategy-proof with respect to $\|\cdot\|$. The existence of such a scheme is guaranteed by theorem 2.6. These 
result cannot be generalized for location problems on $\mathbf{R}^{m}$, with $m \geq 3$. It depends on the norm $\|\cdot\|$ whether there exist schemes which are anony mous, Pareto optimal and strategy-proof.

In section 2.8 we search for schemes which are coalitionally strategyproof and Pareto optimal. Theorem 2.7 asserts that only dictatorial schemes satisfy these axioms. As is stated by corollaries 2.8 and 2.9, Pareto optimality can be weakened to unanimity or surjectivity, to obtain the same result. This shows that coalitional strategy-proofness is a far more restrictive requirement than strategy-proofness.

Section 2.9 concludes.

\subsection{Norms, strict convexity and orthogonality}

In this section we discuss norms on $\mathbb{R}^{m}$. A subclass of norms is the class of strictly convex norms. These norms have attractive properties, which will play an important role later on. Some alternative characterizations of strict convexity of a norm are discussed. Since the usual definition of orthogonality is in terms of an inner product, this definition is not applicable in the case of norms which are not induced by an inner product. We will introduce three generalizations of this concept. In inner product spaces there are infinitely many orthonormal sets with cardinality $m$. In the generalized case such sets need not even exist. Some lemmas and examples will clarify these matters. At the end we will give an alternative characterization of orthogonality which only holds for strictly convex norms.

A norm on $\mathbb{R}^{m}$ is a real-valued function on $\mathbb{R}^{m}$ whose value at an $x \in \mathbb{R}^{m}$ usually is denoted by $\|x\|$, (read "norm of $x$ ") and which has the following properties for arbitrary $\alpha \in \mathbb{R}, x, y \in \mathbb{R}^{m}$ :

(i) $\|x\| \geq 0$

(ii) $\|x\|=0$ if and only if $x=0$

(iii) $\| \alpha x||=|\alpha||| x||$

(iv) $\|x+y\| \leq\|x\|+\|y\|$ (triangular inequality).

$\|x\|$ can be interpreted as the "length" of vector $x$. A norm $\|\cdot\|$ on $\mathbb{R}^{m}$ defines a metric $\delta$ on $\mathbf{R}^{m}$ which is given by $\delta(x, y) \equiv\|x-y\|$ and is called the metric induced by the norm $\|\cdot\|$ (cf. Kreyszig, 1978, p. 59).

The unit ball of a norm $\|\cdot\|$ on $\mathbf{R}^{m}$ is the set $\left\{x \in \mathbf{R}^{m}:\|x\| \leq 1\right\}$. This set is convex and symmetric around the origin. 
A norm $\|\cdot\|$ on $\mathbf{R}^{m}$ is called strictly convex if for all $x, y \in \mathbf{R}^{m}$ such that $\|x\| \leq 1,\|y\| \leq 1$ and $x \neq y$ we have $\|x+y\|<2$ (Cf. Kreyszig, 1978, p. 332). So a norm $\|\cdot\|$ is strictly convex if and only if its unit sphere (i.e., the set $\left\{x \in \mathbf{R}^{m}:\|x\|=1\right\}$ ) contains no line segments which consists out of more than one point.

A metric on $\mathbb{R}^{m}$ will also be called strictly convex if it is induced by a strictly convex norm.

Examples of strictly convex norms on $\mathbb{R}^{m}$ are all p-norms $\|\cdot\|_{p}$ for $p \in(1, \infty)$, defined by

$$
\|x\|_{p} \equiv\left(\sum_{i=1}^{m}\left|x_{i}\right|^{p}\right)^{1 / p}
$$

(See figure 2.1.)

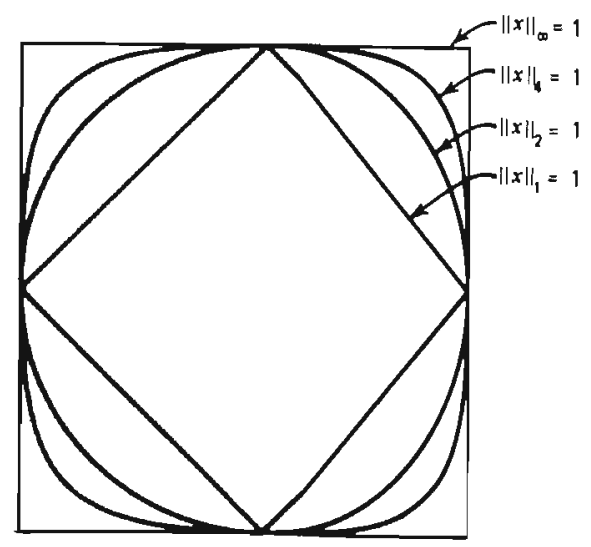

Figure 2.1: Unit spheres of some p-norms $(m=2)$

In particular, the Euclidean norm $(p=2)$ is strictly convex. Also norms which are induced by an inner product $\langle\cdot, \cdot\rangle$ on $\mathbf{R}^{m}$, i.e., $\|x\| \equiv \sqrt{\langle x, x\rangle}$ (cf. section 3.2), are strictly convex (for a proof we refer to Kreyszig, 1978, p. 333). It follows straightforwardly from the definition of a norm, that all norms on $\mathbb{R}^{1}$ have the structure $\|x\| \equiv \alpha\left|x_{1}\right|$, where $\alpha$ is a positive scalar. 
It is easy to verify that all these norms are strictly convex.

Norms on $\mathbf{R}^{m}$ ( $m \geq 2$ ) of which the unit ball is polyhedral, are not strictly convex. Examples of such norms are the maximumnorm $\|\cdot\|_{\infty}$ (i.e. $\left.\|x\|_{\infty}=\max _{j=1, \ldots, m}\left|x_{j}\right|\right)$ and the sum norm $\|\cdot\|_{1}$ (i.e. $\left.\|x\|_{1}=\sum_{j=1}^{m}\left|x_{j}\right|\right)$.

The following lemma gives some alternative characterizations of strict convexity.

Lemma 2.1 Let $\|\cdot\|$ be a norm on $\mathbb{R}^{m}$. Then the following assertions are equivalent:

(i) $\|\cdot\|$ is strictly convex.

(ii) For all $x, y, z \in \mathbb{R}^{m}$ such that $\|y\| \leq 1,\|z\| \leq 1$ and $x \in(y, z)$ we have $\|x\|<1$.

(iii) For all $x, y, z \in \mathbf{R}^{m}$ such that $\|y\| \leq\|z\|$ and $x \in(y, z)$ we have $\|x\|<\|z\|$.

(iv) For all $x, y, z \in \mathbb{R}^{m}$ such that $\|x\| \geq\|z\|$ and $x \in(y, z)$ we have $\|y\|>\|z\|$.

(v) For all $x, y, z \in \mathbb{R}^{m}$ such that $x \notin[y, z],\|x-y\|+\|x-z\|>\|y-z\|$.

Proof (i) $\Rightarrow$ (ii) Let $\|\cdot\|$ be strictly convex.

Let $x, y, z \in \mathbb{R}^{m}$ such that $\|y\| \leq 1,\|z\| \leq \mathbf{1}$ and $x \in(y, z)$. W.l.o.g. ${ }^{1}$ assume that $\|x-z\| \leq\|x-y\|$. Hence, $2 x-z \in[y, z]$. Let $\lambda \in[0,1]$ with $2 x-z=$ $\lambda y+(1-\lambda) z$. Hence, $\|2 x-z\|=\|\lambda y+(1-\lambda) z\| \leq \lambda\|y\|+(1-\lambda)|| z \| \leq 1$. Therefore, by the strict convexity of $\|\cdot\|,\|x\|<1$.

(ii) $\Rightarrow$ (iii) Let $x, y, z \in \mathbb{R}^{m}$ with $\|y\| \leq\|z\|$ and $x \in(y, z)$. Then $(y, z) \neq \emptyset$. Since $\|y\| \leq\|z\|$ this implies $\|z\| \neq 0$. Let $\tilde{x}=\frac{x}{\|z\|}, \tilde{y}=\frac{y}{\|z\|}$ and $\tilde{z}=\frac{z}{\|z\|}$. Then $\|\tilde{y}\| \leq 1$ and $\|\tilde{z}\|=1$. Furthermore, $\tilde{x} \in(\tilde{y}, \tilde{z})$. So (ii) implies that $\|\tilde{x}\|<1$. Consequently, $\|x\|<\|z\|$.

(iii) $\Leftrightarrow$ (iv) This equivalence is evident.

(iii) $\Rightarrow$ (v) Let $a, b, c \in \mathbb{R}^{m}$ be such that $c \notin[a, b]$. Suppose $\|a-c\|+\|b-c\| \leq$ $\|a-b\|$. Hence, $\|a-b\|>0$. By the triangular inequality we have $\|a-c\|+\|b-c\|=\|a-b\|$. Let $d=a+\frac{\|a-c\|}{\|a-b\|}(b-a)$. Hence, $d \in[a, b]$, $\|d-a||=\| a-c||$ and $\|d-b\|=\left\|a-b+\frac{\| a-c \mid}{\| a-b||}(b-a)\right\|=\|a-b||-\| a-c \|=$

\footnotetext{
${ }^{1}$ Without loss of generality
} 
$\|b-c\|$. Let $u=\frac{1}{2} c+\frac{1}{2} d$. Since $a-u \in(a-d, a-c)$ we have by (iii) that $\|a-u\|<\|a-c\|$. Similarly, $\|b-u\|<\|b-c\|$. Hence, $\|a-u\|+\|b-u\|<\|a-b\|$. This contradicts the triangular inequality for norms. Consequently, $\|a-c\|+\|b-c\|>\|a-b\|$.

(v) $\Rightarrow$ (i) Let $a, b \in \mathbf{R}^{m}$ be such that $\|a\| \leq 1,\|b\| \leq 1$ and $a \neq b$. If $a \in[0, a+b]$ then $\|a+b\|<2 \max (\|a\|,\|b\|) \leq 2$ and we are done. Now assume $a \notin[0, a+b]$. Hence by (iv), $\|a-0\|+\|a-(a+b)\|>\|a+b\|$. Hence, $\|a+b\|<\|a\|+\|b\| \leq 2$.

In an inner product space $Z$ the usual definition of orthogonality is as follows. Two vectors $x, y \in Z$ are orthogonal if the inner product ${ }^{2}\langle x, y\rangle$ is zero. An orthogonal set $X$ in an inner product space $Z$ is a subset $X \subset Z$ whose elements are pairwise orthogonal.

In this chapter we consider norms in $\mathbb{R}^{m}$ which are not necessarily induced by an inner product ${ }^{3}$ (for instance all $p$-norms with $p \neq 2$ are not induced by an inner product if $m>1$ ). In the remainder of this section we discuss some alternative definitions of orthogonality of sets which are suitable for all kind of norms on $\mathbb{R}^{m}$ (for a recent survey on properties of other kinds of orthogonality concepts on normed linear spaces, we refer to Alonso and Benítez (1988)).

Let $\|\cdot\|$ be a norm on $\mathbb{R}^{m}$. Let $x, y \in \mathbf{R}^{m}$. Then $x$ is $\|\cdot\|$-orthogonal to $y$ in the sense of Birkhoff (1935) if $\|x\| \leq\|x+\lambda y\|$ for all $\lambda \in \mathbb{R}$. We call a set $X \subset \mathbf{R}^{m}\|\cdot\|$-orthogonal in the sense of Birkhoff if all its elements are pairwise $\|\cdot\|$-orthogonal in the sense of Birkhoff.

The following example shows that if $y \in \mathbf{R}^{m}$ is $\|\cdot\|$-orthogonal to $z \in \mathbf{R}^{m}$ in the sense of Birkhoff, this does not imply that $z$ is $\|\cdot\|$-orthogonal to $y$ in the sense of Birkhoff.

Example 2.1 Let $\|\cdot\|_{\infty}$ denote the maximum norm on $\mathbf{R}^{2}$, i.e., $\|x\|_{\infty} \equiv$ $\max \left\{\left|x_{1}\right|,\left|x_{2}\right|\right\}$. Let $y=\left(\begin{array}{l}1 \\ 1\end{array}\right)$ and $z=\left(\begin{array}{l}1 \\ 0\end{array}\right)$. (See figure 2.2.) Then for all $\lambda \in \mathbb{R}$ we have that $\|y+\lambda z\|_{\infty}=\max \{|1+\lambda|, 1\} \geq 1=\|y\|_{\infty}$. Hence, $y$ is $\|\cdot\|_{\infty}$-orthogonal to $z$ in the sense of Birkhoff. On the other hand, $\left\|z-\frac{1}{2} y\right\|_{\infty}=\left\|\left(\begin{array}{c}\frac{1}{2} \\ -\frac{1}{2}\end{array}\right)\right\|_{\infty}=\frac{1}{2}<1=\|z\|_{\infty}$. So $z$ is not $\|\cdot\|_{\infty}$-orthogonal to $y$ in the sense of Birkhoff. From figure 2.1 it is also easy to see that the

\footnotetext{
${ }^{2}$ In section 3.2 , inner products are discussed in more detail.

${ }^{3} \mathrm{~A}$ norm $\|\cdot\|$ on $\mathbb{R}^{m}$ is induced by an inner product $\langle\cdot, \cdot\rangle$ on $\mathbb{R}^{m}$ if $\|x\| \equiv \sqrt{\langle x, x\rangle}$.
} 


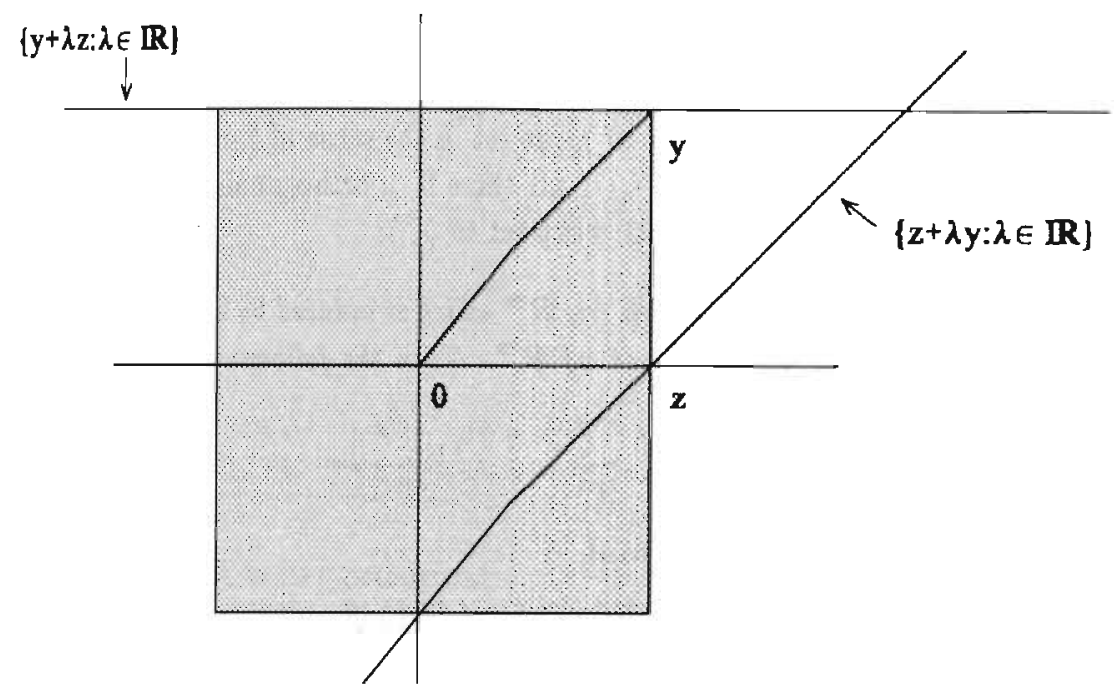

Figure 2.2: Example 2.1

set $\{z+\lambda y: \lambda \in \mathbf{R}\}$ intersects the interior of the unit ball of $\|\cdot\|_{\infty}$ (the shaded area in the picture).

Let $\|\cdot\|$ be a norm on $\mathbb{R}^{m}$. Let $k \in \mathbb{N}, x^{j} \in \mathbf{R}^{m}(j=1, \ldots, k)$. Let $X=\left\{x^{j}: j=1, \ldots, k\right\}$ with $|X|=k$. Then we call $X$ :

$\|\cdot\|$-orthogonal if for all $\lambda_{j} \in \mathbb{R}(j=1, \ldots, k)$, and all $l \in$ $\{1, \ldots, k\}$ we have that

$$
\left\|\sum_{\substack{j=1 \\ j \neq l}}^{k} \lambda_{j} x^{j}\right\| \leq\left\|\sum_{j=1}^{k} \lambda_{j} x^{j}\right\| ;
$$

weakly $\|\cdot\|$-orthogonal if for all $\lambda_{j} \in \mathbf{R}(j=1, \ldots, k)$, and all $i \in\{1, \ldots, k\}$ we have that

$$
\left\|\lambda_{i} x^{i}\right\| \leq\left\|\sum_{j=1}^{k} \lambda_{j} x^{k}\right\| .
$$

It is easy to verify that if $X \subset \mathbb{R}^{m}$ is (weakly) $\|\cdot\|$-orthogonal (in the sense of Birkhoff), and $Y \subset X$, then the set $Y$ is also (weakly) $\|\cdot\|$-orthogonal (in the sense of Birkhoff). 
It is easy to see that $\|\cdot\|$-orthogonality of a set in $\mathbf{R}^{m}$ implies weak $\|\cdot\|$-orthogonality of that set, whereas weak $\|\cdot\|$-orthogonality of a set in $\mathbf{R}^{m}$ implies $\|\cdot\|$-orthogonality of that set in the sense of Birkhoff. If $\|\cdot\|$ is induced by an inner product $\langle\cdot, \cdot\rangle$, all three $\|\cdot\|$-orthogonality concepts are equivalent to orthogonality with respect to $\langle\cdot, \cdot\rangle$ :

Lemma 2.2 Let $\|\cdot\|$ be a norm on $\mathbf{R}^{m}$ which is induced by an inner product $\langle\cdot, \cdot\rangle$ on $\mathbb{R}^{m}$. Let $X$ be a subset of $\mathbb{R}^{m}$. Then the following assertions are equivalent:

(i) $X$ is $\|\cdot\|$-orthogonal.

(ii) $X$ is weakly $\|\cdot\|$-orthogonal.

(iii) $X$ is $\|\cdot\|$-orthogonal in the sense of Birkhoff.

(iv) $X$ is orthogonal with respect to $\langle\cdot, \cdot\rangle$.

Proof Let $k=|X|$ and $x^{j} \in \mathbf{R}^{m}(j=1, \ldots, k)$ with $X=\left\{x^{j}: j=1, \ldots, k\right\}$.

(i) $\Rightarrow$ (ii) Straightforward.

(ii) $\Rightarrow$ (iii) Straightforward.

(iii) $\Rightarrow$ (iv) Assume $X$ is $\|\cdot\|$-orthogonal in the sense of Birkhoff. Let $x, y \in X, x \neq y$. It is sufficient to prove that $\langle x, y\rangle=0$. For all $\alpha \in \mathbf{R}$ we have $\|x\| \leq\|x+\alpha y\|$. Hence, for all $\alpha \in \mathbf{R}$ we have

$$
\begin{aligned}
\|x\|^{2} & \leq\|x+\alpha y\|^{2} \\
& =\langle x+\alpha y, x+\alpha y\rangle \\
& =\|x\|^{2}+2 \alpha\langle x, y\rangle+\alpha^{2}\|y\|^{2} .
\end{aligned}
$$

So for all $\alpha \in \mathbf{R}, 0 \leq 2 \alpha\langle x, y\rangle+\alpha^{2}\|y\|^{2}$. If $\|y\|=0$, then this implies that $\langle x, y\rangle=0$. If $\|y\|=0$, then by substituting $\frac{-\langle x, y\rangle}{\|y\|^{2}}$ for $\alpha$, we obtain $0 \leq \frac{-(\langle x, y\rangle)^{2}}{\|y\|^{2}}$. Consequently, $\langle x, y\rangle=0$.

(iv) $\Rightarrow$ (i) Assume that $X$ is orthogonal (with respect to $\langle\cdot, \cdot)$ ). For all $l \in\{1, \ldots, k\}, \lambda_{j} \in \mathbf{R}(j=1, \ldots, k)$ we have that:

$$
\left\|\sum_{j=1}^{k} \lambda_{j} x^{j}\right\|^{2}=\left\langle\sum_{j=1}^{k} \lambda_{j} x^{j}, \sum_{j=1}^{k} \lambda_{j} x^{j}\right\rangle
$$




$$
\begin{aligned}
& =\sum_{j=1}^{k} \lambda_{j}^{2}\left\langle x^{j}, x^{j}\right\rangle \geq \sum_{\substack{j=1 \\
j \neq l}}^{k} \lambda_{j}^{2}\left\langle x^{j}, x^{j}\right\rangle \\
& =\left\|\sum_{\substack{j=1 \\
j \neq l}}^{k} \lambda_{j} x^{j}\right\|^{2}
\end{aligned}
$$

and hence

$$
\left\|\sum_{j=1}^{k} \lambda_{j} x^{j}\right\| \geq\left\|\sum_{\substack{j=1 \\ j \neq l}} \lambda_{j} x^{j}\right\|
$$

Consequently, $X$ is $\|\cdot\|$-orthogonal.

It follows from the definitions, that the three $\|\cdot\|$-orthogonality concepts are equivalent for sets with cardinality 2 . With respect to sets with higher cardinality this need not be the case as is shown by the next two examples (see also example 2.4). Example 2.2 shows that $\|\cdot\|$-orthogonality may be more restrictive than weak $\|\cdot\|$-orthogonality.

Example $2.2^{4}$ Let $m=3$. Let $\|\cdot\|_{1}$ be the sum norm on $\mathbb{R}^{3}$, i.e., $\|x\|_{1} \equiv$ $\left|x_{1}\right|+\left|x_{2}\right|+\left|x_{3}\right|$. Let $X=\left\{x^{1}, x^{2}, x^{3}\right\}$, with $x^{1}=\left(\begin{array}{l}1 \\ 1 \\ 0\end{array}\right), x^{2}=\left(\begin{array}{l}0 \\ 1 \\ 1\end{array}\right)$ and $x^{3}=\left(\begin{array}{l}1 \\ 0 \\ 1\end{array}\right)$. Since $\left\|x^{1}+x^{2}\right\|_{1}=4>2=\left\|x^{1}+x^{2}+x^{3}\right\|_{1}, X$ is not $\|\cdot\|_{1}$-orthogonal.

On the other hand, we have for all $l \in\{1,2,3\}$ and all $\lambda_{1}, \lambda_{2}, \lambda_{3} \in \mathbf{R}$ that $\left\|\sum_{j=1}^{3} \lambda_{j} x^{j}\right\|_{1}=\left|\lambda_{1}+\lambda_{3}\right|+\left|\lambda_{1}+\lambda_{2}\right|+\left|\lambda_{2}+\lambda_{3}\right| \geq 2\left|\lambda_{l}\right|=\left\|\lambda_{l} x^{l}\right\|_{\mathbf{1}}$. Consequently, $X$ is weakly $\|\cdot\|_{1}$-orthogonal. Finally, it should be noted that

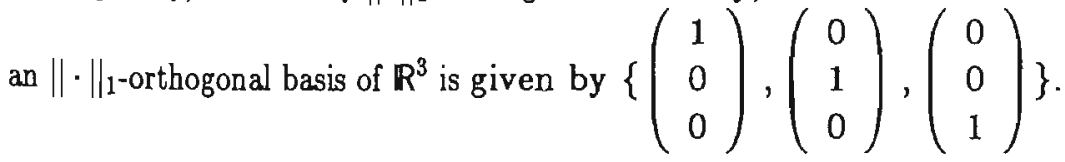

Example 2.3 shows that weak $\|\cdot\|$-orthogonality may be more restrictive than $\|\cdot\|$-orthogonality in the sense of Birkhoff (see also example 2.4).

\footnotetext{
This example is due to Ir. Jeroen Kuipers.
} 
Example 2.3 Let $m=4$. Let

$$
C=\left\{\left(\begin{array}{c}
1 \\
1 \\
-1 \\
-1
\end{array}\right),\left(\begin{array}{c}
1 \\
-1 \\
1 \\
-1
\end{array}\right),\left(\begin{array}{c}
1 \\
-1 \\
-1 \\
1
\end{array}\right),\left(\begin{array}{c}
1 / 5 \\
1 / 5 \\
1 / 5 \\
1 / 5
\end{array}\right)\right\}
$$

Let $\|x\| \equiv \max _{c \in C}|c \cdot x|$. It is easy to verify that $\|\cdot\|$ is indeed a norm on $\mathbf{R}^{4}$. Let

$$
X=\left\{\left(\begin{array}{l}
1 \\
0 \\
0 \\
0
\end{array}\right),\left(\begin{array}{l}
0 \\
1 \\
0 \\
0
\end{array}\right),\left(\begin{array}{l}
0 \\
0 \\
1 \\
0
\end{array}\right),\left(\begin{array}{l}
0 \\
0 \\
0 \\
1
\end{array}\right)\right\} .
$$

Then

$$
\left\|\left(\begin{array}{l}
1 \\
0 \\
0 \\
0
\end{array}\right)\right\|=1
$$

and

$$
\left\|\left(\begin{array}{l}
1 \\
0 \\
0 \\
0
\end{array}\right)+\left(\begin{array}{l}
0 \\
1 \\
0 \\
0
\end{array}\right)+\left(\begin{array}{l}
0 \\
0 \\
1 \\
0
\end{array}\right)+\left(\begin{array}{l}
0 \\
0 \\
0 \\
1
\end{array}\right)\right\|=\left\|\left(\begin{array}{l}
1 \\
1 \\
1 \\
1
\end{array}\right)\right\|=4 / 5
$$

So $X$ is not weakly $\|\cdot\|$-orthogonal.

On the other hand, we have that

$$
\begin{aligned}
\left\|\left(\begin{array}{l}
1 \\
0 \\
0 \\
0
\end{array}\right)+\lambda\left(\begin{array}{l}
0 \\
1 \\
0 \\
0
\end{array}\right)\right\| \geq \max \left\{\left(\begin{array}{c}
1 \\
1 \\
-1 \\
-1
\end{array}\right) \cdot\left(\begin{array}{l}
1 \\
\lambda \\
0 \\
0
\end{array}\right),\left(\begin{array}{c}
1 \\
-1 \\
1 \\
-1
\end{array}\right) \cdot\left(\begin{array}{l}
1 \\
\lambda \\
0 \\
0
\end{array}\right)\right\} \\
=\max \{1+\lambda, 1-\lambda\} \geq 1=\left\|\left(\begin{array}{l}
1 \\
0 \\
0 \\
0
\end{array}\right)\right\| .
\end{aligned}
$$

Therefore $\left(\begin{array}{l}1 \\ 0 \\ 0 \\ 0\end{array}\right)$ is $\|\cdot\|$-orthogonal to $\left(\begin{array}{l}0 \\ 1 \\ 0 \\ 0\end{array}\right)$ in the sense of Birkhoff. 
Similarly we can prove that each element of $X$ is $\|\cdot\|$-orthogonal to each other element in the sense of Birkhoff. Consequently, $X$ is $\|\cdot\|$-orthogonal in the sense of Birkhoff, but not weakly $\|\cdot\|$-orthogonal.

Lemma 2.3 gives an alternative characterization of $\| \cdot||$-orthogonality.

Lemma 2.3 Let $\|\cdot\|$ be a norm on $\mathbf{R}^{m}$. Let $X \subset \mathbf{R}^{m}, k=|X|$ and $x^{j} \in \mathbb{R}^{m}$ $(j=1, \ldots, k)$ with $X=\left\{x^{j}: j=1, \ldots, k\right\}$. Then the following assertions are equivalent:

(i) $X$ is $\|\cdot\|$-orthogonal.

(ii) For all $\lambda_{j}, \mu_{j} \in \mathbf{R}(j=1, \ldots, k)$ such that $0 \leq \mu_{j} \leq \lambda_{j}$ or $\lambda_{j} \leq \mu_{j} \leq 0$ for all $j \in\{1, \ldots, k\}$ we have $\left\|\sum_{j=1}^{k} \mu_{j} x^{j}\right\| \leq\left\|\sum_{j=1}^{k} \lambda_{j} x^{j}\right\|$.

Proof It is clear that (ii) implies (i) (choose $\mu_{l}=0$ and $\mu_{j}=\lambda_{j}$ for $j \neq l$ ). Conversely, assume $X$ is $\|\cdot\|$-orthogonal. Let $l \in\{1, \ldots, k\}$, and $\lambda_{j}, \mu_{j}$ as in (ii). It suffices to prove that $\left\|\sum_{\substack{j=1 \\ j \neq l}}^{k} \lambda_{j} x^{j}+\mu_{l} x^{l}\right\| \leq\left\|\sum_{j=1}^{k} \lambda_{j} x^{j}\right\|$.

If $\lambda_{l}=0$, then $\mu_{l}=0$ and hence $\left\|\sum_{\substack{j=1 \\ j \neq l}}^{k} \lambda_{j} x^{j}+\mu_{l} x^{l}\right\|=\left\|\sum_{j=1}^{k} \lambda_{j} x^{j}\right\|_{1}$. If $\lambda_{l} \neq 0$, then

$$
\begin{aligned}
\left\|\sum_{\substack{j=1 \\
j \neq l}}^{k} \lambda_{j} x^{j}+\mu_{l} x^{l}\right\| & =\left\|\sum_{\lambda_{l}}^{\mu_{l}} \sum_{j=1}^{k} \lambda_{j} x^{j}+\frac{\lambda_{l}-\mu_{l}}{\lambda_{l}} \sum_{\substack{j=1 \\
j \neq l}}^{k} \lambda_{j} x^{j}\right\| \\
& \leq\left|\frac{\mu_{1}}{\lambda_{l}}\right|\left\|\sum_{j=1}^{k} \lambda_{j} x^{j}\right\|+\left|\frac{\lambda_{l}-\mu_{l}}{\lambda_{l}}\right|\left\|\sum_{\substack{j=1 \\
j \neq l}}^{k} \lambda_{j} x^{j}\right\| \\
& \leq\left|\sum_{\lambda_{l}}^{\mu_{l}}\right|\left\|\sum_{j=1}^{k} \lambda_{j} x^{j}\right\|+\left|\frac{\lambda_{l}-\mu_{l}}{\lambda_{l}}\right|\left\|\sum_{j=1}^{k} \lambda_{j} x^{j}\right\| \\
& =\left\|\sum_{j=1}^{k} \lambda_{j} x^{j}\right\| .
\end{aligned}
$$

Like the usual concept of orthogonality (i.e., with respect to an inner product), $\|\cdot\|$-orthogonality and weak $\|\cdot\|$-orthogonality of a set imply the linear independence of the nonzero vectors in this set.

Lemma 2.4 Let $\|\cdot\|$ be a norm on $\mathbb{R}^{m}$. Let $X \subset \mathbf{R}^{m}$ be weakly $\|\cdot\|$ orthogonal. Then $X \backslash\{0\}$ is a linearly independent set.

Proof Suppose $X \backslash\{0\}$ is not linearly independent. Hence, there are $x \in$ $X \backslash\{0\}, y^{i} \in X \backslash\{x\}, \lambda_{i} \in \mathbf{R}, i=1, \ldots, k$ such that $x=\sum_{i=1}^{k} \lambda_{i} y^{i}$ for some 
$k \in\{1, \ldots, m\}$, which implies

$$
\left\|x+\sum_{i=1}^{k}\left(-\lambda_{i}\right) y^{i}\right\|=0<\|x\| .
$$

Consequently, $X$ is not weakly $\| \cdot \mid \cdot$-orthogonal.

This lemma has a straightforward consequence for the cardinality of a weakly orthogonal set:

Corollary 2.1 Let $\|\cdot\|$ be a norm on $\mathbf{R}^{m}$. Let $X \subset \mathbf{R}^{m}$ be weakly $\|\cdot\|$ orthogonal. Then the number of nonzero elements of $X$ does not exceed $m$.

Lemma 2.4 and corollary 2.1 are not valid if we replace "weakly $\| \cdot \mid$ orthogonal" by " $\|\cdot\|$-orthogonal in the sense of Birkhof". This is shown by the following example:

Example 2.4 Let $m=2$. Let $C=\left\{\left(\begin{array}{c}1 \\ \frac{1}{3} \sqrt{3}\end{array}\right),\left(\begin{array}{c}-1 \\ \frac{1}{3} \sqrt{3}\end{array}\right),\left(\begin{array}{c}0 \\ \frac{2}{3} \sqrt{3}\end{array}\right)\right\}$. Let $\|\cdot\|: \mathbb{R}^{2} \rightarrow \mathbf{R}$ be defined by $\|x\| \equiv \max _{c \in C}|c \cdot x|$. Then it is easy to verify that $\|\cdot\|$ is indeed a norm. (See figure 2.3.)

$$
\text { Let } X=\{u, v, w\} \text {, with } u=\left(\begin{array}{c}
1 \\
0
\end{array}\right), v=\left(\begin{array}{c}
\frac{1}{2} \\
\frac{1}{2} \sqrt{3}
\end{array}\right), w=\left(\begin{array}{c}
-\frac{1}{2} \\
\frac{1}{2} \sqrt{3}
\end{array}\right) \text {. }
$$

Then by some elementary calculations we can show that the unit ball of $\|\cdot\|$ (i.e., the set $\left\{x \in \mathbb{R}^{2}:\|x\| \leq 1\right\}$ ) is a hexagon with the origin as its centre and $u, v, w,-u,-v,-w$ as its vertices (so the unit ball corresponds to the shaded area in the picture).

Lemma $\mathrm{A} 1$ (see appendix A) implies that $X$ is $\|\cdot\|$-orthogonal in the sense of Birkhoff (note that $u=v-w, v=u+(-w), w=v-u$ ). On the other hand, since $m=2, X$ is a linearly dependent set. So a linearly dependent set which does not contain the zero vector may still be $\|\cdot\|$-orthogonal in the sense of Birkhoff. Similarly, a subset of $\mathbf{R}^{m}$ containing at least $m+1$ nonzero vectors, may still be $\|\cdot\|$-orthogonal in the sense of Birkhoff.

In view of lemma 2.4 , this example shows again that weak $\|\cdot\|$-orthogonality may be more restrictive than $\|\cdot\|$-orthogonality in the sense of Birkhoff.

Lemma 2.5 Let $\|\cdot\|$ be a norm on $\mathbf{R}^{m}$. Then there are $m$ different nonzero vectors $x^{i} \in \mathbb{R}^{m}$ such that $\left\{x^{j}: j=1, \ldots, m\right\}$ is weakly $\|\cdot\|$-orthogonal. 


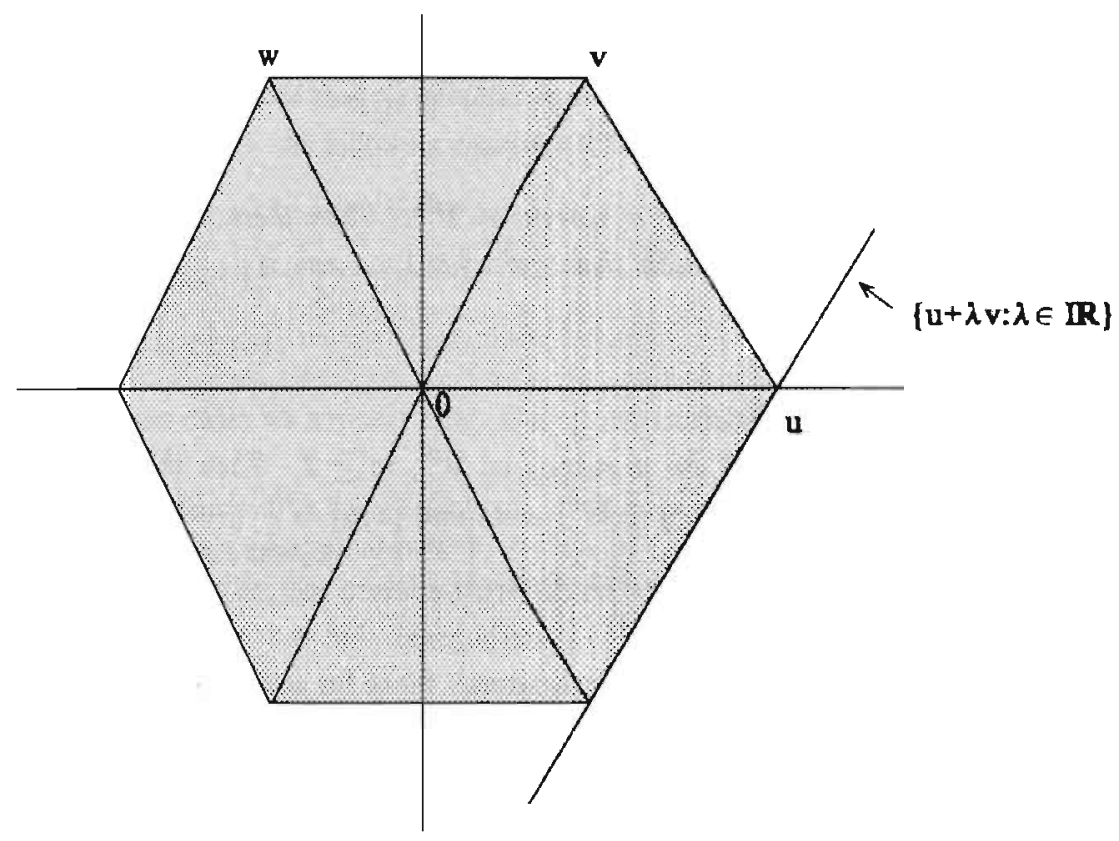

Figure 2.3: Example 2.4

Proof Let $C=\left\{x \in \mathbf{R}^{m}:\|x\| \leq 1\right\}$. Let $F: C \rightarrow \mathbf{R}: F\left(x^{1}, \ldots, x^{m}\right)=$ $\operatorname{det}\left(x^{1}, \ldots, x^{m}\right)$. Here $\operatorname{det}\left(x^{1}, \ldots, x^{m}\right)$ denotes the determinant of the matrix with $x^{1}, \ldots, x^{m}$ as column vectors.

Then $F$ is a continuous function. $C$ is a compact set and so is $C^{m}=$ $C \times C \times \ldots \times C$. Hence we can take $\bar{x}^{j} \in C(j=1, \ldots, m)$ such that $F\left(\bar{x}^{1}, \ldots, \bar{x}^{m}\right)$ is maximal over $C^{m}$.

Let $e^{j} \in \mathbb{R}^{m}(j=1, \ldots, m)$ be the $j$-th unit vector in $\mathbb{R}^{m}$. Let $\tau=$ $\frac{1}{\max _{j=1, \ldots, m}\left\|e^{j}\right\|}$. Then $\tau e^{j} \in C$ for $j=1, \ldots, m$.

Since $F\left(\tau e^{1}, \ldots, \tau e^{m}\right)=\tau^{m}>0$, we have that $F\left(\bar{x}^{1}, \ldots, \bar{x}^{m}\right) \geq \tau^{m}>0$. Therefore all $\bar{x}^{j}$ are nonzero and different.

Now suppose $\left\{\bar{x}^{j}: j=1, \ldots, m\right\}$ is not weakly $\|\cdot\|$-orthogonal. Then there are $l \in\{1, \ldots, m\}$ and $\lambda_{j} \in \mathbf{R}(j \in\{1, \ldots, m\})$ with $\left\|\lambda_{l} x^{l}\right\|>$ $\left\|\sum_{j=1}^{m} \lambda_{j} x^{j}\right\|$. W.l.o.g. assume that $\lambda_{l}=1$. So for some $\mu \in(0, \infty)$ we have $\left\|\sum_{j=1}^{m} \lambda_{j} \bar{x}^{j}+\mu \bar{x}^{l}\right\|=1$. Let $\tilde{x}^{k}=(1+\mu) \bar{x}^{k}+\lambda \bar{x}^{l}$ and $\tilde{x}^{j}=\bar{x}^{j}$ for $j=1, \ldots, m, j \neq k$. Then $\tilde{x}^{j} \in C$ for $j=1, \ldots, m$ and $F\left(\tilde{x}^{1}, \ldots, \tilde{x}^{m}\right)=$ $\operatorname{det}\left(\tilde{x}^{1}, \ldots, \tilde{x}^{m}\right)=(1+\mu) \operatorname{det}\left(\bar{x}^{1}, \ldots, \bar{x}^{m}\right)=(1+\mu) F\left(\bar{x}^{1}, \ldots, \bar{x}^{m}\right)>F\left(\bar{x}^{1}, \ldots\right.$, $\left.\bar{x}^{m}\right)$. This contradicts the maximality of $F$ over $C^{m}$. 
Consequently, $\left\{\bar{x}^{j}: j=1, \ldots, m\right\}$ is weakly $\|\cdot\|$-orthogonal.

Since weak $\| \cdot||$-orthogonality of a set implies $\|\cdot\|$ - orthogonality of that set in the sense of Birkhoff, we also have:

Corollary 2.2 Let $\|\cdot\|$ be a norm on $\mathbf{R}^{m}$. Then there are $m$ different nonzero vectors $x^{j} \in \mathbf{R}^{m}$ such that $\left\{x^{j}: j=1, \ldots, m\right\}$ is $\|\cdot\|$-orthogonal in the sense of Birkhoff.

Furthermore, we have:

Corollary 2.3 Let $\|\cdot\|$ be a norm on $\mathbf{R}^{m}, m \geq 2$. Then there are two different nonzero vectors $x, y \in \mathbb{R}^{m}$ such that $\{x, y\}$ is $\|\cdot\|$-orthogonal.

Proof By lemma 2.5 there are $m$ different nonzero vectors $x^{j} \in \mathbf{R}$ such that $\left\{x^{j}: j=1, \ldots, m\right\}$ is weakly $\|\cdot\|$-orthogonal. Let $x=x^{1}, y=x^{2}$. Then certainly $\{x, y\}$ is weakly $\|\cdot\|$-orthogonal. Since for sets with cardinality 2 $\|\cdot\|$-orthogonality is equivalent to weak $\|\cdot\|$-orthogonality, $\{x, y\}$ is $\|\cdot\|$ orthogonal.

For $m \geq 3$ an $\|\cdot\|$-orthogonal set with $m$ nonzero elements need not exist (this shows again in view of lemma 2.5 that $\|\cdot\|$-orthogonality may be more restrictive that weak $\|\cdot\|$-orthogonality), as is shown by the following example:

Example 2.5 Let $m=3$. Let

$$
C=\left\{\left(\begin{array}{l}
0 \\
1 \\
1
\end{array}\right),\left(\begin{array}{c}
\frac{3}{7} \\
\frac{4}{7} \\
1
\end{array}\right),\left(\begin{array}{c}
\frac{3}{7} \\
-\frac{4}{7} \\
1
\end{array}\right),\left(\begin{array}{c}
0 \\
-1 \\
1
\end{array}\right),\left(\begin{array}{c}
-\frac{4}{7} \\
-\frac{3}{7} \\
1
\end{array}\right),\left(\begin{array}{c}
-\frac{4}{7} \\
\frac{3}{7} \\
1
\end{array}\right)\right\} .
$$

Let $\|x\|=\max _{c \in C}\left|c^{T} x\right|$. It is easy to verify that $\|\cdot\|$ is indeed a norm on $\mathbf{R}^{3}$. Let $E=\left\{x \in \mathbf{R}^{3}:\|x\| \leq 1\right\}$. Then by some elementary (but laborious) arithmetical work, we can show that

$$
\begin{aligned}
E=\operatorname{conv} & \left\{\left(\begin{array}{c}
1 \\
1 \\
0
\end{array}\right),\left(\begin{array}{c}
1 \\
-1 \\
0
\end{array}\right),\left(\begin{array}{c}
-1 \\
1 \\
0
\end{array}\right),\left(\begin{array}{c}
-1 \\
-1 \\
0
\end{array}\right),\left(\begin{array}{l}
2 \\
0 \\
\frac{1}{7}
\end{array}\right),\right. \\
& \left.\left(\begin{array}{c}
-2 \\
0 \\
-\frac{1}{7}
\end{array}\right),\left(\begin{array}{c}
0 \\
0 \\
1
\end{array}\right),\left(\begin{array}{c}
0 \\
0 \\
-1
\end{array}\right)\right\} .
\end{aligned}
$$


Let $x^{1}, x^{2}, x^{3}$ be different nonzero vectors in $E$. Let $X=\left\{x^{1}, x^{2}, x^{3}\right\}$. We show that $X$ cannot be $\|\cdot\|$-orthogonal.

W.l.o.g. we assume that $\left\|x^{1}|=|\left|x^{2}\|=\| x^{3}\right| \mid=1\right.$, and $x_{3}^{1} \geq x_{3}^{2} \geq x_{3}^{3} \geq 0$. We will distinguish the following cases:

(i) $x_{3}^{1}=1$.

(ii) $x_{3}^{1}<1$ and $x_{3}^{2}>\frac{1}{7}$.

(iii) $x_{3}^{1}<1, x_{3}^{2} \leq \frac{1}{7}$.

For all these cases we suppose that $X$ is $\|\cdot\|$-orthogonal and derive a contradiction.

Case (i) In this case, $x^{1}=\left(\begin{array}{l}0 \\ 0 \\ 1\end{array}\right)$.

Also,

$$
\begin{aligned}
\left\|\left(\begin{array}{l}
1 \\
1 \\
0
\end{array}\right)+\lambda x^{1}\right\|= & \left\|\left(\begin{array}{c}
1 \\
1 \\
\lambda
\end{array}\right)\right\| \geq \max \left(\left(\frac{3}{7}, \frac{4}{7}, 1\right) \cdot\left(\begin{array}{c}
1 \\
1 \\
\lambda
\end{array}\right),\right. \\
& \left.\left|\left(-\frac{4}{7},-\frac{3}{7}, 1\right) \cdot\left(\begin{array}{c}
1 \\
1 \\
\lambda
\end{array}\right)\right|\right)=\max (1+\lambda, 1-\lambda)>1
\end{aligned}
$$

for all $\lambda \in \mathbb{R} \backslash\{0\}$. By lemma 2.4 there are $\lambda_{1}, \lambda_{2}, \lambda_{3} \in \mathbf{R}$ such that $\left(\begin{array}{l}1 \\ 1 \\ 0\end{array}\right)=$ $\lambda_{1} x^{1}+\lambda_{2} x^{2}+\lambda_{3} x^{3}$. Hence, by the $\|\cdot\|$-orthogonality of $X,\left\|\lambda_{2} x^{2}+\lambda_{3} x^{3}\right\| \leq$ $\left\|\lambda_{1} x^{1}+\lambda_{2} x^{2}+\lambda_{3} x^{3}\right\|$. So, $\left\|\left(\begin{array}{l}1 \\ 1 \\ 0\end{array}\right)-\lambda_{1} x^{1}\right\| \leq 1$. Hence, $\lambda_{1}=0$. Therefore $\left(\begin{array}{l}1 \\ 1 \\ 0\end{array}\right) \in \operatorname{aff}\left\{x^{2}, x^{3}\right\}$

Similarly, $\left(\begin{array}{c}1 \\ -1 \\ 0\end{array}\right) \in \operatorname{aff}\left\{x^{2}, x^{3}\right\}$ and $\left(\begin{array}{c}2 \\ 0 \\ \frac{1}{7}\end{array}\right) \in$ aff $\left\{x^{2}, x^{3}\right\}$.

Since $\left(\begin{array}{c}1 \\ 1 \\ 0\end{array}\right),\left(\begin{array}{c}1 \\ -1 \\ 0\end{array}\right)$ and $\left(\begin{array}{c}2 \\ 0 \\ \frac{1}{7}\end{array}\right)$ are linearly independent, this implies a 
contradiction.

Cases (ii) and (iii) Let $S=\left\{x \in E:\|x\|=1,-\frac{1}{7}<x_{3}<\frac{1}{7}\right\}$. By lemma 2.4 there are $\mu_{1}, \mu_{2}, \mu_{3} \in \mathbf{R}$ such that $\left(\begin{array}{l}0 \\ 0 \\ 1\end{array}\right)=\mu_{1} x^{1}+\mu_{2} x^{2}+\mu_{3} x^{3}$. By the $\| \cdot \mid$-orthogonality of $X$,

$$
\begin{gathered}
\left\|\mu_{1} x^{1}+\mu_{2} x^{2}+\mu_{3} x^{3}\right\| \geq \max _{i=1,2,3}\left|\mu_{i}\right| \| \frac{\mu_{1}}{\max _{i=1,2,3}\left|\mu_{i}\right|} x^{1}+ \\
\frac{\mu_{2}}{\max _{i=1,2,3}\left|\mu_{i}\right|} x^{2}+\frac{\mu_{3}}{\max _{i=1,2,3}\left|\mu_{i}\right|} x^{3} \| \geq \max _{i=1,2,3}\left|\mu_{i}\right| .
\end{gathered}
$$

So $\left|\mu_{i}\right| \leq 1$ for $i=1,2,3$.

Let $l \in\{1,2,3\}$. Suppose $x_{3}^{l}>\frac{1}{7}$. Since $\left\|x^{l}\right\|=1$, there is a $b \in S$ such that $x^{l} \in\left(b,\left(\begin{array}{l}0 \\ 0 \\ 1\end{array}\right)\right)$. Hence, $b=x^{l}+\tau\left(x^{l}-\left(\begin{array}{l}0 \\ 0 \\ 1\end{array}\right)\right)$ for some $\tau>0$.

So $1=\|b\|=\left\|x^{l}-\tau\left(x^{l}-\mu_{1} x^{1}-\mu_{2} x^{2}-\mu_{3} x^{3}\right)\right\|=\|\left(1+\tau\left(1-\mu_{l}\right)\right) x^{l}+$ $\tau \sum_{i \neq l} \mu_{i} x^{i}|| \geq\left|1+\tau\left(1-\mu_{l}\right)\right|$. Hence, $\mu_{l}=1$. Consequently,

$$
\forall l \in\{1,2,3\}: \mu_{l}=1 \text { if } x_{3}^{l}>\frac{1}{7} .
$$

Case (ii) In this case $x_{3}^{1} \geq x_{3}^{2}>\frac{1}{7}$. So by (2.1), $\left(\begin{array}{l}0 \\ 0 \\ 1\end{array}\right)=x^{1}+x^{2}+\mu_{3} x^{3}$. By the $\|\cdot\|$-orthogonality of $X, 1=\left\|x^{1}\right\| \leq\left\|x^{1}+x^{2}\right\| \leq\left\|x^{1}+x^{2}+\mu_{3} x^{3}\right\|=1$. Also, $x_{3}^{1}+x_{3}^{2} \geq \frac{2}{7}$. Hence $x^{1}+x^{2} \in\left(\left(\begin{array}{l}1 \\ 0 \\ 0\end{array}\right), c\right)$ for some $c \in S$.

So $c=x^{1}+x^{2}+\tau\left(x^{1}+x^{2}-\left(x^{1}+x^{2}+\mu_{3} x^{3}\right)\right)=x^{1}+x^{2}-\tau \mu_{3} x^{3}$ for some $\tau \in \mathbb{R}$. Since ||$c \|=1,\left|\tau \mu_{3}\right| \leq 1$. Hence $\frac{1}{7} \geq c_{3}=x_{3}^{1}+x_{3}^{2}-\tau \mu_{3} x_{3}^{3} \geq$ $x_{3}^{1}+x_{3}^{2}-x_{3}^{3} \geq x_{3}^{1}>\frac{1}{7}$, which is a contradiction.

Case (iii) In this case $x_{3}^{3} \leq x_{3}^{2} \leq \frac{1}{7}$.

So, $1=\mu_{1} x_{3}^{1}+\mu_{2} x_{3}^{2}+\mu_{3} x_{3}^{3} \leq x_{3}^{1}+\frac{1}{7}+\frac{1}{7}$. Hence, $x_{3}^{1} \geq \frac{5}{7}$. So by $(2.1)$, $\left(\begin{array}{l}0 \\ 0 \\ 1\end{array}\right)=x^{1}+\mu_{2} x^{2}+\mu_{3} x^{3}$. Hence, $x^{1} \in\left(\left(\begin{array}{l}1 \\ 0 \\ 0\end{array}\right), d\right)$ for some $d \in S$. So $d=x^{1}+\tau\left(x^{1}-\left(x^{1}+\mu_{2} x^{2}+\mu_{3} x^{3}\right)\right)=x^{1}-\tau \mu_{2} x^{2}-\tau \mu_{3} x^{3}$ for some $\tau \in \mathbb{R}$. 
Since ||$d||=1,\left|\tau \mu_{2}\right| \leq 1$ and $\left|\tau \mu_{3}\right| \leq 1$. So $\frac{1}{7} \geq d_{3}=x_{3}^{1}-\tau \mu_{2} x_{3}^{2}-\tau \mu_{3} x_{3}^{3} \geq$ $x_{3}^{1}-x_{3}^{2}-x_{3}^{3} \geq \frac{5}{7}-\frac{1}{7}-\frac{1}{7} \geq \frac{3}{7}$ which is a contradiction.

The norm in the above example is quite artificial and we cannot imagine an application of our model where this norm would be suitable. For norms mostly used (e.g. p-norms) an $\|\cdot\|$-orthonormal set with cardinality $m$ is easily obtained by taking the standard basis of unit vectors.

The same is true for norms which are induced by a real inner product in view of lemma 2.3. As, in this case, $\|\cdot\|$-orthogonality is equivalent to the standard definition, there are infinitely many $\|\cdot\|$-orthonormal sets with cardinality $m$.

In the case of strictly convex norms, the introduced orthogonality concepts can be characterized in a stronger way, as is shown by the next three lemmas.

Lemma 2.6 Let $\|\cdot\|$ be a strictly convex norm on $\mathbf{R}^{m}$. Let $X \subset \mathbb{R}^{m}$, $k=|X|$ and $x^{j} \in \mathbb{R}^{m} \backslash\{0\}(j=1, \ldots, k\}$ with $X=\left\{x^{j}: j=1, \ldots, k\right\}$. Then the following assertions are equivalent:

(i) $X$ is $\|\cdot\|$-orthogonal.

(ii) For all $l \in\{1, \ldots, k\}$, and all $\lambda_{j} \in \mathbf{R}(j=1, \ldots, k)$ with $\lambda_{l} \neq 0$ we have that

$$
\left\|\sum_{\substack{j=1 \\ j \neq 1}}^{k} \lambda_{j} x^{j}\right\|<\left\|\sum_{j=1}^{k} \lambda_{j} x^{j}\right\| .
$$

(iii) For all $\lambda_{j}, \mu_{j} \in \mathbf{R}(j=1, \ldots, k)$ with $0 \leq \mu_{j} \leq \lambda_{j}$ or $\lambda_{j} \leq \mu_{j} \leq 0$ for $j=1, \ldots, k$ and $\lambda_{l} \neq \mu_{l}$ for some $l \in\{1, \ldots, k\}$ we have that

$$
\left\|\sum_{j=1}^{k} \mu_{j} x^{j}\right\|<\left\|\sum_{j=1}^{k} \lambda_{j} x^{j}\right\| .
$$

Proof (i) $\Rightarrow$ (iii) Let $\left\{x^{j}: j=1, \ldots, k\right\}$ be $\|\cdot\|$-orthogonal. Suppose there are $l \in\{1, \ldots, k\}$ and $\lambda_{j}, \mu_{j} \in \mathbf{R}(j=1, \ldots, k)$ with $0 \leq \mu_{j} \leq \lambda_{j}$ or $\lambda_{j} \leq \mu_{j} \leq 0$ for all $j=1, \ldots, k$ and $\lambda_{l} \neq \mu_{l}$ such that $\left\|\sum_{j=1}^{k} \lambda_{j} x^{j}\right\| \leq$ $\left\|\sum_{j=1}^{k} \mu_{j} x^{j}\right\|$.

Let $\alpha_{j}=\frac{1}{2} \lambda_{j}+\frac{1}{2} \mu_{j}$ for $j=1, \ldots, k$. Then by lemma 2.1 (iii) we have that

$$
\left\|\sum_{j=1}^{k} \alpha_{j} x^{j}\right\|<\left\|\sum_{j=1}^{k} \mu_{j} x^{j}\right\| .
$$


Since $0 \leq \mu_{j} \leq \alpha_{j}$ or $\alpha_{j} \leq \mu_{j} \leq 0$ for $j=1, \ldots, k$ we have by the $\|\cdot\|$ orthogonality of $\left\{x^{j}: j=1, \ldots, k\right\}$ and lemma 2.3 that $\left\|\sum_{j=1}^{k} \mu_{j} x^{j}\right\| \leq$ $\left\|\sum_{j=1}^{k} \alpha_{j} x^{j}\right\|$. This contradicts (2.2).

(iii) $\Rightarrow$ (ii) Assume (ii). Let $l \in\{1, \ldots, k\}$ and $\lambda_{j} \in \mathbb{R}(j=1, \ldots, k)$ with $\lambda_{l} \neq 0$. Let $\mu_{l}=0$ and $\mu_{j}=\lambda_{j}$ for all $j \in\{1, \ldots, k\} \backslash\{l\}$. Then by (ii),

$$
\left\|\sum_{\substack{j=1 \\ j \neq l}}^{k} \lambda_{j} x^{j}\right\|=\left\|\sum_{j=1}^{k} \mu_{j} x^{j}\right\|<\left\|\sum_{j=1}^{k} \lambda_{j} x^{j}\right\| .
$$

(ii) $\Rightarrow$ (i) Trivial.

Similarly, we have:

Lemma 2.7 Let $\|\cdot\|$ be a strictly convex norm on $\mathbf{R}^{m}$. Let $X \subset \mathbf{R}^{m}$, $k:=|X|$ and $x^{j} \in \mathbb{R}^{m} \backslash\{0\}(j=1, \ldots, k)$ with $X=\left\{x^{j}: j=1, \ldots, k\right\}$. Then the following assertions are equivalent:

(i) $X$ is weakly $\|\cdot\|$-orthogonal.

(ii) For all $l \in\{1, \ldots, k\}$, and all $\lambda_{j} \in \mathbf{R}(j=1, \ldots, k)$ with $\lambda_{i} \neq 0$ for some $i \in\{1, \ldots, k\} \backslash\{l\}$ we have that $\left\|\lambda_{l} x^{l}\right\|<\left\|\sum_{j=1}^{k} \lambda_{j} x^{j}\right\|$.

Proof (i) $\Rightarrow$ (ii) Let $\left\{x^{j}: j=1, \ldots, k\right\}$ be weakly $\|\cdot\|$-orthogonal. Suppose there are $l \in\{1, \ldots, k\}, i \in\{1, \ldots, k\} \backslash\{l\}$ and $\lambda_{j} \in \mathbb{R}(j=1, \ldots, k)$ with $\lambda_{i} \neq 0$ and $\left\|\sum_{j=1}^{k} \lambda_{j} x^{j}\right\| \leq\left\|\lambda_{l} x^{l}\right\|$. Let $\mu_{l}=\lambda_{l}$, and $\mu_{j}=\frac{1}{2} \lambda_{j}$ for all $j \in$ $\{1, \ldots, k\} \backslash\{l\}$. Then by lemma 2.1 (iii), $\left\|\sum_{j=1}^{k} \mu_{j} x^{j}\right\|<\left\|\lambda_{l} x^{l}\right\|=\left\|\mu_{l} x^{l}\right\|$. This contradicts the weak $\|\cdot\|$-orthogonality of $X$.

(ii) $\Rightarrow$ (i) Trivial.

And also:

Lemma 2.8 Let $\|\cdot\|$ be a strictly convex norm on $\mathbb{R}^{m}$. Let $x, y \in \mathbb{R}^{m}$. Then the following assertions are equivalent:

(i) $x$ is $\|\cdot\|$-orthogonal to $y$ in the sense of Birkhoff.

(ii) For all $\lambda \in \mathbb{R}\{0\}$ we have $\|x\|<\|x+\lambda y\|$. 
Proof (i) $\Rightarrow$ (ii) Assume $x$ is $\|\cdot\|$-orthogonal to $y$ in the sense of Birkhoff. Let $\lambda \in \mathbf{R} \backslash\{0\}$. Suppose $\|x+\lambda y\| \leq\|x\|$. Then by lemma 2.2 (iii), $\left\|x+\frac{\lambda}{2} y\right\|<\|x\|$. So $x$ is not $\|\cdot\|$-orthogonal to $y$ in the sense of Birkhoff. This contradicts our assumption.

Consequently, we conclude with respect to sets:

Corollary 2.4 Let $\|\cdot\|$ be a strictly convex norm on $\mathbb{R}^{m}$. Let $X \subset \mathbf{R}^{m}$. Then the following assertions are equivalent:

(i) $X$ is $\|\cdot\|$-orthogonal in the sense of Birkhoff.

(ii) For all different nonzero $x, y \in X$ and all $\lambda \in \mathbb{R} \backslash\{0\}$ we have $\|x\|<$ $\|x+\lambda y\|$.

\subsection{Pareto optimality and strategy-proofness}

In this section we discuss how a metric or norm on $\mathbf{R}^{m}$ induces a class of preferences on the set of alternatives $A \subset \mathbb{R}^{m}$. Pareto optimality and strategy-proofness with respect to such a class can be reformulated in terms of the metric or norm by which that class is induced. Several implications of Pareto optimality or strategy-proofness with respect to a (strictly convex) norm are studied. At the end of the section we will investigate under what circumstances coordinatewise median schemes satisfy Pareto optimality or strategy-proofness with respect to a strictly convex norm.

Let $A \subset \mathbb{R}^{m}$. Let $\delta$ be a metric ${ }^{5}$ on $A$. We call a preference $R$ on $A$ induced by $\delta$ if there is a $z \in A$ such that for all $x, y \in A$ we have that $(x, y) \in R$ if and only if $\delta(x, z) \leq \delta(y, z)$. So for such a preference there is one best point $z$ in $A$, and $x \in A$ is (weakly) preferred to $y \in A$ if $x$ is at least as close to $z$ (measured according to metric $\delta$ ) as $y$ is. We call a class of preferences $P$ induced by $\delta$ if all $R \in P$ are induced by $\delta$ and $\cup_{R \in} \operatorname{pbest}(R)=A$.

From these definitions it is easy to verify that every class of preferences which is induced by a metric is standard (see section 1.2). Hence, the lemmas 1.1-1.4 apply. It is also obvious that each metric on $A$ uniquely determines a class of preferences on $A$.

If a metric $\delta$ on $A$ is induced by a norm $\|\cdot\|$ on $\mathbf{R}^{m}$, (i.e., $\delta(x, y)=\|x-y\|$ for all $x, y \in A$ ) we call a preference $R$ (class of preferences $P$ ) induced by the norm $\|\cdot\|$ if $R(P)$ is induced by $\delta$.

\footnotetext{
${ }^{5}$ For the definition of a metric, see appendix $\mathrm{A}$
} 
Let $\varphi: A^{N} \rightarrow A$, and let $\delta$ be a metric on $A$. We say that $\varphi$ satisfies a property $\mathrm{P}$ with respect to $\delta$, if $\varphi$ satisfies $\mathrm{P}$ with respect to the class of preferences induced by $\delta$. Similarly, we say that $\varphi$ satisfies a property $P$ with respect to a norm $\|\cdot\|$ on $\mathbf{R}^{m}$, if $\varphi$ satisfies $P$ with respect to the class of preferences induced by $\|\cdot\|$.

Thus, several versions of Pareto optimality and strategy-proofness with respect to a metric $\delta$ on $A$ now can be reformulated as follows:

PO: $\varphi$ is Pareto optimal with respect to $\delta$, if for no $p \in A^{N}$ there is an $x \in A$ with $\delta(x, p(i)) \leq \delta(\varphi(p), p(i))$ for all $i \in N$ and at least one of these inequalities strict.

WPO: $\varphi$ is weakly Pareto optimal with respect to $\delta$, if for no $p \in A^{N}$ there is an $x \in A$ with $\delta(x, p(i))<\delta(\varphi(p), p(i))$.

SPO: $\varphi$ is strongly Pareto optimal with respect to $\delta$, if for no $p \in A^{N}$ there is an $x \in A \backslash\{\varphi(p)\}$ with $\delta(x, p(i)) \leq \delta(\varphi(p), p(i))$.

SP: $\varphi$ is strategy-proof with respect to $\delta$, if for all $i \in N$ and all profiles $p, q \in A^{N}$ with $p={ }_{N \backslash\{i\}} q$ we have:

$$
\delta(p(i), \varphi(p)) \leq \delta(p(i), \varphi(q)) .
$$

ISP: $\varphi$ is intermediate strategy-proof with respect to $\delta$, if for all $S \subset N$, $x \in A^{N}$, and $p, q \in A$ with $p(S)=\{x\}$ and $p={ }_{N \backslash S} q$ we have: $\delta(x, \varphi(p)) \leq$ $\delta(x, \varphi(q))$.

CSP: $\varphi$ is coalitional strategy-proof with respect to $\delta$, if for all $S \subset N$, $p, q \in A^{N}$ with $p=N \backslash S q$ and $\delta(p(i), \varphi(q)) \leq \delta(p(i), \varphi(p))$ for all $i \in S$ we have: $\delta(p(i), \varphi(p)) \leq \delta(p(i), \varphi(q))$ for some $i \in S$.

In the remainder of this section we restrict ourselves to normed spaces. We will investigate some implications of Pareto optimality and strategyproofness, especially when a strictly convex norm is involved.

Proposition 2.1 Let $\|\cdot\|$ be a strictly convex norm on $\mathbb{R}^{m}$. Let $A$ be a nonempty open subset of $\mathbf{R}^{m}$. Then with respect to $\|\cdot\|, P O, S P O$ and WPO are equivalent.

Proof It is obvious that SPO implies PO and PO implies WPO, so it suffices to prove that WPO implies SPO. 
Assume $\varphi: A^{N} \rightarrow A$ satisfies WPO. Suppose $\varphi$ does not satisfy SPO. Then we can take $p \in A^{N}, x \in A \backslash\{\varphi(p)\}$ such that $\|x-p(i)\| \leq\|\varphi(p)-p(i)\|$ for all $i \in N$. By the openness of $A$ we can take $y \in(\varphi(p), x)$ with $y \in A$. Hence lemma 2.1 (iii) implies that $\|y-p(i)\|<\|\varphi(p)-p(i)\|$ for all $i \in N$. This contradicts WPO. Consequently, WPO implies SPO.

Proposition 2.1 does not need to hold for norms which are not strictly convex, as is demonstrated by the following example. In example 2.6 we consider a norm which is not strictly convex. We show that with respect to this norm, PO is more restrictive than WPO.

Example 2.6 Let $\|\cdot\|_{\infty}$ be the maximum norm on $\mathbb{R}^{2}$, i.e. $\|x\|_{\infty} \equiv$ $\max \left\{\left|x_{1}\right|,\left|x_{2}\right|\right\}$. Let $A=\mathbb{R}^{2}$. Let $\varphi: A^{3} \rightarrow A$ be the mean scheme, i.e. $\varphi(p)=\frac{1}{3} p(1)+\frac{1}{3} p(2)+\frac{1}{3} p(3)$ for all $p \in A^{3}$. Let $q(1)=\left(\begin{array}{l}0 \\ 0\end{array}\right), q(2)=$ $\left(\begin{array}{l}2 \\ 0\end{array}\right), q(3)=\left(\begin{array}{l}1 \\ 1\end{array}\right)$

Then $\varphi(q)=\left(\begin{array}{c}1 \\ \frac{1}{3}\end{array}\right)$. Hence, $\left\|\left(\begin{array}{l}1 \\ 1\end{array}\right)-\left(\begin{array}{l}0 \\ 0\end{array}\right)\right\|_{\infty} \leq\left\|\varphi(p)-\left(\begin{array}{l}0 \\ 0\end{array}\right)\right\|_{\infty}$, $\left\|\left(\begin{array}{l}1 \\ 1\end{array}\right)-\left(\begin{array}{l}2 \\ 0\end{array}\right)\right\|_{\infty} \leq\left\|\varphi(p)-\left(\begin{array}{l}0 \\ 0\end{array}\right)\right\|_{\infty}$ and $\left\|\left(\begin{array}{l}1 \\ 1\end{array}\right)-\left(\begin{array}{l}1 \\ 1\end{array}\right)\right\|_{\infty}<$ $\left\|\varphi(p)-\left(\begin{array}{l}1 \\ 1\end{array}\right)\right\|_{\infty}$.

So $\varphi$ is not Pareto optimal with respect to $\|\cdot\|_{\infty}$. Now suppose $\varphi$ does not satisfy WPO with respect to $\|\cdot\|_{\infty}$. Then there are $p \in A^{3}$, $x \in A$ such that $\|x-p(i)\|_{\infty}<\|\varphi(p)-p(i)\|_{\infty}$ for $i=1,2,3$. If the $p(i)$ are collinear there are $j, k \in\{1,2,3\}$ such that $\varphi(p) \in[p(j), p(k)]$, and such an $x$ cannot exist. Now assume the $p(i)$ are noncollinear. Since $\varphi(p)$ is the center of gravity of the $p(i), \varphi(p) \in \operatorname{conv}\{p(1), p(2), p(3)\}$ and $\varphi(p) \notin[p(1), p(2)] \cup[p(2) \cup p(3)] \cup[p(1), p(3)]$. Hence, there are $j, k \in\{1,2,3\}$ such that $[p(k), p(l)] \cap[x, \varphi(p), \rightarrow) \backslash[x, \varphi(p)] \neq \emptyset$, say it contains the point $y$. So $\varphi(p) \in(x, y)$. Hence, because of the triangular inequality for norms,

$$
\begin{gathered}
\|y-p(k)\|_{\infty}+\|y-p(l)\|_{\infty}=\|p(k)-p(l)\|_{\infty} \\
\leq\|\varphi(p)-p(k)\|_{\infty}+\|\varphi(p)-p(l)\|_{\infty} .
\end{gathered}
$$

So $\|y-p(k)\|_{\infty} \leq\|\varphi(p)-p(k)\|_{\infty}$ or $\|y-p(l)\|_{\infty} \leq\|\varphi(p)-p(l)\|_{\infty}$. W.l.o.g. assume that $\|y-p(k)\|_{\infty} \leq\|\varphi(p)-p(k)\|_{\infty}$. We know that $\|x-p(k)\|_{\infty}<$ $\|\varphi(p)-p(k)\|_{\infty}$. Let $\lambda \in(0,1)$ be such that $\varphi(p)=\lambda x+(1-\lambda) y$. Then 
$\|\varphi(p)-p(k)\|_{\infty}=\|\lambda(x-p(k))+(1-\lambda)(y-p(k))\|_{\infty} \leq \lambda\|x-p(k)\|_{\infty}+(1-$ $\lambda)\|y-p(k)\|_{\infty}<\lambda\|\varphi(p)-p(k)\|_{\infty}+(1-\lambda)\|\varphi(p)-p(k)\|_{\infty}=\|\varphi(p)-p(k)\|_{\infty}$, a contradiction.

Consequently, $\varphi$ satisfies WPO with respect to $\|\cdot\|_{\infty}$.

Example 2.7 demonstrates that there are at least some norms $\|\cdot\|$ such that SPO with respect to $\|\cdot\|$ is more restrictive than PO with respect to $\|\cdot\|$.

Example 2.7 Let $\|\cdot\|_{1}$ be the sumnorm on $\mathbb{R}^{2}$, i.e., $\|x\|_{1} \equiv\left|x_{1}\right|+\left|x_{2}\right|$. Let $A=\mathbb{R}^{2}$. Let $\varphi: A^{2} \rightarrow A$ be the standard median scheme with one constant point $c^{1}=\left(\begin{array}{c}-\infty \\ -\infty\end{array}\right)$. Then $\varphi(x, y) \equiv\left(\begin{array}{c}\min \left(x_{1}, y_{1}\right) \\ \min \left(x_{2}, y_{2}\right)\end{array}\right)$.

Hence, $\|x-\varphi(x, y)\|_{1}+\|\varphi(x, y)-y\|_{1} \equiv\|x-y\|_{1}$. This implies that $\varphi$ satisfies PO with respect to $\|\cdot\|_{1}$ (proof left to the reader).

On the other hand, let $u=\left(\begin{array}{l}1 \\ 0\end{array}\right), v=\left(\begin{array}{l}0 \\ 1\end{array}\right)$. Then $\varphi(u, v)=\left(\begin{array}{l}0 \\ 0\end{array}\right)$. Hence $\left\|u-\left(\begin{array}{l}1 \\ 1\end{array}\right)\right\|_{1} \leq\|u-\varphi(u, v)\|_{1}$ and $\left\|v-\left(\begin{array}{l}1 \\ 1\end{array}\right)\right\|_{1} \leq\|v-\varphi(u, v)\|_{1}$. This shows that $\varphi$ does not satisfy SPO with respect to $\|\cdot\|_{1}$. In fact it can be demonstrated that SPO with respect to $\|\cdot\|_{1}$ implies that $\varphi(x, y) \in\{x, y\}$ for all $x, y \in A$ with $x_{1} \neq x_{2}$ and $y_{1} \neq y_{2}$.

Conjecture 2.1 Let $n \geq 3$. Let $\|\cdot\|$ be a norm on $\mathbb{R}^{m}$ which is not strictly convex. Let $A$ be a nonempty open subset of $\mathbf{R}^{m}$. Then:

(i) there are voting schemes $\varphi: A^{N} \rightarrow A$ such that $\varphi$ satisfies WPO with respect to $\|\cdot\|$ and $\varphi$ does not satisfy $P O$ with respect to $\|\cdot\|$.

(ii) there are voting schemes $\varphi: A^{N} \rightarrow A$ such that $\varphi$ satisfies $P O$ with respect to $\|\cdot\|$ and $\varphi$ does not satisfy SPO with respect to $\|\cdot\|$.

If $\|\cdot\|$ is not strictly convex, the implications of PO, WPO or SPO seem to be very irregular. There are several papers in which these implications are studied. We mention Chalmet (1983), and Ward and Wendell (1983).

As we are (in the framework of this thesis) mainly focussed on norms which are strictly convex, we leave it to the reader to confirm or refute this conjecture.

Lemma 2.9 Let $A$ be a nonempty open subset of $\mathbb{R}^{2}$. Let $\|\cdot\|$ be a norm on $\mathbb{R}^{2}$. Let $\varphi: A^{N} \rightarrow A$ satisfy $S P O$ with respect to $\|\cdot\|$. Then $\varphi(p) \in$ $\operatorname{conv}\{p(i): i \in N\}$ for all $p \in A^{N}$. 
Proof Assume $\varphi$ satisfies SPO with respect to $\|\cdot\|$. Suppose there is a $p \in A^{N}$ such that $\varphi(p) \notin \operatorname{conv}\{p(i): i \in N\}$ (cf. Figure 2.4). Let - denote the standard inner product. Because of a well-known separation theorem from convex analysis (see Rockafellar, 1970, theorem 11.4) there are $\tau \in \mathbf{R}$, $c \in \mathbb{R}^{2}$ such that $c \cdot \varphi(p)>\tau$ and $c \cdot x<\tau$ for all $x \in \operatorname{conv}\{p(1), \ldots, p(n)\}$. Let $q(i)=\mu_{i} \varphi(p)+\left(1-\mu_{i}\right) p(i)$, with $\mu_{i}=\frac{r-c \cdot p(i)}{c \cdot \varphi(p)-c \cdot p(i)}(i \in N)$. Hence, $c \cdot q(i)=\tau$ for all $i \in N$. So the $q(i)$ are collinear. Let $k, l \in N$ be such that $q(i) \in[q(k), q(l)]$ for all $i \in N$. Because of the triangular inequality for norms we have that

$$
\|\varphi(p)-q(k)\|+\|\varphi(p)-q(l)\| \geq\|q(k)-q(l)\|
$$

Let

$$
a=\frac{\|\varphi(p)-q(l)\| q(k)+\|\varphi(p)-q(k)\| q(l)}{\|\varphi(p)-q(b)\|+\|\varphi(p)-q(l)\|} .
$$

Then by (2.3) we obtain

$$
\|a-q(k)\|=\frac{\|\varphi(p)-q(k)\|\|\varphi(k)-q(l)\|}{\|\varphi(p)-q(l)\|+\|\varphi(p)-q(k)\|} \leq\|\varphi(p)-q(k)\| .
$$

Similarly $\|a-q(l)\| \leq\|\varphi(p)-q(l)\|$. If $q(i) \in[q(k), a]$, then $\|a-q(i)\|=$ $\|a-q(k)\|-\|q(k)-q(i)\| \leq\|\varphi(p)-q(k)\|-\|q(k)-q(i)\| \leq\|\varphi(p)-q(i)\|$. Similarly, $\|a-q(i)\| \leq\|\varphi(p)-q(i)\|$ if $q(i) \in[q(l), a]$. Hence, $\|a-q(i)\| \leq$ $\|\varphi(p)-q(i)\|$ for all $i \in N$. Therefore, $\|a-p(i)\| \leq\|a-q(i)\|+\|q(i)-p(i)\| \leq$ $\|\varphi(p)-q(i)\|+\|q(i)-p(i)\|=\|\varphi(p)-p(i)\|$ for all $i \in N$. By the openness of $A$, we can choose $b \in(a, \varphi(p))$ with $b \in A$. Then lemma $A 1$ (ii) (see appendix A) implies that $\|b-p(i)\| \leq\|\varphi(p)-p(i)\|$ for all $i \in N$. Consequently, $\varphi$ is not strongly Pareto optimal with respect to $\|\cdot\|$.

Lemma 2.10 Let $A$ be a nonempty open subset of $\mathbf{R}^{2}$. Let $\|\cdot\|$ be a norm on $\mathbb{R}^{2}$. Let $\varphi: A^{N} \rightarrow A$ be such that $\varphi(p) \in \operatorname{conv}\{p(i): i \in N\}$ for all $p \in A^{N}$. Then $\varphi$ satisfies WPO with respect to $\|\cdot\|$.

Proof Assume

$$
\varphi(p) \in \operatorname{conv}\{p(1), \ldots, p(n)\} \text { for all } p \in A^{N} .
$$

Let $\tilde{p} \in A^{N}, y \in A$. It suffices to prove that $\|\varphi(\tilde{p})-\tilde{p}(i)\| \leq\|y-\tilde{p}(i)\|$ for at least one $i \in N$. By (2.4) we can take $j, k \in N$ and $z \in[\tilde{p}(j), \tilde{p}(k)]$ such that $\varphi(\tilde{p}) \in[z, y]$. Then by the triangular inequality for norms,

$$
\|y-\tilde{p}(j)\|+\|y-\tilde{p}(k)\| \geq\|\tilde{p}(j)-\tilde{p}(k)\|=\|z-\tilde{p}(j)\|+\|z-\tilde{p}(k)\| .
$$




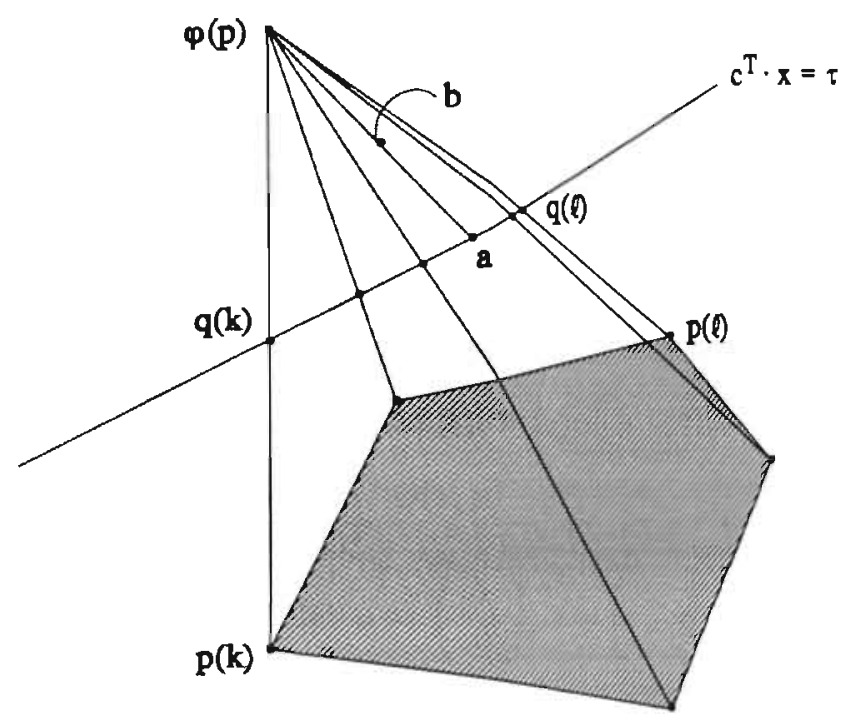

Figure 2.4: Proof of Lemma 2.9

Assume w.l.o.g. that $\|\tilde{p}(j)-y\| \geq\|\tilde{p}(j)-z\|$ (otherwise interchange the roles of $y$ and $z$ ). Since $\varphi(\tilde{p})-\tilde{p}(j) \in[z-\tilde{p}(j), y-\tilde{p}(j)]$ this implies by lemma A1 (ii) (see appendix A), that $\|\varphi(\tilde{p})-\tilde{p}(j)\| \leq\|y-\tilde{p}(j)\|$.

The following theorem is a direct consequence of lemmas 2.9, 2.10 and proposition 2.1.

Theorem 2.1 Let $A$ be a nonempty open subset of $\mathbf{R}^{2}$. Let $\|\cdot\|$ be a strictly convex norm on $\mathbf{R}^{2}$. Let $\varphi: A^{N} \rightarrow A$ be a voting scheme. Then the following assertions are equivalent:

(i) $\varphi$ satisfies $P O(W P O, S P O)$ with respect to $\|\cdot\|$.

(ii) $\varphi(p) \in \operatorname{conv}\{p(i): i \in N\}$ for all $p \in A^{N}$.

A nice consequence of theorem 2.1 is, that for the cases that $\|\cdot\|$ is strictly convex and $m=2$, Pareto optimality with respect to $\|\cdot\|$ is equivalent to a property which does not depend on the chosen norm. The next examples show that it is impossible to generalize this lemma. In example 2.8 we consider the same norm as in example 2.7. We show that Pareto optimality 
with respect to a norm which is not strictly convex, where $A=\mathbf{R}^{2}$, does not imply that $\varphi(p)$ is in the convex hull of the $p(i)$.

Example 2.8 Let $N=\{1,2\}, m=2$. Let $\|\cdot\|_{1}$ be the sum norm on $\mathbb{R}^{2}$, i.e., $\|x\|_{1} \equiv\left|x_{1}\right|+\left|x_{2}\right|$. Let $A=\mathbf{R}^{2}$. Let $\varphi: A^{2} \rightarrow A$ be the standard median scheme with one constant point $c^{1}=\left(\begin{array}{c}-\infty \\ -\infty\end{array}\right)$. Then $\varphi(x, y) \equiv$ $\left(\begin{array}{c}\min \left(x_{1}, y_{1}\right) \\ \min \left(x_{2}, y_{2}\right)\end{array}\right)$. Then $\|\varphi(x, y)-x\|_{1}+\|\varphi(x, y)-y\|_{1}=\left|x_{1}-\min \left(x_{1}, y_{1}\right)\right|+$ $\left|x_{2}-\min \left(x_{2}, y_{2}\right)\right|+\left|y_{1}-\min \left(x_{1}, y_{1}\right)\right|+\left|y_{2}-\min \left(x_{2}, y_{2}\right)\right|=\left|x_{1}-y_{1}\right|+\left|x_{2}-y_{2}\right|=$ $\|x-y\|_{1}$ for all $x, y \in \mathbf{R}^{2}$. Hence, if $\varphi$ is not Pareto optimal with respect to $\|\cdot\|_{1}$, there are $x, y, z \in \mathbb{R}^{2}$ such that $\|z-x\|_{1}+\|z-y\|_{1}<\|x-y\|_{1}$, which is impossible. Consequently, $\varphi$ satisfies $\mathrm{PO}$ with respect to $\|\cdot\|_{1}$. On the other hand,

$$
\varphi\left(\left(\begin{array}{l}
1 \\
0
\end{array}\right),\left(\begin{array}{l}
0 \\
1
\end{array}\right)\right)=\left(\begin{array}{l}
0 \\
0
\end{array}\right) \notin \operatorname{conv}\{x, y\} .
$$

So lemma 2.10 is not generally valid for norms which are not strictly convex.

Conjecture 2.2 Let $n \geq 2$. Let $A$ be a nonempty open subset of $\mathbf{R}^{2}$. Let $\|\cdot\|$ be a norm on $\mathbf{R}^{2}$ which is not strictly convex. Then there is a voting scheme $\varphi: A^{N} \rightarrow A$ which satisfies $P O$ with respect to $\|\cdot\|$ such that $\varphi(p) \notin \operatorname{conv}\{p(i): i \in N\}$ for some $p \in A^{N}$.

This conjecture is closely related to conjecture 2.1. Again, we leave it to the reader to confirm or refute this conjecture. The following example demonstrates that $\varphi(p) \in \operatorname{conv}\{p(i): i \in N\}$ for all $p \in\left(\mathbb{R}^{2}\right)^{N}$ does not necessarily imply PO with respect to $\|\cdot\|$, if $\|\cdot\|$ is not strictly convex.

Example 2.9 Let $N=\{1,2,3\}, m=2$. Let $\|\cdot\|_{1}$ be the sumnorm on $\mathbb{R}^{2}$. Let $\varphi:\left(\mathbb{R}^{2}\right)^{N} \rightarrow \mathbb{R}^{2}$ be the mean scheme i.e. $\varphi(p) \equiv \frac{1}{3} p(1)+\frac{1}{3} p(2)+\frac{1}{3} p(3)$. Then $\varphi(p) \in \operatorname{conv}\{p(1), p(2), p(3)\}$ for all $p \in\left(\mathbf{R}^{2}\right)^{N}$. Let $q \in\left(\mathbb{R}^{2}\right)^{N}$ be defined by $q(1)=\left(\begin{array}{l}0 \\ 0\end{array}\right), q(2)=\left(\begin{array}{l}1 \\ 0\end{array}\right)$ and $q(3)=\left(\begin{array}{l}0 \\ 1\end{array}\right)$. Then $\varphi(q)=$ $\left(\begin{array}{c}\frac{1}{3} \\ \frac{1}{3}\end{array}\right)$. So $\|0-q(1)\|_{1}<\|\varphi(q)-q(1)\|_{1},\|0-q(2)\|_{1}=1=\|\varphi(q)-q(2)\|_{1}$ and $\|0-q(3)\|_{1}=1=\|\varphi(q)-q(3)\|_{1}$. Consequently, $\varphi$ is not Pareto optimal with respect to $\|\cdot\|_{1}$. 
Conjecture 2.3 Let $n \geq 3$. Let $A$ be a nonempty open subset of $\mathbb{R}^{2}$. Let $\|$. $\|$ be a norm on $\mathbf{R}^{2}$ which is not strictly convex. Then there is a voting scheme $\varphi: A^{N} \rightarrow A$ which does not satisfy $P O$ with respect to $\|\cdot\|$ such that $\varphi(p) \in \operatorname{conv}\{p(i): i \in N\}$ for all $p \in A^{N}$.

To prove or disprove this conjecture is left to the reader.

For $m>2$, the restriction that $\|\cdot\|$ is strictly convex does not suffice for the truth of lemma 2.10.

We will construct a voting scheme $\tilde{\varphi}: A^{N} \rightarrow A$ which satisfies PO with respect to $\|\cdot\|_{p}$, such that $\tilde{\varphi}(q) \notin \operatorname{conv}\{q(i): i \in N\}$ for some $q \in A^{N}$, under the restriction that $p \neq 2$.

Example 2.10 Let $N=\{1,2,3\}, m=3$. Let $\|\cdot\|_{p}$ be the $p$-norm on $\mathbf{R}^{3}$, i.e. $\|x\|=\left(\sum_{i=1}^{3}\left|x_{i}\right|^{p}\right)^{1 / p}$, with $p \in(1, \infty)$. It is easy to verify that $\|\cdot\|_{p}$ is a strictly convex norm. Let $A=\mathbb{R}^{3}$.

Let $\varphi: A^{N} \rightarrow A$ be an arbitrary scheme which satisfies $\mathrm{PO}$ with respect to $\|\cdot\|_{p}$. Let

$$
q=\left(\left(\begin{array}{l}
1 \\
0 \\
0
\end{array}\right),\left(\begin{array}{l}
0 \\
1 \\
0
\end{array}\right),\left(\begin{array}{l}
0 \\
0 \\
1
\end{array}\right)\right), \lambda=1-\frac{1}{1+2^{1 /(p-1)}}
$$

Let $\tilde{\varphi}:\left(\mathbb{R}^{3}\right)^{N} \rightarrow \mathbb{R}^{3}$ be such that $\tilde{\varphi}(r)=\varphi(r)$ for all $r \in\left(\mathbb{R}^{3}\right)^{N} \backslash\{q\}$ and $\tilde{\varphi}(q)=\lambda\left(\begin{array}{l}1 \\ 1 \\ 1\end{array}\right)$.

Now suppose $\tilde{\varphi}$ is not Pareto optimal with respect to $\|\cdot\|_{p}$. In view of proposition 2.1 there is an $x \in \mathbf{R}^{3}$ with $\|x-q(i)\|<\left\|\lambda\left(\begin{array}{l}1 \\ 1 \\ 1\end{array}\right)-q(i)\right\|_{p}$ for $i=1,2,3$. Let

$$
\tilde{x}=\left(\begin{array}{c}
x_{3} \\
x_{1} \\
x_{2}
\end{array}\right), \bar{x}=\left(\begin{array}{l}
x_{2} \\
x_{3} \\
x_{1}
\end{array}\right) .
$$

By symmetry, we have for $i=1,2,3$ that

$$
\|\tilde{x}-q(i)\|_{p}<\left\|\left(\begin{array}{c}
\lambda \\
\lambda \\
\lambda
\end{array}\right)-q(i)\right\|_{p}
$$


and

$$
\|\bar{x}-q(i)\|_{p}<\left\|\lambda\left(\begin{array}{l}
1 \\
1 \\
1
\end{array}\right)-q(i)\right\|_{p}
$$

Let $y=\frac{1}{3} x+\frac{1}{3} \tilde{x}+\frac{1}{3} \bar{x}$. Hence, $\|y-q(i)\|_{p}<\left\|\lambda\left(\begin{array}{l}1 \\ 1 \\ 1\end{array}\right)-q(i)\right\|_{p}$ for $i=1,2,3$. So

$$
\left\|y_{1}\left(\begin{array}{l}
1 \\
1 \\
1
\end{array}\right)-q(1)\right\|_{p}<\left\|\lambda\left(\begin{array}{l}
1 \\
1 \\
1
\end{array}\right)-q(1)\right\|_{p}
$$

Let $f: \mathbb{R} \rightarrow \mathbb{R}$ with $f(\mu)=\left\|\mu\left(\begin{array}{l}1 \\ 1 \\ 1\end{array}\right)-q(1)\right\|_{p}, \mu \in \mathbb{R}$. So, $f(\mu)=$ $|\mu-1|^{p}+2|\mu|^{p}$. It is elementary to verify that $f$ has a unique global minimum over $\mathbb{R}$ at $\mu=\lambda$. This contradicts (2.5). Consequently, $\tilde{\varphi}$ satisfies PO (with respect to $\left.\|\cdot\|_{p}\right)$.

On the other hand, $\varphi(q) \in \operatorname{conv}\{q(1), q(2), q(3)\}$ if and only if $\lambda=\frac{1}{3}$. This is the case if and only if $p=2$, which corresponds to the Euclidean norm. So, if $p \neq 2, \varphi$ is Pareto optimal, but $\varphi(q)$ is not in the convex hull of the $q(i)(i \in N)$.

On the other hand let $\hat{\varphi}:\left(\mathbb{R}^{3}\right)^{N} \rightarrow \mathbb{R}^{3}$ be the mean scheme, i.e. $\hat{\varphi}(r)=$ $\frac{1}{3} r(1)+\frac{1}{3} r(2)+\frac{1}{3} r(3)$ for all $r \in\left(\mathbb{R}^{3}\right)^{N}$. Then $\hat{\varphi}(r) \in \operatorname{conv}\{r(1), r(2), r(3)\}$ for all $r \in\left(\mathbb{R}^{3}\right)^{N}$. Furthermore $\hat{\varphi}(q)=\frac{1}{3}\left(\begin{array}{l}1 \\ 1 \\ 1\end{array}\right)$. So if $p \neq 2$, then $\hat{\varphi}$ is not Pareto optimal with respect to $\|\cdot\| p$, since $\left\|\lambda\left(\begin{array}{l}1 \\ 1 \\ 1\end{array}\right)-q(i)\right\|_{p}<$ $\left\|\frac{1}{3}\left(\begin{array}{l}1 \\ 1 \\ 1\end{array}\right)-q(i)\right\|_{p}$ for $i=1,2,3$

Although theorem 2.1 cannot be generalized to higher dimensions or for all norms on $\mathbb{R}^{2}$, we will discuss another case where Pareto optimality is equivalent to the property that the scheme point is in the convex hull of the ideal points for all profiles $p$. We postpone the discussion on these matters to the relevant section (see lemma 3.3). However, by restricting ourselves to 
a special class of profiles, namely the class of all profiles such that the $p(i)$ are collinear, we obtain:

Lemma 2.11 Let $A$ be an open subset of $\mathbf{R}^{m}$. Let $\|\cdot\|$ be a norm on $\mathbf{R}^{m}$. Let $\varphi: A^{N} \rightarrow A$ satisfy $S P O$ with respect to $\|\cdot\|$. Let $p \in A^{N}$ be such that all $p(i)$ are collinear. Then $\varphi(p) \in \operatorname{conv}\{p(1), \ldots, p(n)\}$.

Proof Suppose $\varphi(p) \notin\{p(1), \ldots, p(n)\}$. Let $k, l \in\{1, \ldots, n\}$ be such that $p(i) \in[p(k), p(l)]$ for all $i \in N$. Then by the triangular inequality for norms, $\|p(k)-\varphi(p)\|+\|p(l)-\varphi(p)\| \geq\|p(l)-p(k)\|$. Let

$$
\lambda=\frac{\|p(k)-\varphi(p)\|}{\|p(k)-\varphi(p)\|+\|p(l)-\varphi(p)\|}, y=\lambda p(k)+(1-\lambda) p(l) .
$$

Then $\|p(k)-y\| \leq\|p(k)-\varphi(p)\|,\|p(l)-y\| \leq\|p(l)-\varphi(p)\|$ and $y \in$ $(p(k), p(l))$. If $p(i) \in[p(k), y]$, then $\|p(i)-y\|=\|p(k)-y\|-\|p(i)-p(k)\| \leq$ $\|p(k)-\varphi(p)\|-\|p(i)-p(k)\| \leq\|p(i)-\varphi(p)\|$. Similarly, $\|p(i)-y\| \leq$ $\|p(i)-\varphi(p)\|$ if $p(i) \in[p(l), y]$. Hence, $\|p(i)-y\| \leq\|p(i)-\varphi(p)\|$ for all $i \in N$. By the openness of $A$ we can take $z \in(y, \varphi(p))$ with $z \in A$. Then by lemma $\mathrm{A1}$ (ii), $\|p(i)-z\| \leq\|p(i)-\varphi(p)\|$ for all $i \in N$. This contradicts SPO.

The following lemma follows straightforwardly from lemma 2.11 and proposition 2.1 .

Lemma 2.12 Let $A$ be an open subset on $\mathbb{R}^{m}$. Let $\|\cdot\|$ be a strictly convex norm on $\mathbb{R}^{m}$. Let $\varphi: A^{N} \rightarrow A$ satisfy $P O(W P O)$ with respect to $\|\cdot\|$. Let $p \in A^{N}$ be such that all $p(i)$ are collinear. Then $\varphi(p) \in \operatorname{conv}\{p(1), \ldots, p(n)\}$.

We now discuss some implication of strategy-proofness with respect to (strictly convex) norms.

Lemma 2.13 states that strategy-proofness with respect to a strictly norm implies positive association. A voting scheme $\varphi: A^{N} \rightarrow A$ satisfies positive association, if for all $p, \hat{p} \in A^{N}$ with $\hat{p}(i) \in[\varphi(p), p(i)]$ for all $i \in N$ we have $\varphi(\hat{p})=\varphi(p)$. So positive association means that moving (some of) the individually reported points into the direction of the compromise point, does not change the compromise point.

Note that positive association implies confirmatoriness.

Lemma 2.13 Let $A \subset \mathbf{R}^{m}$. Let $\|\cdot\|$ be a strictly convex norm on $\mathbb{R}^{m}$. Let $\varphi \in A^{N} \rightarrow A$ be strategy-proof with respect to $\|\cdot\|$. Then $\varphi$ satisfies positive association. 
Proof Let $p, \hat{p} \in A^{N}$ and $i \in N$ with $\hat{p}(i) \in[\varphi(p), p(i)]$ and $p=N \backslash\{i\} \hat{p}$. It is sufficient to prove that $\varphi(\hat{p})=\varphi(p)$. For such profiles, by SP:

$$
\|p(i)-\varphi(p)\| \leq\|p(i)-\varphi(\hat{p})\|
$$

and

$$
\|\hat{p}(i)-\varphi(\hat{p})\| \leq\|\hat{p}(i)-\varphi(p)\| .
$$

If $\hat{p}(i) \notin \operatorname{conv}\{p(i), \varphi(\hat{p})\}$ then by strict convexity of $\|\cdot\|$,

$$
\|p(i)-\varphi(\hat{p})\|<\|p(i)-\hat{p}(i)\|+\|\hat{p}(i)-\varphi(\hat{p})\| .
$$

Hence by $(2.7)$,

$$
\begin{aligned}
\|p(i)-\varphi(\hat{p})\| & <\|p(i)-\hat{p}(i)\|+\|\hat{p}(i)-\varphi(p)\| \\
& =\|p(i)-\varphi(p)\| .
\end{aligned}
$$

This contradicts (2.6), hence $\hat{p}(i) \in[p(i), \varphi(\hat{p})]$. Consequently, (2.6) and (2.7) imply $\varphi(\hat{p})=\varphi(p)$.

In the above lemma the restriction that $\|\cdot\|$ is strictly convex, is essential. If $A$ is a nonempty open subset of $\mathbb{R}^{m}$, and $\|\cdot\|$ is a norm on $\mathbb{R}^{m}$ which is not strictly convex, we can construct a voting scheme which satisfies SP with respect to $\|\cdot\|$, but not positive association:

Lemma 2.14 Let $A$ be a nonempty open subset of $\mathbf{R}^{m}$. Let $\|\cdot\|$ be a norm on $\mathrm{R}^{m}$ which is not strictly convex. Then there is a voting scheme $\varphi: A^{N} \rightarrow A$ which satisfies $S P$ with respect to $\|\cdot\|$, but not positive association.

Proof Since $\|\cdot\|$ is a norm on $\mathbb{R}^{m}$ which is not strictly convex, there are different $u, v \in \mathbb{R}^{m}$ with $\|u\|=1,\|v\|=1$ and $\|u+v\|=2$ (see figure 2.5 for an example of such a norm). Let $a \in A$. Since $A$ is open we can take $\varepsilon>0$ such that $\left\{x \in \mathbb{R}^{m} ;\|x-a\| \leq \varepsilon\right\} \subset A$. Let $\hat{u}=a+\varepsilon u, \hat{v}=a+\varepsilon v$. Let $\varphi: A^{N} \rightarrow A$ be defined by

$$
\begin{aligned}
\varphi(p)= & \hat{u} \quad \text { if } p(1)=a, \\
\varphi(p) \in & (\leftarrow, p(1), p(1)+v, \rightarrow) \cap[\hat{u}, \hat{v}] \text { if } \\
& \quad(\leftarrow, p(1), p(1)+v, \rightarrow) \cap[\hat{u}, \hat{v}] \neq \emptyset \text { and } p(i) \neq a, \\
\varphi(p)= & \hat{u} \text { if }(\leftarrow, p(1), p(1)+v, \rightarrow) \cap[\hat{v}, \hat{u}, \rightarrow) \backslash[\hat{u}, \hat{v}] \neq \emptyset, \\
\varphi(p)= & \hat{v} \text { if }(\leftarrow, p(1), p(1)+v, \rightarrow) \cap[\hat{u}, \hat{v}, \rightarrow) \backslash[\hat{u}, \hat{v}] \neq \emptyset .
\end{aligned}
$$




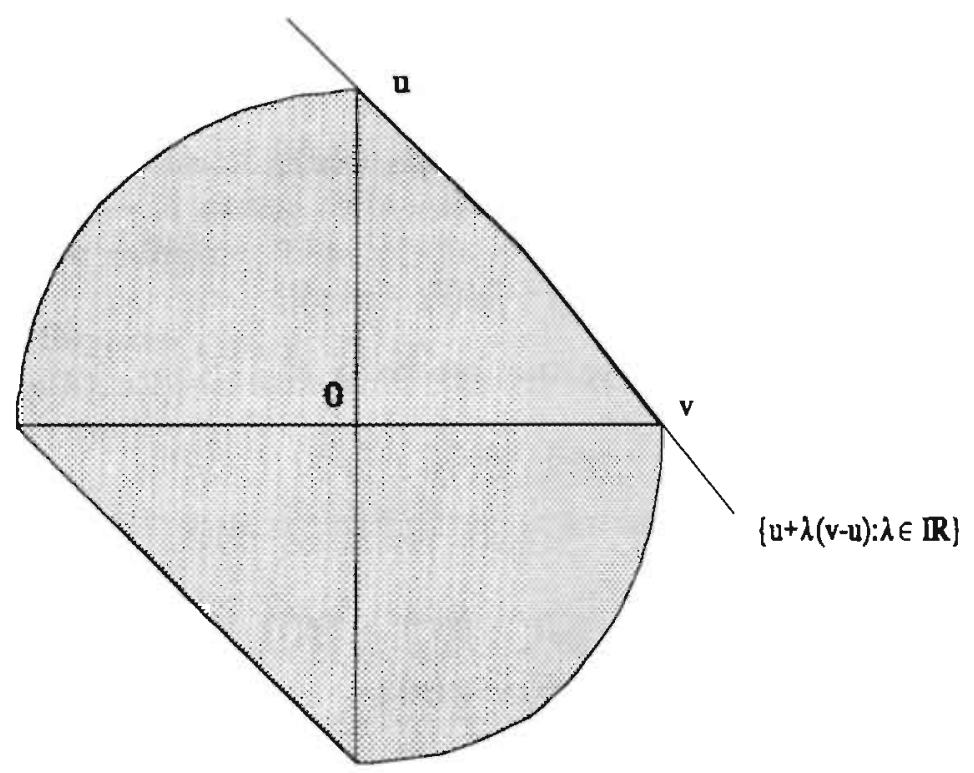

Figure 2.5: A norm which is not strictly convex.

So $\varphi(p)$ depends only on the reported point of agent 1 . Furthermore, $\varphi(p) \epsilon$ $[\hat{u}, \hat{v}]$ for all $p \in A^{N}$.

Let $q, \hat{q} \in A^{N}$ with $q(1)=a, \hat{q}(1)=\frac{1}{2} a+\frac{1}{2} \hat{u}$ and $q={ }_{N \backslash\{1\}} \hat{q}$. Then $\varphi(q)=\hat{u}$ and $\varphi(\hat{q})=\frac{1}{2} \hat{u}+\frac{1}{2} \hat{v}$. Since $\hat{q}(i) \in[\hat{u}, q(i)]$ for all $i \in N$, this contradicts positive association. So $\varphi$ does not satisfy positive association.

Lemma A1 (v) (see Appendix A) implies that $u$ and $v$ are $\|\cdot\|$-orthogonal to $u-v$ in the sense of Birkhoff. (This fact is also demonstrated by figure 2.5: the sets $\left\{x \in \mathbf{R}^{m}:|| x||<1\right\}$ (the shaded area in the picture) and $\{u+\lambda(u-v): \lambda \in \mathbb{R}\}$ are disjoint.) Consequently, $u$ and $v$ are $\|\cdot\|$-orthogonal to $\hat{u}-\hat{v}$ in the sense of Birkhoff. These facts imply that $\|p(1)-\varphi(p)\| \leq\|p(1)-x\|$ for all $p \in A^{N}$, and all $x \in[\hat{u}, \hat{v}]$. Since agent 1 is the only one who can influence the compromise point, this implies that $\varphi$ is strategy-proof with respect to $\|\cdot\|$.

Strong strategy-proofness with respect to a strictly convex norm however is a sufficient condition for positive association:

Lemma 2.15 Let $A \subset \mathbb{R}^{m}$. Let $\|\cdot\|$ be a norm on $\mathbf{R}^{m}$. Let $\varphi \in A^{N} \rightarrow A$ satisfy $S S P$ with respect to $\|\cdot\|$. Then $\varphi$ satisfies positive association. 
Proof Let $p, \hat{p} \in A^{N}$ and $i \in \mathbb{N}$ with $\hat{p}(i) \in[\varphi(p), p(i)]$ and $p=N \backslash\{i\} \hat{p}$. It is sufficient to prove that $\varphi(\hat{p})=\varphi(p)$. By SP, $\|\hat{p}(i)-\varphi(\hat{p})\| \leq\|\hat{p}(i)-\varphi(p)\|$. Hence,

$$
\begin{aligned}
\|p(i)-\varphi(p)\| & =\|p(i)-\hat{p}(i)\|+\|\hat{p}(i)-\varphi(p)\| \\
& \geq\|p(i)-\hat{p}(i)\|+\|\hat{p}(i)-\varphi(\hat{p})\| \\
& \geq\|p(i)-\varphi(\hat{p})\| .
\end{aligned}
$$

So by SSP, $\varphi(\hat{p})=\varphi(p)$.

Strategy-proofness with respect to a norm does not imply componentwise continuity of a voting scheme. It is strong enough however to imply a weaker continuity property:

Lemma 2.16 Let $A \subset \mathbb{R}^{m}$. Let $\|\cdot\|$ be a norm on $\mathbb{R}^{m}$. Let $\varphi$ satisfy $S P$ with respect to $\|\cdot\|$. Let $i \in N$. Let $p^{l} \in A(l \in \mathbb{N})$ with $p^{l}=N \backslash\{i\} p^{0}$ and $\left\|p^{l}(i)-p^{0}(i)\right\| \rightarrow 0$. Then $\left\|p^{0}(i)-\varphi\left(p^{l}\right)\right\| \rightarrow\left\|p^{0}(i)-\varphi\left(p^{0}\right)\right\|$.

Proof By SP,

$$
\begin{aligned}
\left\|p^{0}(i)-\varphi\left(p^{l}\right)\right\| & \leq\left\|p^{0}(i)-p^{l}(i)\right\|+\left\|p^{l}(i)-\varphi\left(p^{l}\right)\right\| \\
& \leq\left\|p^{0}(i)-p^{l}(i)\right\|+\left\|p^{l}(i)-\varphi\left(p^{0}\right)\right\| \\
& \leq 2\left\|p^{0}(i)-p^{l}(i)\right\|+\left\|p^{0}(i)-\varphi\left(p^{0}\right)\right\|
\end{aligned}
$$

for all $l \in \mathbf{N}$. Also by SP, $\left\|p^{0}(i)-\varphi\left(p^{0}\right)\right\| \leq\left\|p^{0}(i)-\varphi\left(p^{l}\right)\right\|$ for all $l \in \mathbf{N}$. Consequently, since $\left\|p^{0}(i)-p^{l}(i)\right\| \rightarrow 0$, we have that $\left\|p^{0}(i)-\varphi\left(p^{l}\right)\right\| \rightarrow$ $\left\|p^{0}(i)-\varphi\left(p^{0}\right)\right\|$.

Lemma 2.18 is very similar to lemma 5.10 , which is on strict strategyproofness. This lemma states that SSP with respect to a norm is a sufficient condition for componentwise continuity, provided that $A$ is a closed subset of $\mathbf{R}^{m}$.

In the foregoing we discussed some implications of strategy-proofness and Pareto optimality with respect to a norm. In the remainder of this section we investigate under what conditions coordinatewise median schemes (see section 1.2) satisfy SP and PO.

Lemma 2.17 Let $A$ be a nonempty subset of $\mathbb{R}^{m}$. Let $\|\cdot\|$ be a norm on $\mathbf{R}^{m}$. Let $k \in \mathbb{N}$. Let $u^{1}, \ldots, u^{m} \in \mathbb{R}^{m}$ be linearly independent. Let $\varphi: A^{N} \rightarrow A$ be 
a coordinatewise median scheme with respect to $\left\{u^{i}: i \in M\right\}$ with $k$ constant points $c^{i} \in \overline{\mathbf{R}}^{m}$. Let $\left\{u^{i}: i \in M\right\}$ be $\|\cdot\|$-orthogonal. Then $\varphi$ satisfies $S P$ with respect to $\|\cdot\|$.

Proof Since $\left\{u^{1}, \ldots, u^{m}\right\}$ is a basis of $\mathbb{R}^{m}$, we can define $\lambda_{j}: \mathbb{R}^{m} \rightarrow \mathbb{R}$ $(j=1, \ldots, m)$ uniquely such that $x=\sum_{j=1}^{m} \lambda_{j}(x) u^{j}$ for all $x \in \mathbb{R}^{m}$. Hence, we can write: $\varphi(p)=\sum_{j=1}^{m} \operatorname{med}\left(\lambda_{j}(p(1)), \ldots, \lambda_{j}(p(n)), c_{j}^{1}, \ldots, c_{j}^{k}\right) u^{j}$.

Let $i \in N$, and let $p, \hat{p}$ be profiles with $p(j)=\hat{p}(j)$ for all $j \neq i$. Let $\alpha_{j}=\lambda_{j}(\varphi(p)), \hat{\alpha}_{j}=\lambda_{j}(\varphi(\hat{p})), j=1, \ldots, m$. Then by definition of the median, $\hat{\alpha}_{j} \leq \alpha_{j} \leq \lambda_{j}(p(i))$ or $\lambda_{j}(p(i)) \leq \alpha_{j} \leq \hat{\alpha}_{j}$ for all $j=1, \ldots, m$. Since $\left\{u^{1}, \ldots, u^{m}\right\}$ is $\|\cdot\|$-orthogonal, we obtain by lemma 2.2 ,

$$
\begin{aligned}
\|p(i)-\varphi(p)\| & =\left\|p(i)-\sum_{j=1}^{m} \alpha_{j} u^{j}\right\| \\
& =\left\|\sum_{j=1}^{m}\left(\lambda_{j}(p(i))-\alpha_{j}\right) u^{j}\right\| \\
& \leq\left\|\sum_{j=1}^{m}\left(\lambda_{j}(p(i))-\hat{\alpha}_{j}\right) u^{j}\right\| \\
& =\|p(i)-\varphi(\hat{p})\| .
\end{aligned}
$$

Consequently, $\varphi$ is strategy-proof with respect to $\|\cdot\|$.

Lemma 2.18 Let $\|\cdot\|$ be a norm on $\mathbb{R}^{m}$. Let $u^{1}, \ldots, u^{m} \in \mathbb{R}^{m}$ be linearly independent. Let $\varphi:\left(\mathbf{R}^{m}\right)^{N} \rightarrow \mathbb{R}^{m}$ be a coordinatewise median scheme with respect to $\left\{u^{1}, \ldots, u^{m}\right\}$ with $n+1$ constant points $c^{i} \in \overline{\mathbb{R}}^{m}$, where $\left\{c_{j}^{1}, \ldots, c_{j}^{n+1}\right\}$ contains at least three elements for all $j=1, \ldots, m$. Then $\varphi$ satisfies strategy-proofness with respect to $\|\cdot\|$ if and only if $\left\{u^{1}, \ldots, u^{m}\right\}$ is $\|\cdot\|$-orthogonal.

Proof From lemma 2.17 we know that $\varphi$ satisfies strategy-proofness if $\left\{u^{1}, \ldots, u^{m}\right\}$ is $\|\cdot\|$-orthogonal. Conversely, suppose $\left\{u^{1}, \ldots, u^{m}\right\}$ is not $\|\cdot\|$-orthogonal. So there are $k \in\{1, \ldots, m\}$, and $\tau_{i} \in \mathbb{R}(i=1, \ldots, m)$ such that

$$
\left\|\sum_{j=1}^{m} \tau_{j} u^{j}\right\|<\left\|\sum_{\substack{j=1 \\ i \neq k}}^{m} \tau_{j} u^{j}\right\| .
$$

W.l.o.g. assume that $\tau_{j} \geq 0$ for $j=1, \ldots, m$. Let

$$
\mu_{j} \in\left(\min _{i \in\{1, \ldots, m\}} c_{j}^{i} \max _{i \in\{1, \ldots, m\}} c_{j}^{i}\right)
$$


be such that $\left|\left\{i=1, \ldots, n+1: c_{j}^{i} \leq \mu_{j}\right\}\right| \geq 2$. W.l.o.g. we assume that the $\tau_{j}$ are so small that $c_{j}^{i} \notin\left(\mu_{j}, \mu_{j}+\tau_{j}\right]$ for all $i=1, \ldots, n+1, j=1, \ldots, m$. Let $l=\left|\left\{i \in\{1, \ldots, n+1\}: c_{k}^{i}>\mu_{k}\right\}\right|$. Let the profile $p$ be such that

$$
\begin{aligned}
& p(i)=\sum_{j=1}^{m} \mu_{j} u^{j}, i=1, \ldots, l-1, \\
& p(i)=\tau_{k} u^{k}+\sum_{j=1}^{m} \mu_{j} u^{j}, i=l, \ldots, n-1
\end{aligned}
$$

and

$$
p(n)=\sum_{j=1}^{m}\left(\mu_{j}+\tau_{j}\right) u^{j} .
$$

Let the profile $\tilde{p}$ be such that $\tilde{p}(i)=p(i), i=1, \ldots, n-1$ and $\tilde{p}(n)=$ $\sum_{j=1}^{m} \mu_{j} u^{j}$. Then

$$
\varphi(p)=\sum_{j=1}^{m} \mu_{j} u^{j}+\tau_{k} u^{k} \text { and } \varphi(\tilde{p})=\sum_{j=1}^{m} \mu_{j} u^{j}
$$

So,

$$
\|p(n)-\varphi(\tilde{p})\|=\left\|\sum_{j=1}^{m} \tau_{j} u^{j}\right\|<\left\|\sum_{\substack{j=1 \\ j \neq k}}^{m} \tau_{j} \mathbf{u}^{j}\right\|=\|p(n)-\varphi(p)\| .
$$

This contradicts strategy-proofness. Consequently, if $\varphi$ is strategy-proof, $\left\{u^{1}, \ldots, u^{m}\right\}$ is orthogonal.

The following lemma can be proved in the same way.

Lemma 2.19 Let $n>1$. Let $\|\cdot\|$ be a norm on $\mathbb{R}^{m}$. Let $u^{1}, \ldots, u^{m} \in$ $\mathbf{R}^{m}$ be linearly independent. Let $\varphi:\left(\mathbf{R}^{m}\right)^{N} \rightarrow \mathbf{R}^{m}$ be a coordinatewise median scheme with respect to $\left\{u^{1}, \ldots, u^{m}\right\}$ without constant points. Then $\varphi$ satisfies strategy-proofness with respect to $\|\cdot\|$ if and only if $\left\{u^{1}, \ldots, u^{m}\right\}$ is $\|\cdot\|$-orthogonal.

Lemma 2.20 Let $\|\cdot\|$ be a strictly convex norm on $\mathbf{R}^{2}$. Let $u^{1}, u^{2} \in \mathbf{R}^{2}$ be linearly independent. Let $\varphi:\left(\mathbf{R}^{2}\right)^{N} \rightarrow \mathbb{R}^{2}$ be a coordinatewise median scheme with respect to $\left\{u^{1}, u^{2}\right\}$ without constant points. Then $\varphi$ is Pareto optimal with respect to $\|\cdot\|$. 
Proof Let $\lambda(x)$ and $\mu(x)$ be the coordinates of $x$ with respect to the basis $\left\{u^{1}, u^{2}\right\}$. In other words, $\lambda, \mu: \mathbb{R}^{2} \rightarrow \mathbb{R}$ are such that $x=\lambda(x) \cdot u^{1}+\mu(x) \cdot u^{2}$ for all $x \in \mathbf{R}^{2}$. Let $i, j \in N$ with $\lambda(p(i))=\lambda(\varphi(p))$ and $\mu(p(j))=\mu(\varphi(p))$. W.l.o.g. suppose $\mu(p(i)) \leq \mu(\varphi(p))$, and $\lambda(p(j)) \leq \lambda(\varphi(p))$. In order to prove Pareto optimality of $\varphi$, it is, in view of theorem 2.1 , sufficient to show that there must be a $k \in N$ with $\lambda(p(k)) \geq \lambda(\varphi(p))$ and $\mu(p(k)) \geq \mu(\varphi(p))$.

We will argue from contradiction: suppose such an $l$ does not exist. Since there must be at least $\frac{n+1}{2}$ agents $k \in N$ with $\mu(p(k)) \geq \mu(\varphi(p))$, we have:

$$
|\{l \in N: \mu(p(l)) \geq \mu(\varphi(p)), \lambda(p(l))<\lambda(\varphi(p))\}| \geq \frac{n+1}{2} .
$$

Similarly:

$$
|\{l \in N: \lambda(p(l)) \geq \lambda(\varphi(p)), \mu(p(l))<\mu(\varphi(p))\}| \geq \frac{n+1}{2} .
$$

Since these sets are disjoint, we conclude $n \geq n+1$, which is impossible. This establishes the desired contradiction. Thus, $\varphi$ is Pareto optimal.

In lemma 2.20 the strict convexity of $\|\cdot\|$ is essential, as is shown by the following example.

Example 2.11 Let $n=3$. Let $\varphi:\left(\mathbb{R}^{2}\right)^{N} \rightarrow \mathbb{R}^{2}$ be the coordinatewise median scheme with respect to the standard unity vectors without constant points.

Let $\|\cdot\|_{\infty}$ be the maximumnorm on $\mathbb{R}^{2}$, i.e. $\|x\|_{\infty}=\max _{j=1,2}\left|x_{j}\right|$. Let $p=\left(\left(\begin{array}{l}0 \\ 1\end{array}\right),\left(\begin{array}{c}0 \\ -1\end{array}\right),\left(\begin{array}{l}1 \\ 0\end{array}\right)\right)$. Then $\varphi(p)=0$. On the other hand,

$$
\begin{aligned}
& \left\|\left(\begin{array}{l}
1 \\
0
\end{array}\right)-p(1)\right\|_{\infty}=\|\varphi(p)-p(1)\|_{\infty}=1 \\
& \left\|\left(\begin{array}{l}
1 \\
0
\end{array}\right)-p(2)\right\|_{\infty}=\|\varphi(p)-p(2)\|_{\infty}=1
\end{aligned}
$$

and

$$
\left\|\left(\begin{array}{l}
1 \\
0
\end{array}\right)-p(3)\right\|_{\infty}=0<\|\varphi(p)-p(3)\|_{\infty}=1 .
$$

Consequently, $\varphi$ is not Pareto optimal with respect to $\|\cdot\|$.

So lemma 2.20 does not hold for arbitrary norms on $\mathbb{R}^{2}$. It is also impossible to generalize this lemma for $m \geq 3$, as is demonstrated by the following example. 
Example 2.12 Let $m=3, n=3$. Let $\varphi:\left(\mathbf{R}^{3}\right)^{N} \rightarrow \mathbb{R}^{3}$ be the coordinatewise median scheme with respect to the standard unity vectors without constant points. Let $\|\cdot\|_{2}$ denote the Euclidean norm, which is strictly convex. Let $p=\left(\left(\begin{array}{l}1 \\ 0 \\ 0\end{array}\right),\left(\begin{array}{l}0 \\ 1 \\ 0\end{array}\right),\left(\begin{array}{l}0 \\ 0 \\ 1\end{array}\right)\right)$. Then $\varphi(p)=0$. Hence, $\left\|\left(\begin{array}{c}\frac{1}{3} \\ \frac{1}{3} \\ \frac{1}{3}\end{array}\right)-p(i)\right\|_{2}=\sqrt[2]{\left(\frac{2}{3}\right)^{2}+\left(\frac{1}{3}\right)^{2}+\left(\frac{1}{3}\right)^{2}}=\frac{1}{3} \sqrt{5}<1=\|0-p(i)\|_{2}$ for all $i \in N$. Consequently, $\varphi$ is not Pareto optimal with respect to $\|\cdot\|_{2}$.

For $m \geq 2$, a coordinatewise median scheme on $\mathbf{R}^{m}$ is not strongly Pareto optimal with respect to a norm on $\mathbb{R}^{m}$, except if it coincides with a coordinatewise median scheme without constant points:

Lemma 2.21 Let $m \geq 2$. Let $\|\cdot\|$ be a norm on $\mathbb{R}^{m}$. Let $\varphi:\left(\mathbb{R}^{m}\right)^{N} \rightarrow \mathbb{R}^{m}$ be a coordinatewise median scheme which satisfies $S P O$ with respect to $\|\cdot\|$. Then $\varphi$ is a coordinatewise median scheme without constant points.

Proof Let linearly independent $u^{1}, \ldots, u^{m} \in \mathbb{R}^{m}$ be such that $\varphi$ is the coordinatewise median scheme with respect to $\left\{u^{1}, \ldots, u^{m}\right\}$ and constant points $c^{1}, \ldots, c^{n+1}$. Suppose $\varphi$ cannot be written as a coordinatewise median scheme without constant points. Then we can take $j \in\{1, \ldots, m\}$ such that

$$
\left|\left\{i \in\{1, \ldots, n+1\}: c_{j}^{i}=\infty\right\}\right|<\frac{n+1}{2}
$$

or

$$
\left|\left\{i \in\{1, \ldots, n+1\}: c_{j}^{i}=-\infty\right\}\right|<\frac{n+1}{2} .
$$

W.l.o.g. assume that

$$
\left|\left\{i \in\{1, \ldots, n+1\}: c_{j}^{i}=\infty\right\}\right|<\frac{n+1}{2} .
$$

So we can choose $\alpha \in \mathbf{R}$ such that

$$
\left|\left\{i \in\{1, \ldots, n+1\}: c_{j}^{i} \geq \alpha\right\}\right|<\frac{n+1}{2} .
$$

Let $k \in\{1, \ldots, m\} \backslash\{j\}$. W.l.o.g. we assume that

$$
\left|\left\{i \in\{1, \ldots, n+1\}: c_{k}^{i}>0\right\}\right| \leq \frac{n+1}{2} .
$$

If $c_{j}^{i}<\alpha$ for all $i \in N$ then $\varphi\left(\left(\alpha u^{j}\right)^{N}\right) \neq \alpha u^{j}$, which contradicts PO. Hence,

$$
\left|\left\{i \in\{1, \ldots, n+1\}: c_{j}^{i} \geq \alpha\right\}\right| \geq 1 .
$$


So by $(2.8), n>1$. By a similar argument,

$$
\left|\left\{i \in\{1, \ldots, n+1\}: c_{k}^{i} \geq 0\right\}\right| \geq 1 \text {. }
$$

Let $l=\frac{n}{2}$ if $n$ is even and $l=\frac{n-1}{2}$ if $n$ is odd. Let $p \in\left(\mathbf{R}^{m}\right)^{N}$ be such that $p(i)=\alpha u^{j}+u^{k}$ for $i=1, \ldots, l$ and $p(i)=\alpha u^{j}+u^{j}$ for $i=l+1, \ldots, n$. Then $\varphi(p)=\alpha u^{j} \notin\left[\alpha u^{j}+u^{k}, \alpha u^{j}+u^{j}\right]$. This contradicts lemma 2.9. Consequently, $\varphi$ is a coordinatewise median scheme without constant points.

The following corollary is a direct consequence of lemma 2.21 and theorem 2.1.

Corollary 2.5 Let $m \geq 2$. Let $\|\cdot\|$ be a strictly convex norm on $\mathbb{R}^{m}$. Let $\varphi:\left(\mathbb{R}^{m}\right)^{N} \rightarrow \mathbb{R}^{m}$ be a coordinatewise median scheme which satisfies $P O$ or WPO with respect to $\|\cdot\|$. Then $\varphi$ is a coordinatewise median scheme without constant points.

\subsection{Two person location problems in normed spaces}

Let $A$ be a nonempty open subset of $\mathbb{R}^{m}$. In this section we will study $\varphi: A^{2} \rightarrow A$, with $m \geq 2$ and $\varphi$ assumed to satisfy SP and SPO with respect to a norm on $\mathbb{R}^{m}$. The first main result is theorem 2.2 , which states that $\varphi$ must be dictatorial.

Next it will be discussed how this theorem can be applied for cases with an arbitrary even number of agents. Theorem 2.3 states that in this case there are no voting schemes which satisfy SP and SPO with respect to a norm and $\mathrm{AN}$.

Under the assumption that the considered norm is strictly convex, we obtain the same results by relaxing SPO to PO or WPO (here we use proposition 2.1). Throughout this section we use the notation $\varphi(u, v)$ instead of $\varphi(p)$, where $u=p(1)$ and $v=p(2)$.

Lemma 2.22 Let $A$ be a nonempty open subset of $\mathbf{R}^{m}, m \geq 2$. Let $\varphi$ : $A^{2} \rightarrow A$ satisfy $S P O$ with respect to a norm $\|\cdot\|$. Then $\varphi(u, v) \in[u, v]$ for all $u, v \in A$.

Proof Since every pair $u, v \in A$ is collinear, lemma 2.9 implies that $\varphi(u, v) \in[u, v]$ for all $u, v \in A$.

The following assumption will be used in lemmas 2.23-2.32. 
Assumption 2.1 Let $A$ be a nonempty open subset of $\mathbb{R}^{m}, m \geq 2$. Let $\|\cdot\|$ be a norm on $\mathbf{R}^{m}$. Let $\varphi: A^{2} \rightarrow A$ satisfy $S P$ and $S P O$ with respect to $\|\cdot\|$.

Lemma 2.23 Let assumption 2.1. Then $\varphi$ satisfies positive association.

Proof Let $u, v \in A$. Let $w=\varphi(u, v)$. Lemma 2.23 implies that

$$
w \in[u, v]
$$

Let

$$
\hat{u} \in[u, w]
$$

and $\hat{v} \in[v, w]$. We will first prove that $\varphi(\hat{u}, v)=w$. Let $\hat{w}=\varphi(\hat{u}, v)$. Then by lemma 2.22 we have that

$$
\hat{w} \in[\hat{u}, v] .
$$

SP with respect to $\|\cdot\|$ implies that $\|u-w\| \leq\|u-\hat{w}\|$ and $\|\hat{u}-\hat{w}\| \leq\|\hat{u}-w\|$. Together with (2.9), (2.10) and (2.11) this implies that $\hat{w}=w$. With a similar argument we can show that $\varphi(\hat{u}, v)=w$ implies $\varphi(\hat{u}, \hat{v})=w$.

Lemma 2.24 Let assumption 2.1. Let $u, v \in A$ be such that $\varphi(u, v)=v$. Then $\varphi(w, v)=v$ for all $w \in[v, u, \rightarrow)$ with $[w, v] \subset A$.

Proof W.l.o.g. $u \neq v$. If $w \in[v, u]$, lemma 2.23 implies $\varphi(w, v)=v$. Now let $w \notin[v, u]$. First we assume $\|w-u\|<\|u-v\|$. Lemma 2.22 implies $\varphi(w, v) \in[w, v]$. By SP, $\|u-\varphi(w, v)\| \geq\|u-v\|$, hence $\varphi(w, v)=v$. Repeating this argument implies $\varphi(w, v)=v$ for all $w \in[v, u, \rightarrow)$.

Lemma 2.25 Let assumption 2.1 Then there exists an $\alpha \in(0, \pi)$ such that for al different $u, v, w \in \mathbf{R}^{m}$ with $\angle(u, v, w) \leq \alpha$ and $[v, w] \subset A$ we have that $\varphi(w, v)=v$ if $\varphi(u, v)=v$.

Proof Let $\|\cdot\|_{2}$ denote the Euclidean norm on $\mathbb{R}^{m}$. It is a well-known fact from elementary topology (cf. Kreyszig, 1978, p. 75) that there exist $\lambda_{1}, \lambda_{2}>0$ with $\frac{1}{\lambda_{1}}\|x\|_{2} \leq\|x\| \leq \frac{1}{\lambda_{2}}\|x\|_{2}$ for all $x \in \mathbb{R}^{m}$. Let $\alpha=\arctan \frac{\lambda_{2}}{\lambda_{1}}$. Now let different $u, v, w \in A$ be such that $L(u, v, w) \leq \alpha$ and $[v, w] \subset A$. Suppose $\varphi(u, v)=v$. (See figure 2.6.) Then

By definition of $\alpha$ we can take $y \in(v, w, \rightarrow)$ with $\|y-u\|_{2}<\frac{\lambda_{2}}{\lambda_{1}}\|v-u\|_{2}$.

$$
\|y-u\| \leq \frac{1}{\lambda_{2}}\|y-u\|_{2}<\frac{1}{\lambda_{2}} \frac{\lambda_{2}}{\lambda_{1}}\|v-u\|_{2} \leq\|v-u\| .
$$




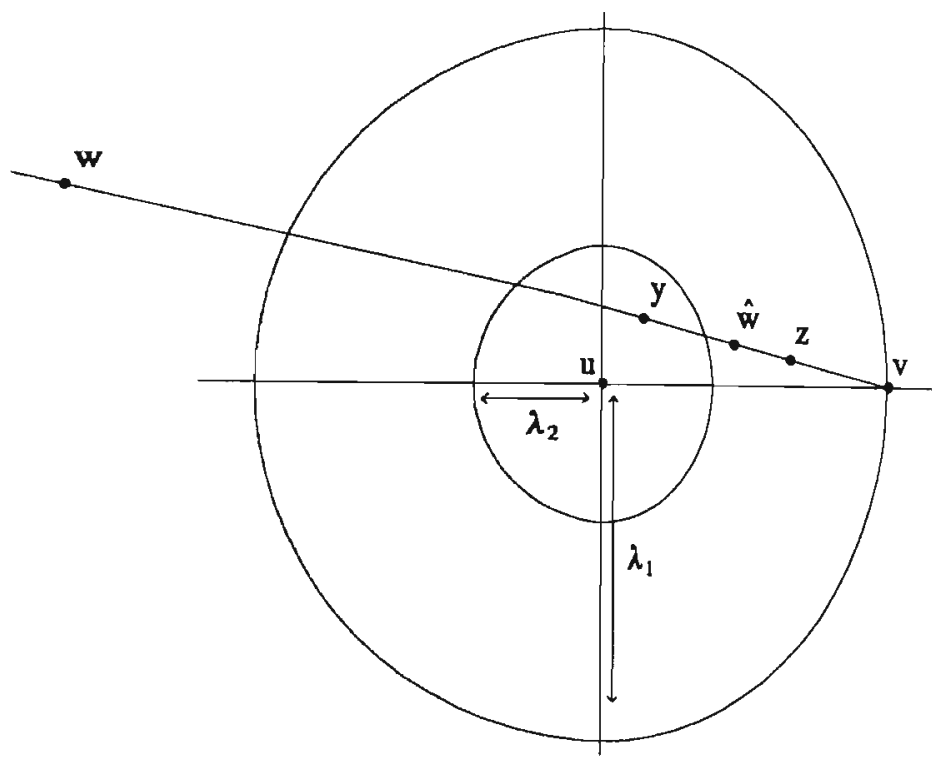

Figure 2.6: Proof of lemma 2.25

Let $\hat{w} \in(v, w) \cap(v, y)$. Let $z=\varphi(\hat{w}, v)$. Then by lemma $2.22, z \in[\hat{w}, v]$. Suppose $z \in[\hat{w}, v)$. Hence, $z-u \in(y-u, v-u)$. So by (2.12) and lemma A1 (i), $\|z-u\|<\|v-u\|$. This contradicts SP with respect to $\|\cdot\|$. Therefore, $z=\varphi(\hat{w}, v)=v$. Consequently, by lemma 2.24, $\varphi(w, v)=v$.

The following lemma follows from repeated application of lemma 2.25 (note that the openness of $A$ is essential).

Lemma 2.26 Let assumption 2.1. Let $u, v \in A$ be such that $u \neq v$ and $\varphi(u, v)=v$. Then $\varphi(x, v)=v$ for all $x \in A$ with $[v, x] \subset A$.

Similarly we can prove:

Lemma 2.27 Let assumption 2.1. Let $u, v \in A$ be such that $u \neq v$ and $\varphi(v, u)=v$. Then $\varphi(v, x)=v$ for all $x \in A$ with $[v, x] \subset A$.

Lemma 2.28 Let assumption 2.1. Then $\varphi(x, y) \in\{x, y\}$ for all $x, y \in A$.

Proof Let $x, y \in A, z=\varphi(x, y)$. Suppose $z \notin\{x, y\}$. Then by lemma 2.22, $z \in(x, y)$. By the openness of $A$ we can define $\hat{x} \in[x, z)$ and $\hat{y} \in[y, z)$ such that $[\hat{x}, \hat{y}] \subset A$. Positive association of $\varphi$ (see lemma 2.23) implies that 
$\varphi(\hat{x}, \hat{y})=z, \varphi(\hat{x}, z)=z$ and $\varphi(z, y)=z$. So by lemmas 2.26 and 2.27 , $\varphi(\hat{y}, z)=z$ and $\varphi(z, \hat{x})=z$. Hence by SP, $\|\varphi(\hat{y}, \hat{x})-\hat{x}\| \leq\|z-\hat{x}\|$ and $\|\varphi(\hat{y}, \hat{x})-\hat{y}\| \leq\|z-\hat{y}\|$. Therefore,

$$
\varphi(\hat{y}, \hat{x})=z .
$$

Let $u=\frac{1}{3} \hat{x}+\frac{2}{3} z$. Let $w=\varphi(\hat{x}, u)$. Then by SP, $\|w-u\| \leq\|w-z\|$. Hence $w \neq \hat{x}$. So by lemma 2.22

$$
w \in(\hat{x}, z) .
$$

Positive association of $\varphi$ implies that $\varphi(\hat{x}, w)=w$. Hence by lemma 2.26, $\varphi(\hat{y}, w)=w$. So by $(2.13)$ and SP, $\|z-\hat{x}\| \leq\|w-\hat{x}\|$, which contradicts (2.14). Consequently, $z \in\{x, y\}$.

Lemma 2.29 Let assumption 2.1. Let $x, y \in A$ with $\varphi(x, y)=x$. Then $\varphi(y, x)=y$.

Proof Assume w.l.o.g. that $x \neq y$. By the openness of $A$ we can define $z \in$ $(x, y)$ with $\|z-x\|<\|z-y\|$ and $[x, z] \subset A$. By lemma 2.28, $\varphi(z, y) \in\{z, y\}$. Furthermore, by SP, $\|z-\varphi(z, y)\| \leq\|z-\varphi(x, y)\|=\|z-x\|<\|z-y\|$. Hence $\varphi(z, y)=z$. So by lemma 2.27, $\varphi(z, x)=z$. Lemma 2.28 implies that $\varphi(y, x) \in\{x, y\}$. Also, by SP, $\|y-\varphi(y, x)\| \leq\|y-\varphi(z, x)\|=\|y-z\|<$ $\|y-x\|$. Consequently, $\varphi(y, x)=y$.

Similarly we can prove:

Lemma 2.30 Let assumption 2.1. Let $x, y \in A$ with $\varphi(x, y)=y$. Then $\varphi(y, x)=x$.

Lemma 2.31 Let assumption 2.1. Let $u, v \in A$ with $u \neq v$ and $\varphi(u, v)=v$. Then $\varphi(u, x)=u$ for all $x \in A$.

Proof Let $x \in A$. Suppose $\varphi(u, x) \neq u$. Then by lemma $2.28, \varphi(u, x)=x$. Hence, $x \neq u$. By the openness of $A$ we can define $\hat{x} \in[x, u)$ such that $[u, \hat{x}] \subset A$. Lemma 2.30 implies that $\varphi(x, u)=u$. So by positive association (see lemma 2.23), $\varphi(\hat{x}, u)=u$. Hence by lemma 2.30, $\varphi(u, \hat{x})=\hat{x}$. On the other hand, by lemma $2.26, \varphi(u, \hat{x})=u$. Therefore, we have a contradiction. Consequently, $\varphi(u, x)=u$.

Theorem 2.2 Let $A$ be a nonempty open subset of $\mathbf{R}^{m}, m \geq 2$. Let $\varphi$ : $A^{2} \rightarrow A$ satisfy strong Pareto optimality and strategy-proofness with respect to a norm on $\mathbb{R}^{m}$. Then $\varphi$ is dictatorial. 
Proof Let $u, v \in A$ with $u \neq v$. Suppose $\varphi(u, v)=u$. Then by lemma 2.31, $\varphi(u, x)=u$ for all $x \in A$. So by lemma 2.29, $\varphi(x, u)=x$ for all $x \in A$. Hence by lemma $2.29, \varphi(x, y)=x$ for all $x, y \in A$. Consequently, $\varphi$ is dictatorial. Now assume $\varphi(u, v) \neq u$. Then by lemma $2.28, \varphi(u, v)=v$. Similarly as above, it follows that $\varphi$ is dictatorial.

So for $m \geq 2$, all the schemes of an $m$-dimensional 2-person location problem which satisfy SPO and SP with respect to a norm are dictatorial. Therefore, there are no schemes to such a problem which satisfy AN.

The following corollary is a direct consequence of theorem 2.1 and proposition 2.1 .

Corollary 2.6 Let $A$ be a nonempty open subset of $\mathbf{R}^{m}, m \geq 2$. Let $\varphi$ : $A^{2} \rightarrow A$ satisfy (weak) Pareto optimality and strategy-proofness with respect to a strictly convex norm on $\mathbb{R}^{m}$. Then $\varphi$ is dictatorial.

So for $m \geq 2$, all voting schemes of an $m$-dimensional 2-person location problem which satisfy $\mathrm{PO}$ and SP with respect to a strictly convex norm are dictatorial.

We now use theorem 2.2 to obtain results for the case where an arbitrary even number of agents is considered. Lemma 2.32 states that such a voting scheme which also satisfies anonymity, must satisfy a strong majority rule, i.e., if at least half of the agents reports the same point, the compromise point must be this point.

Lemoma 2.32 Let $A$ be a nonempty open subset of $\mathbf{R}^{m}, m \geq 2$. Let $\varphi$ : $A^{N} \rightarrow A$ satisfy $A N$ and $S P O$ and $S P$ with respect to a norm on $\mathbf{R}^{m}$. Let $S \subset N$. Let $u, v \in A$ with $u \neq v$. Then $\varphi\left(\left\langle u^{S}, v^{N \backslash S}\right\rangle\right)=u$ if and only if $|S| \geq \frac{n}{2}$.

Proof Let $\psi: A^{2} \rightarrow A$ be defined by $\psi(x, y)=\varphi\left(\left\langle x^{S}, y^{N \backslash S}\right\rangle\right)$ for all $x, y \in A$. Then by lemma $1.2 \psi$ is strategy-proof with respect to $\|\cdot\|$. It is easy to verify that $\psi$ also satisfies $\mathrm{PO}$ with respect to $\|\cdot\|$. So by theorem $2.2, \psi(x, y)=x$ for all $x, y \in A$ or $\psi(x, y)=y$ for all $x, y \in A$. Hence

$$
\begin{aligned}
\varphi\left(\left\langle x^{S}, y^{N \backslash S}\right\rangle\right)= & x \text { for all } x, y \in A \text { or } \\
& \varphi\left(\left\langle x^{S}, y^{N \backslash S}\right\rangle\right)=y \text { for all } x, y \in A .
\end{aligned}
$$

Suppose $|S| \geq \frac{n}{2}$ and $\varphi\left(\left\langle u^{S}, v^{N \backslash S}\right\rangle\right) \neq u$. Then by (2.15),

$$
\varphi\left(\left\langle u^{S}, v^{N \backslash S}\right\rangle\right)=v \text { and } \varphi\left(\left\langle u^{S}, v^{N \backslash S}\right\rangle\right)=u .
$$


Let $T \subset S$ with $|T|=|N \backslash S|$.

By $(2.16), \varphi\left(\left\langle u^{T}, v^{N \backslash S}, u^{S \backslash T}\right\rangle\right)=v$. Hence by AN, $\varphi\left(\left\langle v^{T}, u^{N \backslash S}, u^{S \backslash T}\right\rangle\right)=$ $v$. So by confirmatoriness (see lemma 1.4), $\varphi\left(\left\langle v^{T}, u^{N \backslash S}, v^{S \backslash T}\right\rangle\right)=v$. So $\varphi\left(\left\langle v^{S}, u^{N \backslash S}\right\rangle\right)=v$. This contradicts (2.16). Consequently, $|S| \geq \frac{n}{2}$ implies that $\varphi\left(\left\langle u^{S}, v^{N \backslash S}\right\rangle\right)=u$. Similarly we can prove that $|S|<\frac{\mathbf{n}}{2}$ implies that $\varphi\left(\left\langle u^{S}, v^{N \backslash S}\right\rangle\right)=v$.

The following theorem states that if the number of agents is even, there are no voting schemes on $A \subset \mathbf{R}^{m}$ with $m \geq 2$ which satisfy anonymity, and strong Pareto optimality and strategy-proofness with respect to a norm.

Theorem 2.3 Let $A$ be a nonempty open subset of $\mathbb{R}^{m}, m \geq 2$. Let $n$ be even. Let $\|\cdot\|$ be a norm on $\mathbf{R}^{m}$. Then there is no $\varphi: A^{N} \rightarrow A$ satisfying $A N$, and $S P O$ and $S P$ with respect to $\|\cdot\|$.

Proof Suppose, to the contrary, that such a $\varphi$ exists. Let $S \subset N$ with $|S|=\frac{1}{2} n$. Let $u, v \in A$ with $u \neq v$. Since $|S| \geq \frac{1}{2} n$ and $|N \backslash S| \geq \frac{1}{2} n$, lemma 2.32 implies that $u=\varphi\left(\left\langle u^{S}, v^{N \backslash S}\right\rangle\right)=v$, a contradiction.

Since by proposition 2.1 with respect to a strictly convex norm WPO, PO and SPO are equivalent, theorem 2.2 implies:

Corollary 2.7 Let $A$ be a nonempty open subset of $\mathbb{R}^{m}, m \geq 2$. Let $n$ be even. Let $\|\cdot\|$ be a strictly convex norm on $\mathbb{R}^{m}$. Then there is no $\varphi: A^{N} \rightarrow A$ satisfying $A N$, and $P O$ (or WPO) and $S P$ with respect to $\|\cdot\|$.

In literature no attention seems to be paid to the property SPO. Many authors however consider PO, for instance Kim and Roush (1984). Their corollary 38 implies that for $m \geq 2$ and an even number of agents, there are no voting schemes $\varphi:\left(\mathbf{R}^{m}\right)^{N} \rightarrow \mathbf{R}^{m}$ satisfying $\mathrm{AN}$ and $\mathrm{PO}$ and $\mathrm{SP}$ with respect to the Euclidean norm which are componentwise continuous.

Corollary 2.7 is stronger in four ways:

1.) We do not need the requirement that $\varphi$ must be componentwise continuous.

2) This result is valid with respect to all strictly convex norms whereas Kim and Roush only consider the Euclidean norm (this norm is strictly convex, cf. lemma 3.1).

3) We allow the set of alternatives to be a nonempty open subset of $\mathbf{R}^{m}$, whereas Kim and Roush only consider the case $A=\mathbb{R}^{m}$. 
4) PO can be relaxed to WPO.

\subsection{Three person location problems in $\mathbb{R}^{m}$}

In this section we study $\varphi:\left(\mathbf{R}^{m}\right)^{N} \rightarrow \mathbf{R}^{m}$ with $m \geq 2$ and $n=3$, where $\varphi$ is assumed to satisfy SP and PO with respect to a strictly convex norm $\mid \cdot \|$. The results of this section will be used in section 2.6, where we restrict ourselves to the less general case $m=2$. Finally we will show how this problem is related to the more general location problem with $n \geq 3$. Throughout this section we use notations like $\varphi(u, v, w)$ instead of $\varphi(p)$, where $p \in\left(\mathbf{R}^{m}\right)^{N}$, $p(1)=u, p(2)=v$ and $p(3)=w$. The following assumption will be used in lemmas 2.33-2.40:

Assumption $2.2 m \geq 2, n=3$ and $\varphi:\left(\mathbf{R}^{m}\right)^{N} \rightarrow \mathbb{R}^{m}$ satisfies $S P$ and $P O$ with respect to a strictly convex norm $\|\cdot\|$.

A nondictatorial scheme must satisfy the majority rule: if two of the agents (which is the majority) both report the same points, this will be the compromise point:

Lemma 2.33 Let assumption 2.2. If $\varphi$ is not dictatorial, then $\varphi(x, x, y)=$ $\varphi(x, y, x)=\varphi(y, x, x)=x$ for all $x, y \in \mathbf{R}^{m}$.

Proof Let $\psi:\left(\mathbf{R}^{m}\right)^{2} \rightarrow \mathbf{R}^{m}$ be such that $\psi(x, y)=\varphi(x, x, y)$. Then by lemma $1.2, \psi$ satisfies SP with respect to $\|\cdot\|$. Since $\psi$ also satisfies PO, corollary 2.2 implies that $\psi(x, y)=x$ for all $x, y \in \mathbf{R}^{m}$ or $\psi(x, y)=y$ for all $x, y \in \mathbf{R}^{m}$. Hence, $\varphi(x, x, y)=x$ for all $x, y \in \mathbf{R}^{m}$ or $\varphi(x, x, y)=y$ for all $x, y \in \mathbf{R}^{m}$. Consequently, if $\varphi$ is not dictatorial, $\varphi(x, x, y)=x$ for all $x, y \in \mathbb{R}^{m}$. Similarly we can prove that $\varphi(x, y, x)=\varphi(y, x, x)=x$ for all $x, y \in \mathbf{R}^{m}$ if $\varphi$ is not dictatorial.

If the three reported points are collinear, a nondictatorial scherne assigns the middle of these points:

Lemma 2.34 Let assumption 2.2. If $\varphi$ is not dictatorial, then $\varphi(u, v, w)$ $=u$ for all $u, v, w \in \mathbf{R}^{m}$ with $u \in[v, w]$.

Proof Let $u, v, w \in \mathbf{R}^{m}$ with $u \in[v, w]$. Let $y=\varphi(u, v, w)$. Then lemma 2.12 implies that $y \in[v, w]$. Also, by lemma 2.33, $\varphi(u, u, w)=\varphi(u, v, u)=u$. So by SP, ||$w-y\|\leq\| w-u \|$ and $\|v-y\| \leq\|v-u\|$. Consequently, $y=u$. 
A nondictatorial scheme assigns a point to a profile such that this point is closer to each reported point than each of the two other reported points. It is even stronger:

Lemma 2.35 Let assumption 2.2. Let $\varphi$ be nondictatorial. Let $a, b, c$, $w \in \mathbb{R}^{m}$ with $w=\varphi(a, b, c)$. Then $\|a-w\|<\|a-x\|$ for all $x \in(w, b, \rightarrow)$.

Proof W.l.o.g. assume $w \neq b$. Confirmatoriness (lemma 1.4) implies that $\varphi(a, b, w)=w$. Since $\varphi$ is nondictatorial, we have by lemma 2.34 that $\varphi(\tilde{x}, b, w)=\tilde{x}$ for all $\tilde{x} \in[b, w]$. So by SP,

$$
\|a-w\| \leq\|a-\tilde{x}\| \text { for all } \tilde{x} \in[b, w] .
$$

Let $x \in(w, b, \rightarrow)$. Suppose $\|a-x\| \leq\|a-w\|$. Then by lemma 2.1. (iii) $\|a-\tilde{x}\|<\|a-w\|$ for all $\tilde{x} \in(x, w)$. This contradicts (2.17). Consequently, $\|a-w\|<\|a-x\|$ for $x \in(w, b, \rightarrow)$.

Lemma 2.36 states that Pareto optimality and strategy-proofness with respect to a strictly convex norm imply componentwise continuity in the three person case.

Lemma 2.36 Let assumption 2.2. Then $\varphi$ is componentwise continuous.

Proof W.lo.g. assume that $\varphi$ is nondictatorial (otherwise, the lemma is trivial). We will prove that $\varphi$ is continuous in the first component. The proof that $\varphi$ is continuous in the other components, is similar.

Let $x, y, z, u, x^{i} \in \mathbf{R}^{m}(i \in \mathbb{N})$ be such that $\varphi(x, y, z)=u, x^{i} \rightarrow x$. Suppose $\varphi\left(x^{i}, y, z\right) \nrightarrow u$. Lemma 2.16 implies that

$$
\left\|x-\varphi\left(x^{i}, y, z\right)\right\| \rightarrow\|x-u\| .
$$

Hence, we may assume that $\left\|x-\varphi\left(x^{i}, y, z\right)\right\| \leq\|x-u\|+1$ for all $i \in \mathbb{N}$. Since $\left\{\tilde{x} \in \mathbf{R}^{m}:\|\tilde{x}-x\| \leq\|x-u\|+1\right\}$ is a compact set, we may assume that $\varphi\left(x^{i}, y, z\right)$ converges, say to $v$. Since $\varphi\left(x^{i}, y, z\right) \nrightarrow u, u \neq v$. By (2.18),

$$
\|x-u\|=\|x-v\| .
$$

Suppose that $\|y-v\|<\|y-u\|$. Then by SP, $\left\|v-\varphi\left(x^{i}, v, z\right)\right\| \leq \| v-$ $\varphi\left(x^{i}, y, z\right) \|$ for all $i \in \mathbf{N}$. Hence, $\varphi\left(x^{i}, v, z\right) \rightarrow v$. Let $w=\varphi(x, v, z)$. By $\mathrm{SP},\|y-u\| \leq\|y-w\|$, so by our assumption, $\|y-v\|<\|y-w\|$. Hence, 
$v \neq w$. By a similar argument as we used to obtain (2.19), we find that $\|x-v||=|| x-w\|$. On the other hand, by lemma 2.35, $\|x-w\|<\|x-v\|$. So we have a contradiction.

Consequently, $\|y-u\| \leq\|y-v\|$. Similarly we can prove that $\| z-$ $u\|\leq\| z-v \|$. Let $d=\frac{1}{2} u+\frac{1}{2} v$. Then by (2.19) and lemma 2.1 (iii), $x-d\|<\| x-v\|\| y-,d\|<\| y-v \|$ and $\|z-d\|<\|z-v\|$. Since $x^{i} \rightarrow x$ and $\varphi\left(x^{i}, y, z\right) \rightarrow v$, we can take $i \in \mathbb{N}$ such that $\left\|x^{i}-d\right\|<\left\|x^{i}-\varphi\left(x^{i}, y, z\right)\right\|$, $\|y-d\|<\left\|y-\varphi\left(x^{i}, y, z\right)\right\|$ and $\|z-d\|<\left\|z-\varphi\left(x^{i}, y, z\right)\right\|$. This contradicts $\mathrm{PO}$.

Next two lemmas establish a property which extends positive associativity (as defined in section 2.2): also moving in the direction opposite to the compromise point does not change this compromise point.

Lemma 2.37 Let assumption 2.2. Let $a, b, c \in \mathbf{R}^{m}$ be such that $\varphi(a, b$, $c)=a$. Then $\varphi(a, d, c)=a$ for all $d \in[a, b, \rightarrow)$.

Proof If $a=b$, then the desired result is trivial. Next assume $a \neq b$. If $\varphi$ is dictatorial, again we are done. Assume w.l.o.g. that $\varphi$ is nondictatorial. In view of positive association (lemma 2.13) we may assume that $d \notin[a, b]$. Lemma 2.35 implies that

$$
\|c-a\|<\|c-b\| .
$$

Let $C=\left\{x \in \mathbb{R}^{m}: \varphi(a, x, c)=x\right\}$. By SP and lemma 2.33 we have that $\|x-c\| \leq\|a-c\|$ for all $x \in C$. Let $B=\left\{\tilde{x} \in \mathbf{R}^{m}:\|\tilde{x}-c\| \leq\|a-c\|\right\}$. Then $C \subset B$ and $B$ is a convex set. By (2.20), $b \notin B$. Let - denote the standard inner product on $\mathbf{R}^{m}$. By a separation theorem of convex analysis, (cf. Rockafellar, 1970, p. 98) we can take $u \in \mathbf{R}^{m} \backslash\{0\}$ with $u \cdot x<u \cdot b$ for all $x \in B$. Let $D=\left\{\tilde{x} \in \mathbf{R}^{m}: u \cdot \tilde{x}=u \cdot d\right\}$. Let $f: B \rightarrow \mathbf{R}^{m}$ be defined by $f(x)=b+\frac{u \cdot(d-b)}{u \cdot(b-x)}(b-x)$ for all $x \in B$. Since $u \cdot f(x)=u \cdot d$ for all $x \in B, f(B) \subset D$. Since $B$ is compact and $f$ is continuous on $B, f(B)$ is compact. So conv $f(B)$ is also compact. Let $g: \operatorname{conv} f(B) \rightarrow \mathbf{R}^{m}$ be defined by $g(x)=f(\varphi(a, x, c))$ for $x \in \operatorname{conv} f(B)$. Since by confirmatoriness (see lemma 1.4), $\varphi(a, x, c) \in C$ for all $x \in \mathbf{R}^{m}$ and $C \subset B$, we have that $g$ maps conv $f(B)$ into conv $f(B)$. Since $f$ is continuous and $\varphi$ is componentwise continuous, $g$ is continuous. Since conv $f(B)$ is also nonempty, we have by the fixed point theorem of Brouwer that $g$ has a fixed point $\hat{x} \in \operatorname{conv} f(B)$, i.e. $g(\hat{x})=\hat{x}$. So $f(\varphi(a, \hat{x}, c))=\hat{x}$. Hence, $b \in(\hat{x}, \varphi(a, \hat{x}, c))$. So by positive association (see lemma 2.13), $\varphi(a, b, c)=\varphi(a, \hat{x}, c)$. Hence, $\varphi(a, \hat{x}, c)=a$. So $d=f(a)=f(\varphi(a, \hat{x}, b))=\hat{x}$. Consequently, $\varphi(a, d, c)=a$. 
Lemma 2.38 Let assumption 2.2. Let $a, b, c, w \in \mathbf{R}^{m}$ be such that $\varphi(a, b, c)=$ w. Let $d \in[w, a, \rightarrow)$. Then $\varphi(d, b, c)=w$.

Proof Let $v=\varphi(d, b, c)$. Suppose $v \neq w$. Confirmatoriness (lemma 1.4) implies that $\varphi(w, b, c)=\varphi(a, w, c)=\varphi(a, b, w)=w$. So by lemma 2.37, $\varphi(w, b, c)=\varphi(d, w, c)=\varphi(d, b, w)=w$. Hence, by SP,

$$
\|x-v\| \leq\|x-w\| \text { for all } x \in\{d, b, c\} .
$$

In view of positive association (lemma 2.13) we assume w.l.o.g. that $d \notin$ $[a, w]$. Let $\lambda=\| \frac{a-w \|}{\| d-w \mid}$. Then $\lambda \in(0,1)$. Let $u=w+\lambda(v-w)$. Then $\|a-u\|=\|a-w-\lambda(v-w)\|=\lambda\left\|\frac{1}{\lambda}(a-w)-(v-w)\right\|=\lambda\|d-w-(v-w)\|=$ $\lambda\|d-v\| \leq \lambda|| d-w\|=\| a-w \|$. Furthermore, by lemma 2.1 (iii) and (2.21), $\|b-u\|<\|b-w\|$ and $\|c-u\|<\|c-w\|$. So, with PO, we have a contradiction. Consequently, $v=w$.

Dependent on the compromise point we can exclude many points in $\mathbb{R}^{m}$ from the effectivity set of an agent, i.e., the set of points which that agent can enforce to be the compromise point, given the choices of the other agents:

Lemma 2.39 Let assumption 2.2. Let $a, b, c, u, v, w \in \mathbf{R}^{m}$ be such that $\varphi(a, b, c)=u$ and $\varphi(v, b, c)=w$. Then $\|a-u\|<\|a-x\|$ for all $x \in$ $(u, w, \rightarrow)$.

Proof W.l.o.g. $u \neq w$. Let $x \in(u, w, \rightarrow)$. Let $y \in(u, x)$. Let $\lambda=\|y-u\|$. Let $d=u+\lambda(a-u)$. Then by lemma $2.38 \varphi(d, b, c)=u$. Hence by SP, $\|d-u\| \leq\|d-w\|$. So $\|a-u\|=\frac{1}{\lambda}|| d-u\left\|\leq \frac{1}{\lambda}\right\| d-w\left\|=\frac{1}{\lambda}\right\| d-u+u-w \|=$ $\left\|a-u+\frac{1}{\lambda}(u-w)\right\|=\left\|a-\left(u+\frac{\|y-u\|}{\| w-u \mid}(w-u)\right)\right\|=\|a-y\|$. So by lemma 2.1 (iii), $\|a-u\|<\|a-x\|$, because $y \in(u, x)$.

If the compromise point is between two of the reported points for a profile for which the reported points are not collinear, $\|\cdot\|$-orthogonality of two directions can be established:

Lemma 2.40 Let assumption 2.2. Let $a, b, c, d \in \mathbf{R}^{m}$ be such that $\varphi(a, b, c)=$ $d \in(b, c)$. Then $b-c$ and $a-c$ are $\|\cdot\|$-orthogonal.

Proof W.l.o.g. assume that $a \neq d$. Since $d \notin\{a, b, c\}, \varphi$ is nondictatorial. Lemma 2.35 implies that $\|a-d\|<\|a-x\|$ for all $x \in(d, b, \rightarrow) \cup(d, c, \rightarrow)$. So $\|a-d\| \leq\|a-d+d-x\|$ for al] $x \in(\leftarrow, b, c, \rightarrow)$. Hence,

$$
\|a-d\| \leq\|a-d+\lambda(b-c)\| \text { for all } \lambda \in \mathbf{R} \text {. }
$$


Also by lemma 2.35, $\|b-d\|<\|b-x\|$ for all $x \in(d, a, \rightarrow)$. So, $\|b-d\| \leq$ $\|b-d+\lambda(a-d)\|$ for all $\lambda \in[0, \infty)$. Hence,

$$
\|\boldsymbol{b}-\boldsymbol{c}\| \leq\|b-c+\lambda(a-d)\| \text { for all } \lambda \in[0, \infty)
$$

Similarly, $\|c-d\| \leq\|c-d+\lambda(a-d)\|$ for all $\lambda \in[0, \infty)$. Hence, $\|b-c\| \leq$ $\|b-c-\lambda(a-d)\|$ for all $\lambda \in[0, \infty)$. Together with (2.23) this implies that $\|b-c\| \leq\|b-c+\lambda(a-d)\|$ for all $\lambda \in \mathbf{R}$. This implies together with (2.22) the $\|\cdot\|$-orthogonality of $b-c$ and $a-c$ in the sense of Birkhoff. Since for two vectors $\|\cdot\|$-orthogonality and $\|\cdot\|$-orthogonality in the sense of Birkhoff are equivalent, we are done.

Given an $n$-person voting scheme, with $n>3$ and a partition of $N$ in 3 parts, a 3 person voting scheme can be constructed by considering each of the parts as one agent. Under some conditions anonymity, strategy-proofness and Pareto optimality of the original scheme imply the same properties for the reduced scheme.

Lemma 2.41 Let $m \geq 2$. Let $\varphi:\left(\mathbb{R}^{m}\right)^{N} \rightarrow \mathbf{R}^{m}$ satisfy $A N$ and $P O$ and $S P$ with respect to a strictly convex norm $\|\cdot\|$. Let nonempty, disjoint $R, S, T \subset N$ be such that $R \cup S \cup T=N,|R|<\frac{n}{2},|S|<\frac{n}{2}$ and $|T|<\frac{n}{2}$. Let $\psi:\left(\mathbf{R}^{m}\right)^{3} \rightarrow \mathbf{R}^{m}$ be defined by $\psi(x, y, z)=\varphi\left(\left\langle x^{R}, y^{S}, z^{T}\right\rangle\right)$ for all $x, y, z \in \mathbb{R}^{m}$. Then $\psi$ satisfies $A N$ and $P O$ and $S P$ with respect to $\|\cdot\|$.

Proof The Pareto optimality of $\psi$ is evident. The strategy-proofness of $\psi$ follows from lemma 1.2. Suppose $\psi$ is dictatorial. Then we may assume that $\psi(x, y, z)=x$ for all $x, y, z \in \mathbf{R}^{m}$. Let $\hat{x}, \hat{y} \in \mathbf{R}^{m}$ with $\hat{x} \neq \hat{y}$. Hence $\psi(\hat{x}, \hat{y}, \hat{y})=\hat{x}$. So $\varphi\left(\left\langle\hat{x}^{R}, \hat{y}^{S \cup T}\right\rangle\right)=\hat{x}$. Therefore by lemma $2.32,|R| \geq$ $|S \cup T|$. So $|R| \geq \frac{1}{2} n$, which contradicts our assumptions. Consequently, $\psi$ is nondictatorial.

Now suppose $\psi$ is not anonymous. Then w.l.o.g. there are $a, b, c, u, v \in$ $\mathbf{R}^{m}$ with $\psi(a, b, c)=u$ and $\psi(b, a, c)=v \neq u$. Suppose $\|a-u\|<\|a-v\|$. By confirmatoriness (see lemma 1.4), $\psi(u, b, c)=u$. Let $w=\psi(b, u, c)$. Then by SP, $\|a-v\| \leq\|a-w\|$. So $u \neq w$. Hence, by lemma 2.35, $\|c-w\|<\|c-u\|$. Confirmatoriness implies that $\psi(b, u, w)=w$. Let $\tilde{w}=\psi(u, b, w)$. Then by SP, $\tilde{w} \neq w$. So lemma 2.35 implies that:

$$
\|b-\tilde{w}\|<\|b-w\| \text { and }\|u-\tilde{w}\|<\|u-w\| .
$$

By definition, $\varphi\left(\left\langle u^{R}, b^{S}, w^{T}\right\rangle\right)=w$ and $\varphi\left(\left\langle b^{R}, u^{S}, w^{T}\right\rangle\right)=\tilde{w}$. By lemma 1.1 $\varphi$ satisfies ISP. If $|R| \leq|S|$ we have by ISP and AN that $\|b-w\| \leq\|b-\tilde{w}\|$, 
which contradicts (2.24). Similarly, if $|S| \leq|R|$, we have that ||$u-w|| \leq$ $\|u-\tilde{w}\|$, which also contradicts (2.24). Consequently, $\|a-u\| \geq\|a-v\|$. Similarly, we can prove that $\|a-v\| \geq\|a-u\|,\|b-u\| \geq\|b-v\|$ and $\|b-v\| \geq\|b-u\|$. Hence, $\|a-u\|=\|a-v\|$ and $\|b-u\|=\|b-v\|$. If $\|c-u\| \leq\|c-v\|$ then by lemma 1.1, $\left\|x-\left(\frac{1}{2} u+\frac{1}{2} v\right)\right\|<\|x-v\|$ for all $x \in\{a, b, c\}$, contradicting the Pareto optimality of $\varphi$. Similarly, if $\|c-v\| \leq\|c-u\|$, then $\left\|x-\left(\frac{1}{2} u+\frac{1}{2} v\right)\right\|$ for all $x \in\{a, b, c\}$, which also contradicts PO. Consequently, $\varphi$ is anony mous.

\subsection{Three person location problems in $\mathbb{R}^{2}$}

In the previous section we obtained some results for three person location problems in $\mathbf{R}^{m}$. We now restrict ourselves to the case $m=2$, and present a complete characterization of the schemes to this problem which are also anonymous. It is unclear till sofar which lemmas of this section can be generalized to arbitrary dimension $m \geq 2$. Throughout this section we will use:

Assumption $2.3 n=3, m=2$, and $\varphi:\left(\mathbf{R}^{2}\right)^{N} \rightarrow \mathbf{R}^{2}$ satisfies $P O$ and $S P$ with respect to a strictly convex norm $\|\cdot\|$.

This section is devoted to a proof of theorem 2.3. This theorem asserts that all voting schemes satisfying assumption 2.2 and anonymity must be coordinatewise median schemes with respect to some $\|\cdot\|$-orthogonal basis of $\mathbf{R}^{2}$ without constant points. For the structure of this section and intuitive meanings of several lemmas we refer to the texts between the lemmas.

If a point $w$ in the convex hull of three other points dominates these three points in a certain way, this will be the compromise point assigned to the profile which has these three points as components:

Lemma 2.42 Let assumption 2.9. Let $a, b, c \in \mathbf{R}^{2}$ be noncollinear and $w \in \Delta(a, b, c)$ such that $\varphi(a, b, w)=\varphi(a, w, c)=\varphi(w, b, c)=w$. Then $\varphi(a, b, c)=w$.

Proof Let $v=\varphi(a, b, c)$. Suppose $v \neq w$. Then by lemma 2.39,

$$
\|u-v\|<\|u-w\| \text { for all } u \in\{a, b, c\} .
$$

Since $w \in \Delta(a, b, c)$, we can take $r \in[a, b] \cup[b, c] \cup[c, a]$ such that $w \in[r, v)$. W.l.o.g. assume that $r \in[a, b]$. Lemma 2.1 (iv) implies that $\|a-w\|<\|a-r\|$ and $\|b-w\|<|| b-r||$. Hence, $\|a-w\|+\|b-w\|<\|a-r\|+\|b-r\|=\|a-b\|$, 
which contradicts the triangular inequality for norms.

Lemmas 2.43 and 2.44 are only used to prove lemma 2.46 . The first two lemmas consider the situation which is depicted in figure 2.7: the nondictatorial scheme $\varphi$ is such that $\varphi(a, b, c)=a$ and $\varphi(u, b, c)=v \neq u$. Lemma 2.43 states that if we replace $b$ by $a$ point $\tilde{b}$ on the half-line $[u, \tilde{b}, \rightarrow)$ and $c$ by $a$ point $\tilde{c}$ on the half-line $[a, c, \rightarrow)$, the corresponding compromise point remains on the half-line $[u, v, \rightarrow)$.

Lemma 2.43 Let assumption 2.3. Let $\varphi$ be nondictatorial. Let $a, b, c \in \mathbf{R}^{2}$ be such that $\varphi(a, b, c)=a$. Let $u \in(a, b)$ with $v=\varphi(u, b, c) \neq u$. Let $\tilde{b} \in[u, b, \rightarrow), \tilde{c} \in[a, c, \rightarrow)$. Then $\varphi(u, \tilde{b}, \tilde{c}) \in[u, v, \rightarrow)$.

Proof By lemma 2.34, $a, b$ and $c$ are noncollinear. Let $w=\varphi(u, \tilde{b}, \tilde{c})$. Suppose $w \notin[u, v, \rightarrow]$. Lemma 2.38 implies:

$$
\varphi(a, \tilde{b}, \tilde{c})=a
$$

By PO and theorem 2.1, $v \in \Delta(u, b, c)$. If $v \in[b, u)$, then by lemma 2.38, $\varphi(a, b, c)=v$, so $v=a=u$, a contradiction. Hence, $v \notin[b, u)$. Similarly, $w \notin[\tilde{b}, u)$. W.l.o.g. assume $\angle(a, u, v)<L(a, u, w)$ (if not, we can use a similar argument). (See figure 2.7.)

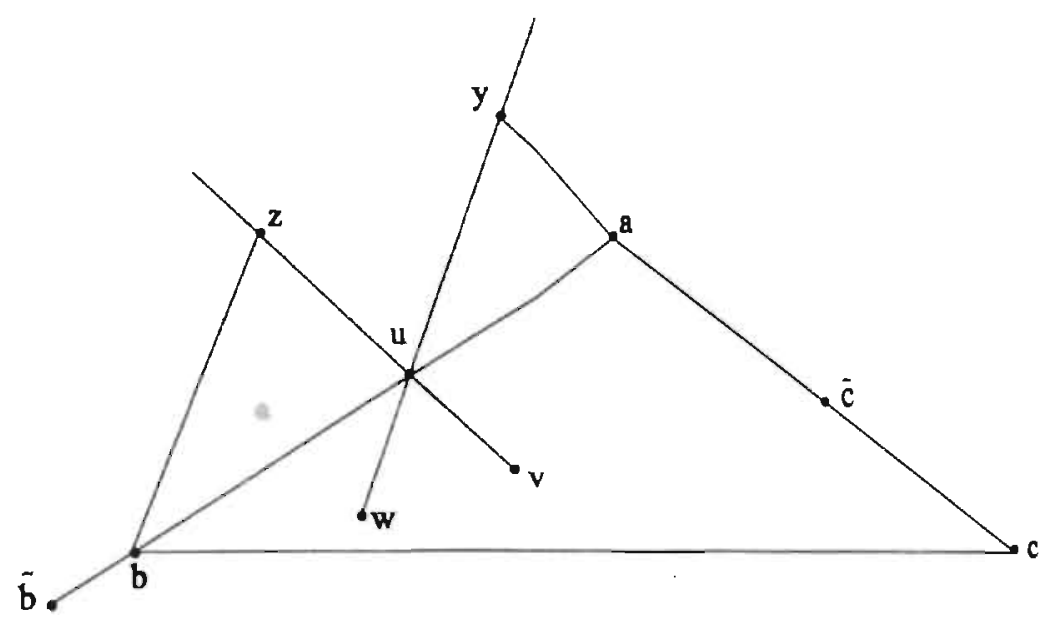

Figure 2.7: Proof of lemmo 2. 49 
Let $y \in[w, u, \rightarrow)$ be such that $(y, a) / /(u, v)$. By lemma 2.38, $\varphi(y, \tilde{b}, \tilde{c})=$ $w$. So by lemma 2.39 and $(2.26),\|y-w\|<\|y-a\|$. Hence,

$$
\|y-u\|<\|y-a\|
$$

Let $z \in[v, u, \rightarrow]$ be such that $(z, b) / /(u, w)$. By lemma $2.38, \varphi(z, b, c)=v$. So by lemma 2.35, $\|z-v\|<\|z-b\|$. Hence,

$$
\|z-u\|<\|z-b\| .
$$

Let $\lambda \in(0, \infty)$ be such that $z-b=\lambda(y-u)$. Then $z-u=\lambda(y-a)$. So (2.28) implies $\|y-a\|<\|y-u\|$, which contradicts (2.27).

Lemma 2.44 states that we can take $\tilde{b} \neq u$ and $\tilde{c} \neq a$ (here called $y$ and $z$ ) such that $\varphi(u, \tilde{b}, \tilde{c})$ is on the line segment between $\tilde{b}$ and $\tilde{c}$ (cf. figure 2.8).

Lemma 2.44 Let assumption 2.9. Let $\varphi$ be nondictatorial. Let $a, b, c \in \mathbb{R}^{2}$ be such that $\varphi(a, b, c)=a$. Let $u \in(a, b)$ be such that $\varphi(u, b, c)=w \neq$ u. Then there are $y \in(u, b, \rightarrow), z \in(a, c, \rightarrow)$ such that $w \in(y, z)$ and $\varphi(u, y, z)=w$.

Proof (See figure 2.8.) Let $z(\lambda)=a+\lambda(c-a), \lambda \in[0,1]$. Let $f:[0,1] \rightarrow \mathbb{R}^{2}$ be defined by $f(\lambda)=\varphi(u, b, z(\lambda))$. Since $\varphi$ is componentwise continuous (see lemma 2.36), $f$ is continuous on $[0,1] . f(0)=u$ because of lemma 2.34; $f(1)=w$. Furthermore, by lemma $2.43, f(\lambda) \in[u, w, \rightarrow)$ for all $\lambda \in[0,1]$. Let $\lambda_{\min }=\inf \{\lambda \in[0,1]: f(\lambda)=w\}$. Because of the continuity of $f$, $f\left(\lambda_{\min }\right)=w$. Hence, $\lambda_{\min }>0$. Let $z=z\left(\lambda_{\min }\right)$. Let $y(\mu)=u+\mu(b-u)$, $\mu \in[0,1]$. Let $g:[0,1] \rightarrow \mathbf{R}^{2}$ be defined by $g(\mu)=\varphi(u, y(\mu), z)$. Since $\varphi$ is componentwise continuous (see lemma 2.36), $g$ is continuous on $[0,1]$. $g(0)=u$ because of lemma $2.33 ; g(1)=w$. Furthermore, by lemma 2.43, $g(\mu) \in[u, w, \rightarrow)$ for all $\mu \in[0,1]$. Let $\mu_{\min }=\inf \{\mu \in[0,1]: g(\mu)=w\}$. Because of the continuity of $g, g\left(\mu_{\min }\right)=w$. Hence, $\mu_{\min }>0$. Let $y=$ $y\left(\lambda_{\min }\right)$. Then $[y, z] \cap[u, w, \rightarrow] \neq \emptyset$. Let $d \in[y, z] \cap[u, w, \rightarrow)$. If $d \in[u, w)$ then $w \notin \Delta(u, y, z)$, which contradicts theorem 2.1. Suppose $d \neq w$. Then lemma 2.1 (v) implies that $\|y-w\|+\|z-w\|>\|y-z\|=\|y-d\|+\|z-d\|$. W.l.o.g. assume $\|y-d\|<\|y-w\|$. Then there is some $\tilde{\mu} \in\left[0, \mu_{\min }\right)$ such that

$$
\|y(\tilde{\mu})-d\|<\|y(\tilde{\mu})-w\| .
$$

Suppose $g(\tilde{\mu}) \notin[u, w)$. Then, by the continuity of $g$ there has to be some $\mu \in[0, \tilde{\mu}]$ such that $g(\mu)=w$. This contradicts the definition of $\mu_{\min }$. 
Consequently $g(\tilde{\mu}) \in[u, w)$. Hence, by SP, $\|y(\tilde{\mu})-g(\tilde{\mu})\|<\|y(\tilde{\mu})-w\|$. Since $w \in(g(\tilde{\mu}), d)$, by lemma 2.1 (iv), $\|y(\tilde{\mu})-g(\tilde{\mu})\|<\|y(\tilde{\mu})-d\|$. So by lemma 2.1 (iii), $\|y(\tilde{\mu})-w\|<\|y(\tilde{\mu})-d\|$. This contradicts (2.29).

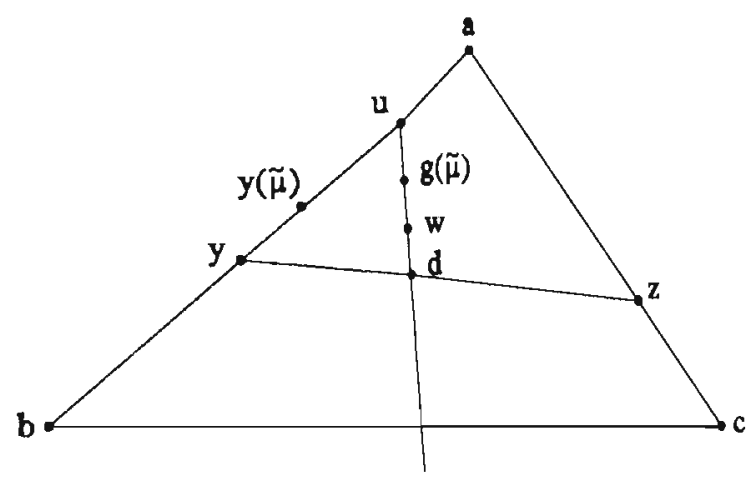

Figure 2.8: Proof of lemma 2.44

Figure 2.9 depicts the situation described in lemma 2.45: Here we have that $\varphi(a, b, c)=d$ and $w \in \Delta(a, b, c)$. Lemma 2.45 asserts that $\varphi(w, b, c)$ must also be on the line segment $[b, c \mid$ and that the line segments $[a, d]$ and $[w, \varphi(w, b, c)]$ must be parallel.

Lemma 2.45 Let assumption 2.3. Let $a, b, c, d, w, u \in \mathbb{R}^{2}$ be such that $d \in$ $(b, c), a, b, c$ noncollinear, $u \in[b, c], w \in \Delta(a, b, c) \backslash[b, c],(w, u) / /(a, d)$ and $\varphi(a, b, c)=d$. Then $\varphi(w, b, c)=u$.

Proof Since $\varphi(a, b, c)=d \notin\{a, b, c\}, \varphi$ is nondictatorial. Let $\hat{w}=$ $\varphi(w, b, c)$. (See figure 2.9.)

Suppose $\hat{w} \neq u$. Since $\varphi(a, b, c)=d \in(b, c)$, we have by lemma 2.40 that $b-c$ and $a-d$ are $\|\cdot\|$-orthogonal. So $b-c$ and $w-u$ are $\|\cdot\|$ orthogonal. Hence, certainly $w-u$ is $\|\cdot\|$-orthogonal to $b-c$ in the sense of Birkhoff. Since $\|\cdot\|$ is strictly convex, we have by lemma 2.33 that $\|w-u\|<\|w-(u+\lambda(b-c))\|$ for all $\lambda \in \mathbf{R} \backslash\{0\}$. So $\|w-u\|<\|w-x\|$ for all $x \in[b, c] \backslash\{u\}$. Since by lemma $2.34 \varphi(u, b, c)=u$, we have by SP that $\|w-\hat{w}\| \leq\|w-u\|$. So $\hat{w} \notin[b, c]$. Hence, theorem 2.1 implies that $\hat{w} \in \Delta(w, b, c) \backslash[b, c] \subset \Delta(a, b, c) \backslash[b, c]$.

Let $\hat{u} \in[b, c]$ be such that $(\hat{w}, \hat{u}) / /(a, d)$. W.l.o.g. assume $\hat{u} \in[b, d]$. Let $y=\varphi(\hat{w}, b, u)$. Because of lemmas 2.35 and 2.40 , we have $y \notin[\hat{w}, \hat{u})$. Furthermore by theorem 2.1, $y \in \Delta(\hat{w}, b, \hat{u})$. If $y \notin[\hat{w}, \hat{u}]$, then there are $\tilde{w} \in[y, \hat{w}, \rightarrow) \cap(d, a, \rightarrow)$ and $\tilde{u} \in[y, \hat{u}, \rightarrow) \cap[a, d, \rightarrow)$. In that case, by 


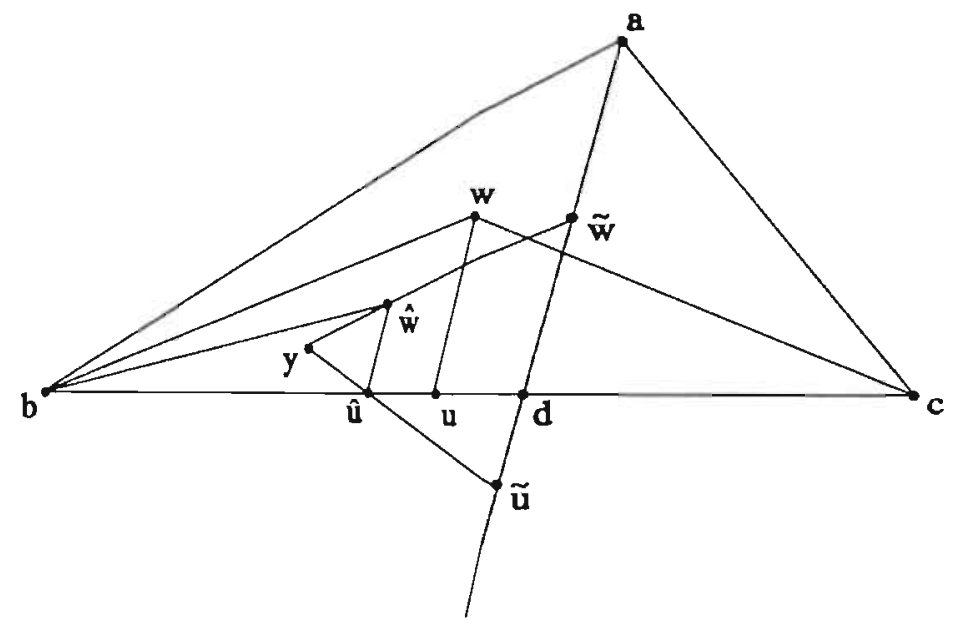

Figure 2.9: Proof of lemma 2.45

lemma $2.38, \varphi(\tilde{w}, b, u)=y$. But by lemma $2.38, \varphi(\tilde{w}, b, d)=d$. So by SP, $\|\tilde{u}-y\| \leq\|\tilde{u}-d\|$. Hence, $\|\tilde{u}-\hat{u}\|<\|\tilde{u}-d\|$. This contradicts lemma 2.40 .

Consequently, $y=\hat{u}$. By SP, $\|c-\hat{w}\| \leq\|c-\hat{u}\|$. Hence by lemma 2.1 (iii), $\left\|c-\left(\frac{1}{2} \hat{w}+\frac{1}{2} \hat{u}\right)\right\|<\|c-\hat{u}\|$, which contradicts lemma 2.40. Therefore $\hat{w}=u$.

Lemma 2.46 states that if a point $a$ domin ates two points $b$ and $c$, then every point inside the triangle $\Delta(a, b, c)$ dominates $b$ and $c$.

Note that this lemma excludes the situations which were studied in lemmas 2.43 and 2.44. These lemmas were only used to prove lemma 2.45 .

Lemma 2.46 Let assumption 2.9. Let $\varphi$ be nondictatorial. Let $a, b, c, d \in$ $\mathbf{R}^{2}$ such that $\varphi(a, b, c)=a$ and $d \in \Delta(a, b, c)$. Then $\varphi(d, b, c)=d$.

Proof In view of lemma 2.34 we assume that $a, b, c$ are noncollinear. Let $e=\varphi(d, b, c)$. Suppose $e \neq d$. W.l.o.g. $d \in[a, b]$. Then by lemma 2.44, there are $\tilde{c} \in(a, c, \rightarrow)$ and $\tilde{b} \in(d, b, \rightarrow)$ such that $\varphi(d, \tilde{b}, \tilde{c})=\tilde{e} \in(\tilde{b}, \tilde{c})$. (See figure 2.10.)

Let $x \in(\tilde{b}, \tilde{c})$ with $(d, x) / /(a, c)$. By lemma 2.35, we have $\|b-a\|<$ $\|b-z\|$ for all $z \in[\tilde{c}, a)$. Hence, $\|\tilde{b}-d\|<\| \tilde{b}-z_{i} \mid$ for all $z \in \Delta(x, d, \tilde{c}) \backslash\{d\}$. 


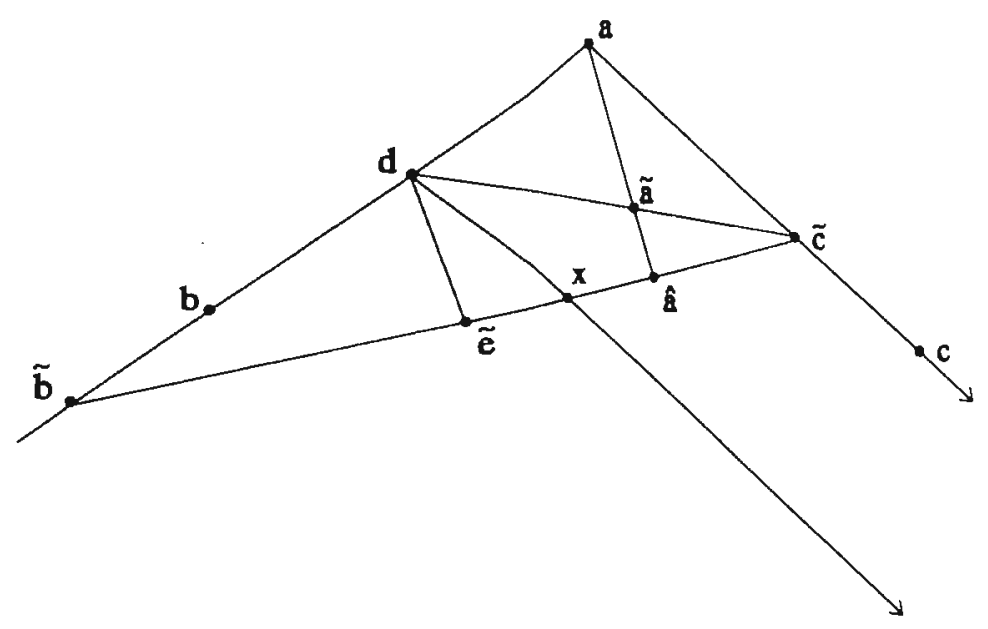

Figure 2.10: Proof of lemma 2. 46

Hence, $\tilde{e} \in[\tilde{b}, x)$. Consequently, there are $\tilde{a}$ and $\hat{a}$ such that $\tilde{a} \in(a, \hat{a})$ $\hat{a} \in[\tilde{c}, \tilde{b}),(a, \hat{a}) / /(d, \tilde{e})$ and $\tilde{a} \in(d, \tilde{c})$. Hence, $\varphi(\tilde{a}, \tilde{b}, \tilde{c})=\hat{a}$ by lemma 2.45 . So, $\varphi(a, \tilde{b}, \tilde{c})=\hat{a}$ by lemma 2.38. But $\varphi(a, \tilde{b}, \tilde{c})=a$ because of $\varphi(a, b, c)=a$ and lemma 2.38. So we have a contradiction. Consequently, $e=d$.

Next three lemmas are mainly concerned in establishing translation covariance. A voting scheme is translation covariant if in each case where all reported points are submitted to the same translation, the compromise point is also moved according to this translation.

Lemma 2.47 is only used to prove the more general lemma 2.48 .

Lemma 2.47 Let assumption 2.3. Let $a, b, c \in \mathbf{R}^{2}$ with $\varphi(a, b, c)=a$ and $a, b, c$ noncollinear. Let $\bar{a} \in(b, a), \bar{c} \in(b, c)$ such that $(\bar{a}, \bar{c}) / /(a, c)$. Then $\varphi(\bar{a}, b, \bar{c})=\bar{a}$.

Proof If $\varphi$ is dictatorial, $\varphi(a, b, c)=a$ implies that $\varphi(\bar{a}, b, \bar{c})=\bar{a}$. Now assume $\varphi$ is nondictatorial. Let $w=\varphi(\bar{a}, b, c)$. Suppose $w \notin[\bar{a}, \bar{c}]$. Then $[w, \bar{c}, \rightarrow) \cap[a, c, \rightarrow] \neq \emptyset$. Let $u \in[w, \bar{c}, \rightarrow) \cap[a, c, \rightarrow]$. Lemma 2.38 implies $\varphi(\bar{a}, b, u)=w$. On the other hand, by lemma $2.38, \varphi(a, b, u)=a$. Hence 
by lemma 2.46, $\varphi(\bar{a}, b, u)=\bar{a}$. So $w=\bar{a}$, a contradiction. Consequently, $w \in[\vec{a}, \bar{c}]$.

Now suppose $w \neq \bar{a}$. Hence by lemma 2.35, $\|b-w\|<\|b-\bar{a}\|$. Let $\tilde{w} \in[b, w, \rightarrow) \cap[c, a)$. Then:

$$
\|b-\tilde{w}\|<\|b-a\| .
$$

On the other hand by lemma 2.37, $\varphi(a, \tilde{w}, c)=\tilde{w}$, whereas $\varphi(a, b, c)=a$. Hence by SP, $\|b-a\| \leq\|b-\tilde{w}\|$, which contradicts (2.30).

Lemma 2.48 considers the situation depicted by figure 2.11: Here we have that $\varphi(a, b, c)=a, \bar{a}$ is on the half-line $[b, a, \rightarrow)$ and $\bar{c}$ is on the half-line $[b, c, \rightarrow)$. $\bar{a}$ and $\bar{c}$ are such that the line segment $[\bar{a}, \bar{c}]$ is parallel to $[a, c]$. Lemma 2.48 states that $\varphi(\bar{a}, b, \bar{c})=a$ (lemma 2.47 handled the subcase that $\bar{a}$ is between $a$ and $b$ ).

Lemma 2.48 Let assumption 2.9. Let $a, b, c \in \mathbf{R}^{2}$ with $\varphi(a, b, c)=a$ and $a, b, c$ noncollinear. Let $\bar{a} \in(b, a, \rightarrow)$ and $\bar{c} \in(b, c, \rightarrow)$ such that $(\bar{a}, \bar{c}) / /(a, c)$. Then $\varphi(\bar{a}, b, \bar{c})=\bar{a}$.

Proof If $\varphi$ is dictatorial, $\varphi(a, b, c)=a$ implies that $\varphi(\vec{a}, b, \bar{c})=\bar{a}$. Now assume $\varphi$ is nondictatorial. If $\bar{a} \in[b, a]$, then $\bar{c} \in[b, c]$ and thus $\varphi(\bar{a}, b, \bar{c})=\bar{a}$ because of lemma 2.47. Now assume $\bar{a} \notin[b, a]$. (See figure 2.11).

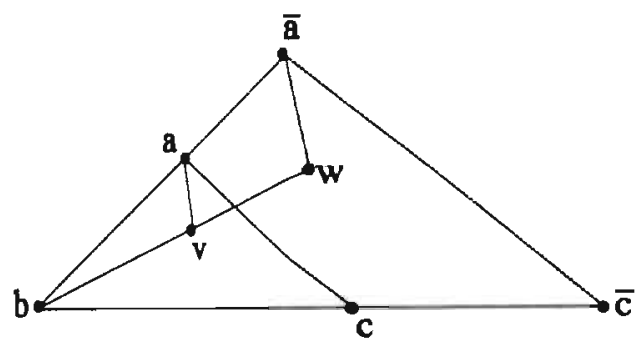

Figure 2.11: Proof of lemma 2.48

Let $w=\varphi(\bar{a}, b, \bar{c})$. By confirmatoriness (see lemma 1.4):

$$
w=\varphi(\bar{a}, b, w) .
$$

Suppose $w \notin[b, \bar{a}]$. Let $v \in[w, b]$ be such that $(a, v) / /(\bar{a}, w)$. (2.31) implies by lemma 2.47, $\varphi(a, b, v)=v$. On the other hand, $\varphi(a, b, c)=a$, so by SP, 
$\|c-a\| \leq\|c-v\|$. Hence, $\|\bar{c}-\bar{a}\| \leq\|\bar{c}-w\|$. This contradicts lemma 2.35 . Consequently, $w \in[b, \bar{a}]$. By $a=\varphi(a, b, c)$ and lemma 2.35, $\|c-a\|<\|c-x\|$ for all $x \in[b, a)$. So $\|\bar{c}-\bar{a}\|<\|\bar{c}-x\|$ for all $x \in[b, \bar{a})$. By lemma 2.34, $\varphi(\bar{u}, b, \bar{a})=\bar{a}$. By SP, $\|\bar{c}-w\| \leq\|\bar{c}-\bar{a}\|$, hence $w=\bar{a}$.

In lemma 2.49 translation covariance is established.

Lemma 2.49 Let assumption 2.3. Let $a, b, c, u \in \mathbb{R}^{2}$. Then $\varphi(a+u, b+$ $u, c+u)=\varphi(a, b, c)+u$.

Proof If $\varphi$ is dictatorial, the desired result is trivial. Now assume $\varphi$ is nondictatorial. If $a, b, c$ are collinear, the desired result follows from lemma 2.35. Now assume $a, b, c$ are noncollinear. Then by lemma 2.42 it is sufficient to prove: If $\varphi(a, b, c)=a$, then $\varphi(a+u, b+u, c+u)=a+u$. But this follows evidently from lemma 2.48 .

Lemma 2.50 considers the situation depicted by figure 2.12 where $\varphi(a, b, c)=$ $d$. The lemma asserts that $\varphi(a, u, c)=d$.

Lemma 2.50 Let assumption 2.3. Let $a, b, c \in \mathbb{R}^{2}$ be such that $\varphi(a, b, c)$ $=d \in(b, c)$. Let $u \in[a, d, \rightarrow) \backslash[a, d)$. Then $\varphi(a, u, c)=d$.

Proof Assume that $a \neq d$ (otherwise the desired result is trivial). Then since $\varphi(a, b, c) \notin\{a, b, c\}, \varphi$ is nondictatorial. By lemma 2.34, $a, b$ and $c$ are noncollinear. Let $a(\lambda)=d+\lambda(a-d)$ for $\lambda \in[0, \infty)$. (See figure 2.12.)

Let $w(\lambda)=\varphi(a(\lambda), u, c)$ for $\lambda \in[0, \infty)$. By lemma $2.38, \varphi(a(\lambda), b, c)=d$. Hence by SP,

$$
\|u-w(\lambda)\| \leq\|u-d\| \text { for all } \lambda \in[0, \infty)
$$

Lemma 2.40 implies that $a-d$ and $b-c$ are $\|\cdot\|$-orthogonal. Hence $a-d$ is $\|\cdot\|$-orthogonal to $b-c$ in the sense of Birkhoff. Since $\|\cdot\|$ is strictly convex, lemma 2.8 implies that $\|a-d\|<\| a-(d+\mu(b-c))||$ for all $\mu \in \mathbf{R} \backslash\{0\}$. Therefore,

$$
\|a-d\|<\|a-x\| \text { for all } x \in[c, d)
$$

and also

$$
\|u-d\|<\|u-x\| \text { for all } x \in[c, d) .
$$

By PO and theorem 2.1, $w(\lambda) \in \Delta(a(\lambda), u, c)$ for all $\lambda \in[0, \infty)$. Also by (2.32) and (2.34), $\|u-w(\lambda)\|<\|u-x\|$ for all $x \in[c, d)$ and all $\lambda \in[0, \infty)$. Thus, $w(\lambda) \in \Delta(d, u, c) \backslash[c, d)$ for all $\lambda \in[0, \infty) . \Delta(d, u, c)$ is a compact set. 


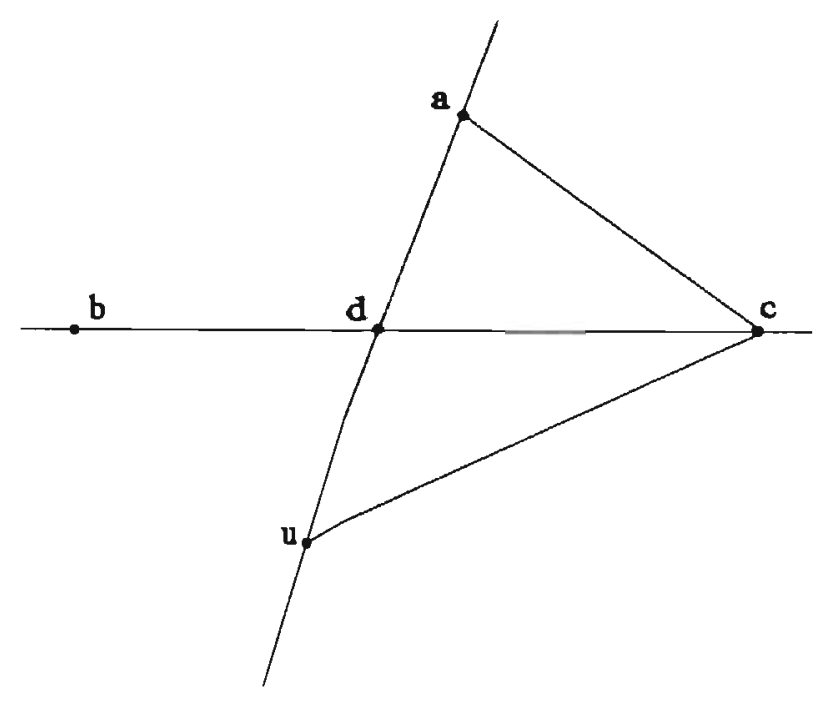

Figure 2.12: Proof of lemma 2.50

Now let $\lambda_{j} \in[0, \infty)$ be such that $\lambda_{j} \rightarrow \infty, \lambda_{1}=1$ and $w\left(\lambda_{j}\right) \rightarrow \bar{w}$ with $\bar{w} \in \Delta(d, u, c)$.

Suppose $w(1) \neq d$. Clearly, $\bar{w} \neq d$, otherwise (2.33) implies that $\| a-$ $w\left(\lambda_{j}\right)\|<\| a-w(1) \|$ for $\lambda_{j}$ large enough, contradicting SP. Suppose $\bar{w} \in$ $[c, d)$. In view of $(2.34)$ we can take $j \in \mathbb{N}$ such that $\left\|w\left(\lambda_{j}\right)-\bar{w}\right\|<$ $\|u-\bar{w}\|-\|u-d\|$. Hence, $\|u-d\|<\left\|u-w\left(\lambda_{j}\right)\right\|$. This contradicts (2.32). Consequently, $\bar{w} \notin[c, d)$. So $\bar{w} \in \Delta(u, d, c) \backslash[d, c]$. Let $y \in[c, d]$ with $(\bar{w}, y) / /(u, d)$. Let $z\left(\lambda_{j}\right) \in[d, c] \cap\left[w\left(\lambda_{j}\right), a\left(\lambda_{j}\right)\right]$. Hence, $z\left(\lambda_{j}\right) \rightarrow y$. So by lemma 2.35, $\left\|c-w\left(\lambda_{j}\right)\right\|<\left\|c-z\left(\lambda_{j}\right)\right\|$ for all $j \in \mathbb{N}$. Hence, $\|c-\bar{w}\| \leq\|c-y\|$. This contradicts the $\|\cdot\|$-orthogonality of $c-d$ and $a-d$.

Lemma 2.51 handles the same situation as lemma 2.50. (See figure 2.12.) It states that if the scheme $\varphi$ is also anonymous, it assigns $d$ to each profile which components are a subset of $\{a, b, c, u\}$ with cardinality 3 .

Lemma 2.51 Let assumption 2.9. Let $\varphi$ satisfy $A N$. Let $a, b, c, d, u \in \mathbf{R}^{2}$ be such that $d \in(b, c)$ and $d \in(a, u)$ while $a, b, c$ are noncollinear. Let $x, y, z \in\{a, b, c, u\}$ be such that $|\{x, y, z\}|=3$. Then $\varphi(a, b, c)=d$ if and only if $\varphi(x, y, z)=d$.

Proof Since $\varphi(a, b, c)=d$, we have by AN that $\varphi(a, c, b)=d$. The desired result now can be obtained by repeated application of lemma 2.50 . 
The following lemma is only used in the proof of lemma 2.53 .

Lemma 2.52 Let assumption 2.3. Let $a, b, c, d, x \in \mathbf{R}^{2}$ with $b, c \in[a, d]$, $\varphi(a, c, x)=x, \varphi(b, d, x)=x$. Then $\varphi(b, c, x)=x$.

Proof Let $z=\varphi(b, c, x)$. By $\varphi(a, c, x)=x$ and SP:

$$
\|a-x\| \leq\|a-z\| .
$$

By $\varphi(b, d, x)=x$ and SP:

$$
\|d-x\| \leq\|d-z\|
$$

By theorem 2.1, $z \in \Delta(b, c, x)$. If $x \in[a, d]$, this implies that $z \in[a, d]$ and hence by (2.35) and (2.36), $z=x$. Now assume that

$$
x \notin[a, d] .
$$

Suppose $z \neq x$. Then we can take $u \in[b, c]$ with $z \in[u, x)$. By lemma 2.1 (iv), (2.35) implies that $\|a-x\| \leq\|a-u\|$. Similarly by (2.36), $\|d-x\| \leq\|d-u\|$. Hence, $\|a-x\|+\|x-d\|=\|a-d\|$. So by lemma 2.1 (v), $x \in[a, d]$. This contradicts (2.37). Consequently, $z=x$.

In lemmas 2.50 and 2.51 we assumed that there are $a, b, c, d \in \mathbb{R}^{2}$ with $\varphi(a, b, c)=d \neq a$ and $d \in(b, c)$. Lemma 2.53 guarantees the existence of such $a, b, c, d$, enabling us to apply lemmas 2.50 and 2.51 .

Lemma 2.53 Let assumption 2.3. Let $\varphi$ satisfy AN. Then there are noncollinear $x, y, z \in \mathbf{R}^{2}$ such that $\varphi(x, y, z) \in(y, z)$.

Proof By AN, $\varphi$ is nondictatorial. Take $\tilde{a}, b, c \in \mathbf{R}^{2}$ such that $\tilde{a}, b, c$ are noncollinear and $\|\tilde{a}-\boldsymbol{b}\|=\|\tilde{a}-c\|$. Let $a=\varphi(\tilde{a}, b, c)$. If $a=b$, lemma 2.35 implies that $\|\tilde{a}-b\|=\|\tilde{a}-a\|<\|\tilde{a}-c\|$, contradicting our assumption. So $a \neq b$. Similarly, $a=c$. If $a \in(b, c)$, we are done. Now assume

$$
a \notin(b, c) \text {. }
$$

If $a=b$, lemma 2.37 implies that $\|\tilde{a}-b\|=\|\tilde{a}-a\|<\|\tilde{a}-c\|$, contradicting our assumption. So $a \neq b$. Similarly, $a \neq c$. Hence, by $(2.38), a \notin[b, c]$. By lemma 2.34, $a, b$ and $c$ are noncollinear. Confirmatoriness (see lemma 1.4) implies $\varphi(a, b, c)=a$. Let $u(\lambda)=a+\lambda(a-c), \lambda \in[0, \infty)$. For $\lambda$ large, 
$\|b-c\|<\|b-u(\lambda)\|$, and therefore by SP and lemma 2.33, $\varphi(u(\lambda), b, c) \neq$ $u(\lambda)$.

Let $\lambda_{\max }=\sup \{\lambda \in[0, \infty]: \varphi(d(\lambda), b, c)=d(\lambda)\}$. Then $\lambda_{\max }<\infty$. Let $d=d\left(\lambda_{\max }\right)$. Then by continuity (see lemma 2.36 ):

$$
\varphi(d, b, c)=d
$$

By definition of $d$ :

$$
\varphi(x, b, c) \neq x \text { for all } x \in[c, d, \rightarrow) \backslash[c, d] .
$$

Let $v \in[c, d, \rightarrow \backslash \backslash[c, d]$ be such that:

$$
\|v-d\|<\|v-b\| .
$$

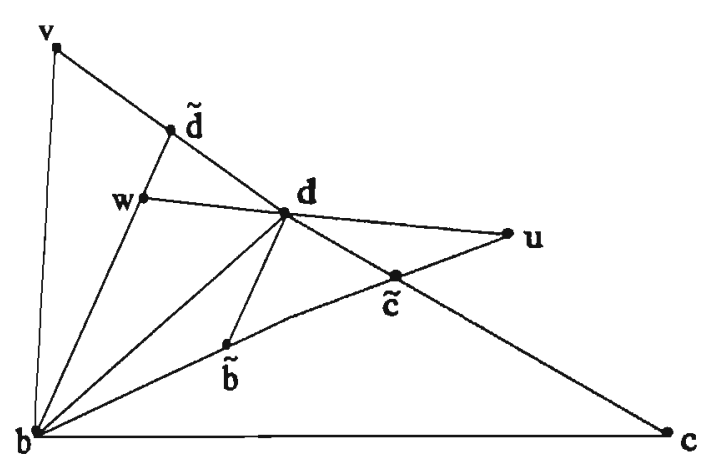

Figure 2.13: Proof of lemma 2.59

Let $w=\varphi(v, b, d)$. Suppose $w \notin[b, d]$. (See figure 2.13.) Then by PO and theorem 2.1, $w \in \Delta(v, b, d) \backslash[b, d]$. Confirmatoriness implies $\varphi(w, b, d)=w$. Choose $u \in[w, d, \rightarrow) \backslash w, d]$ arbitrarily. By lemma 2.38, $\varphi(w, b, u)=w$. Let $\tilde{b} \in[b, u]$ with $(\tilde{b}, d) / /(b, w)$. Let $\tilde{c} \in[d, c, \rightarrow) \cap[b, u]$. Then by lemma 2.48, $\varphi(d, \tilde{b}, u)=d$. On the other hand, (2.39) and lemma 2.38 imply $\varphi(d, b, \tilde{c})=$ $d$. So by lemma $2.52, \varphi(d, \tilde{b}, \tilde{c})=d$. Let $\tilde{d} \in[b, w, \rightarrow] \cap[v, d)$. Then by lemma $2.48, \varphi(\tilde{d}, b, \tilde{c})=\tilde{d}$. So by lemma $2.38, \varphi(\tilde{d}, b, c)=\tilde{d}$. This contradicts (2.40). Consequently, $w \in[b, d]$. By (2.41) and lemma 2.35, $w \neq b$, hence $w \in[d, b)$. If $w \neq d$, then $\varphi(v, b, d)=w \in(b, d)$, and we are done. Now assume $w=d$. By lemma 2.34, $\varphi(v, d, c)=d$. Hence, lemma 2.42 implies $\varphi(v, b, c)=d \in(v, c)$. So by lemma 2.51, $\varphi(b, v, c)=d \in(v, c)$. 
Lemma 2.54 Let assumption 2.9. Let $\varphi$ satisfy AN. Let noncollinear $a, b, c$ $\in \mathbf{R}^{2}$ be such that $\varphi(a, b, c)=d \in(b, c)$. Let $y \in(\leftarrow, a, d, \rightarrow), z \in(\leftarrow, b, c, \rightarrow)$. Then $\varphi(d, y, z)=d$.

Proof Let $u=2 d-a$. W.1.o.g. $y \in[d, u, \rightarrow), z \in[d, b, \rightarrow]$. Lemma 2.51 implies $\varphi(a, u, b)=d$. So by lemma $2.38, \varphi(d, y, z)=d$.

We are now in a position to derive the main result of this section. In the next section this result will be generalized for an arbitrary number of agents.

Theorem 2.4 Let $\|\cdot\|$ be a strictly convex norm. Let $\varphi:\left(\mathbb{R}^{2}\right)^{3} \rightarrow \mathbb{R}^{2}$ satisfy anonymity, and Pareto optimality and strategy-proofness with respect to $\|\cdot\|$. Then $\varphi$ is the coordinatewise median scheme with respect to some $\|\cdot\|$-orthogonal pair of nonzero vectors without constant points.

Proof By AN, $\varphi$ is nondictatorial. By lemma 2.53 there are noncollinear $a, b, c, d \in \mathbf{R}^{2}$ such that $\varphi(a, b, c)=d \in(b, c)$. Let $u=c-d, v=a-d$. Then $u$ and $v$ are linearly independent and by lemma 2.40 they are $\|\cdot\|$-orthogonal. Let $\psi$ be the coordinatewise median scheme with respect to $\{v, w\}$ without constant points.

Suppose $\varphi \neq \psi$. Then we can take $x, y, z \in \mathbf{R}^{2}$ such that $\varphi(x, y, z) \neq$ $\psi(x, y, z)$. Lemma 2.20 implies that $\psi$ is Pareto optimal with respect to $\|\cdot\|$. In view of proposition 2.1 and the anonymity of $\psi$ we may assume that $\|\psi(x, y, z)-x\|<\|\varphi(x, y, z)-x\|$. Let $r=\psi(x, y, z)$. Then it is trivial that $\psi(r, y, z)=r$. Furthermore by SP, $\|\varphi(x, y, z)-x\| \leq\|\varphi(r, y, z)-x\|$. Hence, $\varphi(r, y, z) \neq r$. In view of lemma 2.49 we may assume that $r=d$. By the definition of $\psi,\left|\left\{\tilde{x} \in\{d, y, z\}: x_{j}=d_{j}\right\}\right| \geq 2$ for $j=1,2$.

Also by lemma 2.33, $y \neq d$ and $z \neq d$. So $y, z \in(\leftarrow, d, d+u, \rightarrow) \cup$ $(\leftarrow, d, d+v, \rightarrow)$. If $d, y$ and $z$ are noncollinear, lemma 2.54 implies that $\varphi(d, y, z)=d$, a contradiction. So $d, y$ and $z$ are collinear. Hence by the definition of $\psi, d \in[y, z]$. So by lemma 2.34, $\varphi(d, y, z)=d$, the same contradiction. Consequently, $\varphi=\psi$.

\section{7 $n$-person location problems in $\mathbb{R}^{2}$}

In the preceding section a complete characterization is obtained of all schemes of the 3-person location problem in $\mathbf{R}^{2}$, which satisfy $\mathrm{AN}$ and PO and SP with respect to a strictly convex norm $\|\cdot\|$. In this section we generalize this result for an arbitrary number of agents. 
Lemma 2.55 Let $\varphi:\left(\mathbb{R}^{2}\right)^{N} \rightarrow \mathbb{R}^{2}$ satisfy $A N$, and $S P$ and $P O$ with respect to a strictly convex norm $\|\cdot\|$. Then there is a coordinatewise median scheme $\psi:\left(\mathbb{R}^{2}\right)^{3} \rightarrow \mathbb{R}^{2}$ with respect to some $\|\cdot\|$-orthogonal $u, v \in \mathbb{R}^{2} \backslash\{0\}$ without constant points such that:

(i) For all disjoint $J, K, L \subset N$ with $J \cup K \cup L=N$ and $|J|,|K|,|L|<\frac{1}{2} n$ we have that $\varphi\left(\left\langle x^{J}, y^{K}, z^{L}\right\rangle\right) \equiv \psi(x, y, z)$.

(ii) For all disjoint $J, K, L$ with $J \cup K \cup L=N$ we have for all $x, y, z \in \mathbb{R}^{2}$ :

$$
\begin{aligned}
\varphi\left(\left\langle x^{J}, y^{K}, z^{L}\right\rangle\right) & =x \text { if }|J|>\frac{1}{2} n \\
& =y \text { if }|K|>\frac{1}{2} n \\
& =z \text { if }|L|>\frac{1}{2} n .
\end{aligned}
$$

Proof Corollary 2.6 implies that $n$ is odd. Take arbitrary disjoint $J, K, L \subset$ $N$ with $J \cup K \cup L=N$. Suppose $|J|>\frac{1}{2} n$ and $\varphi\left(\left\langle x^{J}, y^{K}, z^{L}\right\rangle\right) \neq x$ for some $x, y, z \in \mathbf{R}^{2}$. Let $w=\varphi\left(\left\langle x^{J}, y^{K}, z^{L}\right\rangle\right)$. Then by confirmatoriness (see lemma 1.4), $\varphi\left(\left\langle x^{J}, w^{K \cup L}\right\rangle\right)=w \neq x$. This contradicts lemma 2.32 . Consequently, $\varphi\left(\left\langle x^{J}, y^{K}, z^{L}\right\rangle\right)=x$ if $|J|>\frac{1}{2} n$. Similarly we can prove that $\varphi\left(\left\langle x^{J}, y^{K}, z^{L}\right\rangle\right)=y$ if $|K|>\frac{1}{2} n$ and $\varphi\left(\left\langle x^{J}, y^{K}, z^{L}\right\rangle\right)=z$ if $|L|>\frac{1}{2} n$. By this (ii) is proved.

Now assume $|J|,|K|,|L|<\frac{1}{2} n$. Let disjoint $R, S, T \subset N$ with $|R|=1$ and $|S|=|T|=\frac{n-1}{2}$. Let $\psi:\left(\mathbf{R}^{2}\right)^{3} \rightarrow \mathbf{R}^{2}$ be defined by $\psi(x, y, z)=$ $\varphi\left(\left\langle x^{R}, y^{S}, z^{T}\right\rangle\right)$. Then by lemma 2.41, $\psi$ satisfies $\mathrm{AN}$, and PO and SP with respect to $\|\cdot\|$. So, by theorem $2.4, \psi$ is the coordinatewise median scheme with respect to some $\|\cdot\|$-orthogonal pair of vectors $u, v \in \mathbb{R}^{2} \backslash\{0\}$ without constant points. Similarly we can define the coordinatewise median scheme $\tilde{\psi}:\left(\mathbb{R}^{2}\right)^{3} \rightarrow \mathbb{R}^{2}$ with respect to some $\|\cdot\|$-orthogonal pair of vectors $\tilde{u}, \tilde{v} \in$ $\mathrm{R}^{2} \backslash\{0\}$ without constant points such that $\varphi\left(\left\langle x^{J}, y^{K}, z^{L}\right\rangle\right) \equiv \tilde{\psi}(x, y, z)$. Suppose $\psi \neq \tilde{\psi}$. Then there are $x, y, z \in \mathbf{R}^{2}$ with $\psi(x, y, z) \neq \tilde{\psi}(x, y, z)$. In view of the anonymity of $\psi$ and $\tilde{\psi}$ and proposition 2.1 we assume w.l.o.g. that

$$
\|\psi(x, y, z)-x\|<\|\tilde{\psi}(x, y, z)-x\| .
$$

On the other hand, let disjoint $R^{\prime}, S^{\prime}, T^{\prime} \subset N$ be such that $\left|R^{\prime}\right|=1$, $\left|S^{\prime}\right|=\left|T^{\prime}\right|=\frac{n-1}{2}, R^{\prime} \subset J, K \subset S^{\prime}$ and $L \subset T^{\prime}$. Lemma 1.1 implies that $\varphi$ satisfies ISP, so $\left\|\varphi\left(\left\langle x^{J}, y^{K}, z^{L}\right\rangle\right)-x\right\| \leq\left\|\varphi\left(\left\langle x^{R^{\prime}}, y^{S^{\prime}}, z^{T^{\prime}}\right\rangle\right)-x\right\|$. Hence by AN, $\left\|\varphi\left(\left\langle x^{J}, y^{K}, z^{L}\right\rangle\right)-x\right\| \leq\left\|\varphi\left(\left\langle x^{R}, y^{S}, z^{T}\right\rangle\right)-x\right\|$. Therefore, $\|\tilde{\psi}(x, y, z)-x\| \leq\|\psi(x, y, z)-x\|$. This contradicts (2.42). Consequently, $\psi=\tilde{\psi}$. This proves (i). 
We are now sufficiently equipped to derive one of the main results of this chapter. The following theorem is a generalization of theorem 2.4 for an arbitrary number of agents.

Theorem 2.5 Let $\|\cdot\|$ be a strictly convex norm. Let the number of agents $n$ be odd. Let $\varphi:\left(\mathbf{R}^{2}\right)^{N} \rightarrow \mathbb{R}^{2}$ be a voting scheme. Then $\varphi$ satisfies anonymity, and (weak) Pareto optimality and strategy-proofness with respect to $\|\cdot\|$ if and only if $\varphi$ is the coordinatewise median scheme with respect to some linearly independent $\|\cdot\|$-orthogonal pair of vectors without constant points.

Proof By proposition 2.1 PO and WPO with respect to $\|\cdot\|$ are equivalent. The if-part of the proof follows by lemmas 2.19 and 2.20 and the observation that all coordinatewise median schemes satisfy anonymity. Now let $\varphi$ : $\left(\mathbf{R}^{2}\right)^{N} \rightarrow \mathbf{R}^{2}$ satisfy AN, and PO and SP with respect to $\|\cdot\|$. Let $A(j)=$ $\left\{p \in\left(\mathbb{R}^{2}\right)^{N}:|\{p(i): i \in N\}| \leq j\right\}$. By lemma 2.55 we can take $\|\cdot\|-$ orthogonal $u, v \in \mathbb{R}^{2} \backslash\{0\}$ such that $\varphi(p)=\psi(p)$ for all $p \in A(3)$, where $\psi:\left(\mathbf{R}^{2}\right)^{N} \rightarrow \mathbf{R}^{2}$ is the coordinatewise median scheme with respect to $\{u, v\}$ without constant points. Now assume

$$
\varphi(p)=\psi(p) \text { for all } p \in A(j),
$$

with $j \in\{3, \ldots, n-1\}$. We will prove that $\varphi(p)=\psi(p)$ for all $p \in A(j+1)$; the desired result then follows by induction (note that $\left(\mathbb{R}^{2}\right)^{N}=A(n)$ ).

Let $p \in A(j+1)$ be arbitrary. Let $w=\varphi(p)$. Let $\lambda, \mu: \mathbf{R}^{2} \rightarrow \mathbf{R}$ be defined by $x=\lambda(x) u+\mu(x) v$ for all $x \in \mathbf{R}^{2}$. So $\psi(q)=\operatorname{med}(\lambda(q(1)), \ldots, \lambda(q(n))) u+$ $\operatorname{med}(\mu(q(1)), \ldots, \mu(q(n))) v$ for all $q \in\left(\mathbb{R}^{2}\right)^{N}$. Let $\alpha=\lambda(w), \beta=\mu(w)$. Suppose $\alpha<\operatorname{med}(\lambda(p(1)), \ldots, \lambda(p(n)))$.

Let $\tilde{\alpha} \in \mathbf{R}$ be such that $\alpha<\tilde{\alpha} \leq \min \{\lambda(p(i)): \lambda(p(i))>\alpha\}$. Let $q \in A(j+1)$ be such that $q(i)=w$ if $\lambda(p(i)) \leq \alpha$, while $\lambda(q(i))=\tilde{\alpha}$ and $q(i) \in[w, p(i)]$ if $\lambda(p(i))>\alpha$. Positive association (lemma 2.13) implies $\varphi(q)=w$. Let $r \in A(j+1)$ be such that $r(i) \in\{q(i), w\}$, while $\mid\{i \in N$ : $r(i)=w\} \mid=\frac{n-1}{2}$. Then by confirmatoriness (see lemma 1.4), $\varphi(r)=w$. Hence, $\lambda(\varphi(r))=\alpha<\tilde{\alpha}=\operatorname{med}(\lambda(r(1)), \ldots, \lambda(r(n)))$. Because of $(2.43)$, $r \notin A(j)$. Hence, $\mid\{r(i): i \in N, \lambda((r(i))=\tilde{\alpha}\} \mid \geq 3$. W.l.o.g. $\mid\{r(i): i \in N$, $\lambda(r(i))=\tilde{\alpha}, \mu(r(i)) \geq \beta\} \mid \geq 2$. Let $k, l \in N$ be such that $\lambda(r(k))=\lambda(r(l))=$ $\tilde{\alpha}$ and $\mu(r(l))>\mu(r(k)) \geq \beta$. Let $s \in\left(\mathbf{R}^{2}\right)^{N}$ be such that $s(i)=r(k)$ if $r(i)=r(l)$ and $s(i)=r(i)$ if $r(i) \neq r(l)$. (See figure 2.14.)

So $s \in A(j)$. Hence by (2.43), $\varphi(s)=\tilde{\alpha} u+\eta v$, with $\eta \in[\beta, \mu(r(k))]$. SP implies:

$$
\|r(l)-w\| \leq\|r(l)-\varphi(s)\|
$$




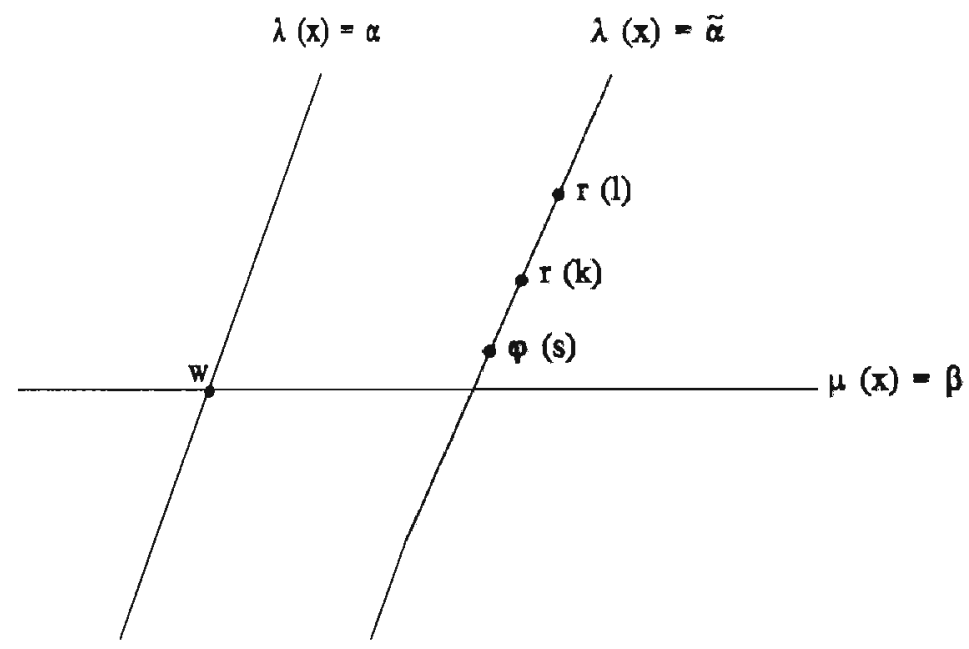

Figure 2.14: Proof of theorem 2.5

On the other hand, $u$ and $v$ are $\|\cdot\|$-orthogonal, hence in view of lemma 2.8,

$$
\|r(l)-w\|>\|r(l)-\varphi(s)\| .
$$

This contradicts (2.44). Consequently, $\alpha \geq \operatorname{med}(\lambda(p(1)), \ldots, \lambda(p(n)))$. Similarly, one can prove $\alpha \leq \operatorname{med}(\lambda(p(1)), \ldots, \lambda(p(n)))$. Hence, $\alpha=\operatorname{med}(\lambda(p(1))$, $\ldots, \lambda(p(n)))$. Analogously, $\beta=\operatorname{med}(\mu(p(1)), \ldots, \mu(p(n)))$. Since $p \in A(j+$ 1) was chosen arbitrarily, this means that $\varphi(p)=\psi(p)$ for all $p \in A(j+1)$.

The following theorem guarantees the existence of schemes under the conditions described in theorem 2.5 .

Theorem 2.6 Let $\|\cdot\|$ be a strictly convex norm. Let the number of agents $n$ be odd. Then there exists a voting scheme $\varphi:\left(\mathbf{R}^{2}\right)^{N} \rightarrow \mathbf{R}^{2}$ which satisfies anonymity, and (weak) Pareto optimality and strategy-proofness with respect to $\|\cdot\|$.

Proof By corollary 2.3 we can take nonzero $u, v \in \mathbb{R}^{2}$ such that $u$ and $v$ are $\|\cdot\|$-orthogonal. Lemma 2.4 implies that $u$ and $v$ are linearly independent. Let $\varphi$ be the coordinatewise median scheme with respect to $\{u, v\}$ without constant points. Then the if-part of theorem 2.5 implies that $\varphi$ satisfies anonymity, and (weak) Pareto optimality and strategy-proofness with 
respect to $\|\cdot\|$.

For an even number of agents there are no schemes satisfying anonymity, and Pareto optimality and strategy-proofness with respect to some strictly convex norm $\|\cdot\|$, as is stated by theorem 2.3. In contrast to theorems 2.5 and 2.6, this theorem is also true for all nonempty subsets $A$ of $\mathbf{R}^{m}$, with $m \in\{2,3,4, \ldots\}$.

For an odd number of agents and $m \geq 3$ there are strictly convex norms on $\mathbf{R}^{m}$ for which no anonymous, Pareto optimal strategy-proof (with respect to this norm) scheme exists.

Theorem 3.2 states that with respect to norms which are induced by an inner product no such solutions exist. An example of such a norm is the Euclidean norm (see chapter 3 for further discussion).

On the other hand, there are also strictly convex norms for which a coordinatewise median scheme can be defined which satisfies AN, and SP and PO with respect to this norm, as the following example shows.

Example 2.13 Let $N=\{1,2,3\}$. Let $\|\cdot\|: \mathbf{R}^{3} \rightarrow \mathbf{R}$ be defined by $\|x\|=$ $\|x\|_{1}+\varepsilon\|x\|_{2}$, where $\varepsilon \in\left(0, \frac{1}{3}\right),\|\cdot\|_{1}$ denotes the sumnorm on $\mathbf{R}^{3}$ (i.e. $\|x\|_{1}=\sum_{j=1}^{3}\left|x_{j}\right|$ for all $\left.x \in \mathbb{R}^{3}\right)$ and $\|x\|_{2}$ denotes the Euclidean norm on $\mathbf{R}^{3}$. Let $\varphi:\left(\mathbf{R}^{3}\right)^{N} \rightarrow \mathbf{R}^{3}$ be the coordinatewise median scheme with respect to the standard basis without constant points. Then $\|\cdot\|$ is a strictly convex norm, and $\varphi$ satisfies $\mathrm{AN}$, and $\mathrm{PO}$ and SP with respect to $\|\cdot\|$.

Proof It is easy to verify that $\|\cdot\|$ is indeed a norm. Furthermore, since $\|\cdot\|_{2}$ is strictly convex, we have by lemma 2.1 (v) that for all $x, y, z \in \mathbb{R}^{3}$ such that $x \notin[y, z]$ :

$$
\begin{aligned}
& \quad\|y-z\|=\|y-z\|_{1}+\varepsilon\|y-z\|_{2}<\|x-y\|_{1}+ \\
& \quad\|x-z\|_{1}+\varepsilon\|x-y\|_{2}+\varepsilon\|x-z\|_{2}=\|x-y\|+\|x-z\| .
\end{aligned}
$$

(Here we use the fact that the Euclidean norm is strictly convex, cf. section 3.2.) Consequently, lemma $2.1(\mathrm{v})$ implies that $\|\cdot\|$ is a strictly convex norm. The anonymity of $\varphi$ is evident. Since the standard basis of $\mathbf{R}^{3}$ is $\|\cdot\|$-orthogonal, lemma 2.19 implies that $\varphi$ satisfies $\mathrm{SP}$ with respect to $\|\cdot\|$. So it remains to prove that $\varphi$ satisfies PO with respect to $\|\cdot\|$.

Suppose $\varphi$ is not Pareto optimal with respect to $\|\cdot\|$. Then there are $a, b, c, y, w \in \mathbf{R}^{3}$ with $w=\varphi(a, b, c)$,

$$
y \neq w,
$$


$\|y-a\| \leq\|w-a\|,\|y-b\| \leq\|w-b\|$ and $\|y-c\| \leq\|w-c\|$. W.l.o.g. we assume that

$$
w=0
$$

Then by lemma 2.1 (iii):

$$
\|a-\lambda y\|<\|a\|,\|b-\lambda y\|<\|b\| \text { and }\|c-\lambda y\|<\|c\| \text { for all } \lambda \in(0,1) \text {. }
$$

Hence, $a, b$ and $c$ are nonzero. So

$$
\begin{aligned}
\|a-\lambda y\|_{2} & =\sqrt{\left(\lambda y_{1}-a_{1}\right)^{2}+\left(\lambda y_{2}-a_{2}\right)^{2}+\left(\lambda y_{3}-a_{3}\right)^{2}} \\
& =\sqrt{a_{1}^{2}+a_{2}^{2}+a_{3}^{2}-2\left(a_{1} y_{1}+a_{2} y_{2}+a_{3} y_{3}\right) \lambda+o(\lambda)} \\
& =\sqrt{a_{1}^{2}+a_{2}^{2}+a_{3}^{2}} \sqrt{1-2 \frac{\left(a_{1} y_{1}+a_{2} y_{2}+a_{3} y_{3}\right)}{a_{1}^{2}+a_{2}^{2}+a_{3}^{2}} \lambda+o(\lambda)} \\
& =\sqrt{a_{1}^{2}+a_{2}^{2}+a_{3}^{2}}\left(1-\frac{\left(a_{1} y_{1}+a_{2} y_{2}+a_{3} y_{3}\right)}{a_{1}^{2}+a_{2}^{2}+a_{3}^{2}} \lambda+o(\lambda)\right) \\
& =\|a\|_{2}-\frac{\left(a_{1} y_{1}+a_{2} y_{2}+a_{3} y_{3}\right)}{\sqrt{a_{1}^{2}+a_{2}^{2}+a_{3}^{2}}} \lambda+o(\lambda) \text { for all } \lambda \in(0,1) .
\end{aligned}
$$

Here, $o(\lambda)$ denotes an arbitrary function of $\lambda$ such that

$$
\lim _{\lambda \downarrow 0} \frac{o(\lambda)}{\lambda}=0
$$

Furthermore, $\| a-\left.\lambda y\right|_{1}=\left|a_{1}-\lambda y_{1}\right|+\left|a_{2}-\lambda y_{2}\right|+\left|a_{3}-\lambda y_{3}\right|$ with $\left|a_{j}-\lambda y_{j}\right|=$ $\left|a_{j}\right|-f\left(a_{j}, y_{j}\right) \cdot \lambda+o(\lambda)$, where $f: \mathbb{R} \times \mathbb{R} \rightarrow \mathbb{R}$ is defined by $f(\mu, \gamma)=-\boldsymbol{\gamma}$ if $\mu<0, f(\mu, \gamma)=-|\gamma|$ if $\mu=0$, and $f(\mu, \gamma)=\gamma$ if $\mu>0$. Substitution in (2.47) yields:

$$
\begin{aligned}
& \left|a_{1}\right|+\left|a_{2}\right|+\left|a_{3}\right|-\left(f\left(a_{1}, y_{1}\right)+f\left(a_{2}, y_{2}\right)+f\left(a_{3}, y_{3}\right)\right) \lambda+\varepsilon\|a\|_{2}- \\
& \frac{\varepsilon \hat{a}_{1} y_{1}+a_{2} y_{2}+a_{3} \psi_{3}}{\sqrt{a_{1}^{2}+a_{2}^{2}+a_{3}^{2}}} \lambda+o(\lambda)<\|a\|_{1}+\varepsilon\|a\|_{2} \text { for all } \lambda \in(0,1) .
\end{aligned}
$$

Hence, by taking the limit of $\lambda$ to 0 , we obtain that:

$$
f\left(a_{1}, y_{1}\right)+f\left(a_{2}, y_{2}\right)+f\left(a_{3}, y_{3}\right)+\varepsilon \frac{a_{1} y_{1}+a_{2} y_{2}+a_{3} y_{3}}{\sqrt{a_{1}^{2}+a_{2}^{2}+a_{3}^{2}}} \geq 0
$$

So,

$$
f\left(a_{1}, y_{1}\right)+f\left(a_{2}, y_{2}\right)+f\left(a_{3}, y_{3}\right)+\varepsilon\left|y_{1}\right|+\varepsilon\left|y_{2}\right|+\varepsilon\left|y_{3}\right| \geq 0 .
$$

Similarly we find:

$$
f\left(b_{1}, y_{1}\right)+f\left(b_{2}, y_{2}\right)+f\left(b_{3}, y_{3}\right)+\varepsilon\left|y_{1}\right|+\varepsilon\left|y_{2}\right|+\varepsilon\left|y_{3}\right| \geq 0
$$


and:

$$
f\left(c_{1}, y_{1}\right)+f\left(c_{2}, y_{2}\right)+f\left(c_{3}, y_{3}\right)+\varepsilon\left|y_{1}\right|+\varepsilon\left|y_{2}\right|+\varepsilon\left|y_{3}\right| \geq 0 .
$$

By the definition of $\varphi, 0=\operatorname{med}\left(a_{j}, b_{j}, c_{j}\right)$ for $j=1,2,3$. From the definition of $f$ it is now easy to verify that

$$
f\left(a_{j}, y_{j}\right)+f\left(b_{j}, y_{j}\right)+f\left(c_{j}, y_{j}\right) \leq-\left|y_{j}\right| \text { for } j=1,2,3 .
$$

Using this result we obtain by summation of (2.48), (2.49) and (2.50):

$$
-\left|y_{1}\right|-\left|y_{2}\right|-\left|y_{3}\right|+3 \varepsilon\left|y_{1}\right|+3 \varepsilon\left|y_{2}\right|+3 \varepsilon\left|y_{3}\right| \geq 0 .
$$

Since $\varepsilon \in\left(0, \frac{1}{3}\right)$, this implies that $\left|y_{1}\right|+\left|y_{2}\right|+\left|y_{3}\right|=0$. So $y=0$, which leads together with (2.45) and (2.46) to a contradiction. Consequently, $\varphi$ is Pareto optimal with respect to $\|\cdot\|$.

\subsection{Coalitional strategy-proofness}

In this section we consider schemes $\varphi: A^{N} \rightarrow A$, where $A$ is an open subset of $\mathbb{R}^{m}, m \geq 2$. We show that if $\varphi$ satisfies Pareto optimality and coalitional strategy-proofness with respect to a strictly convex norm, $\varphi$ must be dictatorial. Next we show that Pareto optimality can be replaced by surjectivity or unanimity, to obtain the same result.

If we compare these results to theorem 2.5 , we see that for $m \geq 2, n \geq 2$ and $n$ even, CSP is far more restrictive thatn SP with respect to a strictly convex norm.

Lemma 2.56 Let $A$ be an open subset of $\mathbf{R}^{m}, m \geq 2$. Let $\varphi: A^{N} \rightarrow A$ satisfy $P O$ and CSP with respect to a strictly convex norm $\|\cdot\|$. Then $\varphi(p) \in\{p(i): i \in N\}$ for all profiles $p \in A^{N}$.

Proof Let $p \in A^{N}$. Suppose $\varphi(p) \notin\{p(i): i \in N\}$. Then there exists $\hat{p} \in A^{N}$ such that $\varphi(\hat{p}) \notin\{\hat{p}(i): i \in N\}$ and $|\{\hat{p}(i): i \in N\}|$ is minimal. By the openness of $A$ there is an $\varepsilon \in\left(0, \min _{i \in N}\|\hat{p}(i)-\varphi(\hat{p})\|\right)$ such that $B=\left\{x \in \mathbf{R}^{m}:\|x-\varphi(\hat{p})\| \leq \varepsilon\right\} \subset A$. Let $q \in B^{N}$ with $q(i)=\varphi(\hat{p})+$ $\frac{\varepsilon}{\|\hat{p}(i)-\varphi(\hat{p})\|}(\hat{p}(i)-\varphi(\hat{p}))$ for every $i \in N$. Then by positive association (see lemma 2.13), $\varphi(q)=\varphi(\hat{p})$.

Suppose $|\{q(i): i \in N\}|>2$. Then we can take different $x, y \in\{q(i)$ : $i \in N\}$ with $\varphi(q) \notin[x, y]$. Hence by strict convexity of $\|\cdot\|$ and lemma 2.1 (v), $\|x-y\|<2 \varepsilon$. Let $z=\frac{1}{2} x+\frac{1}{2} y$. Then $\|x-z\|<\varepsilon$ and $\|y-z\|<\varepsilon$. 
Let $\tilde{z}=\frac{1}{2} z+\frac{1}{2} \varphi(q)$. Then by lemma 2.1 (iii), $\|x-\tilde{z}\|<\varepsilon$ and $\|y-\tilde{z}\|<\varepsilon$. Let $r \in B^{N}$ be defined by $r(i)=\tilde{z}$ if $q(i) \in\{x, y\}$ and $r(i)=q(i)$ otherwise for every $i \in N$. Then by CSP, $\|x-\varphi(q)\| \leq\|x-\varphi(r)\|$ or $\|y-\varphi(q)\| \leq$ $\|y-\varphi(r)\|$. So $\varphi(r) \neq \tilde{z}$. Furthermore, by CSP, $\|\tilde{z}-\varphi(r)\| \leq\|\tilde{z}-\varphi(p)\|=$ $\left\|\frac{1}{4} x-\frac{1}{4} y-\frac{1}{2} \varphi(p)\right\|=\frac{1}{2}\left\|\frac{1}{2} x+\frac{1}{2} y-\varphi(p)\right\|<\frac{1}{2} \varepsilon$ because of lemma 2.1 (ii). Also, $\|\tilde{z}-r(i)\| \geq\|r(i)-\varphi(p)\|-\|\tilde{z}-r(i)\|>\varepsilon-\frac{1}{2} \varepsilon=\frac{1}{2} \varepsilon$ for all $i \in N$ with $r(i) \neq \tilde{z}$.

Consequently, $\varphi(r) \notin\{r(i): i \in N\}$. On the other hand $\mid\{r(i): i \in$ $N\}|<|\{q(i): i \in N\}|\leq|\{\hat{p}(i): i \in N\} \mid$, contradicting the definition of $\hat{p}$. Therefore, $|\{q(i): i \in N\}| \leq 2$. Let $v, w \in \mathbf{R}^{m}$ with $\{v, w\}=\{q(i): i \in N\}$. Let $V=\{i \in N: q(i)=v\}, W=N \backslash V$. Let $\psi: A^{2} \rightarrow A$ be defined by $\psi(x, y)=\varphi\left(\left\langle x^{V}, y^{W}\right\rangle\right)$ for all $x, y \in A$. Then by lemma $1.2, \psi$ is strategyproof with respect to $\|\cdot\|$. By the Pareto optimality of $\varphi, \psi$ is Pareto optimal with respect to $\|\cdot\|$. So by corollary $2.6, \varphi(q)=\varphi\left(\left\langle v^{V}, w^{W}\right\rangle\right)=\psi(v, w) \in$ $\{v, w\}=\{q(i): i \in N\}$. This contradicts the definition of $q$. Consequently, $\varphi(p) \in\{p(i): i \in N\}$.

Lemma 2.57 Let $A$ be a nonempty open subset of $\mathbf{R}^{m}, m \geq 2$. Let $\varphi$ : $A^{N} \rightarrow A$ satisfy PO and CSP with respect to strictly convex norm $\|\cdot\|$. Then there are $j \in N, u, v \in A$ with $u \neq v$ and $\varphi\left(\left\langle u^{\{j\}}, v^{N \backslash\{j\}}\right\rangle\right)=u$.

Proof W.l.o.g. assume that $n>1$. Since $A$ is nonempty and open, we can take $v \in A$ and $\left\{x \in \mathbf{R}^{m}:\|x-v\| \leq 2 \varepsilon\right\} \subset A$. Let $p \in A^{N}$ with all $p(i)$ different and $\|p(i)-v\|=\varepsilon$ for all $i \in N$. By lemma 2.56 we can take $j \in N$ such that $\varphi(p)=p(j)$. Let $u=v+2(p(j)-v)$. Let $q \in A^{N}$ be defined by $q(j)=u$ and $q={ }_{N \backslash\{j\}} p$. Then by SP, $\|q(j)-\varphi(q)\| \leq\|q(j)-p(j)\|=\varepsilon$. By the strict convexity of $\|\cdot\|$ and lemma 2.1 (v) this implies that $\varphi(q) \neq q(i)$ for all $i \in N \backslash\{j\}$. Hence by lemma 2.56, $\varphi(q)=u$. So $\|q(i)-v\|=$ $\varepsilon<\|q(i)-u\|$ for all $i \in N \backslash\{j\}$. Hence, by CSP, $\varphi\left(\left\langle u^{\{j\}}, v^{N \backslash\{j\}}\right\rangle\right) \neq v$. Consequently, lemma 2.56 implies that $\varphi\left(\left\langle u^{\{j\}}, v^{N \backslash\{j\}}\right\rangle\right)=u$.

Theorem 2.7 Let $A$ a nonempty open subset of $\mathbf{R}^{m}, m \geq 2$. Let $\varphi: A^{N} \rightarrow$ $A$ satisfy Pareto optimality and coalitional strategy-proofness with respect to a strictly convex norm $\|\cdot\|$. Then $\varphi$ is dictatorial.

Proof Lemma 2.57 implies that there are $j \in N, u, v \in A$ with $u \neq$ $v$ and $\varphi\left(\left\langle u^{\{j\}}, v^{N \backslash\{j\}}\right\rangle\right)=u$. Let $\psi: A^{2} \rightarrow A$ be defined by $\psi(x, y)=$ $\varphi\left(\left\langle x^{\{j\}}, y^{N \backslash\{j\}}\right\rangle\right)$ for all $x, y \in A$. Then by lemma $1.2 \psi$ satisfies strategyproofness with respect to $\|\cdot\|$. It is easy to verify that $\psi$ also satisfies Pareto optimality with respect to $\|\cdot\|$. So by corollary $2.6 \psi$ is dictatorial. Since 
$\psi(u, v)=u$, this implies that $\psi(x, y)=x$ for all $x, y \in A$, hence

$$
\varphi\left(\left\langle x^{\{j\}}, y^{N \backslash\{j\}}\right\rangle\right)=x \text { for all } x, y \in A \text {. }
$$

Let $p \in A^{N}$. Let $q=\left\langle p(j)^{\{j\}}, \varphi(p)^{N \backslash\{j\}}\right\rangle$. Then confirmatoriness implies that $\varphi(q)=\varphi(p)$. So by $(2.51), \varphi(p)=\varphi(q)=p(j)$. Since we can choose $p \in A^{N}$ arbitrarily, $\varphi$ is dictatorial.

Lemma 2.58 Let $A$ be a nonempty open subset of $\mathbb{R}^{m}$. Let $\varphi: A^{N} \rightarrow A$ satisfy SURJ, and CSP with respect to a strictly convex norm $\|\cdot\|$. Then $\varphi$ satisfies $P O$ with respect to $\|\cdot\|$.

Proof Suppose $\varphi$ does not satisfy PO with respect to $\|\cdot\|$. Then by proposition 2.1 we can take $p \in A^{N}, x \in A$ with $\|x-p(i)\|<\|\varphi(p)-p(i)\|$ for all $i \in N$. By SURJ we can take $q \in A^{N}$ with $x=\varphi(q)$. Then by CSP, $\|\varphi(p)-p(i)\| \leq\|x-p(i)\|$ for some $i \in N$. So we have a contradiction. Consequently, $\varphi$ satisfies PO with respect to $\|\cdot\|$.

The following corollary is a direct consequence of theorem 2.7 and lemma 2.58 .

Corollary 2.8 Let $A$ be a nonempty open subset of $\mathbb{R}^{m}, m \geq 2$. Let $\varphi$ : $A^{N} \rightarrow A$ satisfy surjectivity, and coalitional strategy-proofness with respect to a strictly convex norm. Then $\varphi$ is dictatorial.

Since unanimity implies surjectivity, we also have:

Corollary 2.9 Let $A$ be a nonempty open subset of $\mathbb{R}^{m}, m \geq 2$. Let $\varphi$ : $A^{N} \rightarrow A$ satisfy unanimity, and coalitional strategy-proofness with respect to a strictly convex norm. Then $\varphi$ is dictatorial.

Comparison of theorems 2.5 and 2.7 shows that coalitional strategy-proofness is far more restrictive than strategy-proofness. There are, however, conditions under which these axioms are equivalent. An example is the 1dimensional case (i.e., $A \subset \mathbf{R}$ ) with respect to single-peaked preferences, the case studied by Moulin (1980): then the mentioned equivalence holds, as is easily demonstrated. In the 1-agent case both axioms are equivalent by definition.

\subsection{Conclusions and open problems}

In section 2.2 we developed some theory that is mainly interesting from a mathematical point of view. There were three aspects in our research which 
seem to be neglected in the existing literature on orthogonality concepts with respect to norms:

(i) the definition of $\|\cdot\|$-orthogonality basically as a property of sets;

(ii) the existence of an $\|\cdot\|$-orthogonal basis;

(iii) the relation between $\|\cdot\|$-orthogonality and linear independence of a set of nonzero vectors.

From a decision theoretic viewpoint however, theorems 2.5 and 2.6 are the most important results of this chapter. It is relatively easy to see, that in these theorems SP and PO can be replaced by SSP and SPO. An open problem is whether these theorems will be valid for norms which are not strictly convex.

In order to derive theorem 2.5 we used anony mity. Another open question is what happens if we delete this axiom. In other words: what is the class of all voting schemes $\varphi:\left(\left(\mathbf{R}^{2}\right)^{N} \rightarrow \mathbb{R}^{2}\right.$ which satisfy SP and PO with respect to a strictly convex norm?

In section 2.8 we considered CSP with respect to a strictly convex norm $\|\cdot\|$. We conjecture that similar results can be obtained if $\|\cdot\|$ is not strictly convex. 



\section{Chapter 3}

\section{Pareto Optimality and Inner Product Metrics}

This chapter is based on Peters, Van der Stel and Storcken (1992a).

\subsection{Introduction}

In this chapter we study schemes $\varphi:\left(\mathbf{R}^{m}\right)^{N} \rightarrow \mathbf{R}^{m}$ which satisfy anonymity, Pareto optimality and strategy-proofness with respect to metrics $\delta$ which are induced by inner products. As all these metrics are strictly convex, we can use the results of chapter 2. One of the main objectives of this chapter is to give an alternative (and shorter) proof of theorem 2.5 for these metrics.

The organization of this proof is as follows. First we prove that Pareto optimality and strategy-proofness imply componentwise continuity. This new result is stated in proposition 3.1 and corollary 3.2 . We are then able to apply a result by Kim and Roush (1984), which holds for the case that $\delta$ is Euclidean. In this theorem (proposition 3.2 in this chapter) a characterization is given of all schemes which are anonymous, strategy-proof and componentwise continuous. All these schemes are coordinatewise median schemes with $n+1$ constant points. Corollary 3.4 is a generalization of this result for arbitrary inner product metrics. A characterization of all schemes which satisfy anonymity, and Pareto optimality and strategy-proofness with respect to an inner product metric is then easily obtained.

The organization of the chapter is as follows. In section 3.2 we focus attention on inner products on $\mathbf{R}^{m}$, and in particular on their relation with the standard dot product on $\mathbf{R}^{m}$. Next we study how a location problem with 
respect to an arbitrary metric which is induced by an inner product is related to the Euclidean case. Furthermore, an alternative characterization of Pareto optimality is given in lemma 3.4, which is comparable to results found earlier (cf. theorem 2.4). Section 3.3 is devoted to a proof of proposition 3.1 and corollary 3.2 , discussed earlier. Further main results are given in section 3.4. An important new impossibility result is given by theorem 3.2. This states that there are no schemes which are anonymous, and Pareto optimal and strategy-proof with respect to an inner product metric if $m \geq 3$ and $n \geq 2$. This result cannot be generalized for arbitrary norms, as is shown by example 2.13 . Section 3.5 concludes.

Since all metrics which are induced by an inner product are also induced by a strictly convex norm, we have by proposition 2.1 that with respect to such a metric PO and WPO are equivalent. This means that all the results of this chapter remain valid if we replace PO by WPO.

\subsection{Location problems and inner products}

Throughout this chapter we use the same notation as in the previous chapters. For this we refer to sections 1.2 and 2.2. For completeness we start with the definition of an inner product (cf. Kreyszig, 1978, pp. 128, 129): An inner product on $\mathbf{R}^{m}$ is a mapping from $\mathbf{R}^{m} \times \mathbf{R}^{m}$ into $\mathbf{R}$; that is, with every pair of vectors $x$ and $y$ there is associated a scalar which is written $\langle x, y\rangle$ and is called the inner product of $x$ and $y$, such that for all vectors $x, y, z$ and scalars $\alpha$ we have

(i) $\langle x+y, z\rangle=\langle x, z\rangle+\langle y, z\rangle$

(ii) $\langle\alpha x, y\rangle=\alpha\langle x, y\rangle$

(iii) $\langle x, y\rangle=\langle y, x\rangle$

(iv) $\langle x, x\rangle, \geq 0$

(v) $\langle x, x\rangle=0$ if and only if $x=0$.

Throughout this chapter we will assume the metric $\delta$ on $\mathbb{R}^{m}$ to be induced by the inner product $\langle\cdot, \cdot\rangle$ on $\mathbf{R}^{m}$, i.e. $\delta(x, y)=\sqrt{\langle x-y, x-y\rangle}$ for all $x, y \in \mathbb{R}^{m}$. Then $\delta$ is also induced by the norm $\|\cdot\|$, which is defined by $\|x\|=\sqrt{\langle x, x\rangle}$ for all $x \in \mathbf{R}^{m}$.

Lemma 3.1 Let the metric $\delta$ on $\mathbf{R}^{m}$ be induced by an inner product on $\mathbb{R}^{m}$. Then $\delta$ is strictly convex. 
Proof (For the sake of completeness we add this proof, which can also be found in Kreyszig, 1978, p. 333.)

Let $x, y \in \mathbb{R}^{m}$ with $\|x\| \leq 1,\|y\| \leq 1$ and $x \neq y$. Since $\|\cdot\|$ is induced by an inner product, we have:

$$
\|x+y\|^{2}=-\|x-y\|^{2}+2\left(\|x\|^{2}+\|y\|^{2}\right)
$$

(verification left to the reader).

So we have by assumption, that $\|x+y\|^{2}<2\left(\|x\|^{2}+\|y\|^{2}\right) \leq 4$. Consequently, $\|x+y\|<2$.

The best known inner product on $\mathbf{R}^{m}$ is the standard inner product on $\mathbb{R}^{m}$, which is defined by $x \cdot y=\sum_{j=1}^{m} x_{j} y_{j}$ for all $x, y \in \mathbb{R}^{m}$. It is easy to verify that this is indeed an inner product on $\mathbb{R}^{m}$. The Euclidean norm $\|\cdot\|_{2}$ is induced by the standard inner product, i.e. $\|\left. x\right|_{2}=\sqrt{\sum_{j=1}^{m} x_{j}^{2}}$ for all $x \in \mathbb{R}^{m}$. So by lemma 3.1, the Euclidean norm is strictly convex. Note that the Euclidean norm coincides with the $p$-norm with $p=2$. The Euclidean metric, denoted as $\delta_{2}$ is the metric induced by this norm, i.e. $\delta_{2}(x, y)=\sqrt{\sum_{j=1}^{m}\left(x_{j}-y_{j}\right)^{2}}$ for all $x, y \in \mathbb{R}^{m}$.

From elementary linear algebra it is known that all inner products on $\mathbf{R}^{m}$ are closely related. In particular we have the following lemma. (For completeness' sake, we include a proof.)

Lemma 3.2 Let $\langle\cdot$,$\rangle be an inner product on \mathbb{R}^{m}$. Then there is a nonsingular $m \times m$ matrix $B$ such that $\langle x, y\rangle=(B x) \cdot(B y)$ for all $x, y \in \mathbf{R}^{m}$.

Proof An inner product $\langle\cdot, \cdot\rangle$ on $\mathbf{R}^{m}$ is a positive definite symmetric bilinear form (see Lipschutz, 1974, p. 279), i.e., there is a symmetric positive definite $m \times m$ matrix $C$ such that $\langle x, y\rangle=x^{T} C y$ for all $x, y \in \mathbb{R}^{m}$. By the lemma of Sylvester, there is a nonsingular $m \times m$-matrix $P$ such that $I=P^{T} C P$. Hence, $C=B^{T} I B=B^{T} B$ with $B=P^{-1}$. So, $\langle x, y\rangle=x^{T} C y=x^{T} B^{T} B y=$ $(B x) \cdot(B y)$ for all $x, y \in \mathbf{R}^{m}$.

Consequently we have:

Corollary 3.1 Let $\delta$ be induced by an inner product on $\mathbf{R}^{m}$. Then there is a nonsingular $m \times m$ matrix $B$ such that $\delta(x, y)=\delta_{2}(B x, B y)$ for all $x, y \in \mathbf{R}^{m}$.

The following lemma extends theorem 2.1 to higher dimensions, under the assumptions that the used norm is induced by an inner product and $A=\mathbf{R}^{m}$. 
Lemma 3.3 Let $\delta$ be induced by an inner product $\langle\cdot, \cdot\rangle$ on $\mathbb{R}^{m}$. Then a scheme $\varphi:\left(\mathbf{R}^{m}\right)^{N} \rightarrow \mathbf{R}^{m}$ is Pareto optimal with respect to $\delta$ if and only if $\varphi(p)$ is in the convex $h u l l$ of $\{p(i): i \in N\}$ for every profile $p$.

Proof First we prove the if-part. Let

$$
\varphi(p) \in \operatorname{conv}\{p(i): i \in N\} \text { for all } p \in\left(\mathbb{R}^{m}\right)^{N} .
$$

Let $q \in\left(\mathbb{R}^{m}\right)^{N}, y \in \mathbb{R}^{m}$ with $\delta(y, q(i)) \leq \delta(\varphi(q), q(i))$ for all $i \in N$. Let $w=\varphi(q)$. Then:

$$
\langle y-q(i), y-q(i)\rangle \leq\langle w-q(i), w-q(i)\rangle \text { for all } i \in N .
$$

Since by (3.1), $w \in \operatorname{conv}\{q(i): i \in N\}$, we can take $\lambda_{i} \in[0,1], i \in N$ such that:

$$
\sum_{i \in N} \lambda_{i}=1 \text { and } w=\sum_{i \in N} \lambda_{i} q(i)
$$

(cf. Rockafellar, 1970, p. 11). From (3.2) we derive that for all $i \in N$,

$$
\langle y, y\rangle-2\langle q(i), y\rangle-\langle w, w\rangle+2\langle q(i), w\rangle \leq 0 \text {. }
$$

Hence,

$$
\sum_{i \in N} \lambda_{i}(\langle y, y\rangle-2\langle q(i), y\rangle-\langle w, w\rangle+2\langle q(i), w\rangle) \leq 0
$$

So,

$$
\sum_{i \in N} \lambda_{i}\langle y, y\rangle-2 \sum_{i \in N}\left\langle\lambda_{i} q(i), y\right\rangle-\sum_{i \in N} \lambda_{i}\langle w, w\rangle+2 \sum_{i \in N}\left\langle\lambda_{i} q(i), w\right\rangle \leq 0
$$

Hence, by (3.3), $\langle y, y\rangle-2\langle w, y\rangle-\langle w, w\rangle+2\langle w, w\rangle \leq 0$. So $\langle y-w, y-w\rangle \leq 0$. Hence, $y=w$. Consequently, $\varphi$ is Pareto optimal with respect to $\delta$. This completes the if-part of the proof.

Now assume $\varphi$ is Pareto optimal with respect to $\delta$. Suppose $\tilde{p} \in\left(\mathbb{R}^{m}\right)^{N}$ is such that $\varphi(\tilde{p}) \notin \operatorname{conv}\{\tilde{p}(i): i \in N\}$. Let $\tilde{w}=\varphi(\tilde{p})$. By compactness we can define $\tilde{z} \in \operatorname{conv}\{\tilde{p}(i): i \in N\}$ such that $\delta(\tilde{w}, \tilde{z})$ is minimal. Since also $\tilde{z}+\lambda(\tilde{p}(i)-\tilde{z}) \in \operatorname{conv}\{\tilde{p}(i): i \in N\}$ for all $\lambda \in[0,1], i \in N$, this implies that $\delta(\tilde{w}, \tilde{z}) \leq \delta(\tilde{w}, \tilde{z}+\lambda(\tilde{p}(i)-\tilde{z}))$ for all $\lambda \in[0,1], i \in N$. Hence, $\langle\tilde{w}-\tilde{z}, \tilde{w}-\tilde{z}\rangle \leq\langle\tilde{w}-\tilde{z}-\lambda(\tilde{p}(i)-\tilde{z}), \tilde{w}-\tilde{z}-\lambda(\tilde{p}(i)-\tilde{z})\rangle$ for all $\lambda \in[0,1]$, $i \in N$. So,

$$
0 \leq-2 \lambda\langle\tilde{p}(i)-\tilde{z}, \tilde{w}-\tilde{z}\rangle+\lambda^{2}\langle p(i)-\tilde{z}, p(i)-\tilde{z}\rangle \text { for all } \lambda \in[0,1], i \in N
$$


This is only possible, if $\langle\tilde{p}(i)-\tilde{z}, \tilde{w}-\tilde{z}\rangle \leq 0$ for all $i \in N$. Hence, $\langle\tilde{p}(i)-\tilde{z}, \tilde{z}-\tilde{w}\rangle \geq 0$ for all $i \in N$. So,

$$
\begin{aligned}
\langle\tilde{p}(i)-\tilde{w}, \tilde{p}(i)-\tilde{w}\rangle= & \langle\tilde{p}(i)-\tilde{z}+\tilde{z}-\tilde{w}, \tilde{p}(i)-\tilde{z}+\tilde{z}-\tilde{w}\rangle \\
= & \langle\tilde{p}(i)-\tilde{z}, \tilde{p}(i)-\tilde{z}\rangle+2\langle\tilde{p}(i)-\tilde{z}, \tilde{z}-\tilde{w}\rangle \\
& +\langle\tilde{z}-\tilde{w}, \tilde{z}-\tilde{w}\rangle \\
> & \langle\tilde{p}(i)-\tilde{z}, \tilde{p}(i)-\tilde{z}\rangle \text { for all } i \in N .
\end{aligned}
$$

Hence, $\delta(\tilde{p}(i), \tilde{z})<\delta(\tilde{p}(i), \tilde{w})$ for all $i \in N$. This contradicts the Pareto optimality of $\varphi$. Consequently, $\varphi(\tilde{p}) \in \operatorname{conv}\{\tilde{p}(i): i \in N\}$ for all $\tilde{p} \in\left(\mathbf{R}^{m}\right)^{N}$.

In lemma 3.3 the assumption that $\delta$ is induced by an inner product seems to be essential. Anyway, it is not sufficient to assume only that $\delta$ is strictly convex, as is shown by example 2.10 . In this example we describe a strictly convex norm with respect to which Pareto optimality does not imply that the compromise point is always in the convex hull of the reported points. We even suspect:

Conjecture 3.1 Let $m \geq 3, n \geq 3$. Let $A$ be a nonempty open subset of $\mathbf{R}^{m}$. Let $\|\cdot\|$ be a norm on $\mathbb{R}^{m}$ which is not induced by an inner product on $\mathrm{R}^{m}$. Then there is a voting scheme $\varphi: A^{N} \rightarrow A$ which satisfies SPO with respect to $\|\cdot\|$ such that $\varphi(p) \notin \operatorname{conv}\{p(i): i \in N\}$ for some $p \in A^{N}$.

Lemma 3.4 extends corollary 2.5 for the case that the norm under consideration is induced by an inner product.

Lemma 3.4 Let $\delta$ be induced by an inner product on $\mathbb{R}^{m}$. Let $U=\left\{u^{j}\right.$ : $j=1, \ldots, m\}$ be a basis of $\mathbf{R}^{m}$. Let $\varphi:\left(\mathbf{R}^{m}\right)^{N} \rightarrow \mathbb{R}^{m}$ be a coordinatewise median scheme with respect to $U$ without constant points, $n \geq 2$. Then $\varphi$ is Pareto optimal with respect to $\delta$ if and only if $m \in\{1,2\}$.

Proof For $m=1$ the Pareto optimality of $\varphi$ is trivial. Now assume $m \geq 2$. Since by lemma $3.1 \delta$ is strictly convex, we can use the results of chapter 2 . If $m=2$, then by lemma $2.20, \varphi$ is Pareto optimal with respect to $\delta$. Next, let $m>2$. Since $\varphi$ is a coordinatewise median scheme without constant points, $n$ is odd. Hence we can define $k \in\{1,2,3, \ldots\}$ such that $n=2 k+1$. Let $J, K, L \subset N$ be such that $J \cup K \cup L=N,|J|=k,|K|=k$ and $|L|=1$. Then $\varphi\left(\left\langle\left(u^{1}\right)^{J},\left(u^{2}\right)^{K},\left(u^{3}\right)^{L}\right\rangle\right)=0 \notin \Delta\left(u^{1}, u^{2}, u^{3}\right)$. So by lemma $3.3, \varphi$ is not Pareto optimal with respect to $\delta$. 
We conjecture that in lemma 3.4 the restriction that $\delta$ is induced by an inner product can be relaxed. As we are not mainly focussed on coordinatewise median schemes, we leave this issue to the reader.

We have already remarked (cf. corollary 3.1 ) that all inner product metrics on $\mathbf{R}^{\boldsymbol{n}}$ are closely related: each such metric is a transformation of the Euclidean metric $\delta_{2}$. Voting schemes can be transformed accordingly, preserving properties like strategy-proofness, Pareto optimality and range convexity (cf. lemmas 1.5 and 1.6 ):

Lemma 3.5 Let $\delta$ be induced by an inner product on $\mathbb{R}^{m}$. Let $B$ be a nonsingular $m \times m$ matrix with $\delta(x, y)=\delta_{2}(B x, B y)$ for every $x, y \in \mathbb{R}^{m}$. Then:

(i) $\varphi$ satisfies $S P$ with respect to $\delta$ if and only if $B \circ \varphi \circ B^{-1}$ satisfies $S P$ with respect to $\delta_{2}$.

(ii) $\varphi$ satisfies $P O$ with respect to $\delta$ if and only if $B \circ \varphi \circ B^{-1}$ satisfies $P O$ with respect to $\delta_{2}$.

(iii) $\varphi$ satisfies $R C$ if and only if $B \circ \varphi \circ B^{-1}$ satisfies $R C$.

\section{Proof}

(i) We have that:

$\varphi$ satisfies SP with respect to $\delta$

$\Leftrightarrow \delta(p(i), \varphi(p)) \leq \delta(p(i), \varphi(\hat{p}))$ for all $i \in N, p, \hat{p} \in\left(\mathbf{R}^{m}\right)^{N}$ with $\hat{p}=N \backslash\{i\} p$

$\Leftrightarrow \delta_{2}(B p(i), B \varphi(p)) \leq \delta_{2}(B p(i), B \varphi(\hat{p}))$ for all $i \in N, p, \hat{p} \in\left(\mathbf{R}^{m}\right)^{N}$ with $\hat{p}=N \backslash\{i\} p$

$\Leftrightarrow \delta_{2}\left(B B^{-1} q(i), B \varphi\left(B^{-1} q\right)\right) \leq \delta_{2}\left(B B^{-1} q(i), B \varphi\left(B^{-1} q\right)\right)$ for all $i \epsilon$ $N, q, \hat{q} \in\left(\mathbf{R}^{m}\right)^{N}$ with $\hat{q}={ }_{N \backslash\{i\}} q$

$\Leftrightarrow \delta_{2}\left(q(i), B \varphi\left(B^{-1} q\right)\right) \leq \delta_{2}\left(q(i), B \varphi\left(B^{-1} q\right)\right)$ for all $i \in N, q, \hat{q} \in$ $\left(\mathbb{R}^{m}\right)^{N}$ with $\hat{q}=N \backslash\{i\} q$

$\Leftrightarrow B \circ \varphi \circ B^{-1}$ satisfies SP with respect to $\delta$.

(ii) We have that:

$\varphi$ is not Pareto optimal with respect to $\delta$

$\Leftrightarrow$ There are $x \in \mathbb{R}^{m}, p \in\left(\mathbb{R}^{m}\right)^{N}, j \in N$ such that $\delta(x, p(i)) \leq$ $\delta(\varphi(p), p(i))$ for all $i \in N$ and $\delta(x, p(j))<\delta(\varphi(p), p(j))$ 
$\Leftrightarrow$ There are $x \in \mathbf{R}^{m}, p \in\left(\mathbf{R}^{m}\right)^{N}, j \in N$ such that $\delta_{2}(B x, B p(i)) \leq$ $\delta_{2}(B \varphi(p), B p(i))$ for all $i \in N$ and $\delta_{2}(B x, B p(j))<\delta_{2}(B \varphi(p)$, $B p(j))$

$\Leftrightarrow$ (Take $y=B x$ and $q=B p$, so $p=B^{-1} q$.) There are $y \in \mathbf{R}^{m}, q \in$ $\left(\mathbb{R}^{m}\right)^{N}, j \in N$ such that $\delta_{2}(y, q(i)) \leq \delta_{2}\left(B \varphi\left(B^{-1} q\right), q\right)$ for all $i \in N$ and $\delta_{2}(y, q(j))<\delta_{2}\left(B \varphi\left(B^{-1} q\right), q(j)\right)$

$\Leftrightarrow B \circ \varphi \circ B^{-1}$ is not Pareto optimal with respect to $\delta_{2}$.

(iii) We have that:

$\varphi$ satisfies $\mathrm{RC}$

$\Leftrightarrow \varphi\left(\left(\mathbf{R}^{m}\right)^{N}\right)$ is a convex set

$\Leftrightarrow \varphi\left(\left(B^{-1} \circ \mathbf{R}^{m}\right)^{N}\right)$ is a convex set (note that $B^{-1} \circ \mathbf{R}^{m}=\mathbf{R}^{m}$ )

$\Leftrightarrow \varphi \circ B^{-1}\left(\left(\mathbf{R}^{m}\right)^{N}\right)$ is a convex set

$\Leftrightarrow B \circ \varphi \circ B^{-1}\left(\left(\mathbb{R}^{m}\right)^{N}\right)$ is a convex set

$\Leftrightarrow B \circ \varphi \circ B^{-1}$ satisfies RC.

\subsection{Pareto optimality and strategy-proofness in $\mathrm{IR}^{m}$}

In this section we assume the metric $\delta$ on $\mathbf{R}^{m}$ to be induced by a strictly convex norm. At the end of the section (proposition 3.1 and corollary 3.2) we focus on metrics which are induced by an inner product $\langle\cdot, \cdot\rangle$ on $\mathbf{R}^{m}$. We study schemes which satisfy Pareto optimality and strategy-proofness with respect to such a metric. The main result is given in proposition 3.1 and corollary 3.2 , which state that all such schemes are componentwise continuous. We use the following notation: $x^{i} \rightarrow x$ means that $\lim _{i \rightarrow \infty} x^{i}=x$. A limit point of a sequence is the limit of some converging subsequence.

Lemmas 3.6-3.9 are used in the proof of proposition 3.1. This proposition states that all schemes $\varphi:\left(\mathbb{R}^{m}\right)^{N} \rightarrow \mathbb{R}^{m}$ which satisfy PO and SP with respect to the Euclidean metric $\delta_{2}$ must be componentwise continuous. It suffices to prove that such a $\varphi$ is continuous in the first component (the proof that $\varphi$ is continuous in the other components is analogous). For this reason we study, in lemmas 3.6-3.8, sequences of profiles $\left\{p^{i}\right\}(i \in \mathbb{N})$ which only differ from each other in the first component, whereas $p^{i}(1)$ converges to $p^{0}(1)$. We focus on properties of the sequence $\left\{\varphi\left(p^{i}\right)\right\}$, which will be denoted as $\left\{w^{i}\right\}$ in lemmas 3.6-3.8. Note further that in these lemmas only strategy-proofness is assumed.

Lemma 3.6 states that: 
(i) $\left\{w^{i}\right\}$ must have a convergent subsequence,

(ii) $\left\{w^{i}\right\}$ converges to $y$ if and only if $y$ is the only limit point of $w^{i}$,

(iii) every limit point of $\left\{w^{i}\right\}$ has the same distance to $p(1)$ as $w^{0}$ has.

Lemma 3.6 Let $A$ be a nonempty subset of $\mathbf{R}^{m}$. Let $\delta$ be induced by a norm on $\mathbf{R}^{m}$. Let $\varphi: A^{N} \rightarrow A$ be strategy-proof with respect to $\delta$. Let $y, v, x^{i} \in A,(i \in \mathbb{N} \cup\{0\})$ with $x^{i} \rightarrow x^{0}, p \in A^{N \backslash\{1\}}$. For every $i \in \mathbb{N} \cup\{0\}$ let $w^{i}:=\varphi\left(x^{i}, p\right)$. Then:

(i) $\left\{w^{i}: i \in \mathbb{N}\right\}$ has a limit point in $\mathbb{R}^{m}$,

(ii) $w^{i} \rightarrow y \Leftrightarrow$ if $z$ is a limit point of $\left\{w^{i}: i \in \mathbb{N}\right\}$ then $z=y$,

(iii) if $v$ is a limit point of $\left\{w^{i}: i \in \mathbb{N}\right\}$ then $\delta\left(x^{0}, w^{0}\right)=\delta\left(x^{0}, v\right)$.

Proof (i) We prove that there is a compact set $W$ with $\left\{w^{i}: i \in \mathbb{N}\right\} \subset W$. By strategy-proofness, $\delta\left(x^{i}, w^{i}\right) \leq \delta\left(x^{i}, w^{0}\right)$ for all $i \in \mathbf{N}$. Hence $\delta\left(x^{0}, x^{i}\right)+$ $\delta\left(x^{i}, w^{i}\right) \leq \delta\left(x^{0}, x^{i}\right)+\delta\left(x^{i}, w^{0}\right)$. So, by applying two triangular inequalities, $\delta\left(x^{0}, w^{i}\right) \leq 2 \delta\left(x^{0}, x^{i}\right)+\delta\left(x^{0}, w^{0}\right)$. Because $x^{i} \rightarrow x^{0}$, there is a $\lambda>0$ such that $\delta\left(x^{0}, w^{i}\right) \leq \lambda$ for all $i \in \mathbf{N}$. This implies the existence of a set $W$ as above.

(ii) " $\Rightarrow "$ is trivial. For the converse, suppose $w^{i} \not y$. Then there is an $\varepsilon>0$ and a subsequence of $\left\{w^{i}: i \in \mathbb{N}\right\}$, say $\left\{\tilde{w}^{j}: j \in \mathbb{N}\right\}$, with $\delta\left(\tilde{w}^{j}, y\right)>\varepsilon$. By (i), $\left\{\tilde{w}^{j}: j \in \mathbb{N}\right\}$ has a limit point unequal to $y$. So $\left\{w^{i}: i \in \mathbf{N}\right\}$ has a limit point unequal to $y$. This completes the proof of (ii).

(iii) Follows directly from lemma 2.16.

Lemma 3.7 establishes a kind of positive association for sequences of profiles. It implies that if some of the reported points of the agents $2, \ldots, n$ are moved in the direction of a limit point of $\left\{w^{i}\right\}$, this remains to be a limit point.

Lemma 3.7 Let $\delta$ be induced by a strictly convex norm on $\mathbb{R}^{m}$. Let $\varphi$ : $\left(\mathbf{R}^{m}\right)^{N} \rightarrow \mathbf{R}^{m}$ be strategy-proof with respect to $\delta$. Let $y, z, v, x^{i} \in \mathbf{R}^{m}$ (i $\in$ $\mathbf{N} \cup\{0\})$ with $x^{i} \rightarrow x^{0}$, and $p, q \in\left(\mathbb{R}^{m}\right)^{N \backslash\{1\}}$ such that $q(j)=p(j)$ if $p(j) \neq y$ and $q(j)=z$ if $p(j)=y$, for all $j \in \mathbb{N} \backslash\{1\}$. Let $w^{i}:=\varphi\left(x^{i}, p\right)$ and $v^{i}:=\varphi\left(x^{i}, q\right)$ for all $i \in \mathbb{N}, w^{i} \rightarrow v$ and $z \in[y, v]$. Then $v^{i} \rightarrow v$.

Proof In view of lemma 3.6 (i), $\left\{v^{i}: i \in \mathbf{N}\right\}$ has a limit point, say $u$. By lemma 3.6 (ii) it is sufficient to prove that $u=v$. There are subsequences $\left\{\tilde{x}^{i}: i \in \mathbb{N}\right\}$ of $\left\{x^{i}: i \in \mathbf{N}\right\}$ and $\left\{\tilde{v}^{i}: i \in \mathbb{N}\right\}$ of $\left\{v^{i}: i \in \mathbb{N}\right\}$ with 
$\varphi\left(\tilde{x}^{i}, q\right)=: \tilde{v}^{i} \rightarrow u$. Let $\tilde{w}^{i}:=\varphi\left(\tilde{x}^{i}, p\right)$, so $\tilde{w}^{i} \rightarrow v$. By strategy-proofness and lemma $1.2, \delta\left(y, \tilde{w}^{i}\right) \leq \delta\left(y, \tilde{v}^{i}\right)$ and $\delta\left(z, \tilde{v}^{i}\right) \leq \delta\left(z, \tilde{w}^{i}\right)$ for all $i \in \mathbb{N}$. Hence, $\delta(y, v) \leq \delta(y, u)$ and $\delta(z, u) \leq \delta(z, v)$ for all $i \in \mathbb{N}$. Because $z \in[y, v]$, it follows that $v=u$.

Lemma 3.8 states that no one of the agents $j \in\{2, \ldots, n\}$ can enforce $w^{0}$ to be the only limit point of $\left\{w^{i}\right\}$ if $w^{0}$ is closer to his reported point than some of the limit points of $\left\{w^{i}\right\}$, by reporting $w^{0}$ instead of $p(j)$.

Lemma 3.8 Let $\delta$ be induced by a strictly convex norm on $\mathbb{R}^{m}$. Let $\varphi$ : $\left(\mathbb{R}^{m}\right)^{N} \rightarrow \mathbb{R}^{m}$ be strategy-proof with respect to $\delta$. Let $x^{i} \in \mathbb{R}^{m}(i \in \mathbb{N} \cup\{0\})$ with $x^{i} \rightarrow x^{0}, p, q \in\left(\mathbb{R}^{m}\right)^{N \backslash\{1\}}$, and $w^{i}:=\varphi\left(x^{i}, p\right)$ for all $i \in \mathbb{N} \cup\{0\}$. Let $u$ be a limit point of $\left\{w^{i}: i \in \mathbb{N}\right\}$ with $u \neq w^{0}$. Let $k \in N \backslash\{1\}$ with $\delta\left(p(k), w^{0}\right)<\delta(p(k), u)$. Let $q(j)=p(j)$ for $j \neq k$ and let $q(k)=w^{0}$. Let $v^{i}:=\varphi\left(x^{i}, q\right)$ for all $i \in \mathbf{N}$. Then $\varphi\left(x^{0}, q\right)=w^{0}$ and $v^{i} \nrightarrow w^{0}$.

Proof Because $u$ is a limit point of $\left\{w^{i}: i \in \mathbb{N}\right\}$ it is w.l.o.g. to assume that $w^{i} \rightarrow u$ (otherwise, take a subsequence). By lemma 3.6 (i) $\left\{v^{i}: i \in \mathbf{N}\right\}$ has a limit point, say $v$. By strategy-proofness, $\delta\left(p(k), w^{i}\right) \leq \delta\left(p(k), v^{i}\right)$ for all $i \in \mathbb{N}$. Hence $\delta(p(k), u) \leq \delta(p(k), v)$. So $\delta\left(p(k), w^{0}\right)<\delta(p(k), v)$, which implies $v \neq w^{0}$. Therefore, by lemma 3.6 (ii), $v^{i} \nrightarrow w^{0}$. The other statement, $\varphi\left(x^{0}, q\right)=w^{0}$, is a direct consequence of confirmatoriness (see lemma 1.4).

If some agents all choose the same point on a line segment, there is a choice which has maximal distance to the resulting compromise point.

Lemma 3.9 Let $\delta$ be induced by a strictly convex norm on $\mathbb{R}^{m}$. Let $\varphi$ : $\left(\mathbb{R}^{m}\right)^{N} \rightarrow \mathbb{R}^{m}$ be strategy-proof with respect to $\delta$. Let $S \subset N, y, z \in \mathbf{R}^{m}$, and $p \in\left(\mathbf{R}^{m}\right)^{N \backslash S}$. Then there is a $u \in[y, z]$ for which $\delta\left(u, \varphi\left(u^{S}, p\right)\right)$ is maximal on $[y, z]$.

Proof Let $f: x \mapsto \delta\left(x, \varphi\left(x^{\mathcal{S}}, p\right)\right)$ on $[y, z]$. Lemma 2.16 implies that $f$ is continuous on $[y, z]$, which is a compact set. Consequently, $f$ has a maximum on $[y, z]$.

We now focus on inner product metrics. For the Euclidean metric $\delta_{2}$ we have:

Proposition 3.1 Let $\varphi:\left(\mathbf{R}^{m}\right)^{N} \rightarrow \mathbb{R}^{m}$ be strategy-proof and Pareto optimal with respect to $\delta_{2}$. Then $\varphi$ is componentwise continuous. 
Proof Lemma 3.1 implies that $\delta_{2}$ is induced by a strictly convex norm. Hence lemmas 3.6 to 3.9 can be applied. Let $x^{i} \rightarrow x^{0}, p \in\left(\mathbf{R}^{m}\right)^{N \backslash\{1\}}$, $w^{i}=\varphi\left(x^{i}, p\right)$ for all $i \in \mathbf{N} \cup\{0\}$. It is sufficient to prove $w^{i} \rightarrow w^{0}$. We assume $w^{i} \nrightarrow w^{0}$ and derive a contradiction. By lemma 3.6 (iii) there is a limit point $v$ of $\left\{w^{i}: i \in \mathbf{N}\right\}$ with $v \neq w^{0}$. W.l.o.g., assume $w^{i} \rightarrow v$ (otherwise take a subsequence). By lemma 3.8 we may assume w.l.o.g. that

$$
\delta_{2}(p(j), v) \leq \delta_{2}\left(p(j), w^{0}\right) \text { for all } j \in N \backslash\{1\} \text { with } p(j) \neq w^{0} .
$$

By lemma 3.6 (iii),

$$
\delta_{2}\left(x^{0}, w^{0}\right)=\delta_{2}\left(x^{0}, v\right) .
$$

(See figure 3.1.) Let $S=\left\{j \in N \backslash\{1\}: p(j)=w^{0}\right\}$. By lemma 3.3,

$$
w^{0} \in \operatorname{conv}\left\{x^{0}, p(2), \ldots, p(n)\right\} .
$$

So by (3.4) and (3.5), $S \neq \emptyset$.

Let $y=(4 / 5) w^{0}+(1 / 5) v$. Let $g: x \mapsto \varphi\left(x^{0}, x^{S},\left.p\right|_{N \backslash(S \cup\{1\})}\right)$. By lemma 3.9 there is a $z \in\left[w^{0}, y\right]$ for which $\delta_{2}(z, g(z))$ is maximal on $\left[w^{0}, y\right]$. From lemma 3.7, $\varphi\left(x^{i}, \tilde{z}^{S},\left.p\right|_{N \backslash(S \cup\{1\})}\right) \rightarrow v$ for all $\tilde{z} \in\left[w^{0}, y\right]$. So by lemma 3.6 (iii),

$$
\delta_{2}\left(x^{0}, v\right)=\delta_{2}\left(x^{0}, g(\tilde{z})\right) \text { for all } \tilde{z} \in\left[w^{0}, y\right] .
$$

In particular, $\delta_{2}\left(x^{0}, v\right)=\delta_{2}\left(x^{0}, g(y)\right)$. Therefore, $y \neq g(y)$ for otherwise, by (3.5), $\delta_{2}\left(x^{0}, y\right)=\delta_{2}\left(x^{0}, v\right)=\delta_{2}\left(x^{0}, w^{0}\right)$ which is impossible by definition of $y$ and the strict convexity of $\delta_{2}$. Consequently, by definition of $z, z \neq g(z)$. Because $w^{0}=\varphi\left(x^{0}, p\right)=g\left(w^{0}\right)$, we must have $z \neq w^{0}$. Further, by strategyproofness,

$$
\delta_{2}(z, g(z)) \leq \delta_{2}\left(z, w^{0}\right)
$$

Let $z^{i} \rightarrow z$ with $z^{i} \in\left(z, w^{0}\right)$ for all $i \in \mathbf{N}$. By (3.7),

$$
\delta_{2}\left(x^{0}, g\left(z^{i}\right)\right)=\delta_{2}\left(x^{0}, v\right) \text { for all } i \in \mathbb{N} \text {. }
$$

Suppose that for some $k \in \mathbf{N}, \angle\left(w^{0}, z, g\left(z^{k}\right)\right)>90^{\circ}$. Then $\delta_{2}\left(z, g\left(z^{k}\right)\right)<$ $\delta_{2}\left(z^{k}, g\left(z^{k}\right)\right)$ and by strategy-proofness, $\delta_{2}(z, g(z))<\delta_{2}\left(z, g\left(z^{k}\right)\right)$. So $\delta_{2}(z$, $g(z))<\delta_{2}\left(z^{k}, g\left(z^{k}\right)\right)$, contradicting the maximality of $\delta_{2}(z, g(z))$ on $\left[w^{0}, y\right]$. Hence,

$$
\angle\left(w^{0}, z, g\left(z^{i}\right)\right) \leq 90^{\circ} \text { for all } i \in \mathbf{N} .
$$

By (3.4), (3.5) and lemma 3.3,

$$
\angle\left(w^{0}, z^{i}, g\left(z^{i}\right)\right)>90^{\circ} \text { for all } i \in \mathbf{N} \text {. }
$$




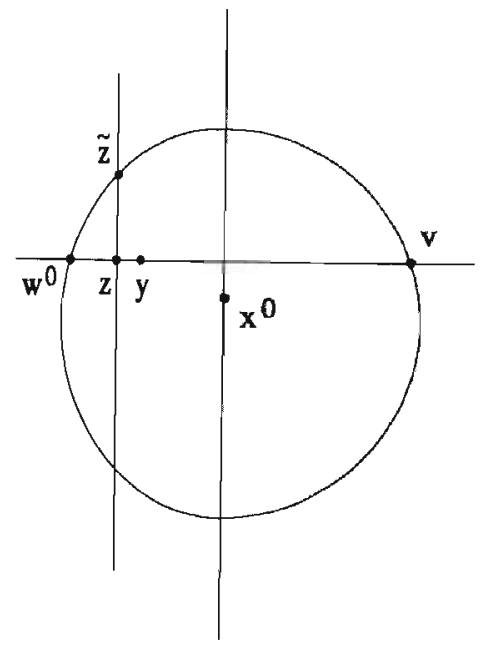

Figure 3.1: Proof of proposition 3.1

By (3.8), w.l.o.g. assume $\left\{g\left(z^{i}\right)\right\}$ converges, say to $\tilde{z} \neq z$. By (3.9) and (3.10), $\angle\left(w^{0}, z, \tilde{z}\right)=90^{\circ}$.

By (3.4) and (3.5), there is an $\varepsilon>0$ such that $\delta_{2}(\tilde{z}, x)>\varepsilon$ for all $x \in$ $\operatorname{conv}\left\{x^{0}, a, p(j): j \in N \backslash(S \cup\{1\})\right\}$ and $a \in\left[z, w^{0}\right]$ close enough to $z$. Consequently, for $k$ large enough, $g\left(z^{k}\right) \notin \operatorname{conv}\left\{x^{0}, z^{k}, p(j): j \in N \backslash(S \cup\{1\})\right\}$. This contradicts Pareto optimality and completes the proof.

Proposition 3.1 can easily be generalized to the whole class of metrics which are induced by an inner product:

Corollary 3.2 Let $\delta$ be induced by an inner product on $\mathbf{R}^{m}$. Let $\varphi$ : $\left(\mathbb{R}^{m}\right)^{N} \rightarrow \mathbb{R}^{m}$ be strategy-proof and Pareto optimal with respect to $\delta$. Then $\varphi$ is componentwise continuous.

Proof By corollary 3.1 there is a nonsingular $m \times m$ matrix $B$ with $\delta(x, y)=\delta_{2}(B x, B y)$ for all $x, y \in \mathbb{R}^{m}$. Let $\psi:\left(\mathbb{R}^{m}\right)^{N} \rightarrow \mathbb{R}^{m}$ be defined by $\psi=B \circ \varphi \circ B^{-1}$. Then by lemma $3.5, \psi$ satisfies SP and PO with respect to $\delta_{2}$. So by proposition $3.1, \psi$ is componentwise continuous. Since $\varphi=B^{-1} \circ \varphi \circ B$, this implies that $\varphi$ is componentwise continuous. 
We conjecture that this result is not only valid for the class of norms which are induced by an inner product, but for the whole class of strictly convex norms:

Conjecture 3.2 Let $\delta$ be induced by a strictly convex norm on $\mathbf{R}^{m}$. Let $\varphi:\left(\mathbf{R}^{m}\right)^{N} \rightarrow \mathbb{R}^{m}$ be strategy-proof and Pareto optimal with respect to $\delta$. Then $\varphi$ is componentwise continuous.

So far, this conjecture has been proved for the cases $n=1$ (trivial), $n=2$ (then the continuity follows from corollary 2.6, which states that $\varphi$ must be dictatorial) and $n=3$ (lemma 2.36).

Proposition 3.1 and corollary 3.2 are very similar to theorem 4.2 which also establishes componentwise continuity. The difference is, that in theorem 4.2 range convexity is used in stead of Pareto optimality. Due to the fact that $\mathrm{RC}$ is much weaker than $\mathrm{PO}$, we succeeded only to prove theorem 4.2 for the case $m=2$ (cf. conjecture 4.2).

\subsection{Further main results}

In this section we use a result from Kim and Roush (1984), which enables us to give an alternative proof for corollary 2.7 and theorems 2.5 and 2.6 for inner product metrics. Finally we arrive at an impossibility result for $m \geq 3$, which does not hold for arbitrary strictly convex norms.

The following result will play an important role in this section:

Proposition 3.2 (Kim and Roush, 1984, Theorem 97.) Let $n \geq 2$. Let $\delta_{2}$ be the Euclidean metric on $\mathbb{R}^{2}$. Then a voting scheme $\varphi:\left(\mathbb{R}^{2}\right)^{N} \rightarrow \mathbf{R}^{2}$ is anonymous, componentwise continuous, and strategy-proof with respect to $\delta_{2}$, if and only if there are nonzero $u, v \in \mathbf{R}^{2}$ and $c^{1}, \ldots, c^{n+1} \in \overline{\mathbf{R}}^{2}$ with $u \cdot v=0$ and $\varphi$ being the coordinatewise median scheme with respect to $\{u, v\}$ with constant points $c^{1}, \ldots, c^{n+1}$.

This result can easily be generalized for arbitrary metrics which are induced by an inner product.

Corollary 3.3 Let $n \geq 2$. Let the metric $\delta$ on $\mathbf{R}^{2}$ be induced by an inner product $\langle\cdot, \cdot\rangle$ on $\mathbf{R}^{2}$. Then a scheme $\varphi: \mathbb{R}^{2} \rightarrow \mathbb{R}^{2}$ is anonymous, componentwise continuous, and strategy-proof with respect to $\delta$, if and only if there are nonzero $u, v \in \mathbf{R}^{2}$ and $c^{1}, \ldots, c^{n+1} \in \overline{\mathbf{R}}^{2}$ with $\langle u, v\rangle=0$ and $\varphi$ being the coordinatewise median scheme with respect to $\{u, v\}$ with constant points $c^{1}, \ldots, c^{n+1}$. 
Proof By lemma 3.2 there is a nonsingular $2 \times 2$ matrix $B$ such that $\langle x, y\rangle=(B x) \cdot(B y)$ for all $x, y \in \mathbb{R}^{2}$.

(only if) Let $\varphi:\left(\mathbf{R}^{2}\right)^{N} \rightarrow \mathbf{R}^{2}$ be anonymous, strategy-proof with respect to $\delta$ and componentwise continuous. Let $\psi=B \circ \varphi \circ B^{-1}$. Then by lemma $3.5, \psi$ satisfies SP with respect to $\delta_{2}$. Of course, $\psi$ is also anonymous and componentwise continuous.

So by proposition 3.2 , there are nonzero $\tilde{u}, \tilde{v} \in \mathbf{R}^{2}$ with $\tilde{u} \cdot \tilde{v}=0$ and $\psi$ being the coordinatewise median scheme with respect to $\{\tilde{u}, \tilde{v}\}$ with constant points $c^{1}, \ldots, c^{n+1}$. Hence by lemma $1.5, \varphi=B^{-1} \circ \psi \circ B$ is the coordinatewise median scheme with respect to $\{u, v\}$ with constant points $c^{1}, \ldots, c^{n+1}$, where $u=B^{-1} \tilde{u}$ and $v=B^{-1} \tilde{v}$. Furthermore, $\langle u, v\rangle=(B u) \cdot(B v)=$ $\left(B B^{-1} \tilde{u}\right) \cdot\left(B B^{-1} \tilde{v}\right)=\tilde{u} \cdot \tilde{v}=0$.

(if) Let nonzero $u, v \in \mathbf{R}^{2}$ with $\langle u, v\rangle=0$ and let $\varphi$ be the coordinatewise median scheme with respect to $\{u, v\}$ with constant points $c^{1}, \ldots, c^{n+1}$. Then it is evident that $\varphi$ is anonymous and componentwise continuous. Since $\langle u, v\rangle=0$, we have by lemma 2.2 that $u$ and $v$ are also $\|\cdot\|$-orthogonal $(\|\cdot\|$ being the norm induced by $(\cdot, \cdot)$. Hence by lemma $2.19, \varphi$ satisfies SP with respect to $\delta$.

Theorem 3.1 is a version of theorem 2.5 for the case that $\delta$ is induced by an inner product.

Theorem 3.1 Let the metric $\delta$ on $\mathbb{R}^{2}$ be induced by an inner product on $\mathbb{R}^{2}$. Then a voting scheme $\varphi:\left(\mathbb{R}^{2}\right)^{N} \rightarrow \mathbb{R}^{2}$ is anonymous, and Pareto optimal and strategy-proof with respect to $\delta$, if and only if there are nonzero $u, v \in \mathbb{R}^{2}$ with $\langle u, v\rangle=0$ and $\varphi$ being the coordinatewise median scheme with respect to $\{u, v\}$ without constant points.

Proof (only if) Let $\varphi:\left(\mathbb{R}^{2}\right)^{N} \rightarrow \mathbb{R}^{2}$ satisfy $\mathrm{AN}$, and PO and SP with respect to $\delta$. By $P O$ we can assume w.l.o.g. that $n>1$ (the dictatorial scheme is also a coordinatewise median if $n=1$ ). Then by corollary 3.2 , $\varphi$ is componentwise continuous. So by corollary $3.3, \varphi$ is a coordinatewise median scheme with respect to an orthogonal basis $\{u, v\}$ and $n+1$ constant points. Then by corollary $2.5 \varphi$ is a coordinatewise median scheme with respect to $\{u, v\}$ without constant points.

(if) Let nonzero $u, v \in \mathbb{R}^{2}$ with $\langle u, v\rangle=0$. Let $\varphi$ be the coordinatewise median scheme with respect to $\{u, v\}$ without constant points. Then the anonymity of $\varphi$ is evident. The Pareto optimality of $\varphi$ follows from lemma 
3.4. Furthermore, corollary 3.3 implies that $\varphi$ satisfies strategy-proofness with respect to $\delta$.

Corollary 3.4 guarantees the existence of voting schemes as described in theorem 3.1. This corollary is similar to theorem 2.6.

Corollary 3.4 Let $n$ be odd. Let the metric $\delta$ on $\mathbf{R}^{2}$ be induced by an inner product $\langle\cdot, \cdot\rangle$ on $\mathbb{R}^{2}$. Then there are voting schemes $\varphi:\left(\mathbb{R}^{2}\right)^{N} \rightarrow \mathbb{R}^{2}$ which are anonymous, and Pareto optimal and strategy-proof with respect to $\delta$.

Proof Let $u, v \in \mathbb{R}^{2} \backslash\{0\}$ be such that $\langle u, v\rangle=0$. Let $\varphi:\left(\mathbf{R}^{2}\right)^{N} \rightarrow \mathbf{R}^{2}$ be the coordinatewise median scheme with respect to $\{u, v\}$ without constant points. Then by theorem $3.1 \varphi$ satisfies $\mathrm{AN}$, and PO and SP with respect to $\delta$.

Since for all nonzero $u \in \mathbf{R}^{2}$ there is a nonzero $v \in \mathbb{R}^{2}$ such that $\langle u, v\rangle=0$, there are infinitely many voting schemes satisfying anonymity, Pareto optimality and strategy-proofness. This is not the case for arbitrary strictly convex norms, where the corresponding coordinate system may be unique.

The following corollary is a version of corollary 2.6 for inner product norms. We give here an alternative proof, using the theory developed in this chapter.

Corollary 3.5 Let $m \geq 2$, and let $n$ be even. Let the metric $\delta$ on $\mathbb{R}^{m}$ be induced by an inner product on $\mathbb{R}^{m}$. Then there is no scheme $\varphi:\left(\mathbb{R}^{m}\right)^{N} \rightarrow$ $\mathbf{R}^{m}$ which is anonymous, and Pareto optimal and strategy-proof with respect to $\delta$.

Proof Suppose $\varphi:\left(\mathbb{R}^{m}\right)^{N} \rightarrow \mathbb{R}^{m}$ satisfies AN, and $\mathrm{PO}$ and SP with respect to $\delta_{2}$. Let $u, v \in \mathbf{R}^{m}$ be linearly independent. Let $\psi$ be the restriction of $\varphi$ to $(\operatorname{span}\{u, v\})^{N}$. Then by lemma $3.3, \psi\left((\operatorname{span}\{u, v\})^{N}\right)=\operatorname{span}\{u, v\}$. It is easy to verify that $\psi$ also satisfies $\mathrm{AN}$, and $\mathrm{PO}$ and SP with respect to $\delta$. Hence, by theorem 3.1, $\psi$ is a coordinatewise median scheme without constant points. Since this scheme is only defined for an odd number of agents, this contradicts the assumption that $n$ is even.

Lemma 3.10 is only used to prove theorem 3.2 .

Lemma 3.10 Let $n \geq 3$. Let the metric $\delta$ on $\mathbb{R}^{3}$ be induced by the inner product $\langle\cdot, \cdot\rangle$ on $\mathbb{R}^{3}$. Let $\varphi:\left(\mathbf{R}^{3}\right)^{N} \rightarrow \mathbf{R}^{3}$ satisfy $A N$, and $P O$ and $S P$ with respect to $\delta$. Let disjoint nonempty $J, K, L \subset N$ with $J \cup K \cup L=N$. Let 
nonzero $u, v, w \in \mathbb{R}^{3}$ be such that $\langle u, v\rangle=\langle u, w\rangle=0$, and $\varphi\left(u^{J}, v^{K}, 0^{L}\right)=0$. Then $\varphi\left(u^{J}, w^{K}, 0^{L}\right)=0$.

Proof In view of theorem 3.1, for profiles $p$ with all components in a two dimensional subspace of $\mathbf{R}^{3}, \varphi$ must coincide with a coordinatewise median scheme without constant points in this subspace. Let nonzero $u, v, w \in \mathbb{R}^{3}$ with $\langle u, v\rangle=\langle u, w\rangle=0$ and $\varphi\left(u^{J}, v^{K}, 0^{L}\right)=0$. Suppose $\varphi\left(u^{J}, w^{K}, 0^{L}\right) \neq$ 0 . Then there are nonzero $\tilde{v}, \tilde{w} \in \mathbf{R}^{m}$ such that $\langle u, \tilde{v}\rangle=\langle u, \tilde{w}\rangle=0$, $\varphi\left(u^{J}, \tilde{v}^{K}, 0^{L}\right)=0, \varphi\left(u^{J}, \tilde{w}^{K}, 0^{L}\right) \neq 0$ and $\langle\tilde{v}, \tilde{w}\rangle>0$. Let $y=\varphi\left(u^{J}, \tilde{w}^{K}, 0^{L}\right)$. By lemma 3.3, $y \in \Delta(0, u, \tilde{w})$. Hence, there are $\lambda_{1}, \lambda_{2} \in[0,1]$ with $0<$ $\lambda_{1}+\lambda_{2} \leq 1$ and $y=\lambda_{1} u+\lambda_{2} \tilde{w}$. Since the restriction of $\varphi$ to $\operatorname{span}\{u, \tilde{v}\}$ coincides with a coordinatewise median scheme without constant points, $\varphi\left(u^{J},(\mu \tilde{v})^{K}, 0^{L}\right)=0$ for $\mu \in(0, \infty)$. So by SP, $\delta(\mu \tilde{v}, 0) \leq \delta(\mu \tilde{v}, y)$ for all $\mu \in(0, \infty)$. Hence, $\langle\mu \tilde{v}, \mu \tilde{v}\rangle \leq\left\langle\mu \tilde{v}-\lambda_{1} u-\lambda_{2} \tilde{w}, \mu \tilde{v}-\lambda_{1} u-\lambda_{2} \tilde{w}\right\rangle=$ $\langle\mu \tilde{v}, \mu \tilde{v}\rangle+\left\langle\lambda_{1} u, \lambda_{1} u\right\rangle+\left\langle\lambda_{2} \tilde{w}, \lambda_{2} \tilde{w}\right\rangle-2\left\langle\mu \tilde{v}, \lambda_{2} \tilde{w}\right\rangle$ for all $\mu \in(0, \infty)$. So $0<\lambda_{1}^{2}\langle u, u\rangle+\lambda_{2}^{2}\langle\tilde{w}, \tilde{w}\rangle-2 \lambda_{2} \mu\langle\tilde{v}, \tilde{w}\rangle$ for all $\mu \in(0, \infty)$. Hence, $\lambda_{2}=0$. Therefore, $y=\lambda_{1} u$ with $\lambda_{1} \in(0,1]$. So $\delta(\tilde{w}, y)=\sqrt{\left\langle\tilde{w}-\lambda_{1} u, \tilde{w}-\lambda_{1} u\right\rangle}=$ $\sqrt{\langle\tilde{w}, \tilde{w}\rangle+\lambda_{1}^{2}\langle u, u\rangle} \sqrt{\langle\tilde{w}, \tilde{w}\rangle}=\delta(\tilde{w}, 0)$. This contradicts SP. Consequently, $\varphi\left(u^{J}, w^{K}, 0^{L}\right)=0$.

For $m>2, n \geq 2$, anonymity, and Pareto optimality and strategy-proofness with respect to an inner product metric are inconsistent, as is stated by theorem 3.2. As is shown by example 2.13, this result does not hold for arbitrary strictly convex norms.

Theorem 3.2 Let $m \geq 3, n \geq 2$. Let the metric $\delta$ on $\mathbb{R}^{m}$ be induced by an inner product on $\mathbb{R}^{m}$. Then there is no scheme $\varphi:\left(\mathbb{R}^{m}\right)^{N} \rightarrow \mathbb{R}^{m}$ which is anonymous, and Pareto optimal and strategy-proof with respect to $\delta$.

Proof In view of corollary 3.5 we may assume w.l.o.g. that $n$ is odd. Hence, $n \geq 3$. We suppose that a scheme $\varphi$ with the three mentioned properties exists, and derive a contradiction. Let $k \in \mathbb{N}$ be such that $n=2 k+1$. Let disjoint $J, K, L \subset N$ with $|J|=|K|=k$ and $|L|=1$. Let $u, v \in \mathbb{R}^{m}$ be linearly independent. In view of theorem 3.1 , for profiles $p$ with all components in $(\text { aff }\{u, v\})^{N}, \varphi$ must be a coordinatewise median scheme without constant points, say with respect to $\{\tilde{u}, \tilde{v}\}$. W.l.o.g. $\{\tilde{u}, \tilde{v}\}$ is orthonormal. So we have that $\varphi\left(\tilde{u}^{J}, \tilde{v}^{K}, 0^{L}\right)=0$. Let $w \in \mathbf{R}^{m} \backslash\{0\}$ with $\langle\tilde{u}, w\rangle=\langle\tilde{v}, w\rangle=0$. By repeated application of lemma 3.10 we obtain successively:

$$
\varphi\left(\tilde{u}^{J}, v^{K}, 0^{L}\right)=0
$$


hence

$$
\varphi\left((\tilde{u}+\tilde{v})^{J}, v^{K}, 0^{L}\right)=0
$$

so

$$
\varphi\left((\tilde{u}+\tilde{v})^{J},(\tilde{u}-\tilde{v})^{K}, 0^{L}\right)=0 .
$$

On the other hand, by the definition of $\tilde{u}$ and $\tilde{v}, \varphi\left((\tilde{u}+\tilde{v})^{J},(\tilde{u}-\tilde{v})^{K}, 0^{L}\right)=\tilde{u}$. Since, $\tilde{u} \neq 0$, this gives the desired contradiction.

\subsection{Conclusion}

In this chapter we studied metrics which are induced by an inner product. The first main result is corollary 3.2 , which states that strategy-proofness and Pareto optimality with respect to such a metric imply componentwise continuity. Why it is important to establish componentwise continuity, will be explained in section 4.5 .

The second main result is theorem 3.1 , which characterizes all voting schemes which satisfy $\mathrm{AN}$, and $\mathrm{PO}$ and SP with respect to a norm which is induced by an inner product. This result is a special case of theorem 2.5 (combined with theorem 2.2). However, the proof of theorem 3.1 is significantly shorter, due to the use of a result of Kim and Roush (proposition 3.2).

It is an interesting question whether the Kim and Roush can be generalized to arbitrary strictly convex norms. If this is true as well as conjecture 3.2 , this would yield an alternative proof of theorem 2.5 .

In chapter 4 we replace Pareto optimality by unanimity. Again, we establish continuity, enabling us to characterize all voting schemes which satisfy unanimity, anonymity and strategy-proofness with respect to $\delta_{2}$. Since Pareto optimality implies unanimity, we also might use this result to prove theorem 3.1 (see also the remark at the end of section 4.4). The proof presented in this chapter however is more straightforward. 


\section{Chapter 4}

\section{Range Convexity and Euclidean Norms}

This chapter is based on Peters, Van der Stel and Storcken (1993b).

\subsection{Introduction}

In this chapter we consider schemes $\varphi:\left(\mathbf{R}^{m}\right)^{N} \rightarrow \mathbf{R}^{m}$ which satisfy strategyproofness with respect to the Euclidean metric $\delta_{2}$. It will be shown that for several cases range convexity and componentwise continuity are equivalent under the assumption of strategy-proofness. This leads to characterizations of certain classes of schemes.

Section 4.2 deals with the one person case. Using a result from convex analysis the equivalence of range convexity and continuity can easily be established. A characterization of all schemes satisfying strategy-proofness with respect to $\delta_{2}$ and continuity is given in theorem 4.1. Summarizing, section 4.2 is a generalization of section 2 in the paper of Kim and Roush (1984) for $m \geq 2$.

In section 4.3 we study schemes $\varphi:\left(\mathbf{R}^{m}\right)^{2} \rightarrow \mathbf{R}^{m}$ which satisfy strategyproofness with respect to $\delta_{2}$ and range convexity. The results will be used in section 4.4, where we consider $\varphi:\left(\mathbf{R}^{2}\right)^{N} \rightarrow \mathbf{R}^{2}$. In theorem 4.2 the equivalence of componentwise continuity and range convexity is established. This enables us to use a result of Kim and Roush (1984) in order to give some characterizations of scheme classes. Section 4.5 concludes.

Like in chapter 3 , the results in this chapter can be generalized to arbitrary metrics which are induced by an inner product, using the methodology 
developed in chapter 3. As this generalization is straightforward, we leave it to the interested reader.

\subsection{One person Euclidean location problems on $\mathbb{R}^{m}$}

In this section we study schemes $\varphi: \mathbf{R}^{m} \rightarrow \mathbf{R}^{m}$ which satisfy strategyproofness with respect to the Euclidean metric $\delta_{2}$. We will show that for such schemes range convexity is equivalent to continuity (theorem 4.1). The results of this section are generalizations of results of Kim and Roush (1984), section 2, who considered only the case $m=2$. Throughout this section we write $\varphi(x)$ instead of $\varphi\left(\left\langle x^{N}\right\rangle\right)$.

The following lemma extends theorem 12 in Kim and Roush (1984) to arbitrary $m$. Restricting Motzkin's theorem (Valentine, 1964, theorem 7.8; Motzkin, 1935) to the Euclidean case, we arrive at the following formulation:

Lemma 4.1 Let $C \subset \mathbf{R}^{m}$ be a closed set. Then the following assertions are equivalent:

(i) $C$ is convex and nonempty.

(ii) For all $y \in \mathbb{R}^{m}$ there is a unique $x \in C$ such that $\delta_{2}(x, y)$ is minimal.

The following two lemmas correspond more or less to proposition 11 in Kim and Roush (1984).

Lemma 4.2 Let $\delta$ be a metric on $\mathbf{R}^{m}$. Let $\varphi: \mathbf{R}^{m} \rightarrow \mathbf{R}^{m}$ satisfy strategyproofness with respect to $\delta$. Then the range $C$ of $\varphi$ is a closed (with respect to $\delta$ ) subset of $\mathbf{R}^{m}$.

Proof Let $x^{i} \in C(i \in \mathbf{N}), x \in \mathbb{R}^{m}$ with $\delta\left(x, x^{i}\right) \rightarrow 0$. Since $x^{i} \in C$, we can define $y^{i} \in \mathbf{R}^{m}$ such that $x^{i}=\varphi\left(y^{i}\right)$ for $i \in \mathbf{N}$. By SP, $\delta(x, \varphi(x)) \leq$ $\delta\left(x, \varphi\left(y^{i}\right)\right)$ for $i \in \mathbf{N}$. Hence, $\delta(x, \varphi(x))=0$. So $\varphi(x)=x$. Therefore, $x \in C$. Consequently, $C$ is closed.

Lemma 4.3 Let $\varphi: \mathbf{R}^{m} \rightarrow \mathbf{R}^{m}$ satisfy strategy-proofness with respect to $\delta_{2}$. Then $\varphi$ is continuous if and only if $\varphi$ is range convex.

Proof Let $C \subset \mathbf{R}^{m}$ denote the range of $\varphi$. Lemma 4.2 implies that $C$ is closed. 
(i) Let $\varphi$ be continuous. In order to prove that $\varphi$ is range convex, it is by lemma 4.1 sufficient to prove that for every $y \in \mathbf{R}^{m}$ there is a unique point of $C$ with minimal distance to $y$. So let $y \in \mathbb{R}^{m}$ and $u \in C$ with $\delta_{2}(y, u) \leq \delta_{2}(y, x)$ for all $x \in C$. Let $w=\varphi(y)$. Then by SP, $\delta_{2}(y, w)=\delta_{2}(y, u)$. Let $x^{i} \in[u, y)$ for $i \in \mathbb{N}$ with $x^{i} \rightarrow y$. By SP, $\delta_{2}\left(x^{i}, \varphi\left(x^{i}\right)\right) \leq \delta_{2}\left(x^{i}, u\right)$ and $\delta_{2}(y, u)=\delta_{2}(y, w) \leq \delta_{2}\left(y, \varphi\left(x^{i}\right)\right)$ for all $i \in \mathbf{N}$. This is only possible if $\varphi\left(x^{i}\right)=u$ for all $i \in \mathbb{N}$. Hence, $u=\varphi(y)$ by the continuity of $\varphi$. This shows that $u$ must be unique.

(ii) Let $\varphi$ be range convex. Let $x, x^{i} \in \mathbb{R}^{m}(i \in \mathbb{N})$ with $x^{i} \rightarrow x$ and $w=\varphi(x)$. By SP,

$$
\delta_{2}(x, w) \leq \delta_{2}(x, c) \text { for all } c \in C .
$$

Further,

$$
\begin{aligned}
\delta_{2}\left(x, \varphi\left(x^{i}\right)\right) & \leq \delta_{2}\left(x, x^{i}\right)+\delta_{2}\left(x^{i}, \varphi\left(x^{i}\right)\right) \\
& \leq \delta_{2}\left(x, x^{i}\right)+\delta_{2}\left(x^{i}, \varphi(x)\right) \\
& \leq 2 \delta_{2}\left(x, x^{i}\right)+\delta_{2}(x, w) .
\end{aligned}
$$

In view of this we assume w.l.o.g. that $\varphi\left(x^{i}\right)$ converges, say to $v$. In particular, we have

$$
\delta_{2}(x, v) \leq \delta_{2}(x, w) .
$$

By SP, $\delta_{2}(v, \varphi(v)) \leq \lim _{i \rightarrow \infty} \delta_{2}\left(v, \varphi\left(x^{i}\right)\right)=0$. Hence, $\varphi(v)=v$. So $v \in C$. By (4.1) and (4.2), $v$ and $w$ both minimize the (Euclidean) distance to $x$ over $C$. Therefore, by lemma 4.1,v=w. Since $x^{i}, x$ were arbitrary, this proves continuity of $\varphi$.

Theorem 4.1 is related to theorem 13 in Kim and Roush (1984).

Theorem 4.1 A scheme $\varphi: \mathbf{R}^{m} \rightarrow \mathbf{R}^{m}$ satisfies strategy-proofness with respect to $\delta_{2}$ and continuity if and only if there is a closed convex set $I \subset \mathbb{R}^{m}$ such that $\varphi(x)$ is the closest point of I to $x$ for all $x \in \mathbf{R}^{m}$.

Proof (i) Let $\varphi: \mathbf{R}^{m} \rightarrow \mathbf{R}^{m}$ satisfy strategy-proofness with respect to $\delta_{2}$ and continuity. Let $I=\varphi\left(\mathbf{R}^{m}\right)$. Then by lemma 4.2, $I$ is closed. Lemma 4.3 implies that $\varphi$ is range convex. Hence, $I$ is convex. So by SP, $\varphi(x)$ is a closest point of $I$ to $x$ for all $x \in \mathbf{R}^{m}$. By lemma 4.1, this point is unique.

(ii) Let $I \subset \mathbf{R}^{m}$ be closed and convex. Let $\varphi: \mathbf{R}^{m} \rightarrow \mathbf{R}^{m}$ be such that $\varphi(x)$ is a closest point of $I$ to $x$ for all $x \in \mathbf{R}^{m}$. Then $I$ is the range of $\varphi$. Hence, 
$\varphi$ is range convex. So lemma 4.3 implies that $\varphi$ is continuous. By definition $\varphi$ is strategy-proof with respect to $\delta_{2}$.

The results in this section cannot be generalized for arbitrary norms, as is shown by the following example.

Example 4.1 Let $\|\cdot\|_{\infty}$ denote the maximum norm on $\mathbf{R}^{2}$. Let $C=\{x \in$ $\left.\mathbf{R}^{2}: x_{2}=\frac{1}{2}\left|x_{1}\right|\right\}$. Let $\varphi: \mathbf{R}^{2} \rightarrow \mathbf{R}^{2}$ be defined by $\varphi(x)=\left(\begin{array}{c}x_{1} \\ \frac{1}{2}\left|x_{1}\right|\end{array}\right)$ for all $x \in \mathbf{R}^{2}$. Then it is clear that $C$ is the range of $\varphi$. For all $x \in \mathbf{R}^{2}, y \in C$ we have that

$$
\begin{aligned}
\|x-y\|_{\infty} & =\left|x_{1}-y_{1}\right|+\left|x_{2}-y_{2}\right| \\
& =\left|x_{1}-y_{1}\right|+\left|x_{2}-\frac{1}{2}\right| y_{1}|| \\
& =\left|x_{1}-y_{1}\right|+\left|x_{2}-\frac{1}{2}\right| x_{1}\left|+\frac{1}{2}\right| x_{1}\left|-\frac{1}{2}\right| y_{1}|| \\
& \geq\left|x_{1}-y_{1}\right|+\left|x_{2}-\frac{1}{2}\right| x_{1}||-\left|\frac{1}{2}\right| x_{1}\left|-\frac{1}{2}\right| y_{1}|| \\
& \geq\left|x_{2}-\frac{1}{2}\right| x_{1}||+\frac{1}{2}\left|x_{1}-y_{1}\right| \\
& >|| x-\left.\varphi(x)\right|_{\infty}
\end{aligned}
$$

except if $y=\varphi(x)$. Consequently, for all $x \in \mathbb{R}^{2}, \varphi(x)$ coincides with the unique point in $C$ for which the distance to $x$ is minimized. So $\varphi$ is strategyproof with respect to $\|\cdot\|_{\infty}$. By definition, $\varphi$ is also continuous. On the other hand, $C$ is not a convex set.

In the above example $\|\cdot\|_{\infty}$ can be replaced by the norm $\|\cdot\|$ defined by

$$
\|x-y\|=\|x-y\|_{\infty}+\frac{1}{4}\|x-y\|_{2}
$$

for all $x, y \in \mathbf{R}^{2}$. Note that this metric is strictly convex. Consequently, the results of this section cannot be generalized for arbitrary strictly convex norms.

\subsection{Two person Euclidean location problems}

In this section we consider voting schemes $\varphi:\left(\mathbf{R}^{m}\right)^{2} \rightarrow \mathbf{R}^{m}$ which satisfy range convexity and strategy-proofness with respect to the Euclidean metric $\delta_{2}$. The results of this section are mainly important for technical reasons, and 
will be used in section 4.4 where we focus on the case $m=2$. Throughout this section we denote $\varphi(x, y)$ instead of $\varphi\left(\left\langle x^{\{1\}}, y^{\{2\}}\right\rangle\right)$.

The lemmas in this section can best be explained by considering the socalled option set of agent 1 . The option set of agent 1 , given the reported point of agent 2, is the set of points that player 1 can enforce to be the compromise point, given the choice of agent 2 . Given that agent 2 chooses $y$, we will denote this set by $E(y)$. So, $E(y)=\left\{\varphi(x, y): x \in \mathbb{R}^{m}\right\}$. The expression 'option set' is due to Barberà and Peleg (1990). However, such sets were already used by Laffond (1980). Since we assume $\varphi$ to be strategyproof with respect to $\delta_{2}$, we also have confirmatoriness (see lemma 1.4), and thus $E(y)=\left\{x \in \mathbf{R}^{m}: x=\varphi(x, y)\right\}$.

As we will see in the next section, the convexity of all option sets is a necessary and sufficient condition for the continuity of $\varphi$ (cf. section 4.2).

The following two lemmas are used in the proof of lemma 4.6. Lemma 4.4 expresses a consequence of the nonconvexity of $E(y)$.

Lemma 4.4 Let $\varphi:\left(\mathbf{R}^{m}\right)^{2} \rightarrow \mathbf{R}^{m}$ satisfy strategy-proofness with respect to $\delta_{2}$. Let $v, w, y, z \in \mathbf{R}^{m}$ be such that $\varphi(v, y)=v, \varphi(w, y)=w, z \in(v, w)$ and $\varphi(z, y) \neq z$. Then there is a $\tilde{z} \in[z, v)$ with $\varphi(\tilde{z}, y) \neq \tilde{z}$ and $L(v, \tilde{z}, \varphi(\tilde{z}, y)) \leq$ $90^{\circ}$.

Proof Let $f:[z, v] \rightarrow \mathbb{R}$ be defined by $f(x)=\delta_{2}(x, \varphi(x, y))$ for all $x \in$ $|z, v|$. Lemma 2.16 implies that $f$ is continuous on $[v, z]$. Hence, we can take $\hat{z} \in[v, z]$ such that $f$ is maximized over $[v, z]$ at $\hat{z}$. Since $f(z)>0$, $f(\hat{z})>0$. In view of the continuity of $f$ we can take $\bar{z} \in(v, \hat{z})$ such that $f(\bar{z})<f(\hat{z})$ and $f(x)>0$ for all $x \in[\bar{z}, \hat{z}]$. Let $\tilde{z} \in[\bar{z}, \hat{z}]$ be such that $f(\tilde{z})$ is minimal on $[\bar{z}, \hat{z}]$. Then $\tilde{z} \neq \hat{z}$. Suppose $\angle(v, \tilde{z}, \varphi(\tilde{z}, y))>90^{\circ}$. Hence there is a $z^{*} \in[\hat{z}, \tilde{z})$ with $\delta_{2}\left(z^{*}, \varphi(\tilde{z}, y)\right)<\delta_{2}(\tilde{z}, \varphi(\tilde{z}, y))$. So by $\mathrm{SP}, f\left(z^{*}\right)=\delta_{2}\left(z^{*}, \varphi\left(z^{*}, y\right)\right) \leq \delta_{2}\left(z^{*}, \varphi(\tilde{z}, y)\right)<\delta_{2}(\tilde{z}, \varphi(\tilde{z}, y))=f(\tilde{z})$. This contradicts the minimality of $f(\tilde{z})$. Consequently, $\angle(v, \tilde{z}, \varphi(\tilde{z}, y)) \leq 90^{\circ}$.

Lemma 4.5 Let $\varphi:\left(\mathbf{R}^{m}\right)^{2} \rightarrow \mathbf{R}^{m}$ satisfy range convexity, and strategyproofness with respect to $\delta_{2}$. Let $v, w, x \in \mathbb{R}^{m}$ with $x \in[v, w], \varphi(v, v)=v$ and $\varphi(w, w)=w$. Then $\varphi(x, x)=x$.

Proof Since $v$ and $w$ are in the range of $\varphi$, we have by range convexity that $x$ is the range of $\varphi$. Hence there are $y, z \in \mathbb{R}^{m}$ with $\varphi(y, z)=x$. So by confirmatoriness of $\varphi$ (see lemma 1.4), $\varphi(x, x)=x$.

Lemma 4.6 asserts that the option set $E(v)$ is starshaped around $v$ if $v \in$ $E(v)$. 
Lemma 4.6 Let $\varphi:\left(\mathbb{R}^{m}\right)^{2} \rightarrow \mathbb{R}^{m}$ satisfy range convexity, and strategyproofness with respect to $\delta_{2}$. Let $v, w \in \mathbb{R}^{m}$ with $\varphi(v, v)=v$ and $\varphi(w, v)=$ $w$. Then $\varphi(x, v)=x$ for all $x \in[v, w]$.

Proof By confirmatoriness (see lemma 1.4), we have that $\varphi(w, w)=w$. Let $x \in[v, w]$. Suppose $\varphi(x, v) \neq x$. Then by lemma 4.4 we can take $\tilde{x} \in(v, w)$ such that $\varphi(\tilde{x}, v) \neq \tilde{x}$ and $\angle(w, \tilde{x}, \varphi(\tilde{x}, v)) \leq 90^{\circ}$. Hence $\delta_{2}(v, \tilde{x})<$ $\delta_{2}(v, \varphi(\tilde{x}, v))$. Also by lemma $4.5, \varphi(\tilde{x}, \tilde{x})=\tilde{x}$. So $\delta_{2}(v, \varphi(\tilde{x}, \tilde{x}))<\delta_{2}(v, \varphi(\tilde{x}$, $v)$ ). This contradicts SP.

If point $x$ is outside the option set $E(y)$, and $y$ is inside this set, the compromise point must be inside the smallest circle around $x$ and $y$.

Lemma 4.7 Let $\varphi:\left(\mathbb{R}^{m}\right)^{2} \rightarrow \mathbb{R}^{m}$ satisfy range convexity, and strategyproofness with respect to $\delta_{2}$. Let $x, y \in \mathbb{R}^{m}$ with $\varphi(y, y)=y$ and $\varphi(x, y) \notin$ $\{x, y\}$. Then $\angle(x, \varphi(x, y), y) \geq 90^{\circ}$.

Proof Let $u=\varphi(x, y)$. Suppose $\angle(x, u, y)<90^{\circ}$. Then we can take $\hat{x} \in(y, u)$ with $\delta_{2}(x, \hat{x})<\delta_{2}(x, u)$. Hence by SP,

$$
\varphi(\hat{x}, y) \neq \hat{x} .
$$

On the other hand, by confirmatoriness (see lemma 1.4), $\varphi(u, y)=u$. Hence, by lemma 4.6, $\varphi(\hat{x}, y)=\hat{x}$. This contradicts (4.3). Consequently, $\angle(x, \varphi(x, y), y) \geq 90^{\circ}$.

Lemma 4.8 extends positive association for the case that the compromise point is at one of the reported points.

Lemma 4.8 Let $\varphi:\left(\mathbf{R}^{m}\right)^{2} \rightarrow \mathbb{R}^{m}$ satisfy range convexity, and strategyproofness with respect to $\delta_{2}$. Let $x, y \in \mathbf{R}^{m}$ with $\varphi(x, y)=y$. Then $\varphi(z, y)=$ $y$ for all $z \in[y, x, \rightarrow)$.

Proof Let $z \in[y, x, \rightarrow)$. Let $w=\varphi(z, y)$. Suppose $w \neq y$. Then by confirmatoriness (see lemma 1.4), $z \neq y$, and by lemma 4.7, $\angle(z, y, w)<90^{\circ}$. So $x \neq y$ and $\angle(x, y, z)<90^{\circ}$. Hence, we can take $\hat{x} \in(y, w)$ such that

$$
\delta_{2}(x, \hat{x})<\delta_{2}(x, y)
$$

By confirmatoriness $\varphi(w, y)=w$ and $\varphi(y, y)=y$. Hence, by lemma 4.6, $\varphi(\hat{x}, y)=\hat{x}$. Because $\varphi(x, y)=y$, this implies by SP, $\delta(x, y) \leq \delta(x, \hat{x})$. This 
contradicts (4.4). Consequently, $\varphi(z, y)=y$.

If $x$ is in the convex hull of the option set $E(y)$, then the compromise point cannot be between $x$ and $y$ :

Lemma 4.9 Let $\varphi:\left(\mathbf{R}^{m}\right)^{2} \rightarrow \mathbf{R}^{m}$ salisfy range convexity, and strategyproofness with respect to $\delta_{2}$. Let $y, v, w \in \mathbb{R}^{m}$ with $\varphi(v, y)=v$ and $\varphi(w, y)=$ w. Let $x \in[v, w]$. Then $\varphi(x, y) \notin[y, x)$.

Proof Let $u=\varphi(x, y)$. Suppose $u \in[y, x)$. W.l.o.g. assume $\angle(v, x, u) \geq 90^{\circ}$ (otherwise interchange $v$ and $w$ ). Then we can take $z \in[y, x, \rightarrow) \backslash \mid y, x]$ such that $\delta_{2}(z, v)<\delta_{2}(z, u)$. By confirmatoriness (see lemma 1.4), $\varphi(x, u)=$ u. Hence, by lemma $4.8, \varphi(z, u)=u$. Let $a=\varphi(z, y)$. Then by SP, $\delta_{2}(y, a) \leq \delta_{2}(y, u)$ and $\delta_{2}(z, a) \leq \delta_{2}(z, v)<\delta_{2}(z, u)$. So, $\delta_{2}(y, a)+\delta_{2}(a, z)<$ $\delta_{2}(y, u)+\delta_{2}(z, u)=\delta_{2}(y, z)$. This contradicts the triangular inequality for metrics. Consequently, $\varphi(x, y) \notin[y, x)$.

The next two lemmas establish weak continuity properties of $\varphi$.

Lemma 4.10 Let $\varphi:\left(\mathbf{R}^{m}\right)^{2} \rightarrow \mathbf{R}^{m}$ satisfy range convexity, and strategyproofness with respect to $\delta_{2}$. Let $x, x^{i}, y \in \mathbf{R}^{m}(i \in \mathbf{N})$ with $x^{i} \rightarrow x$ and $\varphi(x, y)=y$. Then $\varphi\left(x^{i}, y\right) \rightarrow y$.

Proof By SP, $\delta_{2}\left(x, \varphi\left(x^{i}, y\right)\right) \leq \delta_{2}\left(x, x^{i}\right)+\delta_{2}\left(x^{i}, \varphi\left(x^{i}, y\right)\right) \leq \delta_{2}\left(x, x^{i}\right)+$ $\delta_{2}\left(x^{i}, \varphi(x, y)\right) \leq 2 \delta_{2}\left(x, x^{i}\right)+\delta_{2}(x, y)$ for all $i \in \mathbb{N}$. Hence, we can assume w.l.o.g. that $\varphi\left(x^{i}, y\right)$ converges, say to $w$, and

$$
\delta_{2}(x, w) \leq \delta_{2}(x, y) .
$$

Also, by SP, $\delta_{2}(w, \varphi(w, y)) \leq \lim _{i \rightarrow \infty} \delta_{2}\left(w, \varphi\left(x^{i}, y\right)\right)=0$. Hence,

$$
\varphi(w, y)=w .
$$

Suppose $w \neq y$, and let $\hat{x}=\frac{1}{2} y+\frac{1}{2} w$. Then by (4.5) and the strict convexity of $\delta_{2}$ (see lemma 3.1), we have by lemma 2.1 (iii) that $\delta_{2}(x, \hat{x})<\delta_{2}(x, y)$. Hence, by SP,

$$
\varphi(\hat{x}, y) \neq \hat{x}
$$

On the other hand, by confirmatoriness (see lemma 1.4), $\varphi(y, y)=y$. Hence by (4.6) and lemma 4.6, $\varphi(\hat{x}, y)=\hat{x}$, which contradicts (4.7). Consequently, $w=y$, so $\varphi\left(x^{i}, y\right) \rightarrow y$. 
Lemma 4.11 Let $\varphi:\left(\mathbb{R}^{m}\right)^{2} \rightarrow \mathbb{R}^{m}$ satisfy range convexity, and strategyproofness with respect to $\delta_{2}$. Let $x, x^{i}, w \in \mathbb{R}^{m}(i \in \mathbb{N})$ with $x^{i} \rightarrow x$ and $\varphi\left(x^{i}, w\right) \rightarrow w$. Then $\varphi(x, w)=w$.

Proof By strategy-proofness, $\delta_{2}(w, \varphi(w, w)) \leq \lim _{i \rightarrow \infty} \delta_{2}\left(w, \varphi\left(x^{i}, w\right)\right)=0$. Hence, $\varphi(w, w)=w$. Let $u=\varphi(x, w)$. Suppose $u \neq w$. Then by lemma 4.7, $\delta_{2}(x, u)<\delta_{2}(x, w)$. So for $i$ large enough, we have that $2 \delta_{2}\left(x, x^{i}\right)+$ $\delta_{2}\left(w, \varphi\left(x^{i}, w\right)\right)<\delta_{2}(x, w)-\delta_{2}(x, u)$, and so $\delta_{2}\left(x^{i}, u\right) \leq \delta_{2}\left(x^{i}, x\right)+\delta_{2}(x, u)<$ $-\delta_{2}\left(x^{i}, x\right)-\delta_{2}\left(w, \varphi\left(x^{i}, w\right)\right)+\delta_{2}(x, w) \leq \delta_{2}\left(x^{i}, \varphi\left(x^{i}, w\right)\right)$. This contradicts strategy-proofness.

The following, technical lemma will be used to establish continuity of $\varphi$ for the case $m=n=2$ in lemma 4.13 .

Lemma 4.12 Let $\varphi:\left(\mathbf{R}^{m}\right)^{2} \rightarrow \mathbf{R}^{m}$ satisfy range convexity, and strategyproofness with respect to $\delta_{2}$. If $\varphi$ is not componentwise continuous, there are $x, y, v, w \in \mathbf{R}^{m}$, with $v=\varphi(x, y), w=\varphi(w, y)=\varphi(x, w) \neq v, \delta_{2}(x, v)=$ $\delta_{2}(x, w)$ and $\delta_{2}(y, v)=\delta_{2}(y, w)$.

Proof Assume that $\varphi$ is not continuous in the first component. Then there are $x, x^{i}, y \in \mathbf{R}^{m}(i \in \mathbf{N})$ with $x^{i} \rightarrow x$ and

$$
\varphi\left(x^{i}, y\right) \nrightarrow \varphi(x, y) \text {. }
$$

Let

$$
v=\varphi(x, y)
$$

By strategy-proofness, we have that $\delta_{2}\left(x, \varphi\left(x^{i}, y\right)\right) \leq \delta_{2}\left(x, x^{i}\right)+\delta_{2}\left(x^{i}, \varphi\left(x^{i}\right.\right.$, $y)) \leq \delta_{2}\left(x, x^{i}\right)+\delta_{2}\left(x^{i}, \varphi(x, y)\right) \leq 2 \delta_{2}\left(x, x^{i}\right)+\delta_{2}(x, \varphi(x, y))$ for $i \in \mathbf{N}$. In view of this we assume w.l.o.g. that $\varphi\left(x^{i}, y\right)$ converges, say to $w$. By (4.8) and (4.9),

$$
v \neq w
$$

Hence,

$$
\begin{aligned}
\delta_{2}(x, w) & =\delta_{2}\left(x, \lim _{i \rightarrow \infty} \varphi\left(x^{i}, y\right)\right) \\
& =\lim _{i \rightarrow \infty} \delta_{2}\left(x, \varphi\left(x^{i}, y\right)\right) \\
& \leq \lim _{i \rightarrow \infty} 2 \delta_{2}\left(x, x^{i}\right)+\delta_{2}(x, \varphi(x, y))=\delta_{2}(x, v) .
\end{aligned}
$$

Also by $\operatorname{SP}, \delta_{2}(w, \varphi(w, y)) \leq \lim _{i \rightarrow \infty} \delta_{2}\left(w, \varphi\left(x^{i}, y\right)\right)=0$. Hence,

$$
\varphi(w, y)=w \text {. }
$$


Hence by SP, $\delta_{2}(x, v) \leq \delta_{2}(x, w)$. Together with (4.11) this implies that

$$
\delta_{2}(x, v)=\delta_{2}(x, w)
$$

By confirmatoriness (see lemma 1.4), $\varphi(x, v)=v$. So by lemma 4.10, $\varphi\left(x^{i}, v\right) \rightarrow v$. Hence by SP,

$$
\begin{aligned}
\delta_{2}(y, w) & =\delta_{2}\left(y, \lim _{i \rightarrow \infty} \varphi\left(x^{i}, y\right)\right) \\
& =\lim _{i \rightarrow \infty} \delta_{2}\left(y, \varphi\left(x^{i}, y\right)\right) \\
& \leq \lim _{i \rightarrow \infty} \delta_{2}\left(y, \varphi\left(x^{i}, v\right)\right) \\
& \leq \delta_{2}\left(y, \lim _{i \rightarrow \infty} \varphi\left(x^{i}, v\right)\right)=\delta_{2}(y, v) .
\end{aligned}
$$

Also by SP, $\delta_{2}\left(w, \varphi\left(x^{i}, w\right)\right) \leq \delta_{2}\left(w, \varphi\left(x^{i}, y\right)\right)$ for all $i \in \mathbf{N}$, hence $\varphi\left(x^{i}, w\right) \rightarrow$ $w$. So by lemma 4.11,

$$
\varphi(x, w)=w .
$$

Hence by SP, $\delta_{2}(y, v) \leq \delta_{2}(y, w)$. So by (4.14),

$$
\delta_{2}(y, v)=\delta_{2}(y, w) .
$$

Together with (4.9), (4.10), (4.12), (4.13), and (4.15) this gives the desired result. It is easy to see that we obtain the same result if $\varphi$ is not continuous in the second component.

\subsection{Two dimensional Euclidean location problems}

In this section we study voting schemes $\varphi:\left(\mathbf{R}^{2}\right)^{N} \rightarrow \mathbf{R}^{2}$ which satisfy strategy-proofness with respect to the Euclidean metric $\delta_{2}$. In lemmas 4.13 and 4.14 we consider the two-person case, where we can use the results of section 4.3. Next we can use these lemmas for the more general $n$-person case $(n \geq 2)$. Theorem 4.2 states that for such schemes range convexity is equivalent to componentwise convexity. This enables us to use results obtained by Kim and Roush (1984), to give an alternative characterization of the class of coordinatewise median schemes with $n+1$ constant points. By replacing range convexity by stronger axioms like surjectivity or unanimity we obtain characterizations of subclasses of this scheme set.

The following lemma is the analagon of lemma 4.3 , which is on the one person location problem. 
Lemma 4.13 Let $\varphi:\left(\mathbf{R}^{2}\right)^{2} \rightarrow \mathbf{R}^{2}$ satisfy range convexity, and strategyproofness with respect to $\delta_{2}$. Then $\varphi$ is componenturise continuous.

Proof Suppose $\varphi$ is not componentwise continuous. Then lemma 4.12 implies that there are $x, y, v, w \in \mathbb{R}^{m}$, with $v=\varphi(x, y), w=\varphi(w, y)=$ $\varphi(x, w) \neq v$,

$$
\delta_{2}(x, v)=\delta_{2}(x, w)
$$

and

$$
\delta_{2}(y, v)=\delta_{2}(y, w)
$$

Let $d=\frac{1}{2} v+\frac{1}{2} w$. (See figure 4.1.) Let $u=\varphi(d, y)$. By (4.16),

$$
\delta_{2}(x, d)<\delta_{2}(x, v)
$$

So by SP, $u \neq d$. Hence by lemma 4.9, $u \notin[y, d]$. By confirmatoriness we have, that $\varphi(v, v)=v$ and $\varphi(w, w)=w$. So lemma 4.5 implies that $\varphi(d, d)=d$. Therefore, by lemma $4.7, \angle(y, u, d) \geq 90^{\circ}$. Hence,

$$
\delta_{2}(y, u)<\delta_{2}(y, d)
$$

W.l.o.g. assume that $\angle(w, d, u)<90^{\circ}$ (otherwise interchange the roles of $v$ and $w)$. Then we can take $z \in[u, d, \rightarrow) \backslash|u, d|$ with $\angle(w, v, z)=90^{\circ}$. Let $a=\varphi(z, y)$. Then by SP, $\delta_{2}(z, a) \leq \delta_{2}(z, v)$. Hence,

$$
[y, a] \cap(\leftarrow, v, w, \rightarrow) \neq 0 \text {. }
$$

By confirmatoriness (see lemma 1.4), $\varphi(d, u)=u$. Hence, lemma $4.8 \mathrm{im}$ plies that $\varphi(z, u)=u$. So by SP and $(4.19), \delta_{2}(y, a) \leq \delta_{2}(y, u)<\delta_{2}(y, d)$. Therefore, by (4.18), $[y, a] \cap(\leftarrow, v, w, \rightarrow)=0$. This contradicts $(4.20)$. Consequently, $\varphi$ is componentwise continuous.

We conjecture that lemma $\mathbf{4 . 1 3}$ is valid for arbitrary dimension $m$.

Conjecture 4.1 Let $\varphi:\left(\mathbb{R}^{m}\right)^{2} \rightarrow \mathbb{R}^{m}$ satisfy range convexity, and strategyproofness with respect to $\delta_{2}$. Then $\varphi$ is componentuise continuous.

It is also interesting to investigate if this conjecture holds for other strictly convex norms.

Lemma 4.14 establishes the convexity of all option sets.

Lemma 4.14 Let $\varphi:\left(\mathbb{R}^{2}\right)^{2} \rightarrow \mathbb{R}^{2}$ satisfy range convexity, and strategyproofness with respect to $\delta_{2}$. Let $v, w, y \in \mathbf{R}^{2}$ with $\varphi(v, y)=v$ and $\varphi(w, y)=$ $w$. Then $\varphi(x, y)=x$ for all $x \in[v, w]$. 


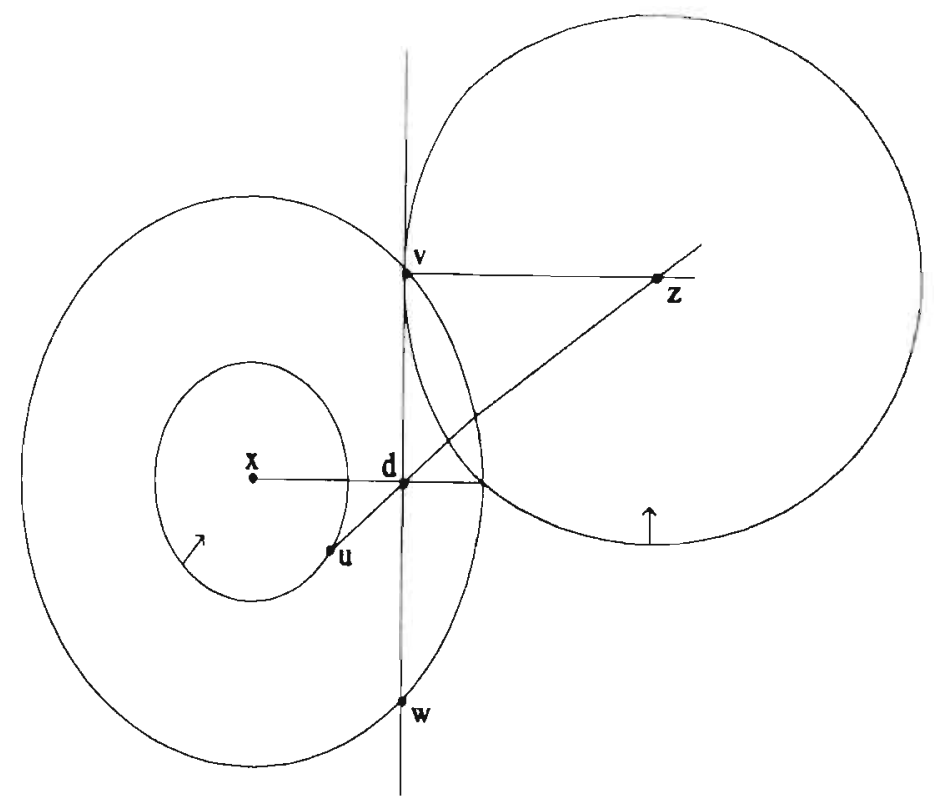

Figure 4.1: Proof of lemma 4.15

Proof Let $\psi: \mathbf{R}^{2} \rightarrow \mathbf{R}^{2}$ be defined by $\psi(x)=\varphi(x, y)$ for $x \in \mathbb{R}^{2}$. Since $\varphi$ is strategy-proof with respect to $\delta_{2}, \psi$ is strategy-proof with respect to $\delta_{2}$ (cf. lemma 1.2). By lemma 4.13, $\varphi$ is componentwise continuous. So $\psi$ is continuous. Hence by lemma $4.3, \psi$ is range convex. Let $x \in[v, w]$. Then by range convexity, $x$ is in the range of $\psi$. So $x=\psi(u)$ for some $u \in \mathbb{R}^{2}$. Therefore, by confirmatoriness (see lemma 1.4), $\psi(x)=x$. Consequently, $\varphi(x, y)=x$.

Lemma 4.15 extends this result to the case with more than 2 agents.

Lemma 4.15 Let $\varphi:\left(\mathbb{R}^{2}\right)^{N} \rightarrow \mathbb{R}^{2}$ satisfy range convexity and strategyproofness with respect to $\delta_{2}$. Let $k \in N$. Let $S=\{k\}, p \in\left(\mathbb{R}^{2}\right)^{N \backslash S}$. Let $v, w \in \mathbf{R}^{2}$ with $\varphi\left(\left\langle v^{S}, p\right\rangle\right)=v$ and $\varphi\left(\left\langle w^{S}, p\right\rangle\right)=w$. Then $\varphi\left(\left\langle x^{S}, p\right\rangle\right)=x$ for all $x \in[v, w]$.

Proof W.l.o.g. assume $k=1$. Let $S_{j}=\{1, \ldots, j\}$ for $j \in N$. Let $p^{j} \in$ $\left(\mathbf{R}^{2}\right)^{N \backslash S_{j}}$ be such that $p^{j}=\left.p\right|_{N \backslash S_{j}}$ for $j \in N$. Let $i$ be the smallest element of $N$ such that for all $\tilde{v}, \tilde{w}, \tilde{x} \in \mathbf{R}^{m}$ with $\varphi\left(\left\langle\tilde{v}^{S_{i}}, p^{i}\right\rangle\right)=\tilde{v}, \varphi\left(\left\langle\tilde{w}^{S_{i}}, p^{i}\right\rangle\right)=\tilde{w}$ and $\tilde{x} \in[\tilde{v}, \tilde{w}]$ we have that $\varphi\left(\left\langle\tilde{x}^{S_{i}}, p^{i}\right\rangle\right)=\tilde{x}$. Since the range convexity of $\varphi$ and confirmatoriness (see lemma 1.4) imply that the statement holds for 
$i=n$, a smallest $i \in N$ for which it holds certainly exists.

Suppose $i>1$. Let $\psi:\left(\mathbb{R}^{2}\right)^{2} \rightarrow \mathbf{R}^{2}$ be defined by $\psi(x, y) \equiv \varphi\left(\left\langle x^{S_{i-1}}, y^{\{i\}}\right.\right.$, $\left.\left.p^{i}\right\rangle\right)$. Then by lemma $1.2 \psi$ satisfies strategy-proofness with respect to $\delta_{2}$. By the definition of $i, \psi$ also satisfies range convexity. So lemma $4.14 \mathrm{im}-$ plies that for all $\tilde{v}, \tilde{w}, \tilde{x} \in \mathbb{R}^{2}$ with $\psi(\tilde{v}, p(i))=\tilde{v}, \psi(\tilde{w}, p(i))=\tilde{w}$ and $\tilde{x} \in[\tilde{v}, \tilde{w}]$ we have that $\psi(\tilde{x}, p(i))=\tilde{x}$. Hence, for all $\tilde{v}, \tilde{w}, \tilde{x} \in \mathbf{R}^{2}$ with $\varphi\left(\left\langle\tilde{v}^{S_{i-1}}, p^{i-1}\right\rangle\right)=\tilde{v}, \varphi\left(\left\langle\tilde{w}^{S_{i-1}}, p^{i-1}\right\rangle\right)=\tilde{w}$ and $\tilde{x} \in[\tilde{v}, \tilde{w} \mid$ we have that $\varphi\left(\left\langle\tilde{x}^{S_{i-1}}, p^{i-1}\right\rangle\right)=\tilde{x}$. This contradicts the definition of $i$.

Consequently, $i=1$. So for all $\tilde{v}, \tilde{w}, \tilde{x} \in \mathbb{R}^{m}$ with $\varphi\left(\left\langle\tilde{v}^{S}, p\right\rangle\right)=\tilde{v}$, $\varphi\left(\left\langle\tilde{w}^{S}, p\right\rangle\right)=\tilde{w}$ and $\tilde{x} \in[\tilde{v}, \tilde{w}]$ we have that $\varphi\left(\left\langle\tilde{x}^{S}, p\right\rangle\right)=\tilde{x}$. Hence, $\varphi\left(\left\langle x^{S}, p\right\rangle\right)=x$ for all $x \in[v, w]$.

Theorem 4.2 extends theorem 4.1 to the case with more than 2 agents, under the assumption that $m=2$. It also extends lemma 4.13 .

Theorem 4.2 Let $\varphi:\left(\mathbf{R}^{2}\right)^{N} \rightarrow \mathbb{R}^{2}$ satisfy strategy-proofness with respect to $\delta_{2}$. Then $\varphi$ is range convex if, and only if, $\varphi$ is componentwise continuous.

Proof (i) Let $\varphi$ be range convex. Let $k \in N$ be arbitrary. Let $p \in\left(\mathbf{R}^{2}\right)^{N \backslash\{k\}}$ be arbitrary. Let $\psi: \mathbf{R}^{2} \rightarrow \mathbb{R}^{2}$ be defined by $\psi(x)=\varphi\left(\left\langle x^{\{k\}}, p\right\rangle\right)$ for all $x \in \mathbf{R}^{2}$. Then by lemma $4.15, \psi$ is range convex. Hence by lemma $4.3, \psi$ is continuous. Since $p$ was chosen arbitrarily, this implies that $\varphi$ is continuous in the $k$-th component. Since $k$ was chosen arbitrarily, this implies that $\varphi$ is componentwise continuous.

(ii) Let $\varphi$ be componentwise continuous. Let $\psi: \mathbf{R}^{2} \rightarrow \mathbf{R}^{2}$ be defined by $\psi(x)=\varphi\left(\left\langle x^{N}\right\rangle\right)$ for all $x \in \mathbf{R}^{2}$. Then $\psi$ is continuous. Lemma 1.2 implies that $\psi$ also satisfies strategy-proofness with respect to $\delta_{2}$. Hence by lemma $4.3, \psi$ is range convex. Confirmatoriness (see lemma 1.4) implies that the range of $\varphi$ coincides with the range of $\psi$. Consequently, $\varphi$ is range convex.

If conjecture 4.1 is true, this theorem can be generalized to arbitrary dimension $m$. So we have:

Conjecture 4.2 Let $\varphi:\left(\mathbb{R}^{m}\right)^{N} \rightarrow \mathbb{R}^{m}$ satisfy strategy-proofness with respect to $\delta_{2}$. Then $\varphi$ is range convex if, and only if, $\varphi$ is componentwise continuous.

If this conjecture is true, it implies proposition 3.1, which also establishes componentwise continuity for arbitrary $m$ and $n$, using the much stronger 
Pareto optimality condition instead of range convexity. Using a result of Kim and Roush (1984), quoted by our proposition 3.2 , we obtain:

Theorem 4.3 Let $n \geq 2$. Then a voting scheme $\varphi:\left(\mathbb{R}^{2}\right)^{N} \rightarrow \mathbb{R}^{2}$ is anonymous, range convex, and strategy-proof with respect to $\delta_{2}$ if, and only if, there are nonzero $u, v \in \mathbf{R}^{2}$ and $c^{1}, \ldots, c^{n+1} \in \overline{\mathbf{R}}^{2}$ with $u \cdot v=0$ and $\varphi$ being the coordinatewise median scheme with respect to $\{u, v\}$ with constant points $c^{1}, \ldots, c^{n+1}$.

Proof Theorem 4.2 implies that if $\varphi$ is strategy-proof with respect to $\delta_{2}$, then $\varphi$ is range convex if and only if $\varphi$ is componentwise continuous. The desired result now follows from proposition 3.2 .

So in the result of Kim and Roush (1984) (see proposition 3.2), continuity can be replaced by range convexity. Surjectivity implies range convexity. This leads to:

Corollary 4.1 Let $n \geq 2$. Then a voting scheme $\varphi:\left(\mathbb{R}^{2}\right)^{N} \rightarrow \mathbf{R}^{2}$ is anonymous, surjective, and strategy-proof with respect to $\delta_{2}$ if, and only if, there are nonzero $u, v \in \mathbb{R}^{2}$ and $c^{1}, \ldots, c^{n-1} \in \mathbb{R}^{2}$ with $u \cdot v=0$ and $\varphi$ being the coordinatewise median scheme with respect to $\{u, v\}$ with $n-1$ constant points $c^{1}, \ldots, c^{n-1}$.

Proof (i) Let $\varphi:\left(\mathbb{R}^{2}\right)^{N} \rightarrow \mathbb{R}^{2}$ satisfy AN, SURJ and SP with respect to $\delta_{2}$. By surjectivity the range of $\varphi$ coincides with $\mathbf{R}^{2}$. So $\varphi$ is range convex. Hence theorem 4.3 implies that there are nonzero $u, v \in \mathbf{R}^{2}$ with $u \cdot v=0$ and $\varphi$ being the coordinatewise median scheme with respect to $\{u, v\}$ with constant points $\tilde{c}^{1}, \ldots, \tilde{c}^{n+1}$. By the surjectivity of $\varphi, \varphi$ must coincide with the coordinatewise median scheme with respect to $\{u, v\}$ with constant points $c^{1}, \ldots, c^{n-1}$.

(ii) Let nonzero $u, v \in \mathbf{R}^{2}$ with $u \cdot v=0$ and $\varphi$ be the coordinatewise median scheme with respect to $\{u, v\}$ with $n-1$ constant points. Then $\varphi\left(\left\langle x^{N}\right\rangle\right)=x$ for all $x \in \mathbb{R}^{2}$. Hence $\varphi$ is surjective. The anonymity of $\varphi$ is evident. The strategy-proofness of $\varphi$ follows from lemma 2.17 (note that orthogonality and $\|\cdot\|_{2}$-orthogonality are equivalent by lemma 2.2$)$.

Under the assumption of strategy-proofness, unanimity and surjectivity are equivalent (see corollary 1.1). So we have: 
Corollary 4.2 Let $n \geq 2$. Then a voting scheme $\varphi:\left(\mathbb{R}^{2}\right)^{N} \rightarrow \mathbb{R}^{2}$ is anonymous, unanimous and strategy-proof with respect to $\delta_{2}$, if and only if there are nonzero $u, v \in \mathbf{R}^{2}$ with $u \cdot v=0$, and $\varphi$ being the coordinatewise median scheme with respect to $\{u, v\}$ with $n-1$ constant points $c^{1}, \ldots, c^{n-1}$.

Proof Corollary 1.1 states that for strategy-proof schemes unanimity and surjectivity are equivalent. The desired result now follows from corollary 4.1 .

Since (weak) Pareto optimality implies unanimity (see lemma 1.3), theorem 3.1 follows easily from this corollary.

\subsection{Conclusion}

In this chapter we have studied voting schemes $\varphi:\left(\mathbb{R}^{m}\right)^{N} \rightarrow \mathbf{R}^{m}$ which satisfy range convexity, and strategy-proofness with respect to the Euclidean metric $\delta_{2}$. All the results we found, are also valid with respect to all metrics which are induced by an inner product. The proof of this statement is straightforward (using the methodology developed in chapter 3 ).

In section 4.2 we consider the $m$-dimensional one person location problem. We show that the results $\mathrm{Kim}$ and Roush (1984) presented in their section 2 are also valid for higher dimensions.

An important result of this chapter is theorem 4.2. This theorem establishes for $m=2$ the equivalence of range convexity and componentwise continuity, under the assumption of strategy-proofness with respect to $\delta_{2}$. Continuity can be used to derive other results ( $c$ f. the role of lemma 2.36 in chapter 2). There are also several authors who use componentwise continuity as a basic assumption (cf. Laffond (1980), Moulin (1984), Kim and Roush (1984), Bordes, Laffond and Le Breton (1990)). In our view, it is worthwhile to derive continuity from axioms which have a more direct economic or decision-theoretic significance, such as Pareto optimality, strategy-proofness or unanimity. Furthermore, by combining these results with a proof of continuity on the basis of other axioms, new results are easily obtained. Anyway, it seems to be useful to establish continuity, it might prove its worth in future research. 


\section{Chapter 5}

\section{Uncompromisingness}

This chapter is based on Peters, Van der Stel and Storcken (1991).

\subsection{Introduction}

In this chapter we study strategy-proofness with respect to a class of preferences on $A$, denoted by $P^{*}$. Briefly $P^{*}$ is the class of all preferences which have at least one globally best point, and are nonincreasing if we move away from a best point in any coordinate. Due to this structure, $P^{*}$ satisfies the tops-onlyness condition. In the larger part of this chapter we assume $A$ to be open.

The main object of this chapter is to characterize all voting schemes which are strategy-proof with respect to $P^{*}$. It will be demonstrated that a scheme is strategy-proof with respect to $P^{*}$ if and only if it is uncompromising. A scheme is uncompromising if moving an individually reported point around in one and the same closed orthant with respect to the compromise point, does not influence that compromise point. This condition is therefore a generalization of the uncompromisingness condition in Border and Jordan (1983).

It is proved that a scheme is uncompromising if and only if it is a standard coordinatewise veto scheme, as defined in section 1.3. Thus, a scheme is strategy-proof with respect to $P^{*}$ if, and only if, it is a coordinatewise veto scheme. We show that not all preferences of $P^{*}$ are needed but, essentially, a subset of preferences which makes the scheme being determined coordinatewise, i.e., consistent in the terminology of Rubinstein and Fishburn (1986).

The main result in Moulin (1980) - his Proposition 3 - shows that the 
coordinatewise veto schemes are exactly all schemes that are strategy-proof with respect to the class of single-peaked preferences on the whole real line. This chapter, in particular corollary 5.1, extends this result to arbitrary nonempty sets of alternatives in arbitrary dimensions, for a specific class of (essentially single-peaked) preferences, the class $P^{\circ}$ mentioned above.

Also the results of Border and Jordan (1983) are extended, in more than one direction. First, the family of all strategy-proof schemes is characterized, no other condition on the schemes being required. Border and Jordan's main result, theorem 1 , is derived as a corollary (corollary 5.2 ) for a more general set of alternatives. A byproduct of our approach is that the proofs seem to become more elementary and straightforward. For instance, it is demonstrated that strategy-proofness with respect to the class of separable and quadratic preferences as in theorem 1 of Border and Jordan (1983) implies strategy-proofness with respect to a set of preferences that depend only on one dimension. This shortcut greatly facilitates the proof, shows what essentially drives Border and Jordan's result, and consequently makes it easily extendable to other domains of preferences.

Second, the results in this chapter are derived under only mild restrictions on the set of alternatives, or no restrictions - except nonemptiness at all. Therefore, consequences of these results for various sets of alternatives as occurring in economic or political models can easily be established. An example is theorem 5.4, where the set of alternatives is a grid and all schemes are characterized which are (strictly) strategy-proof with respect to preferences determined by the sumnorm, a natural distance measure for a grid if we think of it as of an idealized road system. A related result is obtained by Barberà et al. (1990): See section 5.5 below.

There is a stream of other related literature which will not be discussed in detail here. We mention Satterthwaite and Sonnenschein (1981), Chichilnisky and Heal (1983), Moreno and Walker (1990), Barberà and Peleg (1990), Hurwicz and Walker (1990), Moreno (1991), Zhou (1991), Sprumont (1991). In this literature a common element is that, although the domains of preferences are restricted, they contain enough preferences to make a strategy-proof scheme being determined coordinatewise with respect to a system of coordinates fixed by the preferences.

In chapters 2, 3 and 4, as well as in Kim and Roush (1984) and Bordes et al. (1990), Euclidean preferences or, more generally, strictly convex norms are considered, which do not fix a specific system of coordinates. This makes it much harder to derive characterizations of strategy-proof schemes.

The organization of this chapter is as follows. Section 5.2 introduces the 
class of admissable preferences on a subset $A$ of $\mathbf{R}^{m}$, and a property named uncompromisingness. In section 5.3 we focus again on standard coordinatewise veto schemes, as discussed in section 1.3.

Properties of these schemes, possibly depending on more specific information about the set of alternatives, are derived. It is shown that such a scheme has a certain core property: no coalition (subset of individuals) can improve the outcome in all coordinates simultaneously. This result establishes a link between the model in this paper and cooperative game theory, whereas strategy-proofness is a noncooperative game-theoretic property. In section 5.4 the main results concerning strategy-proofness, uncompromisingness and confirmatoriness as cited above, are derived. In section $5.5 \mathrm{a}$ few special cases are considered where the strategy-proofness condition can be relaxed due to additional properties of the set of alternatives. Two of these cases, the 'grid' theorem and the extension of theorem 1 in Border and Jordan (1983), were discussed above. Section 5.6 concludes.

\subsection{The class of preferences $P^{*}$ and uncompro- misingness}

In this section we consider the set $P^{*}$ on a set $A \subset \mathbf{R}^{m}$. This class is defined with respect to the standard coordinate system in $\mathbf{R}^{m}$. Thereafter we discuss a property called uncompromisingness, which is also defined with respect to this standard coordinate system.

For all $x, y \in A$ the box between $x$ and $y$ is defined as

$$
\operatorname{box}(x, y):=\left\{z \in A: \forall t \in M\left[\min \left\{x_{t}, y_{t}\right\} \leq z_{t} \leq \max \left\{x_{t}, y_{t}\right\}\right]\right\}
$$

We define $P^{*}$ to be the class of all preferences such that:

(R1) $\operatorname{best}(R) \neq \emptyset$.

(R2) For all $x \in \operatorname{best}(R), y \in A, v \in \operatorname{box}(x, y):(v, y) \in R$.

Thus a preference $R \in P^{*}$ has best elements, and preference is (weakly) decreasing when moving away, in any coordinate(s), from such a best element. Observe that, if $R$ is transitive, then $x, y \in \operatorname{best}(R)$ implies $z \in \operatorname{best}(R)$ for all $z \in \operatorname{box}(x, y)$. In particular, this entails that best $(R)$ is a convex set if $A$ is convex and $R$ transitive. Throughout this chapter attention will be restricted to preferences in $P^{*}$. Note that $P^{*}$ is nonstandard (see section $1.2)$. 
When restricted to the one-dimensional case, $P^{*}$ is slightly larger than the class of single-peaked preferences considered in Moulin (1980), since best $(R)$ may contain more than one element if $R \in P^{*}$ and preferences in $P^{*}$ need not to be strictly decreasing when moving away from an ideal point. For $p \in[1, \infty)$ we define $\rho^{p}$ to be the class of preferences induced by the norm $\|\cdot\|_{p}$ (see section 2.3 ), i.e.,

$$
p^{p}=\left\{R \subset A \times A: \exists x \in A:\left[(y, z) \in R \Leftrightarrow\|x-y\|_{p} \leq\|x-z\|_{p}\right\} .\right.
$$

It is easy to verify that $p^{p} \subset p^{*}$ for all $p \in[1, \infty)$. Similarly, we define $p^{\infty}$ to be the class of preferences induced by $\|\cdot\|_{\infty}$. So $P^{\infty} \subset P^{*}$. Other subclasses of $P^{*}$ are the classes $P_{\alpha}$ with $\alpha \in(0, \infty)$ (see section 5.4) and the classes $Q^{k}$ with $k \in(0, \infty)$ (see subsection 5.5.2).

The two main scheme properties in this chapter are strategy-proofness and uncompromisingness. A scheme $\varphi$ is uncompromising if for all $t \in M$ and all $p, q \in A^{N}$ satisfying for all $i \in N$ :

$$
\begin{aligned}
& p(i)_{t}<\varphi(p)_{t} \Rightarrow q(i)_{t} \leq \varphi(p)_{t} \\
& p(i)_{t}>\varphi(p)_{t} \Rightarrow q(i)_{t} \geq \varphi(p)_{t}
\end{aligned}
$$

we have: $\varphi(p)_{t}=\varphi(q)_{t}$.

Uncompromisingness implies that the scheme can be determined coordinatewise, and hence implies the consistency condition of Rubinstein and Fishburn (1986). It was first introduced by Border and Jordan (1983) for the case $m=1$. In section 5.4 (lemma 5.9) it will be shown that, under the assumption that $A$ is an open subset of $\mathbf{R}^{m}$, strategy-proofness with respect to $P^{*}$ is equivalent to uncompromisingness.

\subsection{Coordinatewise veto schemes}

Standard coordinatewise veto schemes, as defined in section 1.3 , play an important role in this chapter. In the next section it will be proved that every scheme which is strategy-proof with respect to $P^{*}$ belongs to this class - actually, less will be needed for this to be true. The converse, that every coordinatewise veto scheme is strategy-proof with respect to $P^{*}$, is also true. Although this fact could be derived from Moulin (1980) or Border and Jordan (1983) by modifications of the corresponding results, for completeness' sake the present section gives a self-contained argument. Furthermore, these schemes will be related to effectivity functions (see, e.g., Abdou and Keiding (1991)) and to the concept of the core. 
Standard coordinatewise veto(pre)schemes can be defined as follows (cf. the general definition in section 1.3):

A map $\gamma: 2^{N} \rightarrow \overline{\mathbf{R}}^{m}$ is an assignment of disagreements if, for all $t \in M$ $\gamma(\emptyset)_{t}>-\infty, \gamma(N)_{t}<\infty$, and $\gamma(S)_{t} \geq \gamma(T)_{t}$ for all $t \in M$ and all $S \subset T \subset$ $N$. To such an assignment of disagreements $\gamma$ a prescheme $\varphi_{\gamma}$ is associated, as follows:

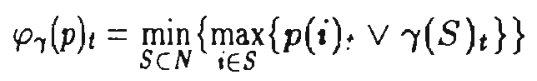

for all $p \in A^{N}$ and $t \in M . \varphi_{\gamma}$ is called a standard coordinatewise veto prescheme. Dependent on $A$ and $\gamma, \varphi_{\gamma}$ may be a scheme. In that case, $\gamma$ is a standard coordinatewise veto scheme.

Throughout this chapter, we assume $\varphi_{\gamma}$ to be a standard coordinatewise veto scheme.

Suppose a standard coordinatewise veto scheme $\varphi_{\gamma}$ is used. Let $S \subset N$ be a coalition. Clearly, $S$ can enforce $\varphi_{\gamma}(p)_{t}$ to be at most $\gamma(S)_{t}$ for every $t \in M$, by reporting points $p(i)(i \in S)$ with $p(i)_{t} \leq \gamma(S)_{t}$, provided there are such points in $A$. Under the same proviso, $S$ can enforce $\varphi_{\gamma}(p)_{t}$ to be at least $\gamma(N \backslash S)_{t}$, by reporting points $p(i)(i \in S)$ with $p(i)_{t} \geq \gamma(N \backslash S)_{t}$. More generally, for every $t \in M, S$ can enforce

$$
\varphi_{\gamma}(p)_{t} \in\left[\gamma\left(N \backslash S_{1}\right)_{t}, \gamma\left(S_{2}\right)_{t}\right]
$$

for all coalitions $S_{1}, S_{2} \subset S$ with $\gamma\left(N \backslash S_{1}\right)_{t} \leq \gamma\left(S_{2}\right)_{t}$. This more general formulation is based on the condition $S \subset T \Rightarrow \gamma(S)_{t} \geq \gamma(T)_{t}$ for an assignment of disagreements $\gamma$.

The foregoing considerations suggest the definition of an effectivity function $E(S \mid t)$ for a coalition $S$ on coordinate $t \in M$. Let

$$
\pi_{t}(X):=\left\{x_{t} \in \mathbf{R}: x \in X\right\}
$$

denote the projection of a set $X \subset \mathbb{R}^{m}$ on the $t$-th coordinate $t \in M$. Define $E(S \mid t)$ as the collection of those subsets $B \subset \pi_{t}(A)$ for which there exist $S_{1}$, $S_{2} \subset S$ satisfying (E1) and (E2), where:

(E1) $\left[\gamma\left(N \backslash S_{1}\right)_{t}, \gamma\left(S_{2}\right)_{t}\right] \cap \pi_{t}(A) \neq \emptyset$

(E2) $\left[\gamma\left(N \backslash S_{1}\right)_{t}, \gamma\left(S_{2}\right)_{t}\right] \cap \pi_{t}(A) \subset B$.

Here, for all $\alpha, \beta \in \overline{\mathbf{R}}, \mid \alpha, \beta]:=\{x \in \mathbf{R}: \alpha \leq x \leq \beta\}$.

It should be noted that, for a coalition $S$, enforcing a set $B \in E(S \mid t)$ may prevent the same coalition from enforcing a set $B^{\prime} \in E\left(S \mid t^{\prime}\right)$ in a different 
coordinate. This depends on the shape of $A$ and the location of the disagreement points. In other words, the effectivity functions should be interpreted as describing what each coalition can enforce coordinatewise, and not in all coordinates simultaneously. However, if, for example, the rectangular subset determined by the disagreement points is completely contained in $A$, then each coalition can enforce the subsets described by the effectivity function simultaneously in all coordinates.

For a profile of individual preferences $r \in\left(P^{*}\right)^{N}$ and an assignment of disagreements $\gamma$, the "core" of $r$ given $\gamma$ will be defined as the set of those alternatives of $A$ such that in every coordinate there is no coalition that can enforce better alternatives. What a coalition is able to enforce is determined by the effectivity functions defined above. It will be shown that this core always contains the alternative assigned by the coordinatewise veto. scheme associated with $\gamma$ to a profile in $A^{N}$ where each individual $i$ reports a best element of his preference $r(i)$.

In order to formalize these remarks, we first define, for a preference $R \in \mathcal{P}^{*}$, its projection on the $t$-th coordinate by

$$
\pi_{t}(R):=\left\{(\alpha, \beta) \in \mathbb{R}^{2}: \exists x, y \in A\left[x_{t}=\alpha, y_{t}=\beta, x=_{M \backslash\{t\}} y,(x, y) \in R\right]\right\} .
$$

Observe that this definition admits cases like $(\alpha, \beta) \in \pi_{t}(R)$ whereas $(x, y) \notin$ $R$ for certain $x, y \in A$ with $x_{t}=\alpha, y_{t}=\beta, x={ }_{M \backslash\{t\}} y$. Such occurrences are excluded when the preference $R$ is separable (i.e., for all $x, y, a, b \in A$ and all $t \in M$ such that $x={ }_{M \backslash\{t\}} y, a={ }_{M \backslash\{t\}} b, a_{t}=x_{t}, b_{t}=y_{t}$ and $(x, y) \in R$, we have: $(a, b) \in R)$. For what follows, however, we do not need separability of the preferences.

Let now $r \in\left(P^{*}\right)^{N}$ be a profile of preferences. Denote the set of $t$ coordinates which are better than $\alpha \in \mathbb{R}$ at $r$ for all $i \in S \subset N$ by:

$\operatorname{better}_{t}(\alpha, r, S):=\left\{\beta \in \pi_{t}(A): \forall i \in S\left[(\beta, \alpha) \in \pi_{t}(r(i)) \&(\alpha, \beta) \notin \pi_{t}(r(i))\right]\right\}$

Let $\gamma$ be an assignment of disagreements and $E(S \mid t)(S \subset N, t \in M)$ the corresponding effectivity functions. We define the core of $r$ given $\gamma$ by

$$
\operatorname{core}_{\gamma}(r):=\left\{x \in A: \forall t \in M, S \subset N\left[\operatorname{better}_{t}\left(x_{t}, r, S\right) \notin E(S \mid t)\right]\right\} .
$$

Thus, the core of $r$ given $\gamma$ consists exactly of those alternatives in $A$ such that, coordinatewise, no coalition can enforce its set of better outcomes given the effectivity function $E$. Elements of core $(r)$ are stable in this sense.

Before we can identify scheme alternatives $\varphi_{\gamma}(p)$ as core elements, we first give a further characterization of coordinatewise veto schemes. 
Lemma 5.1 Let $\gamma$ be an assignment of disagreements, $p \in A^{N}$, and $t \in M$. For all $\alpha \in \mathbf{R}$ let

$$
S_{\alpha}:=\left\{i \in N: p(i)_{t} \leq \alpha\right\} \text { and } T_{\alpha}:=\left\{i \in N \mid p(i)_{t}<\alpha\right\} .
$$

Then:

(i) If $\alpha=\varphi_{\gamma}(p)_{t}$, then $\gamma\left(S_{\alpha}\right)_{t} \leq \alpha \leq \gamma\left(T_{\alpha}\right)_{t}$.

(ii) If $\alpha \in \mathbf{R}$ with $\gamma\left(S_{\alpha}\right)_{t} \leq \alpha \leq \gamma\left(T_{\alpha}\right)_{t}$, then $\varphi_{\gamma}(p)_{t}=\alpha$.

(iii) $\varphi_{\gamma}(p)_{t}=\max _{S \subset N}\left\{\min _{i \in S}\left\{p(i)_{t} \wedge \gamma(N \backslash S)_{t}\right\}\right\}$.

Proof Let $S \subset N$ such that $\max _{i \in S}\left\{p(i)_{t} \vee \gamma(S)_{t}\right\}=\varphi_{\gamma}(p)_{t}$.

(i) Let $\alpha:=\varphi_{\gamma}(p)_{t}$. Then $\max _{i \in T_{\alpha}}\left\{p(i)_{t}\right\}<\alpha$. Therefore, by definition of $\varphi_{\gamma}, \gamma\left(T_{\alpha}\right)_{t} \geq \alpha$. Since $\max _{i \in S}\left\{p(i)_{t}\right\} \leq \alpha, S \subset S_{\alpha}$. Therefore, $\gamma\left(S_{\alpha}\right)_{t} \leq$ $\gamma(S)_{t} \leq \alpha$.

(ii) Let $\alpha \in \mathbf{R}$ with $\gamma\left(S_{\alpha}\right)_{t} \leq \alpha \leq \gamma\left(T_{\alpha}\right)_{t}$. Let $\beta:=\varphi_{\gamma}(p)_{t}$. Then $\beta=$ $\max _{i \in S}\left\{p(i)_{t} \vee \gamma(S)_{t}\right\} \leq \max _{i \in S_{\alpha}}\left\{p(i)_{t} \vee \gamma\left(S_{\alpha}\right)_{t}\right\} \leq \alpha$. Suppose $\beta<\alpha$. Then $\gamma(S)_{t}<\alpha \leq \gamma\left(T_{\alpha}\right)_{t}$, so $S \not \subset T_{\alpha}$. Therefore, $\max _{i \in S}\left\{p(i)_{t}\right\} \geq \alpha$, so $\beta \geq \alpha$, which is a contradiction. Thus, $\alpha=\beta$.

(iii) Let $\alpha:=\varphi_{\gamma}(p)_{t}$. By (i), $\min _{i \in N \backslash T_{\alpha}}\left\{p(i)_{t} \wedge \gamma\left(T_{\alpha}\right)_{t}\right\} \geq \alpha$. Therefore,

$$
\max _{T \subset N}\left\{\min _{i \in T}\left\{p(i)_{t} \wedge \gamma(N \backslash T)_{t}\right\}\right\} \geq \alpha .
$$

Suppose $T \subset N$ is such that $\min _{i \in T}\left\{p(i)_{t} \wedge \gamma(N \backslash T)_{t}\right\}>\alpha$. Then $S_{\alpha} \subset N \backslash T$, hence $\gamma(N \backslash T)_{t} \leq \gamma\left(S_{\alpha}\right)_{t} \leq \alpha$, where the last inequality follows from (i). This is clearly impossible. Therefore,

$$
\max _{T \subset N}\left\{\min _{i \in T}\left\{p(i)_{t} \wedge \gamma(N \backslash T)_{t}\right\}\right\} \leq \alpha .
$$

The desired conclusion follows from combining (5.1) and (5.2).

We are now in a position to prove the following theorem.

Theorem 5.1 Let $\gamma$ be an assignment of disagreements, and let $r \in\left(P^{*}\right)^{N}$. Then:

$$
\left\{\varphi_{\gamma}(p): \forall i \in N[p(i) \in \operatorname{best}(r(i))]\right\} \cap A \subset \operatorname{core}_{\gamma}(r) .
$$

Proof Take $p(i) \in \operatorname{best}(r(i))$ for every $i \in N$. Let $a:=\varphi_{\gamma}(p)$, assume $a \in A$. Suppose there exist $t \in M$ and $V \subset N$ with $B:=\operatorname{better}_{t}\left(a_{t}, r, V\right) \in E(V \mid t)$. Then there are $V_{1}, V_{2} \subset V$ with $\emptyset \neq\left[\gamma\left(N \backslash V_{1}\right)_{t}, \gamma\left(V_{2}\right)_{t}\right] \cap \pi_{t}(A) \subset B$. Let $\alpha:=a_{t}$. By lemma 5.1 (i), $\gamma\left(S_{\alpha}\right)_{t} \leq \alpha \leq \gamma\left(T_{\alpha}\right)_{t}$, notations as there. 
Suppose $p(i)_{t}<\alpha$ for some $i \in V$. Suppose there exists a $\beta \in B$ with $\beta \geq \alpha$. By definition of $B,(\alpha, \beta) \notin \pi_{t}(r(i))$ and $(\beta, \alpha) \in \pi_{t}(r(i))$. So there are $x, y \in A$ with $x_{t}=\alpha, y_{t}=\beta, x={ }_{M \backslash\{t\}} y,(y, x) \in r(i)$. Since $(\alpha, \beta) \notin \pi_{t}(r(i))$, we have $(x, y) \notin r(i)$. This however, combined with $p(i) \in$ best $(r(i))$ and $x \in$ box $(p(i), y)$, contradicts condition (R2) for the preference $r(i) \in p^{*}$. Therefore, $p(i)_{t}<\alpha$ for some $i \in V$ implies $B \subset(-\infty, \alpha)$. Similarly, it can be shown that $p(i)_{t}>\alpha$ for some $i \in V$ implies $B \subset(\alpha, \infty)$, and $p(i)_{t}=\alpha$ for some $i \in V$ implies $B=\emptyset$. We conclude that either $p(i)_{t}<\alpha$ for all $i \in V$ or $p(i)_{t}>\alpha$ for all $i \in V$.

If $p(i)_{t}<\alpha$ for all $i \in V$, then $V \subset T_{\alpha}$, and $\gamma\left(V_{2}\right)_{t}<\alpha$ since $B \subset$ $(-\infty, \alpha)$. Therefore, $\gamma\left(T_{\alpha}\right)_{t} \leq \gamma(V)_{t} \leq \gamma\left(V_{2}\right)_{t}<\alpha$, a contradiction. If $p(i)_{t}>\alpha$ for all $i \in V$, then $V \subset N \backslash S_{\alpha}$ and $\gamma\left(N \backslash V_{1}\right)_{t}>\alpha$ since $B \subset(\alpha, \infty)$. Therefore, $\gamma\left(S_{\alpha}\right)_{t} \geq \gamma\left(N \backslash V_{1}\right)_{t}>\alpha$, a contradiction. We conclude that $t$ and $V$ as above cannot exist, hence $\varphi_{\gamma}(p) \in \operatorname{core}_{\gamma}(r)$.

Next, it will be shown that every coordinatewise veto scheme is strategyproof with respect to $P^{*}$.

Lemma 5.2 Let $\gamma$ be an assignment of disagreements, such that $\varphi_{\gamma}$ is a standard coordinatewise veto scheme. Then $\varphi_{\gamma}$ is strategy-proof with respect to $P^{*}$.

Proof Let $i \in N, R \in p^{*}, p, q \in A^{N}$ with $p={ }_{N \backslash\{i\}} q$ and $p(i) \in \operatorname{best}(R)$. Denote $a:=\varphi_{\gamma}(p)$ and $b:=\varphi_{\gamma}(q)$. It will be proved that $a \in$ box $(p(i), b)$, from which the desired conclusion follows in view of (R2).

Let $t \in M$. Without loss of generality assume $p(i)_{t}<a_{t}$. If $q(i)_{t} \geq p(i)_{t}$, then for all $S \subset N$ :

$$
\max _{j \in S} q(j)_{t} \geq \max _{j \in S} p(j)_{t}
$$

hence

$$
\max _{j \in S} q(j)_{t} \vee \gamma(S)_{t} \geq \max _{j \in S} p(j)_{t} \vee \gamma(S)_{t} .
$$

So in this case, $b_{t} \geq a_{t}>p(i)_{t}$.

If $q(i)_{t}<p(i)_{t}$ then

$$
\gamma\left(\left\{j \in N: p(j)_{t} \leq a_{t}\right\}\right)=\gamma\left(\left\{j \in N: q(j)_{t} \leq a_{t}\right\}\right)
$$

and

$$
\gamma\left(\left\{j \in N: p(j)_{t}<a_{t}\right\}\right)=\gamma\left(\left\{j \in N q(j)_{t}<a_{t}\right\}\right),
$$

therefore, by lemma 5.1 (ii), $b_{t}=a_{t}$. 
Observe that also theorem 5.1 entails a kind of strategy-proofness condition on a scheme $\varphi_{\gamma}$. Namely, as a consequence of this theorem, there is no coordinate $t \in M$ and no coalition $S \subset N$ such that $S$ can enforce the set of $t$-coordinates which are better than $\varphi_{\gamma}(p)_{t}$ for all members of $S$. In other words, for every set of $t$-coordinates enforceable by $S$, i.e., every set $B \in E(S \mid t)$, there is always an individual $i \in S$ and a number $\alpha \in B$ such that $i$ does not strictly prefer $\alpha$ to $\varphi_{\gamma}(p)_{t}$. This is a weak form of coalitional strategy-proofness.

In section 5.2 the property of uncompromisingness was introduced. Standard coordinatewise veto schemes satisfy this property.

Lemma 5.3 Let $\gamma$ be an assignment of disagreements, and let $\varphi_{\gamma}$ be a standard coordinatewise veto scheme. Then $\varphi_{\gamma}$ is uncompromising.

Proof Straightforward from the definition of $\varphi_{\gamma}$.

The last part of this section is devoted to a study of special well-known instances of coordinatewise veto schemes, and to Pareto optimality conditions for (coordinatewise veto) schemes.

Example 5.1 Let $t \in M, i \in N$, and $\gamma$ an assignment of disagreements such that, for all $S \subset N$ :

$$
\gamma(S)_{t}:=\left\{\begin{array}{rll}
\infty & \text { if } & i \notin S \\
-\infty & \text { if } & i \in S
\end{array}\right.
$$

Obviously, $\varphi_{\gamma}(p)_{t}=p(i)_{t}$ for every $p \in A^{N}: i$ is a dictator on the $t$-th coordinate. (Cf. the dictatorial scheme, where there is an $i \in N$ which is dictator on every coordinate.)

Example 5.2 Let $\varphi: A^{N} \rightarrow A$ be the coordinatewise median scheme (as defined in section 1.3) with respect to the standard unit basis with $n+1$ constant points $c^{i}$. By definition we may assume w.l.o.g. that $c_{t}^{1} \leq c_{t}^{2} \leq$ $\cdots \leq c_{t}^{n+1}$ for all $t \in M$. So we have:

$$
\varphi(p)_{t}=\operatorname{med}\left(p(1)_{t}, \ldots, p(n)_{t}, c_{t}^{1}, \ldots, c_{t}^{n+1}\right)
$$

for all $t \in M, p \in A^{N}$.

For $k=1, n+1$ and every $S \subset N$ with $|S|=k$ define $\gamma(S):=c^{k+1}$. Then $\gamma$ is an assignment of disagreements. Let $\varphi_{\gamma}$ be the associated standard coordinatewise veto scheme. We claim that $\varphi_{\gamma}=\varphi$. In order to prove this 
claim, let $t \in M$ and $\alpha:=\varphi_{\gamma}(p)_{t}$, where $p \in A^{N}$. By lemma 5.1 (i), with notations as there, we have $\gamma\left(S_{\alpha}\right)_{t} \leq \alpha \leq \gamma\left(T_{\alpha}\right)_{t}$. So

$$
\begin{aligned}
& \left|\left\{i \in\{1, \ldots, n+1\}: c_{t}^{i} \leq \alpha\right\}\right|+\left|\left\{i \in N: p(i)_{t} \leq \alpha\right\}\right| \geq \\
& n-\left|S_{A}\right|+\left|S_{A}\right|=n+1 \text {, }
\end{aligned}
$$

and

$$
\begin{aligned}
& \left|\left\{i \in\{1, \ldots, n+1\}: c_{t}^{i} \geq \alpha\right\}\right|+\left|\left\{i \in N: p(i)_{t} \geq \alpha\right\}\right| \geq \\
& \left|T_{\alpha}\right|+1+n-\left|T_{\alpha}\right|=n+1 .
\end{aligned}
$$

It follows that $\operatorname{med}\left(p(1)_{t}, \ldots, p(n)_{t}, c_{t}^{1}, \ldots, c_{t}^{n+1}\right)=\alpha$. Hence, $\varphi(p)_{t}=$ $\varphi_{\gamma}(p)_{t}$.

Lemma 5.4 Let $\gamma$ be an assignment of disagreements with $\left\{x \in \mathbf{R}^{m}\right.$ : $\gamma(N) \leq x \leq \gamma(\theta)\} \subset A$. Then the following three statements are equivalent:

(i) $\varphi_{\gamma}$ is anonymous.

(ii) $\gamma(S)=\gamma(T)$ for all $S, T \subset N$ with $|S|=|T|$.

(iii) $\varphi_{\gamma}$ is a coordinatewise median scheme.

Proof Let $S \subset N$ with $0<|S|<n, k \in S, l \notin S, T=(S \backslash\{k\}) \cup\{l\}$. Suppose $\gamma(S) \neq \gamma(T)$, w.l.o.g. $\gamma(S)_{t}<\gamma(T)_{t}$ for some $t \in M$. Take a profile $p \in A^{N}$ with $p(i)_{t}=\gamma(S)_{t}$ if $i \in S$ and $p(i)_{t}=\gamma(T)_{t}$ if $i \notin S$. Let $\sigma$ be the permutation of $N$ defined by $\sigma(k)=l, \sigma(l)=k, \sigma(i)=i$ for $i \neq k, l$. Then $\varphi_{\gamma}(p)_{t}=\gamma(S)_{t}$ and $\varphi_{\gamma}(p \circ \sigma)_{t}=\gamma(T)_{t}$, so $\varphi$ cannot be anonymous. From this, the implication (i) $\Rightarrow$ (ii) follows. For the implication (ii) $\Rightarrow$ (iii), observe that $\varphi$ is a coordinatewise median scheme with respect to the standard basis with $n+1$ constant points $c^{i}$, with $c^{i}=\gamma(\{1, \ldots, i-1\})$ for $i=1, \ldots, n+1$. The implication (iii) $\Rightarrow$ (i) follows immediately from the definition of $\varphi$.

Lemma 5.5 Let $\gamma$ be an assignment of disagreements. Then $\varphi_{\gamma}$ is coordinatewise Pareto optimal if and only if $A \subset\left\{x \in \mathbb{R}^{m}: \gamma(N) \leq x \leq \gamma(\theta)\right\}$.

Proof Straightforward, left to the reader. 


\subsection{Characterizing coordinatewise veto schemes}

In this section it will be proved that every uncompromising scheme must be a standard coordinatewise veto scheme. As a corollary of this characterization it will follow that also every scheme which is strategy-proof with respect to $P^{*}$, must be a coordinatewise veto scheme.

Lemma 5.6 Let $\varphi: A^{N} \rightarrow A$ be an uncompromising scheme. Let $t \in M$ and $S \subset N$, and $a, b, c, x, y, z \in A$ with $\varphi\left(a^{S}, b^{N \backslash S}\right)=c$ and $\varphi\left(x^{S}, y^{N \backslash S}\right)=$ z. Then:

(i) If $c_{t} \leq a_{t}<b_{t}$ and $x_{t}<y_{t} \leq z_{t}$ then $z_{t} \leq c_{t}$.

(ii) If $x_{t} \leq a_{t}<c_{t}<b_{t} \leq y_{t}$ then $z_{t}=c_{t}$.

(iii) If $c_{t} \leq a_{t}<b_{t}, x_{t}<y_{t} \leq z_{t}$, and $\varphi\left(x^{S}, b^{N \backslash S}\right)=d$, then $x_{t}<d_{t}<b_{t}$.

Let $l_{t}:=\inf \left(\pi_{t}(A)\right)$ and $u_{t}:=\sup \left(\pi_{t}(A)\right)$. Then:

(iv) $\lim _{x, y: x_{t} \downarrow l_{t}, y_{t} \uparrow u_{t}} \varphi\left(x^{S}, y^{N \backslash S}\right)_{t}$ exists.

Proof (i) Let $c_{t} \leq a_{t}<b_{t}$ and $x_{t}<y_{t} \leq z_{t}$, and suppose $z_{t}>c_{t}$. Then by uncompromisingness, $\varphi\left(c^{S}, z^{N \backslash S}\right)_{\mathfrak{t}}=c_{\mathfrak{t}}$. Similarly, $\varphi\left(x^{S}, z^{N-S}\right)=z$, hence $\varphi\left(c^{S}, z^{N \backslash S}\right)_{t}=z_{t}$. So we have a contradiction.

(ii) Let $x_{t} \leq a_{t}<c_{t}<b_{t} \leq y_{t}$. Suppose $z_{t} \leq c_{t}$. By uncompromisingness, $\varphi\left(z^{S}, y^{N \backslash S}\right)=z$ and $\varphi\left(a^{S}, c^{N \backslash S}\right)=c$. So $a_{t}<c_{t} \leq c_{t}$ and $z_{t} \leq z_{t}<y_{t}$. So by (i), $c_{t} \leq z_{t}$. Hence $z_{t}=c_{t}$. The assumption $z_{t} \geq c_{t}$ implies $z_{t}=c_{t}$ in an analogous way.

(iii) Under the conditions stated, we have by (i): $x_{t}<y_{t} \leq z_{t} \leq c_{t} \leq$ $a_{t}<b_{t}$. The inequality $d_{t} \leq x_{t}$ would, by uncompromisingness, imply $z_{t}=\varphi\left(x^{S}, y^{N \backslash S}\right)_{t}=d_{t} \leq x_{t}$, a contradiction. Hence, $x_{t}<d_{t}$. Analogously, one derives $d_{t}<b_{t}$.

(iv) If $a_{t}=b_{t}=: \alpha$ for all $a, b \in A$, then obviously the limit exists and is equal to $\alpha$. If $a_{t}<\varphi\left(a^{S}, b^{N \backslash S}\right)_{t}<b_{t}$ for some $a, b \in A$, then we are done by (ii). In the remaining case, $l_{t}<u_{t}$ and $a_{t}<\varphi\left(a^{S}, b^{N \backslash S}\right)_{t}<b_{t}$ for no $a, b \in A$. Then, by (iii), either $\varphi\left(a^{S}, b^{N \backslash S}\right)_{t} \leq a_{t}$ for al] $a, b \in A$ with $a_{t}<b_{t}$, or $\varphi\left(a^{S}, b^{N \backslash S}\right)_{t} \geq b_{t}$ for all $a, b \in A$ with $a_{t}<b_{t}$. Thus, the limit exists and equals either $l_{t}$ or $u_{t}$.

Let $\varphi: A^{N} \rightarrow A$ be an uncompromising scheme. For every $S \subset N$ and $t \in M$ define

$$
\gamma(S)_{t}:=\lim _{x, y: x_{t} \downarrow_{t}, v_{i} \dagger u_{t}} \varphi\left(x^{S}, y^{N \backslash S}\right) .
$$

Then $\gamma: 2^{N} \rightarrow(\bar{R})^{m}$ is well-defined in view of lemma 5.6 (iv). 
Lemma 5.7 Let $\varphi: A^{N} \rightarrow A$ be an uncompromising scheme, and let $\gamma$ be defined as in (5.9). Then:

(i) Let $\varphi\left(a^{S}, b^{N \backslash S}\right)=a$. Then $a_{t}<b_{t} \Rightarrow \gamma(S)_{t} \leq a_{t}$ and $a_{t}>b_{t} \Rightarrow$ $\gamma(N \backslash S)_{t} \geq a_{t}$, for all $t \in M$.

(ii) $\gamma$ is an assignment of disagreements.

(iii) For all $S \subset N$ and $x, y \in A$, if $\varphi\left(x^{S}, y^{N \backslash S}\right)=x$, then $\varphi_{\gamma}\left(x^{S}, y^{N \backslash S}\right)=$ $x$.

Proof (i) Let $\varphi\left(a^{S}, b^{N \backslash S}\right)=a, t \in M$, and $a_{t}<b_{t}$. Suppose $\gamma(S)_{t}>a_{t}$. Then, by (5.3), there are $x, y \in A$ with $x_{t} \leq a_{t}<z_{t} \leq \gamma(S)_{t}, a_{t}<y_{t}$, $\varphi\left(x^{S}, y^{N \backslash S}\right)=z$. So by uncompromisingness, $\varphi\left(a^{S}, z^{N \backslash S}\right)_{t}=z_{t}$. Also by uncompromisingness, $\varphi\left(a^{S}, z^{N \backslash S}\right)_{t}=a_{t}$. So we have a contradiction. The proof of the second implication is analogous.

(ii) By uncompromisingness, there is an $a \in A$ with $\varphi\left(a^{N}, b^{\emptyset}\right)=a$ for all $b \in A$. Let $t \in M$. If $a_{t}=\max \left(\pi_{t}(A)\right)$ then $\gamma(N)_{t}<\infty$. Otherwise, let $a_{t}<b_{t}$, then (i) implies $\gamma(N)_{t} \leq a_{t}<\infty$. One similarly shows $\gamma(\emptyset)_{t}>-\infty$. Let $S \subset T \subset N$. Suppose $\gamma(S)_{t}<\gamma(T)_{t}$. Then there are $x, y \in A$ with $\varphi\left(x^{S}, y^{N \backslash S}\right)=a, \varphi\left(x^{T}, y^{N \backslash T}\right)=b$, and $a_{t}<b_{t}$. Further, w.l.o.g., $x_{t}<b_{t}$ and $a_{t}<y_{t}$. Uncompromisingness implies $\varphi\left(a^{S}, y^{N \backslash S}\right)=a, \varphi\left(x^{T}, b^{N \backslash T}\right)=b$, $\varphi\left(x^{S}, b^{N \backslash S}\right)=b$. Then lemma 5.6 (i) implies $a_{t} \geq b_{t}$, a contradiction.

(iii) Let $S \subset N$ and $x, y \in A$ with $\varphi\left(x^{S}, y^{N \backslash S}\right)=x$. Let $t \in M$. If $x_{t}<y_{t}$, then, by (i), $\gamma(S)_{t} \leq x_{t}$. By definition this implies $\varphi_{\gamma}\left(x^{S}, y^{N \backslash S}\right)_{t}=x_{t}$. If $x_{t}>y_{t}$, then $\gamma(N \backslash S)_{t} \geq x_{t}$ by (i). Now $\varphi_{\gamma}\left(x^{S}, y^{N \backslash S}\right)_{t}=x_{t}$ follows by lemma 5.1 (iii). Finally, suppose $x_{t}=y_{t}$, so $\varphi\left(x^{N}, y^{\theta}\right)_{t}=x_{t}$. By (5.3) and uncompromisingness, this implies $\gamma(N)_{t} \leq x_{t} \leq \gamma(\emptyset)_{t}$. Therefore, $\varphi_{\gamma}\left(x^{S}, y^{N \backslash S}\right)_{t}=x_{t}$.

Lemma 5.8 Let $\varphi: A^{N} \rightarrow A$ be an uncompromising scheme and $\gamma$ an assignment of disagreements, such that for all $S \subset N$ and $x, y \in A$, if $\varphi\left(x^{S}, y^{N \backslash S}\right)=x$, then $\varphi_{\gamma}\left(x^{S}, y^{N \backslash S}\right)=x$. Then $\varphi=\varphi_{\gamma}$.

Proof Take $t \in M$ and $p \in A^{N}$. It is sufficient to prove that $\varphi(p)_{t}=$ $\varphi_{\gamma}(p)_{t}$. Let $a=\varphi(p), b=\varphi_{\gamma}(p)$. Suppose $b_{t}>a_{t}$. Let $S=\{i \in$ $\left.N: p(i)_{t} \leq \alpha\right\}$. Since $\varphi$ is uncompromising, $\varphi\left(a^{S}, b^{N \backslash S}\right)_{t}=a_{t}$. Hence $\varphi_{\gamma}\left(a^{S}, b^{N \backslash S}\right)_{t}=a_{t}$. On the other hand, since $\varphi_{\gamma}$ is uncompromising (see lemma 5.3), $\varphi_{\gamma}\left(a^{S}, b^{N \backslash S}\right)_{t}=b_{t}$. So we have a contradiction. Therefore, $b_{t} \leq a_{t}$. Similarly we can prove that $a_{t} \leq b_{t}$. Consequently, $\varphi(p)_{t}=\varphi_{\gamma}(p)_{t}$.

We can now characterize all uncompromising schemes: 
Theorem 5.2 Let $\varphi: A^{N} \rightarrow A$ be a voting scheme. Then $\varphi$ is uncompromising if, and only if, there exists an assignment of disagreements $\gamma$ such that $\varphi$ coincides with the standard coordinatewise veto scheme $\varphi_{\gamma}$.

Proof The if-part is lemma 5.3. The only-if-part follows by lemmas 5.7 and 5.8 .

Theorem 5.2 generalizes proposition 4 in Border and Jordan (1983) to the multidimensional case. Note further that we allow the set of alternatives $A$ to be a nonempty open subset, whereas Border and Jordan assume that $A=\mathbf{R}^{m}$ (with $m=1$ ).

Next, we turn to strategy-proofness. As was demonstrated in lemma 5.2, every scheme $\varphi_{\gamma}$ is strategy-proof with respect to the large class $P^{*}$. It will be shown that also the converse holds, by requiring $\varphi$ to be strategy-proof with respect to a specific subclass of $P^{*}$.

In order to define this subclass, let, for every $t \in M, a \in A$, and $\alpha>0$, the function $f^{t, a, \alpha}: A \rightarrow \mathbf{R}$ be defined by

$$
f^{t, a, \alpha}(x):=\left\{\begin{array}{l}
a_{t}-x_{t}+1 \text { if } x_{t} \leq a_{t} \text { and } x \neq a \\
\alpha\left(x_{t}-a_{t}\right)+1 \text { if } x_{t}>a_{t} \\
0 \text { if } x=a
\end{array}\right.
$$

In figure 5.1 we sketched some $f^{t, a, \alpha}(x)$ as function of $x_{t}$. As we can see, $f^{t, a, \alpha}(x)$ is minimal if $x_{t}=a_{t}$, whereas $\alpha$ determines the right slope of the function.

For $\alpha>0$ let $P_{\alpha}$ denote the class of preferences $R$ over $A$ such that: $R \in$ $P_{\alpha} \Leftrightarrow \exists a \in A, t \in M\left[\forall x, y \in A:\left\langle x, y>\in R \Leftrightarrow f^{t, a, \alpha}(x) \leq f^{t, a, \alpha}(y)\right]\right.$. Clearly, if $R \in P_{\alpha}$, then the point $a$ as in this definition is the unique best element of $R$, implying that (R1) is fulfilled by $R$. It is also obvious that (R2) holds for $R$, hence $P_{\alpha} \subset P^{*}$.

We now have:

Lemma 5.9 Let the scheme $\varphi: A^{N} \rightarrow A$ be strategy-proof with respect to $P_{\alpha}$, for every $\alpha>0$. Then $\varphi$ is uncompromising.

Proof Let $t \in M$ and $i \in N$, and $p, q \in A^{N}$ with $p(j)=q(j)$ for all $j \neq i$. Suppose $p(i)_{t}<\varphi(p)_{t}$ and $q(i)_{t} \leq \varphi(p)_{t}$. Observe that it suffices to prove that $\varphi(p)_{t} \leq \varphi(q)_{t}$. Namely, this implies $\varphi(p)_{t}=\varphi(q)_{t}$, because $\varphi(p)_{t}<\varphi(q)_{t}$ would imply $\varphi(q)_{t} \leq \varphi(p)_{t}$ and hence a contradiction, by interchanging the roles of $p$ and $q$. 


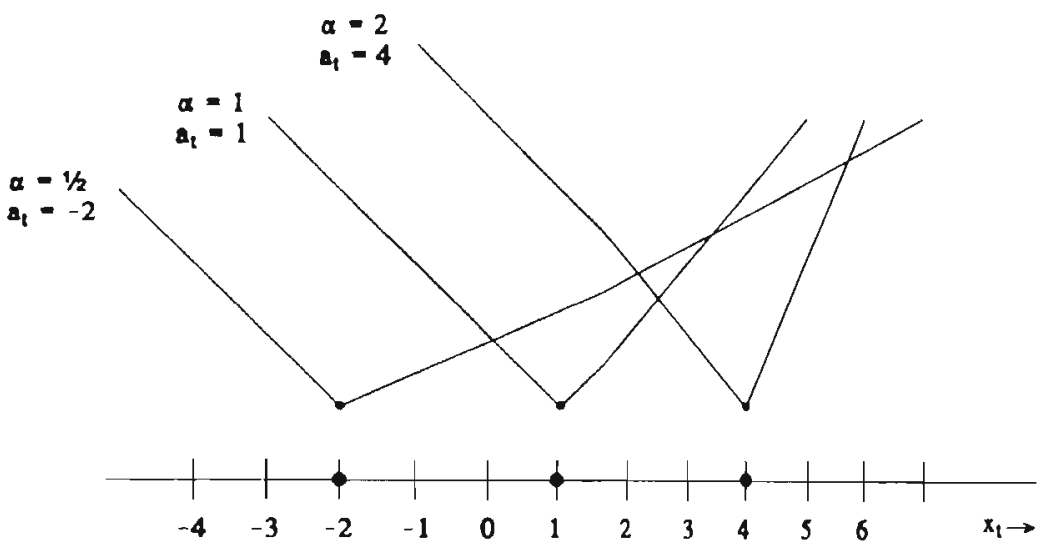

Figure 5.1: $f^{t, a, \alpha}(x)$ as function of $x_{t}$ for several $a, \alpha$.

Since $\varphi$ is strategy-proof with respect to $P_{\alpha}$ for all $\alpha>0$, it follows that $f^{t, p(i), \alpha}(\varphi(p)) \leq f^{t, p(i), \alpha}(\varphi(q))$ for all $\alpha>0$. There are two cases:

Case (i): $p(i)_{t} \leq \varphi(q)_{t}$.

Then $\alpha\left(\varphi(q)_{t}-p(i)_{t}\right) \geq \alpha\left(\varphi(p)_{t}-p(i)_{t}\right)$ for all $\alpha>0$, hence $\varphi(q)_{t} \geq \varphi(p)_{t}$. Case (ii): $p(i)_{t}>\varphi(q)_{t}$.

Then $p(i)_{t}-\varphi(q)_{t} \geq \alpha\left(\varphi(p)_{t}-p(i)_{t}\right)$ for all $\alpha>0$, which is clearly impossible.

Lemmas 5.2 and 5.9 , and theorem 5.2 imply:

Corollary 5.1 The scheme $\varphi: A^{N} \rightarrow A$ is strategy-proof with respect to $P^{*}$ if, and only if, there is an assignment of disagreements $\gamma$ such that $\varphi=\varphi_{\gamma}$.

An alternative proof of corollary 5.1 could be obtained by showing that a scheme which is strategy-proof with respect to $P^{*}$, is determined coordinatewise, for instance by considering the class $P_{\alpha}$, and then applying Proposition 3 in Moulin (1980), suitably modified to hold for arbitrary subsets of the real line. The above proof, however, is self-contained. Moreover, theorem 5.2 is of considerable interest of its own, and will be used in the next section. 


\subsection{Special cases}

In the preceding section it was proved that every scheme which is strategyproof with respect to the class of preferences $P^{*}$, is a coordinatewise veto scheme (corollary 5.1). For this result, only strategy-proofness with respect to the subclasses $P_{\alpha}$ was needed (see lemma 5.9). Depending on the conditions imposed on the set of alternatives $A$, other possibly weaker strategyproofness requirements may suffice. For instance, if $A$ is a bounded set then strategy-proofness with respect to $P_{\alpha}$ for only one value of $\alpha$ (chosen sufficiently large) already implies that the scheme must be uncompromising, and therefore must be a coordinatewise veto scheme. This can be seen by going over the proof of lemma 5.9 .

In subsection 5.5.1, a set of conditions on the set $A$ will be formulated such that strict strategy-proofness with respect to the class $P^{1}$ combined with strategy-proofness with respect to the subclass $P^{\infty}$ (see section 5.2), suffices to determine the scheme as a coordinatewise veto scheme. Also, an example of a set $A$ will be considered where strict strategy-proofness with respect to $P^{1}$ alone is sufficient to fix the scheme as a coordinatewise veto scheme.

In subsection 5.5.2, we first demonstrate that strategy-proofness with respect to, for instance, the class of all separable quadratic preferences - a class often considered in the literature, see for example Border and Jordan (1983) - implies strategy-proofness with respect to the class $P_{1}$ (see section 5.4). Additional conditions on the set of alternatives and the scheme will be formulated to guarantee that strategy-proofness with respect to $P_{1}$ determines the scheme as a coordinatewise veto scheme.

\subsubsection{The subclasses $P^{1}$ and $P^{\infty}$}

We start by formulating two conditions on the set of alternatives $A \subset \mathbf{R}^{m}$. Let $B \subset A$ and $x, y \in B$. Then $x$ and $y$ are called arcconnected in $B$ if there exists a continuous function $f:[0,1] \rightarrow B$ with $f(0)=x$ and $f(1)=y$. The set $B$ is arcconnected if $x$ and $y$ are arcconnected for all $x, y \in B$. The first condition is:

(A1) For all $t \in M$ and $\alpha \in \mathbf{R}$, the sets $\left\{a \in A: a_{t}>\alpha\right\}$ and $\left\{a \in A: a_{t}<\right.$ $\alpha\}$ are arcconnected.

Condition (A1) will be needed in order to apply strict strategy-proofness with respect to $P^{1}$ in the theorem below. The following condition will be 
needed in order to apply strategy-proofness with respect to $p^{\infty}$. For all $x, y \in A$, the $x$-orthant of $y$ is the set.

$$
\operatorname{ort}(x, y):=\left\{z \in A: \forall t \in M\left[z_{t} \geq x_{t} \Leftrightarrow y_{t} \geq x_{t}\right]\right\} .
$$

(A2) Let $x, y \in A, \emptyset \neq M^{\prime} \subset M$ such that $x_{s}=y_{s}$ for all $s \in M^{\prime}$, and $t \in M \backslash M^{\prime}$. Then $y=x$ or there is a $z$ arcconnected to $y$ in ort $(x, y)$ such that $\left|z_{t}-x_{t}\right|>\left|z_{s}-x_{s}\right|$ for all $s \in M, s \neq t$.

Conditions (A1) and (A2) are fairly technical, but there are many interesting sets $A$ satisfying both conditions. If $m=2$, then (A2) is trivially satisfied (take $z=y$ ) and therefore presents no restriction. Thus, if $m=2$, for instance all convex sets satisfy (A1) and (A2), but also many networks or graphs belong to this category. If $m>2$, then a rectangular subset of the form $\left\{z \in \mathbf{R}^{m}: \forall t \in M\left[\min \left\{x_{t}, y_{t}\right\} \leq z_{t} \leq \max \left\{x_{t}, y_{t}\right\}\right]\right\}$ for certain $x, y \in \mathbf{R}^{m}$, with possibly some disjoint open convex subsets from its interior left out, satisfies (A1) and (A2). Also the idealized network considered in theorem 5.4 fulfills both conditions. Consequently, the following theorem applies to many interesting cases.

Theorem 5.3 Let $A$ be a closed subset of $\mathbb{R}^{m}$ satisfying (A1) and (A2). Let $\varphi: A^{N} \rightarrow A$ be a scheme. Then the following two statements are equivalent:

(i) $\varphi$ is strictly strategy-proof with respect to $P^{1}$ and strategy-proof with respect to $p^{\infty}$.

(ii) There is an assignment of disagreements $\gamma$ such that $\varphi=\varphi_{\gamma}$.

Before proving this theorem, we need a few lemmas. The first lemma is concerned with continuity of $\varphi$. It is quite similar to lemma 2.16 .

Lemma 5.10 Let $\|\cdot\|$ be a norm on $\mathbb{R}^{m}$. Let $A \subset \mathbf{R}^{m}$ be closed. Let $\varphi: A^{N} \rightarrow A$ be strictly strategy-proof with respect to $\|\cdot\|$. Then $\varphi$ is componentwise continuous (with respect to $\|\cdot\|$ ).

Proof We will prove that $\varphi$ is continuous in the first component. Let $p^{0}, p^{1}, p^{2}, \ldots \in A^{N}$ with $p^{0}={ }_{N \backslash\{1\}} p^{k}$ for every $k \in \mathbb{N}$ and $\left\|p^{k}(1)-p^{0}(1)\right\| \downarrow 0$. Then by lemma 3.6 (i), $\varphi\left(p^{k}\right)$ has a limit point in $\mathbf{R}^{m}$, say $v$. Since $A$ is closed, $v \in A$. Lemma 3.6 (iii) implies that $\left\|p^{0}(1)-\varphi\left(p^{0}\right)\right\|=\left\|p^{0}(1)-v\right\|$. Hence by SSP, $v=\varphi\left(p^{0}\right)$. Therefore $\varphi$ is continuous in the first component. Similarly, we can prove that $\varphi$ is continuous in the other components. Consequently, $\varphi$ is componentwise continuous. 
The following lemma states, roughly, that in case of strict strategy-proofness with respect to $\|\cdot\|_{1}$, changes of the profile within the same scheme point orthant, do not affect the compromise point.

Lemma 5.11 Let $A$ be closed. Let $\varphi: A^{N} \rightarrow A$ be strictly strategy-proof with respect to $P^{1}$. Let $x, y \in A, i \in N$ and $p, q \in A^{N}$ such that $p(i)=x$, $q(i)=y, p=N /\{i\}, x \neq \varphi(p)$ and $x$ and $y$ are arcconnected in $\operatorname{ort}(\varphi(p), x)$. Then $\varphi(q)=\varphi(p)$.

Proof First observe that, if $x=\varphi(p)$, then $y=\varphi(p)=x$ and we are done. So suppose $x \neq \varphi(p)$. Let $f:[0,1] \rightarrow \operatorname{ort}(\varphi(p), x)$ be a continuous function with $f(0)=x$ and $f(1)=y$. For every $\alpha \in[0,1]$ let $p^{\alpha} \in A^{N}$ be defined by $p^{\alpha}(i):=f(\alpha)$ and $p^{\alpha}(j):=p(j)$ for all $j \neq i$. Let $\psi:[0,1] \rightarrow A$ with $\psi(\alpha):=\varphi\left(p^{\alpha}\right)$. By lemma 5.10 and the continuity of $f, \psi$ is continuous. Let $\mu:=\min \left\{\left|x_{t}-\varphi(p)_{t}\right|: x_{t} \neq \varphi(p)_{t}, t \in M\right\}$. Since $x \neq \varphi(p), \mu>0$. By the continuity of $\psi$ we can define $\beta \in[0,1]$ such that $\psi(\beta) \neq \varphi(p)$ and $\|\psi(\beta)-\varphi(p)\|_{1} \leq \mu$. Hence,

$$
\left|\psi(\beta)_{t}-\varphi(p)_{t}\right| \leq \mu \text { for all } t \in M .
$$

We will derive a contradiction, by which the proof is completed. Define

$$
\begin{aligned}
& M_{1}:=\left\{t \in M: x_{t}>\varphi(p)_{t}\right\} \\
& M_{2}:=\left\{t \in M: x_{t}<\varphi(p)_{t}\right\} \\
& M_{3}:=\left\{t \in M: x_{t}=\varphi(p)_{t}\right\} .
\end{aligned}
$$

By strict strategy-proofness with respect to $\mathcal{P}^{1}$ we have

$$
\sum_{t \in M}\left|x_{t}-\varphi(p)_{t}\right|<\sum_{t \in M}\left|x_{t}-\psi(\beta)_{t}\right| .
$$

The definition of $\mu$ and (5.4) imply

$$
x_{t} \geq \psi(\beta)_{t} \text { if } t \in M_{1}, x_{t} \leq \psi(\beta)_{t} \text { if } t \in M_{2} .
$$

By (5.5) and (5.6):

$$
\begin{aligned}
\sum_{t \in M} \mid x_{t}-\varphi(p)_{\mid t}< & \sum_{t \in M_{1}}\left|x_{t}-\varphi(p)_{t}\right|+\varphi(p)_{t}-\psi(\beta)_{t} \\
& +\sum_{t \in M_{2}}\left|x_{t}-\varphi(p)_{t}\right|-\varphi(p)_{t}+\psi(\beta)_{t} \\
& \sum_{t \in M_{3}}\left|x_{t}-\varphi(p)_{t}\right|+\left|\varphi(p)_{t}-\psi(\beta)_{t}\right|
\end{aligned}
$$


hence:

$$
0<\sum_{t \in M_{1}}\left(\varphi(p)_{t}-\psi(\beta)_{t}\right)+\sum_{t \in M_{2}}\left(\psi(\beta)_{t}-\varphi(p)_{t}\right)+\sum_{t \in M_{3}}\left|\varphi(p)_{t}-\psi(\beta)_{t}\right|
$$

Strict strategy-proofness and $f(\beta) \in \operatorname{ort}(\varphi(p), x)$ imply:

$$
\begin{aligned}
\sum_{t \in M}\left|f(\beta)_{t}-\varphi(p)_{t}\right|> & \sum_{t \in M}\left|f(\beta)_{t}-\psi(\beta)_{t}\right| \\
= & \sum_{t \in M_{1}}\left|f(\beta)_{t}-\varphi(p)_{t}\right|+\varphi(p)_{t}-\psi(\beta)_{t} \mid \\
& +\sum_{t \in M_{2}}|| f(\beta)_{t}-\varphi(p)_{t}\left|-\varphi(p)_{t}+\psi(\beta)_{t}\right| \\
& +\sum_{t \in M_{3}}\left|\varphi(p)_{t}-\psi(\beta)_{t}\right| .
\end{aligned}
$$

Combining (5.7) and (5.8) yields

$$
\begin{aligned}
& \sum_{t \in M_{1}} \varphi(p)_{t}-\psi(\beta)_{t}+\left|f(\beta)_{t}-\varphi(p)_{t}\right| \\
+ & \sum_{t \in M_{2}} \psi(\beta)_{t}-\varphi(p)_{t}+\left|f(\beta)_{t}-\varphi(p)_{t}\right|> \\
& \sum_{t \in M_{1}}\left|\varphi(p)_{t}-\psi(\beta)_{t}+\right| f(\beta)_{t}-\varphi(p)_{t}|| \\
+ & \sum_{t \in M_{2}}\left|\psi(\beta)_{t}-\varphi(p)_{t}+\right| f(\beta)_{t}-\varphi(p)_{t}||,
\end{aligned}
$$

which is the desired contradiction.

The next lemma shows that statement (i) in theorem 5.3 implies uncompromisingness of the scheme $\varphi$.

Lemma 5.12 Let $A$ be a subset of $\mathbb{R}^{m}$. Let $\varphi: A^{N} \rightarrow A$ be strictly strategyproof with respect to $P^{1}$. Suppose that for all $t \in M$ and all $p, q \in A^{N}$ satisfying for all $i \in N$ :

$$
\begin{aligned}
& p(i)_{t}<\varphi(p)_{t} \Rightarrow q(i)_{t}<\varphi(p)_{t} \\
& p(i)_{t}>\varphi(p)_{t} \Rightarrow q(i)_{t}>\varphi(p)_{t}
\end{aligned}
$$

we have: $\varphi(p)_{t}=\varphi(q)_{t}$. Then $\varphi$ is uncompromising. 
Proof Let $i \in N, t \in M$, and $p, q \in A^{N}$ with $p={ }_{N \backslash\{i\}} q, p(i)={ }_{M \backslash\{t\}} q(i)$, $q(i)_{t}=\varphi(p)_{t}$. It is sufficient to prove that $\varphi(q)=\varphi(p)$. By strategyproofness, $\|\varphi(p)-p(i)\|_{1} \leq\|\varphi(q)-p(i)\|_{1}$ and $\|\varphi(q)-q(i)\|_{1} \leq \| \varphi(p)-$ $q(i) \|_{1}$. Adding these inequalities, we obtain $\left|\varphi(q)_{t}-q(i)_{t}\right|+\left|\varphi(p)_{t}-p(i)_{t}\right| \leq$ $\left|\varphi(q)_{t}-p(i)_{t}\right|$. By the triangle inequality we have

$$
\begin{aligned}
\left|\varphi(q)_{t}-p(i)_{t}\right| & =\left|\varphi(q)_{t}-q(i)_{t}+\varphi(p)_{t}-p(i)_{t}\right| \\
& \leq\left|\varphi(q)_{t}-q(i)_{t}\right|+\left|\varphi(p)_{t}-p(i)_{t}\right|
\end{aligned}
$$

so all inequalities must be equalities. In particular this implies $\| \varphi(p)-$ $p(i)\left\|_{1}=\right\| \varphi(q)-p(i) \|_{1}$, so $\varphi(p)=\varphi(q)$ by strict strategy-proofness.

Lemma 5.13 Let $A$ be a closed subset of $\mathbf{R}^{m}$ satisfying (A1) and (A2). Let $\varphi: A^{N} \rightarrow A$ be strictly strategy-proof with respect to $P^{1}$ and strategy-proof with respect to $P^{\infty}$. Then $\varphi$ is uncompromising.

Proof Let $i \in N, t \in M$, and $p, q \in A^{N}$ with $p={ }_{N /\{i\}} q, p(i)_{t}>\varphi(p)_{t}$, $q(i)_{t}>\varphi(p)_{t}$. In view of lemma 5.12 , it is by symmetry sufficient to prove that $\varphi(p)_{t} \geq \varphi(q)_{t}$.

By (A1), there is a continuous function $f:[0,1] \rightarrow\left\{x \in A: x_{t}>\varphi(p)_{t}\right\}$ with $f(0)=p(i)$ and $f(1)=q(i)$. For every $\alpha \in[0,1]$ define the profile $p^{\alpha} \in A^{N}$ by $p^{\alpha}(j):=p(j)$ for all $j \neq i$ and $p^{\alpha}(i):=f(\alpha)$. Define $\psi:[0,1] \rightarrow$ $A$ by $\psi(\alpha):=\varphi\left(p^{\alpha}\right)$ for every $\alpha \in[0,1]$. By lemma $5.10, \psi$ is continuous. Therefore, the set $V:=\left\{\alpha \in[0,1]: \psi(\alpha)_{t} \leq \varphi(p)_{t}\right\}$ is closed. It is sufficient to prove that $1 \in V$. In order to show that $1 \in V$ it is sufficient to prove that for every $\alpha \in V, \alpha<1$, there is an $\varepsilon>0$ with $\alpha+\varepsilon \in V$. So let $\alpha \in V$, $\alpha<1$.

If there is a $\delta>0$ with $f([\alpha, \alpha+\delta)) \subset \operatorname{ort}(\psi(\alpha), f(\alpha))$, then $\psi(\alpha+\varepsilon)=$ $\psi(a)$ for every $0<\varepsilon<\delta$ by lemma 5.12. So in that case, we are done.

If such a $\delta$ does not exist, then there is a nonempty subset $M^{\prime}$ of $M$ with $f(\alpha)_{s}=\psi(\alpha)_{s}$ for all $s \in M^{\prime}$, and a $\delta^{\prime}>0$ with $f(\alpha+\varepsilon)_{s} \neq f(\alpha)$, for all $s \in M^{\prime}$ and $0<\varepsilon<\delta^{\prime}$. With $M^{\prime}$ and $\delta^{\prime}$ like this, take $z$ arcconnected to $f(\alpha)$ in $\operatorname{ort}(\psi(\alpha), f(\alpha))$ with $\left|z_{t}-\psi(\alpha)_{t}\right|>\left|z_{s}-\psi(\alpha)_{s}\right|$ for all $s \in M \backslash\{t\}$. Such a point $z$ exists in view of (A2); note that $t \notin M^{\prime}$ because $f(\alpha)_{t}>$ $\varphi(p)_{t} \geq \psi(\alpha)_{t}$

Let $\tilde{p} \in A^{N}$ be defined by $\tilde{p}(j):=p(j)$ if $j \neq i, \tilde{p}(i):=z$. Then lemma 5.12 implies $\varphi(\tilde{p})=\varphi\left(p^{\alpha}\right)=\psi(\alpha)$. Strategy-proofness with respect to ${ }^{\infty}$ implies $\|z-\psi(\alpha)\|_{\infty} \leq\|z-\psi(\alpha+\varepsilon)\|_{\infty}$ for all $0<\varepsilon<\delta^{\prime}$. By the choice of $z$, we have $\|z-\psi(\alpha)\|_{\infty}=\left|z_{t}-\psi(\alpha)_{t}\right|$, and there exists $\delta^{\prime \prime}>0$ such that if $\|x-\psi(\alpha)\|_{\infty}<\delta^{\prime \prime}$, then $\|z-x\|_{\infty}=\left|z_{t}-x_{t}\right|$. 
By the continuity of $\psi$ there exists $0<\delta<\delta^{\prime}$ such that $0<\varepsilon<\delta$ implies $\| \psi(\alpha+\varepsilon)-\left.\psi(\alpha)\right|_{\infty}<\delta^{\prime \prime}$. Hence, $\left|z_{t}-\psi(\alpha)_{t}\right| \leq\left|z_{t}-\psi(\alpha+\varepsilon)_{t}\right|$ for all $0<\varepsilon<\delta$. Since $z \in \operatorname{ort}(\psi(\alpha), f(\alpha))$ and $f(\alpha)_{t}>\psi(\alpha)_{t}$, we have $z_{t}>\psi(\alpha)_{t}$. Hence, by continuity of $\psi, \psi(\alpha)_{t} \geq \psi(\alpha+\varepsilon)_{t}$ for $0<\varepsilon<\delta, \varepsilon$ small enough. We conclude that $\alpha+\varepsilon \in V$.

We are now sufficiently equipped to prove theorem 5.3.

Proof of theorem 5.3 The implication (ii) $\Rightarrow$ (i) follows from lemma 5.2. (Strict strategy-proofness is straightforward and left to the reader.) For the implication (i) $\Rightarrow$ (ii), note that $\varphi$ is uncompromising by lemma 5.13. Now (ii) follows from theorem 5.2 .

Given more specific information on the set $A$, the conditions in theorem 5.3 may be further relaxed. By way of an example, let $A \subset \mathbf{R}^{m}$ be a grid, i.e., for every $t \in M$ there is a natural number $m^{t}$ and a set $L^{t}=\left\{l_{1}^{t}, l_{2}^{t}, \ldots, l_{m}^{t}\right\} \subset \mathbf{R}$ with $l_{1}^{t}<\ldots<l_{m}^{t}$ such that

$$
A=\left\{x \in \mathbb{R}^{m}: \exists t \in M\left[l_{1}^{t} \leq x_{t} \leq l_{m^{2}}^{t} \& x_{s} \in L^{s} \text { for all } s \neq t\right]\right\}
$$

For such a grid, the following theorem holds.

Theorem 5.4 Let $A$ be a grid and $\varphi: A^{N} \rightarrow A$ a scheme. Equivalent are:

(i) $\varphi$ is strictly strategy-proof with respect to $p^{1}$.

(ii) $\varphi=\varphi_{\gamma}$ for some assignment of disagreements.

Proof Only the implication (i) $\Rightarrow$ (ii) needs attention. By theorem 5.2, it is sufficient to prove that $\varphi$ is uncompromising. Consider the proof of lemma 5.13, and observe that the first three paragraphs remain valid. (Observe, in particular, that a grid satisfies condition (A1).) We proceed as follows. We have

$$
\psi(\alpha)_{t} \leq \varphi(p)_{t}<f(\alpha)_{t}
$$

Suppose $f(\alpha)_{s} \neq \psi(\alpha)_{s}$ for some $s \in M$. Let $x \in A$ with $x_{r}:=f(\alpha)_{r}$ for all $r \neq s, x_{s}:=\psi(\alpha)_{s}$. Lemma 5.11 and the continuity of $\varphi$ (lemma 5.10) imply $\varphi(x)=\psi(\alpha)$. By repeated application of this argument, we obtain $\varphi(z)=\psi(\alpha)$ where $z \in A$ is determined by $z_{t}:=f(\alpha)_{t}$ and $z_{r}:=\psi(\alpha)_{r}$ for all $r \neq t$. By continuity of $\psi$, if $\varepsilon>0$ is small enough then $\psi(\alpha+\varepsilon)_{t}>\psi(\alpha)_{t}$ would imply that $\psi(\alpha+\varepsilon)$ is a point on the line segment with endpoints $\psi(\alpha)$ and $z$, since $A$ is a grid. In that case, $\|\psi(\alpha+\varepsilon)-z\|_{1}<\|\psi(\alpha)-z\|_{1}$ 
contradicting strategy-proofness of $\varphi$. We conclude that $\psi(\alpha+\varepsilon)_{t} \leq \psi(\alpha)_{t}$ for $\varepsilon>0$ small enough, hence $\alpha+\varepsilon \in V$ by (5.9).

Remark. (i) It should be noted that, although theorem 5.4 is not an impossibility theorem, it is quite close to one. Namely, in general, even if all the disagreement points are on the 'crosspoints' of the grid $A$, then for an arbitrary profile $p$ it will not be true that $\varphi_{\gamma}(p) \in A$, i.e., that $\varphi_{\gamma}$ is a scheme and not just a prescheme. An example of a nonconstant nondictatorial possibility is a scheme $\varphi_{\gamma}$ with all disagreement points on the same straight line in $A$.

(ii) A result similar to theorem 5.4 , for a set of alternatives consisting of the 'crosspoints' of a grid, is obtained in Barberà et al. (1990).

We conclude this subsection by two examples, showing that none of the two strategy-proofness conditions in theorem 5.3 can be left out.

Example 5.3 Let $m=2$ and $A=\mathbf{R}^{m}$. Let $N=\{1,2, \ldots, 2 l+1\}$ for some $l \in\{0,1,2, \ldots\}$. For every $x \in A$ let $f(x):=\frac{1}{2}\left(x_{1}+x_{2}\right)$, and let $\varphi: A^{N} \rightarrow A$ be defined by

$$
\varphi(p)_{1}:=\varphi(p)_{2}:=\operatorname{med}(f(p(1)), \ldots, f(p(2 l+1))) .
$$

Then $\varphi$ is strategy-proof with respect to $\rho^{k}$ for all $k \geq 1$ and $\rho \infty$, but not strictly strategy-proof with respect to $P^{1}$. Consequently, $\varphi$ is not a coordinatewise veto scheme.

Example 5.4 Let $m=2$ and $A=\left\{x \in \mathbb{R}^{2}: 0 \leq x_{1} \leq \pi\right\}$. Let $N=$ $\{1,2, \ldots, 2 l+1\}$ for some $l \in\{0,1,2, \ldots\}$. Let $\varphi: A^{N} \rightarrow A$ be defined by

$$
\begin{aligned}
& \varphi(p)_{1}:=\operatorname{med}\left(p(1)_{1}, p(2)_{1}, \ldots, p(2 l+1)_{1}\right), \\
& \varphi(p)_{2}:=\operatorname{med}\left(p(1)_{2}, p(2)_{2}, \ldots, p(2 l+1)_{2}\right)+\sin \varphi(p)_{1}
\end{aligned}
$$

for all $p \in A^{N}$. Then $\varphi$ is strictly strategy-proof with respect to $p^{1}$ but not strategy-proof with respect to $P^{\infty}$. Obviously, $\varphi$ is not a coordinatewise veto scheme.

\subsubsection{Separable and quadratic preferences}

Let $k \in \mathbf{N}$. A class of preferences often considered in the literature is the class

$$
\begin{aligned}
Q^{k} & :=\left\{R \subset A \times A: \exists x \in A, \alpha_{1}, \ldots, \alpha_{m}>0 \forall y, z \in A[(y, z) \in R\right. \\
& \left.\left.\Leftrightarrow \sum_{t \in M} \alpha_{t}\left|x_{t}-y_{t}\right|^{k} \leq \sum_{t \in M} \alpha_{t}\left|x_{t}-z_{t}\right|^{k}\right]\right\} .
\end{aligned}
$$


Note that $Q^{k} \subset P^{*}$, and that $P^{k} \subset Q^{k}$ by setting the weights $\alpha_{t}$ equal to 1. A special case is the class $Q^{2}$ of separable quadratic preferences, see in particular Border and Jordan (1983).

Intuitively, strategy-proofness with respect to some class $Q^{k}$ seems to be a strong condition, since it requires an individual not to profit from a unilateral deviation no matter how the coordinates of the alternatives in $A$ are weighted. This intuition is confirmed by the following lemma, in which the class of preferences $P_{\alpha}$ for $\alpha=1$ (see section 5.4, before lemma 5.9) plays a role.

Lemma 5.14 Let $\varphi: A^{N} \rightarrow A$ be a scheme, $k \in \mathbb{N}$, and suppose $\varphi$ is strategy-proof with respect to $Q^{k}$. Then $\varphi$ is strategy-proof with respect to $P_{1}$.

Proof Let $i \in N$ and $p, q \in A^{N}$ with $p(j)=q(j)$ for all $j \neq i$. Let $t \in M$. It is sufficient to prove that $\left|\varphi(p)_{t}-p(i)_{t}\right| \leq\left|\varphi(q)_{t}-p(i)_{t}\right|$. Let, for $l \in\{2,3, \ldots\}, \alpha^{l} \in \mathbf{R}^{m}$ be defined by $\alpha_{s}^{l}:=l^{-1}$ if $s \neq t, \alpha_{t}^{l}:=1-l^{-1}$. Strategy-proofness with respect to $Q^{k}$ implies for all l:

$$
\sum_{s \in M} \alpha_{s}^{l}\left|\varphi(p)_{s}-p(i)_{s}\right|^{k} \leq \sum_{s \in M} \alpha_{s}^{l}\left|\varphi(q)_{s}-p(i)_{s}\right|^{k}
$$

If $l \rightarrow \infty$, in the limit:

$$
\left|\varphi(p)_{t}-p(i)_{t}\right|^{k} \leq\left|\varphi(q)_{t}-p(i)_{t}\right|^{k}
$$

which implies the desired result.

Strategy-proofness of a scheme with respect to $P_{1}$ is a strong condition since, to a great extent, it determines the scheme coordinatewise. In order to make this more precise, call the set of alternatives $A$ coordinatewise midpoint closed if the following condition holds:

$$
\forall t \in M, \alpha, \beta \in \pi_{t}(A)\left[\frac{1}{2} \alpha+\frac{1}{2} \beta \in \pi_{t}(A)\right] .
$$

Coordinatewise midpoint closedness is a denseness condition on $A$.

Theorem 5.5 Let $\varphi: A^{N} \rightarrow A$ be a unanimous scheme, and let the set $A$ be coordinatewise midpoint closed. Then the following two statements are equivalent:

(i) $\varphi$ is strategy-proof with respect to $P_{1}$. 
(ii) $\varphi$ is a coordinatewise veto scheme.

An immediate consequence of lemma 5.14, theorem 5.5, and lemma 5.2 is:

Corollary 5.2 Let $\varphi$ be a unanimous scheme, let $A$ be coordinatewise midpoint closed, and let $k \in \mathbf{N}$. Then the following two statements are equivalent:

(i) $\varphi$ is strategy-proof with respect to $Q^{k}$.

(ii) $\varphi$ is a coordinatewise veto scheme.

This corollary extends theorem 1 in Border and Jordan (1983) to all classes of alternatives $A$ that are coordinatewise midpoint closed. Furthermore, our proof of this result seems to be more elementary and straightforward than Border and Jordan's proof.

Observe that the coordinatewise midpoint closedness condition admits many quite different sets of alternatives. For instance, convex or arcconnected sets are coordinatewise midpoint closed, but also the set $\mathbf{Q}^{m}$ (where $Q$ denotes the rational numbers).

In order to prove theorem 5.5 we need a few lemmas.

Lemma 5.15 Let $\varphi: A^{N} \rightarrow A$ be strategy-proof with respect to $P_{1}$. Let $p, q \in A^{N}, i \in N$ and $t \in M$ with $p(j)=q(j)$ for all $j \neq i$ and $p(i)_{t}<$ $q(i)_{t} \leq \varphi(p)_{t}$. Then $\varphi(q)_{t}=\varphi(p)_{t}$.

Proof Strategy-proofness with respect to $P_{1}$ implies $\left|\varphi(q)_{t}-q(i)_{t}\right| \leq$ $\left|\varphi(p)_{t}-q(i)_{t}\right|$ as well as $\left|\varphi(p)_{t}-p(i)_{t}\right| \leq\left|\varphi(q)_{t}-p(i)_{t}\right|$. This implies $\varphi(q)_{t}=\varphi(p)_{t}$.

Lemma 5.16 Let $A$ be coordinatewise midpoint closed. Let $\varphi: A^{N} \rightarrow A$ be unanimous and strategy-proof with respect to $P_{1}$. Then

$$
\min _{i \in N} p(i)_{t} \leq \varphi(p)_{t} \leq \max _{i \in N} p(i)_{t} \text { for all } p \in A^{N}, t \in M .
$$

Proof Let $p \in A^{N}$ and $t \in M$ with, say, $p(i)_{t}<\varphi(p)_{t}$ for all $i \in N$. It is sufficient to derive a contradiction. Let $j \in N$ such that $p(j)_{t} \geq p(i)_{t}$ for all $i \in N$. Take a point $x$ in $A$ with $p(j)_{t}<x_{t}<\varphi(p)_{t}$, such a point exists since $A$ is coordinatewise midpoint closed. Repeated application of lemma 5.15 yields $\varphi(q)_{t}=\varphi(p)_{t}$ for the profile $q$ with $q(N)=\{x\}$. Unanimity, however, implies $\varphi(q)_{t}=x_{t}<\varphi(p)_{t}$. This gives the desired contradiction. 
Lemma 5.17 Let $\varphi: A^{N} \rightarrow A$ be strategy-proof with respect to $P_{1}$, and such that

$$
\min _{i \in N} p(i)_{t} \leq \varphi(p)_{t} \leq \max _{i \in N} p(i)_{t} \text { for all } p \in A^{N}, t \in M .
$$

Let $A$ be coordinatewise midpoint closed. Let $p, q \in A^{N}, i \in N$ and $t \in M$ with $p(j)=q(j)$ for all $j \neq i$ and $\varphi(p)_{t}<p(i)_{t} \leq q(i)_{t}$. Then:

(i) $\varphi(q)_{t}=\varphi(p)_{t}$.

(ii) $\varphi$ is uncompromising.

Proof (i) Since $A$ is coordinatewise midpoint closed, it is without loss of generality to assume that $q(i)_{t}-p(i)_{t}<p(i)_{t}-\varphi(p)_{t}$. Let $S:=\{j \in N$ : $\left.p(j)_{t}>p(i)_{t}\right\}$, and $s:=|S|$. The proof proceeds by induction on $s$.

Suppose $s=0$. By strategy-proofness with respect to $P_{1}$, either $\varphi(q)_{t}=$ $\varphi(p)_{t}$ or $\varphi(q)_{t} \geq p(i)_{t}+\left(p(i)_{t}-\varphi(p)_{t}\right)$. Suppose the latter were the case. Then $q(i)_{t}<\varphi(q)_{t}$, hence $q(j)_{t}<\varphi(q)_{t}$ for all $j \in N$ because $s=0$. This contradicts the assumption that $\varphi(p)_{t} \leq \max _{i \in N} p(i)_{t}$ for all $p \in A^{N}, t \in M$. Therefore, $\varphi(q)_{t}=\varphi(p)_{t}$.

Our induction hypothesis is as follows. Let $r, r^{\prime} \in A^{N}, i \in N, t \in M$, $r(j)=r^{\prime}(j)$ for all $j \neq i$, and $\varphi(r)_{t}<r(i)_{t} \leq r^{\prime}(i)_{t}$. Let further $\mid\{j \in N$ : $\left.r(j)_{t}>r(j)_{t}\right\} \mid<s$ where $s \geq 1$. Then $\varphi\left(r^{\prime}\right)_{t}=\varphi(r)_{t}$.

Next, let $|S|=8$. As before, by strategy-proofness with respect to $P_{1}$,

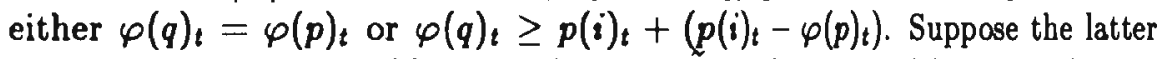
were the case. Then $q(i)_{t}<\varphi(q)_{t}$. Let $\delta:=\left\{j \in N: p(j)_{t} \geq \varphi(q)_{t}\right\} \subset S$. Consider $\tilde{p} \in A^{N}$ with $\tilde{p}(j):=p(j)$ if $j \notin \tilde{S}, \tilde{p}(j)_{t}:=q(i)_{t}$ if $j \in \tilde{S}$. By repeated application of lemma $5.15, \varphi(\tilde{p})_{t}=\varphi(p)_{t}$. Consider $\tilde{q} \in A^{N}$ with $\tilde{q}(j):=\tilde{p}(j)$ for $j \neq i$ and $\tilde{q}(i)_{t}:=q(i)_{t}$. By a similar proof as for the case $s=0$, it follows that $\varphi(\tilde{q})_{t}=\varphi(\tilde{p})_{t}$. Let $\tilde{S}=\left\{j_{1}, j_{2}, \ldots, j_{l}\right\}$. Note that $l \leq s$. Define profiles $q^{0}:=\tilde{q}$ and, for every $k=1,2, \ldots, l, q^{k}$ by $q^{k}(j):=q^{k-1}(j)$ if $j \neq j_{k}, q^{k}\left(j_{k}\right):=p\left(j_{k}\right)=q\left(j_{k}\right)$. Then $q^{l}=q$. By repeated application of the induction hypothesis, $\varphi\left(q^{0}\right)_{t}=\varphi\left(q^{1}\right)_{t}=\ldots=\varphi\left(q^{l}\right)_{t}$. Hence, $\varphi(\tilde{q})_{t}=\varphi(q)_{t}$. So $\varphi(q)_{t}=\varphi(p)_{t}$, as desired.

(ii) Follows by (i) and lemma 5.15 .

We are now in a position to prove theorem 5.5 .

Proof of theorem 5.5 The implication (ii) $\Rightarrow$ (i) follows by lemma 5.2. Assume (i). Then $\varphi$ is uncompromising by lemmas 5.16 and 5.17. Then (ii) follows from theorem 5.2. 


\subsection{Conclusion}

In this chapter we study voting schemes $\varphi: A^{N} \rightarrow A$, where $A$ is a nonempty subset of $\mathbf{R}^{m}$ and $\varphi$ satisfies strategy-proofness with respect to subclasses of the preference class $P^{*}$ (defined at the beginning of section 5.2). As $P^{*}$ contains many preferences, strategy-proofness with respect to $P^{*}$ is a very restrictive condition.

Because of this we are able to give a complete characterization of all voting schemes which satisfy SP with respect to $P^{*}$, provided that $A$ is open.

In section 5.5 we study some subclasses of $P^{*}$ and assume $A$ to have a special structure. Again, all voting schemes which are strategy-proof with respect to these subclasses appear to be standard coordinatewise veto schemes. Note that the classes of voting schemes we characterized in the chapters 2 , 3,4 are also subclasses of the class of coordinatewise veto schemes (except for the one person case studied in section 4.1). So one might wonder: how far can we relax certain conditions (delete anonymity for instance), such that all voting schemes satisfying these conditions are coordinatewise veto schemes? 



\section{Chapter 6}

\section{Independence of Irrelevant Alternatives}

This chapter is based on Peters, Van der Stel and Storcken (1992b).

\subsection{Introduction}

In this chapter we consider so-called preference schemes, also known as social welfare functions. Whereas a voting scheme assigns a compromise point to each possible profile of individually reported points, a preference scheme assigns a collective preference over the alternative set to each possible profile of individually reported preferences.

A preference scheme may satisfy a condition called independence of irrelevant alternatives (IIA), first introduced by Arrow (1963). This means that the collective preference between alternatives $x$ and $y$ is determined by the individually reported preferences between these two alternatives.

It is a well-known fact from the theory of social choice that, under Pareto optimality and in the classical discrete framework with all linear or weak orders allowed, the IIA-condition is logically equivalent to the strategyproofness condition of Gibbard (1973) and Satterthwaite (1975). See, for instance, the last mentioned reference. Thus, it is not surprising that impossibilities emerged in both cases.

In particular during the last decade, starting with the work of Laffond (1980) and Moulin (1980), models were studied in which the space of alternatives was extended to a continuum, whereas the domain of preferences was restricted. This made the theory of social choice easier to apply to stan- 
dard micro-economic models, and also led to a number of possibility results, i.e., the existence of nonconstant nondictatorial mechanisms. The preceding chapters of this dissertation are contributions in this area.

In this chapter it will be shown that, in models with a continuum of alternatives and restricted domains of preferences, IIA is, in general, much stronger than strategy-proofness. The intuition behind this result is that, since IIA is a requirement about preferences and not only about the best elements according to those preferences, the presence of a continuum of alternatives makes IIA very strong.

The organization of this chapter is as follows. Section 6.2 gives the model, and the main concepts and definitions, as far as they are not given by section 1.2. In section 6.3 strategy-proofness is derived from ILA. Section 6.4 shows that, already for a very restricted domain of preferences, IIA implies constantness or dictatoriality. Section 6.5 concludes.

\subsection{Preliminaries}

Let $m \geq 1$, and let $A$ be an open subset of $R^{m}$. We define $\hat{\rho}$ to be the class of all preferences $R$ on $A$ such that there is an $x \in A$ with for all $y \in A$

(A1) $(v, y) \in R$ for all $v \in \operatorname{box}(x, y)$,

(A2) $(y, w) \notin R$ for all $w \in \operatorname{box}(x, y) \backslash\{y\}$.

Lemma 6.1 Let $R \in \hat{\mathcal{P}}$. Then $|\operatorname{best}(R)|=1$.

Proof Let $R \in \hat{P}$. Then by (A1) we can take $x \in A$ such that $(v, y) \in R$ for all $v, y \in A$ with $v \in \operatorname{box}(x, y)$. Since $x \in \operatorname{box}(x, y)$ for all $y \in A$, $(x, y) \in R$ for all $y \in A$. Hence, $x \in \operatorname{best}(R)$. Since $x \in \operatorname{box}(x, y) \backslash\{y\}$ for all $y \in A \backslash\{x\},(\mathrm{A} 2)$ implies that $(y, x) \notin R$ for all $y \in A \backslash\{x\}$. So $y \notin \operatorname{best}(R)$ for all $y \in A \backslash\{x\}$. Consequently, $|\operatorname{best}(R)|=|\{x\}|=1$.

Thus every preference $R \in \hat{\mathcal{P}}$ has a unique best element, and preference is strictly decreasing when moving away, in any coordinate, from this best element. Henceforth, best $(R)$ will denote the unique best element of a preference $R$ in $\hat{P}$. From the definition it is clear that $\hat{P} \subset P^{*}$. The inclusion is strict: $P^{*}$ contains also preferences with more than one best element, and preferences which are not strictly decreasing when moving away coordinatewise from a best element. Note that $\hat{p}$ is nonstandard in the terminology of section 1.2, it contains also preferences which are not transitive. 
If $P$ is induced by a norm $\|\cdot\|$ on $\mathbb{R}^{m}$ which is such that the standard basis is $\|\cdot\|$-orthogonal, then $P$ is a subclass of $\hat{P}$. So $P^{p}$ (the class of preferences induced by the norm $\|\cdot\|_{p}$, see section 5.2) is a subclass of $\hat{\mathcal{P}}$ for every $p \in[1, \infty]$. So in particular $p^{2}$, the class of Euclidean preferences as discussed in chapters 3 and 4 , is a subclass of $\hat{P}$. Other examples of subclasses of $\hat{P}$ are the classes $Q^{k}$ (defined in subsection 5.5.2) with $k \in(0, \infty)$.

A class of preferences $P$ is called full if $\cup_{R \in P}$ best $(R)=A$. Note that $P^{k}$ and $Q^{k}$ are full for all $k \in[1, \infty)$. Let $P, P^{\prime}$ be subclasses of $\hat{P}$. Elements of $P^{N}$, i.e. maps assigning to each individual a preference in $P$, are called preference profiles. A function $F$ from $P^{N}$ to $P^{\prime}$ is called a preference scheme. A preference scheme $F$ assigns to every preference profile of individual preferences $r \in P^{N}$ a collective preference $F(r)$.

For preferences $R, R^{\prime}$ we denote $R={ }_{(x, y)} R^{\prime}$ if $R \cap((x, y) \times(x, y))=$

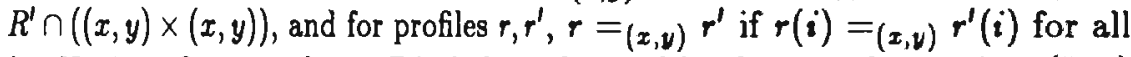
$i \in N$. A preference scheme $F$ is independent of irrelevant alternatives (IIA) if, for all $x, y \in A$ and all $r, r^{\prime} \in P^{N}$ : If $r={ }_{(x, y)} r^{\prime}$ then $F(r)={ }_{(x, y)} F\left(r^{\prime}\right)$. So if $F$ satisfies IIA, then the collective preference between alternatives $x, y$ is determined by the individual preferences between these two alternatives.

In the next section we associate a voting scheme with each IIA preference scheme, and derive strategy-proofness of such a scheme.

\subsection{IIA and strategy-proofness}

The following lemma states that, for IIA preference schemes, the best element of a collective preference depends only on the best elements of the individual preferences.

Lemma 6.2 Let $P, p^{\prime}$ be subclasses of $\hat{P}$. Let $i \in N$ and $r, r^{\prime} \in P^{N}$ with $r={ }_{N \backslash\{i\}} r^{\prime}$ and best $(r(i))=\operatorname{best}\left(r^{\prime}(i)\right)$. Let $F: P^{N} \rightarrow P^{\prime}$ satisfy IIA. Then $\operatorname{best}(F(r))=\operatorname{best}\left(F\left(r^{\prime}\right)\right)$.

Proof Without loss of generality suppose best $(F(r))=0 \in \mathbf{R}^{m}$, and $\operatorname{best}(r(i))=\operatorname{best}\left(r^{\prime}(i)\right)=x \geq 0$. Let $v:=\operatorname{best}\left(F\left(r^{\prime}\right)\right)$, suppose $v \neq 0$, and let $t \in M$ with $v_{t} \neq 0$. Let $B$ be a neighborhood of 0 in $A$. Choose $y, z \in B$ as follows. For all $s \neq t$ let $y_{s}=z_{s}=0$. Further:

(a) If $v_{t}<0$ take $v_{t}<y_{t}<z_{t}<0$.

(b) If $v_{t}>0$ and $x_{t}>0$ take $0<z_{t}<y_{t}<\min \left\{x_{t}, v_{t}\right\}$.

(c) If $v_{t}>0$ and $x_{t}=0$ take $0<z_{t}<y_{t}<v_{t}$.

Then, in all three cases, by (A1) and (A2), $r=(z, y)$ r', whereas $F(r) \neq(z, y)$ 
$F\left(r^{\prime}\right)$. This contradicts IIA of $\mathrm{F}$.

Lemma 6.2 expresses tops-onlyness: the best point of the collective preference is determined by the best points of the individually reported preferences. This makes it possible to associate a voting scheme to a preference scheme in a unique way. Let $F$ be an IIA-scheme as in the foregoing lemma, with the additional requirement that $\mathcal{P}$ is full. Then $\varphi_{F}: A^{N} \rightarrow A$ given by $\varphi_{F}(p):=\operatorname{best}(F(r))$ for some profile $r \in \mathcal{P}^{N}$ with $p(i)=$ best $(r(i))$ for all $i \in N$, is well-defined. By definition, $\varphi_{F}$ is a voting scheme.

Uncompromisingness was defined in section 5.2. We will show that each scheme $\varphi_{F}$ derived from an IIA preference scheme $F$ as above, is uncompromising. Using theorem 5.2, this implies that such a scheme must be a standard coordinatewise veto scheme. So by lemma 5.2 we have that IIA implies strategy-proofness.

Theorem 6.1 Let $P, P^{\prime}$ be subclasses of $\hat{P}$ with $P$ full, and let $F: P^{N} \rightarrow P^{\prime}$ satisfy IIA. Then $\varphi_{F}$ is uncompromising.

Proof Let $p, q \in A^{N}, i \in N$ and $t \in M$ be such that $p={ }_{N \backslash\{i\}} q, p(i)_{t}>$ $\varphi_{F}(p)_{t}$ and $q(i)_{t} \geq \varphi_{F}(p)_{t}$. It is sufficient to prove that $\varphi_{F}(p)_{t}=\varphi_{F}(q)_{t}$. By symmetry, it is sufficient to prove that $\varphi_{F}(p)_{t} \geq \varphi_{F}(q)_{t}$ (if $\varphi_{F}(p)_{t}>$ $\varphi_{F}(q)_{t}$, the roles of $p$ and $q$ can be interchanged, which by this result leads to $\varphi_{F}(q)_{t}>\varphi_{F}(q)_{t}$, a contradiction).

Without loss of generality assume $\varphi_{F}(p)=0 \in A$. Take $r, r^{\prime} \in P^{N}$ such that $r==_{N \backslash\{i\}} r^{\prime}$, best $(r(j))=p(j)$ for all $j \in N$, and best $\left(r^{\prime}(i)\right)=q(i)$. Let $e^{t}$ be the $t$-th unit vector in $\mathbf{R}^{m}$.

Suppose $\varphi_{F}(p)_{t}<\varphi_{F}(q)_{t}$. Let $\lambda \in\left(0, \min \left\{\varphi_{F}(q)_{t}, p(i)_{t}\right\}\right)$, such that $\left[0, \lambda e^{t}\right] \subset A$. Then $F(r) \neq{ }_{\left(0, \mu e^{\prime}\right)} F\left(r^{\prime}\right)$ for all $\mu \in(0, \lambda)$. So by IIA, $r(i) \neq_{\left(0, \mu e^{i}\right)} r^{\prime}(i)$ for all $\mu \in(0, \lambda)$. Furthermore, by (A1) and (A2), $\left(\mu e^{t}, 0\right) \in$ $r(i)$ and $\left(0, \mu e^{t}\right) \notin r(i)$ for all $\mu \in(0, \lambda)$. Hence, $\left(\mu e^{t}, 0\right) \notin r^{\prime}(i)$ or $\left(0, \mu e^{t}\right) \in$ $r^{\prime}(i)$ for all $\mu \in(0, \lambda)$. By (A1) and (A2) this implies that $q(i)_{t} \leq 0$. Consequently, $q(i)_{t}=0$.

Let $s \in A^{N}$ such that $s(i)=\lambda e^{t}$ and $s={ }_{N \backslash\{i\}} p$. Let $\hat{r} \in p^{N}$ with $\hat{r}=N \backslash\{i\} r$ and best $(\hat{r}(i))=s(i)$. Then by (A1) and $(\mathrm{A} 2), \hat{r}(i)=\left(0, \mu e^{i}\right) r(i)$ for all $\mu \in(0, \lambda)$. Hence, by IIA, $F(\hat{r})={ }_{\left(0, \mu e^{e}\right)} F(r)$ for all $\mu \in(0, \lambda)$. So by (A1) and (A2),

$$
\varphi_{F}(s)_{t} \leq 0 \text {. }
$$

Similarly, we have that $\hat{r}(i)={ }_{\left(\mu e^{t}, \lambda e^{t}\right)} r^{\prime}(i)$ for all $\mu \in\left(\lambda, \varphi_{F}(q)_{t}\right)$ with $\mu e^{t} \in A$. So by IIA, $F(\hat{r})={ }_{\left(\mu e^{t}, \lambda e^{t}\right)} F\left(r^{\prime}\right)$ for all $\mu \in\left(\lambda, \varphi_{F}(q)_{t}\right)$ with $\mu e^{t} \in A$. 
By the openness of $A$, and (A1) and (A2) this implies that $\varphi_{F}(s)_{t} \geq \lambda$. This contradicts $(6.1)$. Consequently; $\varphi_{F}(p) \geq \varphi_{F}(q)_{t}$.

Theorem 5.2 states that every uncompromising scheme is a standard coordinatewise veto scheme. So we have:

Corollary 6.1 Let $P, P^{\prime}$ be subclasses of $\hat{P}$ with $P$ full, and let $F: P^{N} \rightarrow P^{\prime}$ satisfy IIA. Then $\varphi_{F}$ is a standard coordinatewise veto scheme.

Lemma 5.2 states that every uncompromising scheme is strategy-proof with respect to $P^{*}$. Since $\hat{P} \subset P^{*}$, we have by corollary 6.1 ,

Corollary 6.2 Let $P, P^{\prime}$ be subclasses of $\hat{P}$ with $P$ full, and let $F: P^{N} \rightarrow P^{\prime}$ satisfy IIA. Then $\varphi_{F}$ is strategy-proof with respect to $P$.

Note the difference with the correspondence theorem of Gibbard and Satterthwaite (Satterthwaite (1975), theorem 2). The class of preferences which they consider is the class of all linear orders over $A$. We will denote this class with $P_{L O}$. It is easy to see that $P_{L O}$ is of totally different structure as $\hat{P}$. None of the two classes is a subclass of the other. The correspondence theorem states that strategy-proofness (with respect to $P_{L O}$ ) of a voting scheme is equivalent to IIA (with respect to $P_{L O}$ ) together with two additional conditions on a corresponding preference scheme. So in this context, strategy-proofness shows to be stronger than IIA. Because of the special structure of $\hat{P}$ (cf. the tops-onlyness condition formulated by lemma 6.2), with respect to (subclasses of) $\hat{P}$ this is the other way around. In section 6.4 we will show that this implies for some subclasses $P$ of $\hat{P}$ that IIA leads to an impossibility result, whereas $S P$ with respect to $P$ gives rise to a whole class of nondictatorial, nonconstant voting schemes.

\subsection{IIA and Euclidean preferences}

In the foregoing section it has been shown that IIA implies uncompromisingness and, thus, strategy-proofness with respect to $\hat{P}$. Further, strategyproofness admits of nonconstant nondictatorial schemes. In this section we demonstrate that IIA is much stronger. Specifically, we prove that restricting attention to only Euclidean preferences leads to an impossibility result, i.e., constantness or dictatorship.

A scheme $\varphi: A^{N} \rightarrow A$ is strongly uncompromising if for all $p, q \in A^{N}$ : $p^{-1}(\{\varphi(p)\}) \subset q^{-1}(\{\varphi(p)\}) \Rightarrow \varphi(p)=\varphi(q)$. So $\varphi$ is strongly uncompromising if changes of individual reports different from the collective optimum, 
do not affect this optimum. Since uncompromisingness guarantees this only for changes into the direction of the compromise point (cf. the definition of uncompromisingness), strong uncompromisingness is indeed more restrictive than uncompromisingness.

It turns out that in case of Euclidean preferences, strong uncompromisingness of a scheme derived from a preference solution is implied by IIA.

Lemma 6.3 Let $A$ be a nonempty open subset of $\mathbf{R}^{m}, m \geq 2$. Let $F$ : $\left(P^{2}\right)^{N} \rightarrow P^{2}$ satisfy IIA. Then $\varphi_{F}$ is strongly uncompromising.

Proof Let $r, r^{\prime} \in\left(P^{2}\right)^{N}$ and $i \in N$ with $r={ }_{N \backslash\{i\}} r^{\prime}$ and $a:=\operatorname{best}(r(i)) \neq$ $\operatorname{best}(F(r))=: y$. Let best $\left(F\left(r^{\prime}\right)\right)=: x$ and best $\left(r^{\prime}(i)\right)=: b$. It is sufficient to prove that $x=y$.

First suppose that $a$ and $b$ are contained in a neighborhood $B$ of $y$ in the open set $A$. Since, by theorem $6.1, \varphi_{F}$ is uncompromising, it is without loss of generality to suppose that $b \neq y$.

Next suppose, additionally, that there exists a hyperplane - hence, several hyperplanes - through $y$ not containing $a$ and $b$ and with $a$ and $b$ on the same side. Let $V$ be such a hyperplane. Take $c, c^{\prime} \in B \backslash V$ with equal (Euclidean) distance to $V$ and on the same straight line perpendicular to $V$. So $c$ is the reflection of $c^{\prime}$ in $V$. Then $r={ }_{\left(c, c^{\prime}\right)} r^{\prime}$, hence $F(r)={ }_{\left(c, c^{\prime}\right)} F\left(r^{\prime}\right)$ by IIA. So, because the Euclidean distance between $y=\operatorname{best}(F(r))$ and $c$ equals the distance between $y$ and $c^{\prime}$ by the choice of $c$ and $c^{\prime}$, the same must be true for $x=\operatorname{best}\left(F\left(r^{\prime}\right)\right)$ instead of $y$. Consequently, $x \in V$. Since this holds for several hyperplanes $V$ it follows that $x=y$.

Next, suppose $a, b, y \in B$ are on the same straight line (so that a hyperplane $V$ as above does not exist). Consider a profile $r^{\prime \prime}$ with $r^{\prime \prime}={ }_{N \backslash\{i\}} r$ and $d:=\operatorname{best}\left(r^{\prime \prime}(i)\right)$ such that $d \in B, d$ is not on the straight line through $a$ and $y$, and $d$ differs from $b$ in only one coordinate, say $s$, with $y_{s}$ not strictly between $b_{s}$ and $d_{s}$. Then, by the argument of the previous paragraph, best $\left(F\left(r^{\prime \prime}\right)\right)=y$. Therefore, by uncompromisingness of $\varphi_{F}, x=$ $\operatorname{best}\left(F\left(r^{\prime}\right)\right)=y$.

Finally, for the general situation with $a$ or $b$ not necessarily in a neighborhood of $y$, choose preferences for individual $i$ with best elements $a^{\prime}$ and $b^{\prime}$, respectively, such that: $a^{\prime}=y$ if $a=y, a^{\prime} \in$ box $(y, a) \backslash\{y\}$ otherwise; $b^{\prime}=y$ if $b=y, b^{\prime} \in \operatorname{box}(y, b) \backslash\{y\}$ otherwise; and $a^{\prime}$ and $b^{\prime}$ are in a neighborhood of $y$. The desired conclusion for the general situation then follows from the cases treated above, and uncompromisingness of $\varphi_{F}$. 
Lemma 6.4 Let $A$ be an open subset of $\mathbf{R}^{m}, m \geq 2$. Let $\varphi: A^{N} \rightarrow A$ be strongly uncompromising. Then $\varphi$ is constant or dictatorial.

Proof Assume $\varphi$ is not constant. We claim that $\varphi(p) \in p(N)$ for every $p \in$ $A^{N}$. Suppose this were not true, i.e., $\varphi(q) \notin p(N)$ for some $q \in A^{N}$. Then, by strong uncompromisingness, $\varphi(p)=\varphi(q)$ for all $p \in A^{N}$, contradicting nonconstantness of $\varphi$.

Next, let $P$ be the subset of $A^{N}$ with $p \in P$ if and only if $|p(N)|=n$. Suppose there are $p, q \in P$ and $i, j \in N$ with $i \neq j, \varphi(p)=p(i)$, and $\varphi(q)=q(j)$. Define $s \in A^{N}$ by $s(i):=p(i), s(j):=q(j), s(l):=p(l)$ otherwise. Then strong uncompromisingness implies $\varphi(p)=\varphi(s)=\varphi(q)$, hence $p(i)=q(j)=: a$. Let $p^{\prime} \in P$ with $\varphi\left(p^{\prime}\right)=p^{\prime}(k)$ for some $k \in N$. Then $k \neq i$ or $k \neq j$. In the former case, it follows in the same way as above that $\varphi\left(p^{\prime}\right)=p^{\prime}(k)=p(i)$. In the latter case, similarly, $\varphi\left(p^{\prime}\right)=p^{\prime}(k)=q(j)$. Therefore, $\varphi\left(p^{\prime}\right)=a$, so $\varphi$ is constant on $P$. This, however, is impossible since $\varphi(p) \in p(N)$ for every $p \in A^{N}$.

Thus, $\varphi$ is dictatorial on $P$. Let individual $\mathrm{i}$ be the dictator. Let $p \in A^{N}$. Take $q \in P$ with $q(i)=p(i)$. By strong uncompromisingness, $\varphi(p)=\varphi(q)=q(i)=p(i)$. Consequently, $\varphi$ is dictatorial on $A^{N}$ (with dictator $i)$. This completes the proof.

Combining lemmas 6.3 and 6.4 we obtain:

Theorem 6.2 Let $A$ be an open subset of $\mathbf{R}^{m}, m \geq 2$. Let $P^{2}$ be the class of Euclidean preferences on $A$, and let $F:\left(P^{2}\right)^{N} \rightarrow P^{2}$ satisfy IIA. Then $\varphi_{F}: A^{N} \rightarrow A$ is constant or dictatorial.

Call a preference scheme constant if it assigns the same preference to every preference profile, and dictatorial if there is an individual whose preference is assigned to any preference profile, then this theorem immediately implies:

Corollary 6.3 Let $A$ be an open subset of $\mathbf{R}^{m}, m \geq 2$. Let $P^{2}$ be the class of Euclidean preferences on $A$, and let $F:\left(P^{2}\right)^{N} \rightarrow P^{2}$ satisfy IIA. Then $F$ is constant or dictatorial.

Theorem 6.2 and corollary 6.3 can be seen as impossibility results: they imply that there are no nonconstant nondictatorial voting schemes under the assumption of IIA with respect to $p^{2}$. These results are similar to Arrow's impossibility theorem (see for instance Muller and Satterthwaite, 1985, theorem 2.2). In this theorem the class of preferences over $A$ may be the class of all weak orders over $A$. On the other hand, $A$ may contain a finite number 
of elements (at least three), whereas in our case, by the openness of $A$, the number of alternatives is infinite. This makes the IIA-condition very restrictive. Since Arrow considers sets which may contain only three alternatives, also the class of preferences he considers may be very small. For this reason he needs some additional requirements to obtain his impossibility result.

In chapter 3 and 4 we characterized classes of voting schemes which were all strategy-proof with respect to $p^{2}$. Many of these classes contained nonconstant nondictatorial schemes. This shows that IIA is a very strong requirement.

\subsection{Conclusion}

We have shown that, in a framework with a continuum of alternatives and restricted domains of preferences, Arrow's independence of irrelevant alternatives condition for preference schemes implies strategy-proofness of the corresponding voting schemes. Furthermore, whereas nonconstant nondictatorial strategy-proof schemes exist, IIA is bound to lead to impossibility results, e.g., if preferences are required to be Euclidean.

We strongly conjecture that similar results can be obtained with respect to classes which are induced by an arbitrary norm on $\mathbf{R}^{m}$ (as discussed in chapter 2). Furthermore, we suspect that in this framework IIA is even stronger than coalitional strategy-proofness (cf. section 2.8, which is on CSP with respect to a strictly convex norm).

Further, it should be noted that $\hat{P}$ is defined with reference to the standard coordinate system (the box $(x, y)$ is defined with reference to this coordinate system). We strongly conject that corollary 6.2 (the implication IIA $\Rightarrow \mathrm{SP}$ ) can be generalized to a preference class $\tilde{P}$, with $\hat{P} \subset \tilde{P}$, whereas $\tilde{P}$ is defined without reference to some coordinate system at all.

So, if we are not misled by our intuitions, many results of this chapter can be generalized in an elegant way.

Our findings till so far correspond to results of Bordes and Le Breton (1988). In a somewhat different setting they too found IIA to be very restrictive. It may be interesting to investigate the connection between their work and ours. 


\section{APPENDIX A}

\section{Metrics and Norms}

Let $X$ be a subset of $\mathbf{R}^{m}$. A metric (or distance function) on $X$ is a function $\delta: X \times X \rightarrow \mathbf{R}$ such that for all $x, y, z \in X$ we have:

(1) $\delta(x, y) \geq 0$

(2) $\delta(x, y)=0$ if and only if $x=y$,

(3) $\delta(x, y)=\delta(y, x)$

(symmetry)

(4) $\delta(x, y) \leq \delta(x, z)+\delta(z, y)$.

(triangular inequality)

$\delta(x, y)$ is called the distance between $x$ and $y$.

In section 2.2 we defined norms on $\mathbf{R}^{\mathbf{m}}$. A norm $\|\cdot\|$ induces a metric $\delta$ by the relation $\delta(x, y) \equiv\|x-y\|$. It is easy to verify that $\delta$ is indeed a metric ( $\delta$ satisfies the properties (1)-(4)).

Norms have some pleasant properties. Some of them are stated in lemma A1, which will be used in chapter 2 of this dissertation. This lemma is closely related to lemma 2.1 , which is on the subclass of strictly convex norms:

Lemma $\mathbf{A 1}$ Let $\|\cdot\|$ be a norm on $\mathbf{R}^{m}$. Then:

(i) For all $x, y, z \in \mathbf{R}^{m}$ such that $\|y\|<\|z\|$ and $x \in[y, z)$ we have that $\|x\|<\|z\|$.

(ii) For all $x, y, z \in \mathbf{R}^{m}$ such that $\|y\| \leq\|z\|$ and $x \in[y, z]$ we have that $\|x\| \leq\|z\|$.

(iii) For all $x, y, z \in \mathbb{R}^{m}$ such that $\|y\|<\|z\|$ and $z \in[y, x)$ we have that $\|z\|<\|x\|$. 
(iv) For all $x, y, z \in \mathbf{R}^{m}$ such that $\|y\| \leq\|z\|$ and $z \in[x, y)$ we have that $\|\boldsymbol{z}\| \leq\|x\|$.

(v) For all $x, y \in \mathbf{R}^{m}$ such that $\|x\|=\|y\|=\frac{1}{2}\|x+y\|$ we have that $x$ and $y$ are $\|\cdot\|$-orthogonal to $x-y$ in the sense of Birkhoff, i.e.

$$
\|x+\lambda(y-x)\| \geq\|x\| \text { for all } \lambda \in \mathbf{R}
$$

and

$$
\|y+\lambda(y-x)\| \geq\|y\| \text { for all } \lambda \in \mathbf{R}
$$

Proof

(i) Let $x, y, z \in \mathbf{R}^{m}$ with $\|y\|<\|z\|$ and $x \in[y, z)$.

Then we can take $\mu \in(0,1]$ with $x=\mu y+(1-\mu) z$. Hence,

$$
\begin{aligned}
\|x\| & =\|\mu y+(1-\mu) z\| \\
& \leq\|\mu y\|+\|(1-\mu) z\| \\
& =\mu\|y\|+(1-\mu)\|z\| \\
& <\|z\| .
\end{aligned}
$$

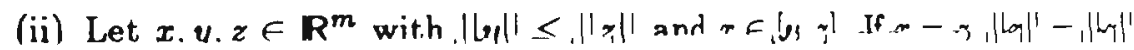
and we are done. If $x \in[y, z)$ the desired result is obtained by taking the proof of (i) and replacing $<$ by $\leq$.

(iii) Let $x, y, z \in \mathbb{R}^{m}$ with $\|y\|<\|z\|$ and $z \in[y, x]$.

If $\|x\| \leq\|y\|$, then by (ii) we have that $\|z\| \leq\|y\|$, which contradicts the assumptions. Hence, $\|y\|<\|x\|$. Consequently, by (i), $\|z\|<\|x\|$.

(iv) Let $x, y, z \in \mathbf{R}^{m}$ with $\|y\| \leq\|z\|$ and $z \in[x, y)$.

If $\|x\|<\|y\|$, then by (i) we have that $\|z\|<\|y\|$, which contradicts the assumptions. Hence $\|y\| \leq\|x\|$. Consequently, by (ii), $\|z\| \leq\|x\|$.

(v) Let $x, y \in \mathbf{R}^{m}$ with $\|x\|=\|y\|=\frac{1}{2}\|x+y\|$.

We will prove that $x$ is $\|\cdot\|$-orthogonal to $y-x$ in the sense of Birkhoff. The proof that $y$ is $\|\cdot\|$-orthogonal to $y-x$ in the sense of Birkhoff is similar. Let $\lambda \in \mathbf{R}$. It suffices to prove that $\|x+\lambda(y-x)\| \geq\|x\|$.

If $\lambda=\frac{1}{2}$ or $x=y$ we are done by assumption. Now assume $\lambda \neq \frac{1}{2}$ and $x \neq y$. If $\lambda>\frac{1}{2}$, then $\frac{1}{2} x+\frac{1}{2} y \in[x, x+\lambda(y-x))$ and hence by (iv), $\|x\|=\left\|\frac{1}{2} x+\frac{1}{2} y\right\| \leq\|x+\lambda(y-x)\|$. 


$$
\begin{aligned}
& \text { If } \lambda<\frac{1}{2}, \text { then } \frac{1}{2} x+\frac{1}{2} y \in[y, x+\lambda(y-x)) \text { and hence by (iv), }\|x\|= \\
& \left\|\frac{1}{2} x+\frac{1}{2} y\right\| \leq\|x+\lambda(y-x)\| .
\end{aligned}
$$

For a more extensive explanation on metrics, norms, inner products and related topics, we refer to Kreyszig (1978). 



\section{References}

Abdou, J., and H. Keiding (1991): Effectivity Functions in Social Choice. Kluwer Academic Publishers, Dordrecht.

Alonso, J., and C. Benítez (1988): "Orthogonality in Normed Linear Spaces: A Survey. Part I: Main Properties", Extracta Mathematica, 3, 1, 1-15.

Arrow, K.J. (1963): Social Choice and Individual Values. Wiley, New York.

Barberà, S., and B. Peleg (1990): "Strategy-Proof Voting Schemes with Continuous Preferences", Social Choice and Welfare, 7, 31-38.

Barberà, S., H. Sonnenschein and L. Zhou (1991): "Voting by Committees", Econometrica, 59, 595-609.

Barberà, S., J. Masso and A. Neme (1992): "Voting under Constraints", Barcelona and San Luis.

Birkhoff, G. (1935): "Orthogonality in linear metric spaces", Duke Math. J., 1, 169-172.

Black, D. (1948): "On the Rationale of Group Decision Making", J. Pol. Econ., 56, 23-24.

Black, D. (1958): The Theory of Committees and Election. Cambridge University Press, Cambridge.

Border, K.C., and J.S. Jordan (1983): "Straightforward Elections, Unanimity and Phantom Voters", Review of Economic Studies, 50, 153-170.

Bordes, G., and M. Le Breton (1989): "Arrovian Theorems with Private Alternatives Domains and Selfish Individuals", Journal of Economic Theory, 47, 257-281. 
Bordes G., G. Laffond and M. le Breton (1990): "Strategy-proofness Issues in Some Economic and Political Domains", Working Paper, GREQE, Marseille.

Chalmet, L. (1983): "Efficiency in Minisum Rectangular Distance Location Problems", Locational Analysis of Public Facilities (ed. by Thisse, Zoller), North-Holland, Amsterdam.

Chichilnisky, G., and G. Heal (1981): "Incentive Compatibility and Local Simplicity", University of Essex and Columbia University.

Chichilnisky, G., and G. Heal (1983): "Necessary and Sufficient Conditions for a Resolution of the Social Choice Paradox", Journal of Economic Theory, 31, 68-87.

Coughlin, P.J. (1990): "Majority Rule and Election Models", Journal of Economic Surveys, 3, 2, 157-188.

Demange, G. (1983): "Spatial Models of Collective Choice", Locational Analysis of Public Facilities (ed. by Thisse, Zoller), North-Holland, Amsterdam.

Gibbard, A. (1973): "Manipulation of Voting Schemes: A General Result", Econometrica, 41, 587-601.

Hurwicz, L., and M. Walker (1990): "On the Generic Non-Optimality of Dominant-Strategy Allocation Mechanisms: A General Theorem that includes Pure Exchange Economies", Econometrica, 58, 683-704.

Kim, K.H., and F.W. Roush (1981): "Properties of Consistent Voting Systems", International Journal of Game Theory, 10, 45-52.

Kim, K.H., and F.W. Roush (1984): "Nonmanipulability in Two Dimensions", Mathematical Social Sciences, 8, 29-43.

Kreyszig, E. (1978): Introductory Functional Analysis With Applications. Wiley, New York.

Laffond, G. (1980): "Révelation des Préférences et Utilités Unimodales", Thesis, Paris.

Le Breton, M., and A. Sen (1991): "Strategyproof Social Choice Functions over Product Domains with Unconditional Preferences", Marseille and New Delhi (second version: 1992). 
Lipschutz, S. (1974): Linear Algebra. McGraw-Hill, New York.

Moreno, D., and M. Walker (1989): "Nonmanipulable Voting Schemes when Participants' Interests are Partially Decomposable", forthcoming in Social Choice and Welfare.

Moreno, D. (1991): "Nonmanipulable Decision Mechanisms For Economic Environments", University of Arizona, Tucson, Arizona.

Moreno, D., and M. Walker (1990): "Nonmanipulable Voting Schemes when Participants' Interests are Partially Decomposable", Report, Department of Economics, University of Arizona, Tucson, Arizona.

Motzkin, T.S. (1935): "Sur quelques propriétés caractéristiques des ensembles convexes", Rend. Reale Acad. Lincei, Classe Sci. Fis., Mat. Nat., Vol. 21, 773-779.

Moulin, H. (1980): "On Strategy-Proofness and Single Peakedness", Public Choice, 35, 437-455.

Moulin, H. (1984): "Generalized Condorcet-Winners for Single Peaked and Single-Plateau Preferences", Social Choice and Welfare, 1, 127-147.

Moulin, H. (1988): Axioms of Cooperative Decision Making. Cambridge University Press, Cambridge.

Muller, E., and M.A. Satterthwaite (1985): "Strategy-proofness: the Existence of Dominant-strategy Mechanisms", Social Goals and Social Organization (ed. by Hurwicz, Schmeidler, Sonnenschein), Cambridge University Press, Cambridge, 131-171.

Nash J.F. (1950): "The bargaining problem", Econometrica, 18, 155-162.

Ostmann, A., and M. Straub (1979): "On the Geometry behind the Fairness Concept of Rawls and Kolm", University of Bielefeld.

Ostmann, A. (1982): "Fair Solutions for Locational Conflicts", Zeitschrift für Operations Research, 26, 87-103.

Peters, H., and H. van der Stel (1990): " A class of Solutions for Multiperson Multicriteria Decision Making", OR Spektrum, 12, 147-153. 
Peters, H., H. van der Stel, and T. Storcken (1991): "On Uncompromisingness and Strategy-proofness", Reports in Operation Research and System Theory, University of Limburg, Mathematics, Maastricht, The Netherlands.

Peters, H., H. van der Stel, and T. Storcken (1992a): "Pareto Optimality, Anonymity, and Strategy-proofness in Location Problems", International Journal of Game Theory, 21, 221-235.

Peters, H., H. van der Stel, and T. Storcken (1992b): "Independence of Irrelevant Alternatives and Strategy-proofness on Economic Domains", in: Game Theory and Economic Applications (ed. by Dutta, Mookherjee, Parthasarathy, Raghavan, Ray, Tijs), Lecture Notes in Economics and Mathematical Systems, 389, Springer Verlag, Berlin.

Peters, H., H. van der Stel, and T. Storcken (1993a): "Generalized Median Solutions, Strategy-Proofness and Strictly Convex Norms", Zeitschrift für Operations Research, 37, forthcoming.

Peters, H., H. van der Stel (1993b): "Range Convexity, Continuity, and Strategy-proofness of Voting Schemes", RM 93-006, Rijksuniversiteit Limburg, Faculteit der Economische Wetenschappen.

Richter, W.F. (1979): "Shapley's Value and Fair Solutions of Location Conflicts", in: Game Theory and Related Topics (ed. by Moeschlin, Pallaschke), North-Holland, Amsterdam.

Rockafellar, R.T. (1970): Convex Analysis. Princeton University Press, Princeton, New Jersey.

Rosenmüller, J. (1980): "Values of non-sidepayment Games and their Application in the Theory of Public Goods", in: Essays in Game Theory and Mathematical Economics, Bibliographisches Institut, Wissenschaftsverlag, Mannheim.

Rosenmüller, J. (1982): "On Values, Location Conflicts, and Public Goods", in: Games, Economic Dynamics, Time Series Analysis. Physica Verlag, Wien-Würzburg.

Rubinstein, A., and P.C. Fishburn (1986): "Algebraic Aggregation Theory", Journal of Economic Theory, 38, 63-77. 
Satterthwaite, M. (1975): "Strategy-Proofness and Arrow's Conditions: Existence and Correspondence Theorem for Voting Procedures and Social Choice Functions", Journal of Economic Theory, 10, 187-217.

Satterthwaite, M., and H. Sonnenschein (1981): "Strategy-Proof Allocation Mechanisms at Differentiable Points", Review of Economic Studies, $48,587-597$.

Small, G.C. (1990): "A Survey of Multidimensional Medians", International Statistical Review, 58, 3, 263-277.

Sprumont, Y. (1991): "The Division Problem with Single-Peaked Preferences: A Characterization of the Uniform Allocation Rule", Econometrica, 59, 509-519.

Valentine (1964): Convex Sets. McGraw-Hill, New York.

Ward, J.E., and R.E. Wendell (1983): "Characterizing Efficient Points in Location Problems under the One-infinity Norm", Locational Analysis of Public Facilities (ed. by Thisse, Zoller), North-Holland, Amsterdam.

Zhou, L. (1991): "Impossibility of Strategy-Proof Mechanisms in Economies with Pure Public Goods", Review of Economic Studies, 58, 107-119. 



\section{List of Symbols}

Many symbols are defined in section 1.2. Here we list some notations which are defined elsewhere, and where these definitions can be found:

$\begin{array}{ll}\varphi_{\gamma} & 21,125 \\ \|\cdot\|_{1} & 25 \\ \|\cdot\|_{p} & 26 \\ \|\cdot\|_{\infty} & 27 \\ & \\ \langle\cdot \cdot \cdot\rangle & 92 \\ \rho^{*} & 123 \\ \rho^{p} & 124 \\ \rho^{\infty} & 124 \\ \rho_{\alpha} & 133 \\ Q^{k} & 141 \\ \hat{p} & 148\end{array}$

$\delta(x, y) \quad$ Appendix A 



\section{Subject Index}

$\begin{array}{ll}\|\cdot\| \text {-orthogonal } & 29 \\ \|\cdot\| \text {-orthogonal in the sense of Birkhoff } & 28 \\ \text { agents } & 7 \\ \text { anonymity } & 11 \\ \text { assignment of disagreements } & 20,125 \\ \text { best } & 8 \\ \text { Birkhoff } & 28 \\ \text { bliss point } & 8 \\ \text { box } & 123 \\ \text { citizen's sovereignty } & 10 \\ \text { coalitional strategy-proofness } & 10,42 \\ \text { compromise point } & 7 \\ \text { confirmatoriness } & 15 \\ \text { constant } & 8,153 \\ \text { coordinatewise median scheme } & 18 \\ \text { coordinatewise veto scheme } & 21 \\ \text { coordinatewise midpoint closed } & 142 \\ \text { core } & 126 \\ \text { dictator } & 8,129,153 \\ \text { dictatorial scheme } & 8 \\ \text { effectivity function } & 125 \\ \text { efficiency } & 10 \\ \text { Euclidean metric } & 93 \\ \text { Euclidean norm } & 93 \\ \text { full } & 149 \\ \text { ideal point } & 8 \\ \text { impossibility result } & 8 \\ \text { independence of irrelevant alternatives } & 149 \\ \text { individuals } & 7 \\ & \\ & \end{array}$




\begin{tabular}{|c|c|}
\hline induced by & 25,41 \\
\hline inner product & 92 \\
\hline intermediate strategy-proofness & 10,42 \\
\hline location problem & 1 \\
\hline majority rule & 12 \\
\hline metric & 25 ,appendix A \\
\hline nondictatorial & 11 \\
\hline norm & 25 \\
\hline option set & 110 \\
\hline orthogonal & 28 \\
\hline Pareto optimality & 9,42 \\
\hline phantom voters & 18 \\
\hline positive association & 50 \\
\hline preference & 7 \\
\hline preference scheme & 149 \\
\hline preference profile & 149 \\
\hline prescheme & 7 \\
\hline profile & 7,149 \\
\hline range convexity & 9 \\
\hline scherne & 7 \\
\hline separable & 126 \\
\hline standard coordinatewise veto scheme & 21,125 \\
\hline standard & 14 \\
\hline standard inner product & 93 \\
\hline strategy-proofness & 10,42 \\
\hline strict strategy-proofness & 11 \\
\hline strictly convex norms & 27 \\
\hline strong uncompromisingness & 151 \\
\hline strong Pareto optimality & 9,42 \\
\hline surjectivity & 9 \\
\hline tops-onlyness & 150 \\
\hline transitive & 12 \\
\hline translation covariant & 74 \\
\hline unanimity & 9 \\
\hline uncompromisingness & 124,151 \\
\hline unit ball & 26 \\
\hline voters & 7 \\
\hline voting scheme & 7 \\
\hline weak Pareto optimality & 9,42 \\
\hline
\end{tabular}




\section{Abbreviations}

$\begin{array}{ll}\text { AN } & \text { anonymity } \\ \text { CSP } & \text { coalitional strategy-proofness } \\ \text { ISP } & \text { intermediate strategy-proofness } \\ \text { ND } & \text { nondictatoriness } \\ \text { PO } & \text { Pareto optimality } \\ \text { RC } & \text { range convexity } \\ \text { SP } & \text { strategy-proofness } \\ \text { SPO } & \text { Strong Pareto optimality } \\ \text { SSP } & \text { strict strategy-proofness } \\ \text { SURJ } & \text { surjectivity } \\ \text { UN } & \text { unanimity } \\ \text { w.l.o.g. } & \text { without loss of generality } \\ \text { WPO } & \text { Weak Pareto optimality }\end{array}$





\section{Author Index}

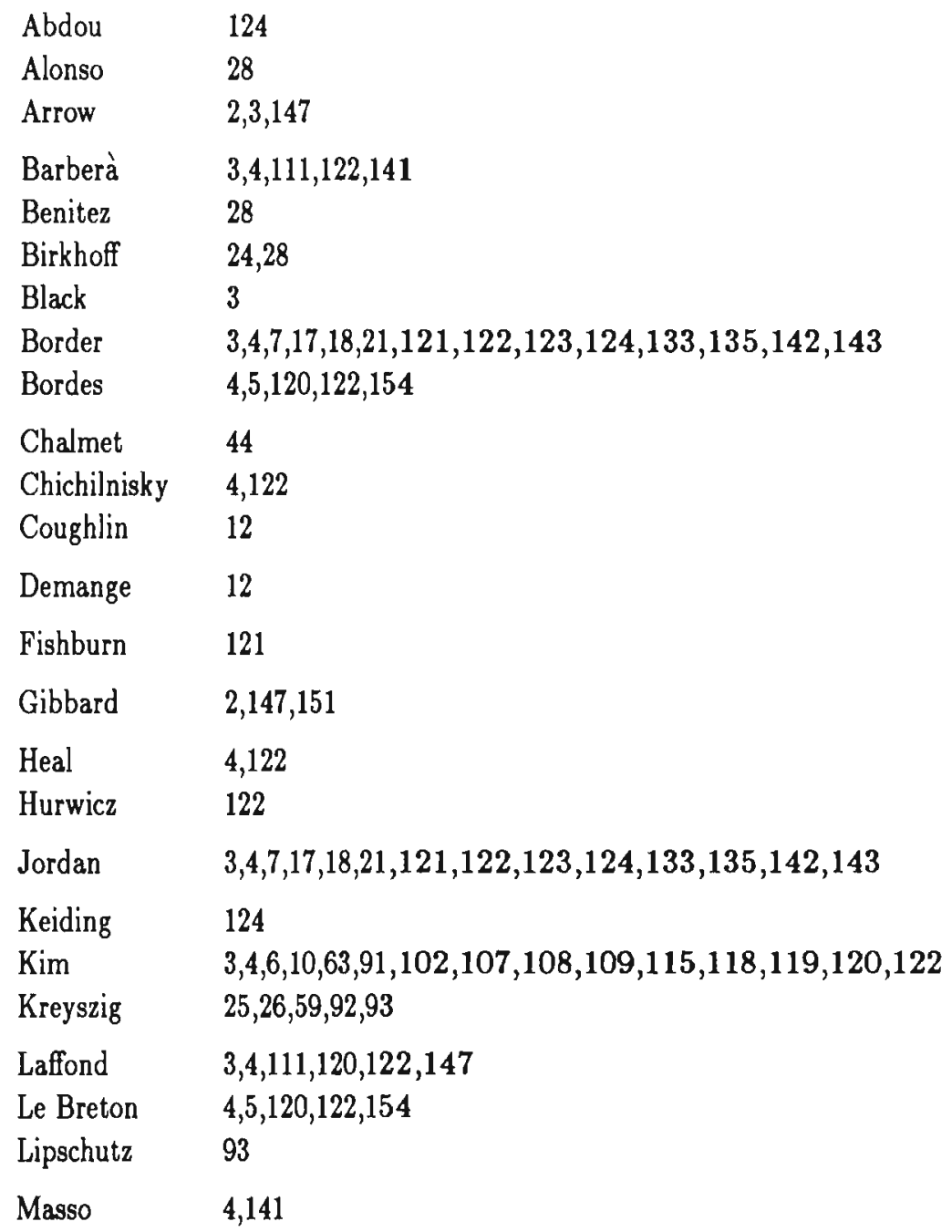




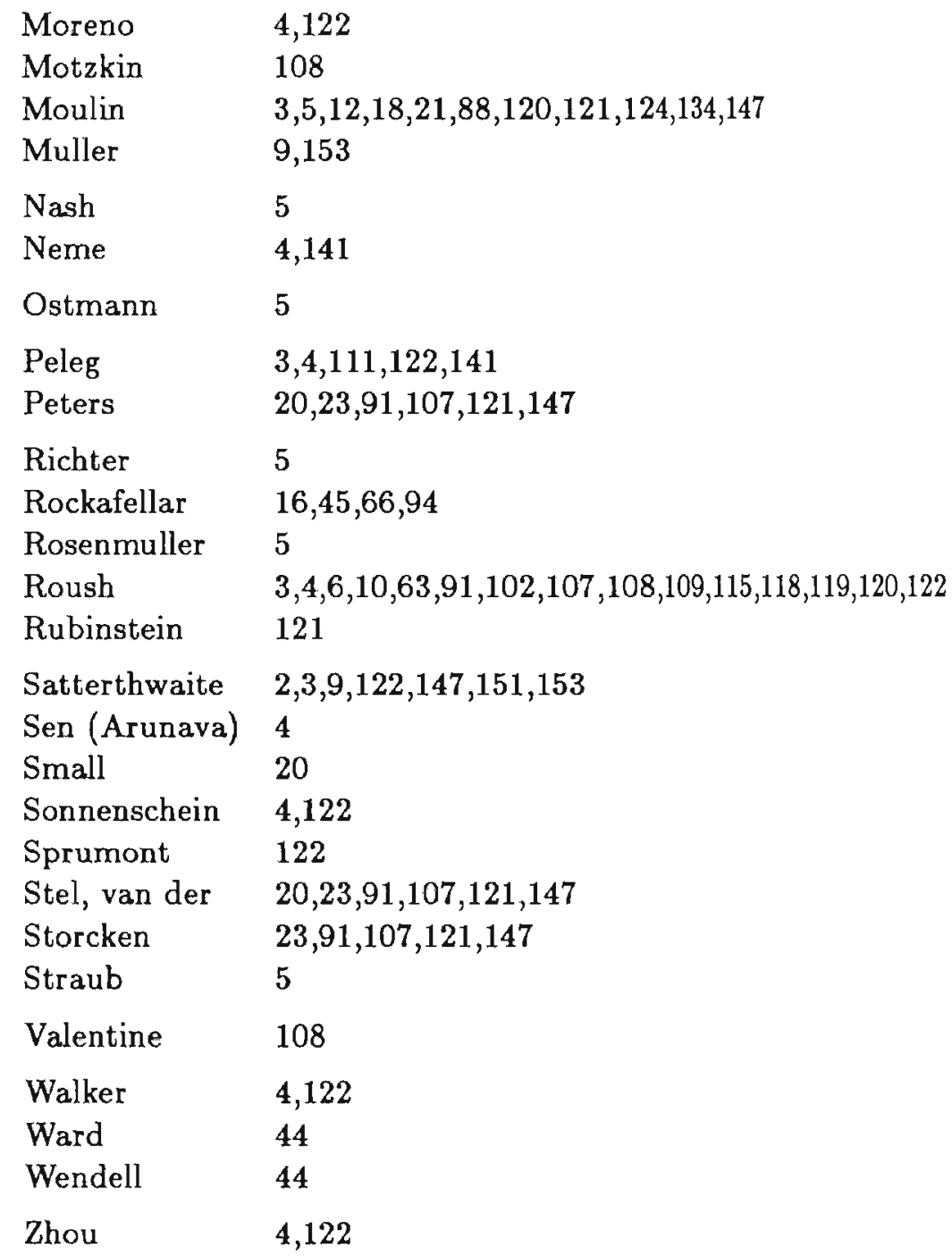




\section{Samenvatting}

In dit proefschrift bestuderen wij beslissingsproblemen waarbij een groep individuen overeenstemming moet zien te bereiken omtrent de keuze van één alternatief uit een gegeven alternatievenverzameling. Een simpele en directe manier om tot een beslissing te komen is de volgende procedure: ieder individu noemt één alternatief. Vervolgens wordt er een regel toegepast die op basis van de genoemde alternatieven de uiteindelijke uitkomst bepaalt. Een dergelijke regel zullen we een stemregel noemen.

De individuen worden in hun stemgedrag geleid door hun preferenties over de alternatievenverzameling. We gaan er van uit, dat deze preferenties zó zijn, dat er voor elk individu één of meerdere alternatieven bestaan die zijn voorkeur genieten boven alle andere alternatieven.

Omtrent de alternatieven veronderstellen we dat deze gerepresenteerd kunnen worden door punten in een $m$-dimensionale Euclidische ruimte. De alternatievenverzameling waar we vanuit gaan, zal doorgaans bestaan uit een continuum. Het resulterende beslissingsprobleem duiden we aan als een $m$-dimensionaal $n$-persoons lokatieprobleem.

Zo'n probleem kan een lokatieprobleem in de letterlijke zin des woords zijn. Het kan echter ook een meer abstract beslissingsprobleem zijn, waarbij een groep personen een $m$-tal kwantiteiten moet vaststellen. Dit kunnen geldbudgetten zijn, gewichten voor multi-criteria analyse, of bijvoorbeeld beslissingsvariabelen ten behoeve van één of ander beleidsproces, etc.

We gaan er van uit dat de voorkeuren die iemand in werkelijkheid heeft, uiteindelijk slechts de persoon in kwestie bekend zijn. Dit houdt in dat iemand de vrijheid heeft om in zijn stemgedrag af te wijken van zijn werkelijke voorkeuren. Hij zal dat waarschijnlijk niet nalaten, als hij hiermee het beslissingsproces in zijn voordeel kan beïnvloeden. In dit proefschrift concentreren wij ons op stemregels die niet-manipuleerbaar zijn. Dit betekent dat een stemregel zodanig is, dat een individu er niets mee kan winnen door af te wijken van zijn werkelijke voorkeuren. Indien een stemregel niet- 
manipuleerbaar is heeft dit belangrijke voordelen:

(i) Om tot een 'goede' uitkomst van het beslissingsproces te komen, is het noodzakelijk om over betrouwbare informatie over de individuele voorkeuren te beschikken.

(ii) Het is overbodig om zich druk te maken over de strategische keuze van het alternatief dat men zal rapporteren. Het volstaat om één der favoriete alternatieven te noemen.

(iii) Individuen die een favoriet alternatief noemen, worden niet benadeeld ten opzichte van anderen (als ze wèl benadeeld worden, is dit op zichzelf al een motief om af te wijken).

In de sociale keuzetheorie zijn enkele onmogelijkheidsstellingen bekend met betrekking tot het bestaan van redelijke niet-manipuleerbare stemregels. Deze onmogelijkheidsresultaten pareren we door angaande de individuele preferenties enkele veronderstellingen te maken. Hierdoor perken we de klasse van mogelijke preferenties in, waardoor de niet-manipuleerbaarheidsconditie zwakker wordt.

In de hoofdstukken 2, 3 en 4 nemen we aan dat alle preferenties door dezelfde metriek op $\mathbf{R}^{m}$ worden geïnduceerd. We noemen een preferentie geinduceerd door de metriek $\delta$ op $\mathbf{R}^{m}$, indien er een punt $z$ in $\mathbf{R}^{m}$ bestaat zodat een punt hoger gewaardeerd wordt naarmate het dichter ligt bij $z$, gemeten volgens de metriek $\delta$.

In hoofdstuk 2 gaan we er van uit dat $\delta$ geinduceerd wordt door een strikt convexe norm. Voor $m=2$ karakteriseren we de verzameling van stemregels die niet-manipuleerbaar, zwak Pareto optimaal en anoniem zijn. Een stemregel noemen we zwak Pareto optimaal, als deze voor elke combinatie van gerapporteerde punten een compromispunt voorschrijft zodanig, dat er geen ander punt in de alternatievenverzameling bestaat dat voor alle individuen aantrekkelijker is dan het voorgeschreven punt. Een stemregel noemen we anoniem, als deze geen onderscheid maakt tussen de individuen. Tevens bewijzen we in hoofdstuk 2 de existentie van de klasse van stemregels die we gekarakteriseerd hebben. De bewijsstructuur van dit hoofdstuk is gecompliceerd, en omvat onder andere de ontwikkeling van enige theorie op het gebied van orthogonaliteit in eindig-dimensionale vectorruimten.

In hoofdstuk 3 gaan we uit van de Euclidische metriek. Wederom karakteriseren we voor $m=2$ de klasse van niet-manipuleerbare, zwak Pareto optimale, anonieme stemregels. De structuur van het bewijs van dit resultaat verschilt essentieel van die in het voorgaande hoofdstuk. Het bewijs is 
ook veel korter. Dat komt, omdat we in dit geval gebruik kunnen maken van een stelling van Kim en Roush (1984). Verder laten we in dit hoofdstuk zien dat het resultaat ook geldig is voor willekeurige door een inwendig produkt geinduceerde metrieken. Tevens tonen we aan dat er voor $m-2$ geen stemregels zijn die aan de gestelde voorwaarden voldoen.

In hoofdstuk 4 gaan we wederom uit van de Euclidische metriek. We karakteriseren de klasse van stemregels die niet-manipuleerbaar en anoniem zijn, en die tevens een convex bereik hebben. Hierbij maken we nogmaals gebruik van het resultaat van Kim en Roush (1984).

In hoofdstuk 5 bestuderen we een geheel andere klasse van preferenties, de klasse $P^{*}$. Kort samengevat is $P^{*}$ de klasse van preferenties die minimaal één ideaal punt hebben en die zodanig zijn dat punten niet aantrekkelijker worden naarmate men coördinaatsgewijze van een ideaal punt vandaan loopt. Als alternatievenverzameling nemen we een open deelverzameling van $\mathbf{R}^{m}$. Aangezien de klasse $P^{*}$ veel preferenties bevat, is de nietmanipuleerbaarheidsconditie die we hier hanteren veel sterker dan die in de voorgaande hoofdstukken. Dit stelt ons in staat om de klasse van alle niet-manipuleerbare stemregels te karakteriseren.

In hoofdstuk 6 gaan we uit van een deelklasse van $P^{*}$. We richten onze aandacht in eerste instantie op zogeheten preferentieregels. Dit zijn afbeeldingen die individuele preferenties aggregeren tot collectieve preferenties. We concentreren ons op preferentieregels die aan Arrow's (1963) IIA-conditie (onafhankelijkheid van irrelevante alternatieven) voldoen. Deze conditie houdt in dat de collectieve preferentie tussen twee alternatieven bepaald wordt door de individuele preferenties tussen deze alternatieven. We gaan nu uit van een willekeurige deelklasse $P$ van $P^{*}$ die dusdanig is dat elk alternatief een ideaal punt is van een preferentie binnen $P$. We tonen aan dat met elke preferentieregel die aan de IIA-conditie voldoet een nietmanipuleerbare stemregel geassocieerd kan worden. Tevens tonen we aan dat IIA met betrekking tot de klasse van Euclidische preferenties (die we in hoofdstukken 3 en 4 bestudeerd hebben) tot een onmogelijkheidsresultaat leidt. Dit duidt erop, dat IIA in de context van dit proefschrift een veel zwaardere eis is dan niet-manipuleerbaarheid. 


\section{Curriculum vitae}

Hans van der Stel werd geboren op 28 mei 1960 te Goes. Van 1972 tot 1978 volgde hij voorbereidend wetenschappelijk onderwijs aan de Roncalli Scholengemeenschap te Bergen op Zoom en de Christelijke Scholengemeenschap "Walcheren" te Middelburg. $\mathrm{Na}$ het behalen van zijn eindexamen studeerde hij vanaf 1978 Toegepaste Wiskunde aan de Technische Universiteit Delft. In het kader van een afstudeerstage bij de Deltadienst van Rijkswaterstaat schreef hij een doctoraalscriptie over de incorporatie van buoyancy in een algengroeimodel.

Hij studeerde in 1985 af bij Prof. dr. M.S. Keane met het vak Operationele Analyse als specialisatie.

In 1986 vervulde hij zijn militaire dienstplicht. In 1986 was hij als assistent logistiek manager werkzaam bij PTT Telecom. Per 1 februari 1988 trad hij in dienst bij de vakgroep Wiskunde van de Rijksuniversiteit Limburg. De belangrijkste resultaten van het daar verrichte onderzoek zijn weergegeven in dit proefschrift. 

